\author{
Supporting Information \\ (CWiley-VCH 2019 \\ 69451 Weinheim, Germany
}

\title{
A Chiral Naphthyridine Diimine Ligand Platform Enables Nickel-Catalyzed Asymmetric Alkylidenecyclopropanations
}

Elena Braconi and Nicolai Cramer*

\author{
EPFL SB ISIC LCSA, BCH 4305 \\ 1015 Lausanne (Switzerland) \\ E-mail: nicolai.cramer@epfl.ch \\ Homepage: http://isic.epfl.ch/lcsa
}

\begin{abstract}
A novel class of chiral naphthyridine diimine ligands $\left(\mathrm{NDI}^{*}\right)$ readily accessible from $C_{2}$-symmetric 2,6 -di-(1-arylethyl)anilines is described. Their utility, in particular a member with fluorinated aryl side arms, is demonstrated by a reductive Ni-catalyzed enantioselective alkylidene transfer reaction from 1,1-dichloroalkenes to olefins. This transformation provides direct access to a broad range of synthetically valuable alkylidenecyclopropanes in high yields and enantioselectivities.
\end{abstract}

DOI: 10.1002/anie.2016XXXXX 


\section{Table of Contents}

Table of Contents 2

General methods 3

Experimental Procedures $\quad 4$

1. Synthesis and characterization of 1,1'-(1,8-Naphthyridine-2,7-diyl)diethanone 2

2. Synthesis and characterization of chiral anilines $1 \mathbf{a}-\mathbf{i}$

3. Synthesis and characterization of chiral ligands L1-9 24

4. Synthesis and characterization of novel 1,1-dichloroalkenes 34

5. General procedure for racemic alkylidene transfer 35

6. Optimization of the enantioselective alkylidene transfer 36

7. General procedure for enantioselective alkylidene transfer (GP3) 37

8. Characterization data of chiral alkylidenecyclopropanes 6

- Scale-up reaction (1.00 mmol scale) 50

9. Derivatization of chiral alkylidenecyclopropanes 85

- Absolute configuration determination of 7

X-ray crystallographic data 97

X-ray crystallographic data for L1 97

X-ray crystallographic data for L9 99

${ }^{1} \mathrm{H},{ }^{13} \mathrm{C}$ and ${ }^{19} \mathrm{~F}$ NMR Spectra 101

References 163 


\section{General methods}

All reactions were carried out under an atmosphere of nitrogen in oven-dried glassware with magnetic stirring, unless otherwise indicated. THF, $\mathrm{CH}_{2} \mathrm{Cl}_{2}, \mathrm{MeOH}$ and toluene were purified by an Innovative Technology Solvent Delivery System. Dry $\mathrm{Et}_{2} \mathrm{O}$ and CPME were degassed by freeze-pump-thaw $(3 \times)$ before being stored in the glovebox under $4 \AA$ molecular sieves. All other solvents were used as purchased. Chemicals were used as obtained from the suppliers. Flash chromatography was performed with Silicycle silica gel 60 (0.040-0.063 $\mu \mathrm{m}$ grade) or neutral alumina (Aluminium oxide neutral, Brockmann I). Analytical thin-layer chromatography was performed with commercial glass plates coated with $0.25 \mathrm{~mm}$ silica gel (E. Merck, Kieselgel 60 F254). Compounds were either visualised under UV-light at $254 \mathrm{~nm}$ or by dipping the plates in an aqueous potassium permanganate solution followed by heating. Proton nuclear magnetic resonance $\left({ }^{1} \mathrm{H} \mathrm{NMR}\right)$ data were acquired on a Bruker $A V 400(400 \mathrm{MHz})$. Chemical shifts $(\delta)$ are reported in parts per million (ppm) relative to incompletely deuterated $\mathrm{CDCl}_{3}$ (s, 7.26 ppm). Splitting patterns are designated as s, singlet; d, doublet; t, triplet; q, quartet; p, pentet; dd, doublet of doublets; td, triplet of doublets; ddd, doublet of doublet of doublet; m, multiplet; br, broad. Proton decoupled Carbon13 nuclear magnetic resonance $\left({ }^{13} \mathrm{C} \mathrm{NMR}\right)$ data were acquired at on a Bruker $A V 400(101 \mathrm{MHz})$. Chemical shifts are reported in ppm relative to $\mathrm{CDCl}_{3}(77.16 \mathrm{ppm})$. Proton decoupled Flourine-19 nuclear magnetic resonance $\left({ }^{19} \mathrm{~F}\right.$ NMR) were acquired at $376 \mathrm{MHz}$ on a Bruker AV400 spectrometer. Infrared (IR) data were recorded on an Alpha-P Bruker FT-IR Spectrometer. Absorbance frequencies are reported in reciprocal centimeters $\left(\mathrm{cm}^{-1}\right)$. HRMS measurements were performed by an Agilent LC-MS TOF or Orbitrap. High resolution mass are given in $\mathrm{m} / \mathrm{z}$. Enantiomeric excesses were measured on an Agilent Normal or Reverse Phase HPLC, or on a Thar SFC Investigator system using chiral stationary phase columns. Optical rotations were measured on a Polartronic M polarimeter using a $0.5 \mathrm{~cm}$ cell with a $\mathrm{Na}$ $589 \mathrm{~nm}$ filter. Melting points were measured on a Büchi melting point apparatus, model B-540, and are uncorrected. X-ray analysis was performed by Dr. R. Scopelliti and Dr. F. Fadaei Tirani at the EPF Lausanne. 


\section{SUPPORTING INFORMATION}

\section{Experimental Procedures}

\section{Synthesis and characterization of 1,1'-(1,8-Naphthyridine-}

\section{2,7-diyl)diethanone 2}

1,1'-(1,8-Naphthyridine-2,7-diyl)diethanone 2 was prepared according to the following synthetic procedure. (Scheme 1)

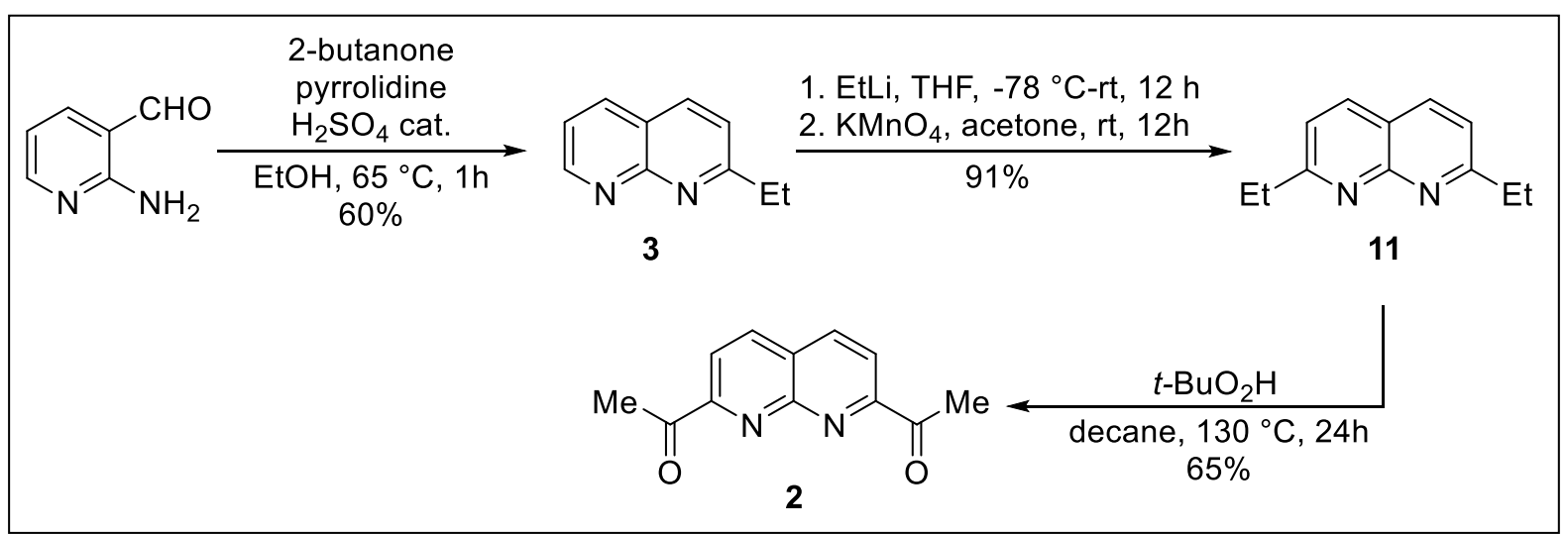

Scheme 1: Synthetic procedure to access 1,1'-(1,8-Naphthyridine-2,7-diyl)diethanone 2.

\section{2-Ethyl-1,8-naphthyridine 3}

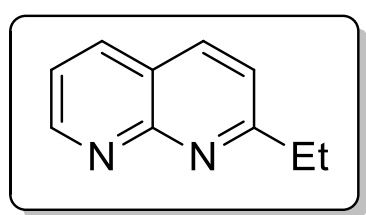

Prepared according to a reported procedure, spectroscopic data matched those previously reported in the literature. ${ }^{1}$

Yield: $60 \%$

${ }^{1}$ H NMR (400 MHz, Chloroform-d): $\delta 9.08(\mathrm{dd}, J=4.3,2.0 \mathrm{~Hz}, 1 \mathrm{H}), 8.19-8.06(\mathrm{~m}, 2 \mathrm{H}), 7.48-7.37$ $(\mathrm{m}, 2 \mathrm{H}), 3.09(\mathrm{q}, J=7.6 \mathrm{~Hz}, 2 \mathrm{H}), 1.45(\mathrm{t}, J=7.6 \mathrm{~Hz}, 3 \mathrm{H})$.

${ }^{13}$ C NMR (101 MHz, Chloroform-d): $\delta$ 167.98, 156.15, 153.38, 137.09, 136.81, 122.27, 121.50, 121.14, $32.51,13.49$. 


\section{2,7-Diethyl-1,8-naphthyridine 11}

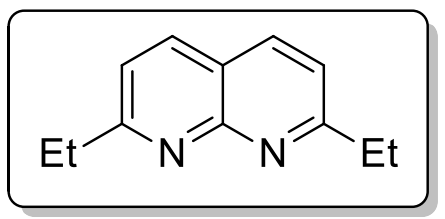

Adapted from a literature procedure. ${ }^{2}$ To a flame dried $250 \mathrm{~mL}$ two-neck round bottom flask equipped with a dropping funnel and under $\mathrm{N}_{2}$ atmosphere, were added 2-ethyl-1,8-naphthyridine 3 (3.00 g, 19.0 mmol, 1.00 equiv.) and dry THF $(76.0 \mathrm{~mL}, 0.25 \mathrm{M})$. The yellow solution was cooled to $-78^{\circ} \mathrm{C}$, whereby ethyllithium (33.2 mmol, 1.75 equiv., $0.50 \mathrm{M}$ solution in benzene/cyclohexane) was added dropwise at this temperature. The corresponding brown solution was allowed to warm to room temperature and stirred until TLC showed disappearance of starting material (approximately 16 hours). The reaction mixture was cooled to $0{ }^{\circ} \mathrm{C}$ and quenched with $\mathrm{H}_{2} \mathrm{O}(20.0 \mathrm{~mL}), \mathrm{Et}_{2} \mathrm{O}$ was added, layers were separated, the aqueous layer was extracted with $\mathrm{Et}_{2} \mathrm{O}(2 \times)$. The combined organics were dried over $\mathrm{MgSO}_{4}$, filtered, and concentrated under reduced pressure to afford the intermediate 2,7-diethyl-1,2-dihydro-1,8-naphthyridine as an orange oil. A saturated solution of $\mathrm{KMnO}_{4}$ in acetone $(400 \mathrm{~mL})$ was added to the intermediate and the mixture was left stirring for 16 hours, whereby it was filtered over a plug of silica and concentrated under reduced pressure to afford 2,7-diethyl-1,8-naphthyridine 11. The product was used in the next step without further purification.

Yield: $91 \%$

Appearance: off-white solid

${ }^{1}$ H NMR (400 MHz, Chloroform-d): $\delta 8.05(\mathrm{~d}, J=8.3 \mathrm{~Hz}, 2 \mathrm{H}), 7.34(\mathrm{~d}, J=8.2 \mathrm{~Hz}, 2 \mathrm{H}), 3.07$ (q, $J=$ $7.6 \mathrm{~Hz}, 4 \mathrm{H}), 1.43$ (t, $J=7.6 \mathrm{~Hz}, 6 \mathrm{H})$.

${ }^{13}$ C NMR (101 MHz, Chloroform-d): $\delta$ 167.66, 155.87, 136.78, 121.25, 119.23, 32.53, 13.78 .

IR (ATR): $v\left(\mathrm{~cm}^{-1}\right)$ 3006, 2975, 2934, 2873, 1601, 1539, 1506, 1452, 1436, 1414, 1304, 1141, 1054, 859, 822.

HRMS (ESI/QTOF): calcd for $\mathrm{C}_{12} \mathrm{H}_{15} \mathrm{~N}_{2}{ }^{+}[\mathrm{M}+\mathrm{H}]^{+}$: 187.1230; found: 187.1235.

Melting point: $101.8-103.2{ }^{\circ} \mathrm{C}$

\section{$\underline{1,1^{\prime}-(1,8-N a p h t h y r i d i n e-2,7-d i v l) \text { diethanone } 2}$}

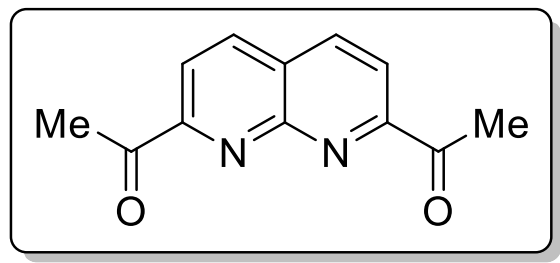


Adapted from a literature procedure. ${ }^{3}$ To a flame dried Ace pressure tube were added 2,7-diethyl-1,8naphthyridine 9 ( $1.50 \mathrm{~g}, 8.05 \mathrm{mmol}, 1.00$ equiv.) and tert-butyl hydroperoxide (193 mmol, 24.0 equiv., $5.50 \mathrm{M}$ solution in decane). The tube was sealed and the corresponding yellow solution was heated at $130{ }^{\circ} \mathrm{C}$ until TLC showed disappearance of starting material (approximately 24 hours). The reaction mixture was allowed to cool to room temperature; $\mathrm{H}_{2} \mathrm{O}(50 \mathrm{~mL})$ and EtOAc $(50 \mathrm{~mL})$ were added, layers were separated, the aqueous layer was extracted with EtOAc $(2 x)$. The combined organics were dried over $\mathrm{MgSO}_{4}$, filtered, and concentrated under reduced pressure. Purification via flash column chromatogtraphy ( $\mathrm{SiO}_{2}, 20 \%$ EtOAc in pentane) afforded the title compound 2. Spectroscopic data matched those previously reported in the literature. ${ }^{4}$

Yield: $65 \%$

${ }^{1} \mathrm{H}$ NMR (400 MHz, Chloroform-d): $\delta 8.41(\mathrm{~d}, J=8.5 \mathrm{~Hz}, 2 \mathrm{H}), 8.32(\mathrm{~d}, J=8.4 \mathrm{~Hz}, 2 \mathrm{H}), 2.98(\mathrm{~s}, 6 \mathrm{H})$. ${ }^{13}$ C NMR (101 MHz, Chloroform- $d$ ): $\delta$ 200.16, 156.92, 154.50, 138.48, 126.45, 120.82, 26.11. 


\section{Synthesis and characterization of chiral anilines 1a-i}

Anilines 12a-i were synthesized via either Route $\boldsymbol{A}$ or Route $\boldsymbol{B}$ according to a previously reported procedure, ${ }^{5}$ which is summarized in Scheme 2A. Subsequent asymmetric hydrogenation ( $\underline{\text { GP1 }}$ ) afforded chiral anilines 1a-i. (Scheme 2B)

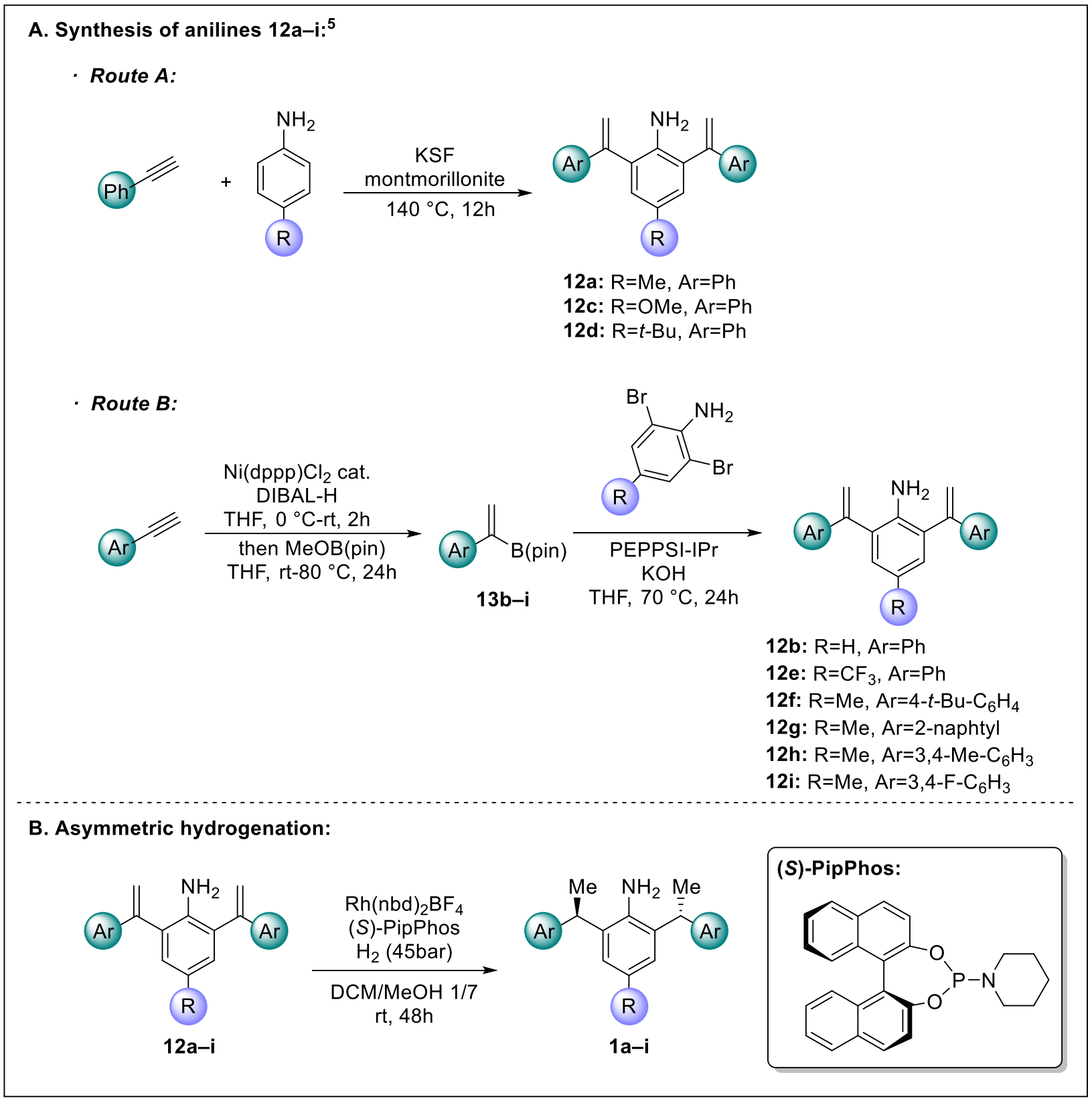

Scheme 2: Synthetic procedure to access chiral anilines 1a-i.

\section{General procedure for asymmetric hydrogenation (GP1)}

In a flame dried microwave tube and under a $\mathrm{N}_{2}$ atmosphere, $\left[\mathrm{Rh}(\mathrm{nbd})_{2}\right] \mathrm{BF}_{4}(2.00 \mathrm{~mol} \%)$ and $1-((11 \mathrm{bS})-$ dinaphtho[2,1-d:1',2'-f][1,3,2]dioxaphosphepin-4-yl)piperidine PipPhos (4.40 mol\%) were dissolved in 
$\operatorname{DCM}(0.50 \mathrm{ml})$ and stirred for $20 \mathrm{~min}$. Subsequently a solution of alkenyl aniline 12 (1.00 equiv.) in a minimal amount of DCM was added. $\mathrm{MeOH}$ (ratio MeOH/DCM: >7/1, 0.20M) was added. The microwave tube was transferred into an autoclave and the reactor was purged three times with $\mathrm{H}_{2}$. The reactor was then pressurized to 45 bar with $\mathrm{H}_{2}$ and the mixture was stirred at ambient temperature for $48 \mathrm{~h}$. The reaction mixture was concentrated under reduced pressure and purified by silica gel column chromatography to yield chiral anilines $\mathbf{1 a}-\mathbf{i}$.

\section{$\underline{\text { 2-(1-(3,5-Difluorophenyl)vinyl)-4,4,5,5-tetramethyl-1,3,2-dioxaborolane 13i }}$}

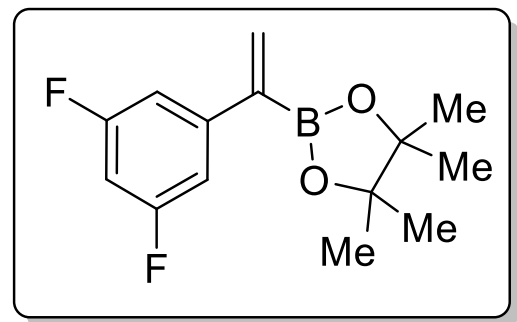

Yield: $80 \%$

Appearance: colourless oil

${ }^{1}$ H NMR (400 MHz, Chloroform-d): $\delta 7.09-6.98$ (m, 2H), $6.68(\mathrm{tt}, J=8.9,2.3 \mathrm{~Hz}, 1 \mathrm{H}), 6.17-6.08$ (m, 2H), 1.33 (s, 12H).

${ }^{13}$ C NMR (101 MHz, Chloroform-d): $\delta 162.99$ (dd, $\left.J=248,14.1 \mathrm{~Hz}\right), 144.72(\mathrm{t}, J=9.09 \mathrm{~Hz}), 132.97$, $110.165(\mathrm{dd}, J=18.6,7.07 \mathrm{~Hz}), 102.36(\mathrm{t}, J=25.7 \mathrm{~Hz}), 84.25,24.93$.

${ }^{19}$ F NMR (376 MHz, Chloroform-d): $\delta-111.00$.

IR (ATR): $v\left(\mathrm{~cm}^{-1}\right)$ 2980, 2932, 1621, 1598, 1412, 1377, 1319, 1294, 1143, 1117, 987, 952, 863, 838.

HRMS (APPI/LTQ-Orbitrap): calcd for $\mathrm{C}_{14} \mathrm{H}_{17} \mathrm{BF}_{2} \mathrm{O}_{2}{ }^{+}[\mathrm{M}]^{+}:$266.1284; found 266.1281.

\section{2,6-Bis(1-phenylvinyl)aniline 12b}

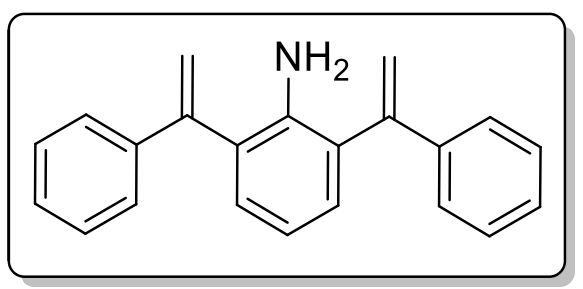

Yield: 99\% (Route B)

Appearance: yellow oil

${ }^{1}$ H NMR (400 MHz, Chloroform-d): $\delta 7.40$ (m, 4H), $7.35-7.27$ (m, 6H), 7.15 (dd, J = 7.6, 2.0 Hz, 2H), $6.83(\mathrm{td}, J=7.6,1.8 \mathrm{~Hz}, 1 \mathrm{H}), 5.83(\mathrm{~d}, J=1.6 \mathrm{~Hz}, 2 \mathrm{H}), 5.41(\mathrm{~d}, J=1.6 \mathrm{~Hz}, 2 \mathrm{H}), 3.54(\mathrm{bs}, 2 \mathrm{H})$. 
${ }^{13}$ C NMR (101 MHz, Chloroform-d): $\delta$ 147.31, 141.70, 139.66, 130.48, 128.67, 128.22, 127.62, 126.66, 117.70, 116.42 .

IR (ATR): $v\left(\mathrm{~cm}^{-1}\right)$ 3478, 3385, 3080, 3054, 3025, 1608, 1492, 1443, 905, 807, 778, 709, 695, 590.

HRMS (ESI/QTOF): calcd for $\mathrm{C}_{22} \mathrm{H}_{20} \mathrm{~N}^{+}[\mathrm{M}+\mathrm{H}]^{+}:$298.1590; found 298.1591 .

\section{2,6-Bis(1-phenylvinyl)-4-(trifluoromethyl)aniline 12e}<smiles>C=C(c1ccccc1)c1cc(C(F)(F)F)cc(C(=C)c2ccccc2)c1N</smiles>

Yield: $79 \%$ (Route B)

Appearance: colourless oil

${ }^{1}$ H NMR (400 MHz, Chloroform-d): $\delta 7.31$ (s, 2H), $7.30-7.19$ (m, 10H), 5.78 (d, $\left.J=1.2 \mathrm{~Hz}, 2 \mathrm{H}\right), 5.34$ (d, $J=1.2 \mathrm{~Hz}, 2 \mathrm{H}), 3.74$ (bs, $2 \mathrm{H}$ ).

${ }^{13}$ C NMR (101 MHz, Chloroform-d): $\delta$ 146.24, 144.75, 138.74, 128.86, 128.59, 127.37 (q, J=4.04 Hz, 2C), 126.97, 126.56, 124.99 (q, $J=270.8 \mathrm{~Hz}), 119.41$ (q, $J=32.6 \mathrm{~Hz}), 117.40$.

${ }^{19}$ F NMR (376 MHz, Chloroform- $d$ ): $\delta$-60.88.

IR (ATR): $v\left(\mathrm{~cm}^{-1}\right)$ 3490, 3394, 3059, 3027, 2957, 1619, 1356, 1316, 1295, 1277, 1152, 1111, 900, 696.

HRMS (ESI/QTOF): calcd for $\mathrm{C}_{23} \mathrm{H}_{19} \mathrm{~F}_{3} \mathrm{~N}^{+}[\mathrm{M}+\mathrm{H}]^{+}$: 366.1464; found 366.1467 .

\section{2,6-Bis(1-(4-(tert-butyl)phenyl)vinyl)-4-methylaniline $12 f$}

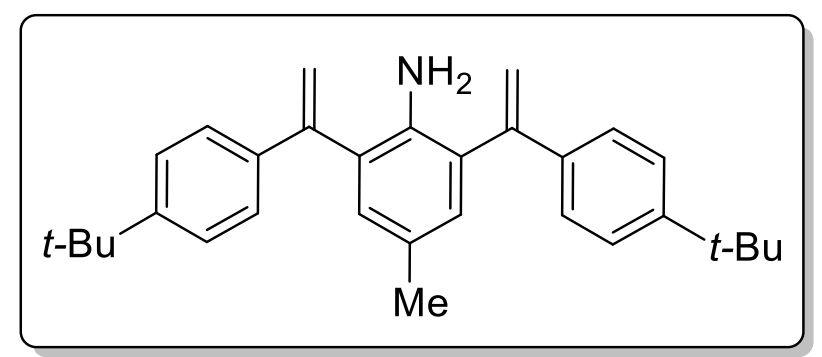

Yield: 75\% (Route B)

Appearance: yellow oil

${ }^{1}$ H NMR (400 MHz, Chloroform- $d$ ): $\delta 7.38-7.30(\mathrm{~m}, 8 \mathrm{H}), 6.95(\mathrm{~d}, J=2.3 \mathrm{~Hz}, 2 \mathrm{H}), 5.81(\mathrm{t}, J=1.6 \mathrm{~Hz}$, $2 \mathrm{H}), 5.34(\mathrm{t}, J=1.6 \mathrm{~Hz}, 2 \mathrm{H}), 3.45(\mathrm{bs}, 2 \mathrm{H}), 2.29(\mathrm{~d}, J=1.9 \mathrm{~Hz}, 3 \mathrm{H}), 1.36-1.29(\mathrm{~m}, 18 \mathrm{H})$.

${ }^{13}$ C NMR (101 MHz, Chloroform-d): $\delta$ 151.18, 147.09, 139.26, 136.72, 130.77, 127.96, 126.73, 126.33, 125.55, 115.36, 34.69, 31.43, 20.54. 
IR (ATR): $v\left(\mathrm{~cm}^{-1}\right)$ 3477, 3384, 3085, 3030, 2961, 2904, 2867, 1614, 1510, 1458, 1363, 1269, 899, 842.

HRMS (ESI/QTOF): calcd for $\mathrm{C}_{31} \mathrm{H}_{38} \mathrm{~N}^{+}[\mathrm{M}+\mathrm{H}]^{+}$: 424.2999; found 424.3005 .

\section{4-Methyl-2,6-bis(1-(naphthalen-2-yl)vinyl)aniline 12g}

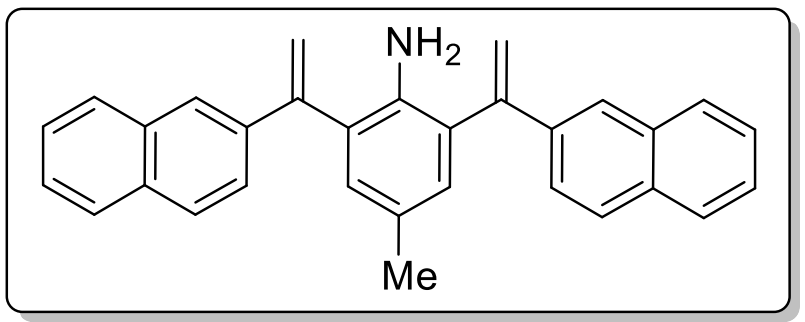

Yield: $51 \%$ (Route B)

Appearance: yellow solid

${ }^{1}$ H NMR (400 MHz, Chloroform- $\left.\boldsymbol{d}\right): \delta 7.82-7.73(\mathrm{~m}, 8 \mathrm{H}), 7.61(\mathrm{dd}, J=8.6,1.8 \mathrm{~Hz}, 2 \mathrm{H}), 7.48-7.40$ (m, 4H), 7.06 (s, 2H), 5.93 (d, $J=1.4 \mathrm{~Hz}, 2 \mathrm{H}), 5.49$ (d, $J=1.4 \mathrm{~Hz}, 2 \mathrm{H}), 3.42$ (bs, 2H), 2.34 (s, 3H).

${ }^{13}$ C NMR (101 MHz, Chloroform-d): $\delta$ 147.45, 139.40, 137.07, 133.56, 133.26, 131.19, 128.47, 128.32, $127.90,127.69,127.02,126.32,126.25,125.99,124.55,116.82,20.61$.

IR (ATR): $v\left(\mathrm{~cm}^{-1}\right) 3473,3382,3054,3017,2916,2860,1614,1595,1504,1458,1270,1242,897,859$, $820,751,475$.

HRMS (ESI/QTOF): calcd for $\mathrm{C}_{31} \mathrm{H}_{26} \mathrm{~N}^{+}[\mathrm{M}+\mathrm{H}]^{+}$: 412.2060; found 412.2060.

Melting point: $131-134{ }^{\circ} \mathrm{C}$

\section{$\underline{\text { 2,6-Bis(1-(3,5-difluorophenyl)vinyl)-4-methylaniline 12i }}$}

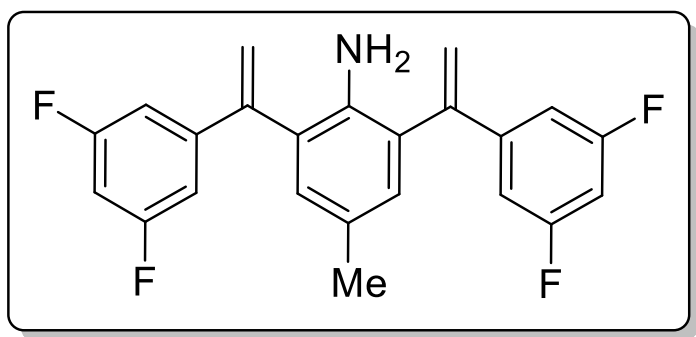

Yield: $89 \%$ (Route B)

Appearance: off-white solid

${ }^{1} \mathrm{H}$ NMR (400 MHz, Chloroform- $d$ ): $\delta 6.94-6.83(\mathrm{~m}, 6 \mathrm{H}), 6.73(\mathrm{tt}, J=8.7,2.3 \mathrm{~Hz}, 2 \mathrm{H}), 5.85(\mathrm{~d}, J=$ $1.0 \mathrm{~Hz}, 2 \mathrm{H}), 5.46$ (s, 2H), 3.37 (bs, 2H), 2.28 (s, 3H).

${ }^{13}$ C NMR (101 MHz, Chloroform- $d$ ): $\delta 163.31$ (dd, $\left.J=248.1,12.9 \mathrm{~Hz}\right), 145.46(\mathrm{t}, J=2.6 \mathrm{~Hz}), 143.23$ (t, $J=9.0 \mathrm{~Hz}), 138.91,131.32,127.37,126.70,118.36,109.55(\mathrm{dd}, J=19.2,7.07 \mathrm{~Hz}), 103.52(\mathrm{t}, J=25.7$ $\mathrm{Hz}), 20.46$. 
${ }^{19}$ F NMR (376 MHz, Chloroform-d): $\delta$-109.81.

IR (ATR): $v\left(\mathrm{~cm}^{-1}\right) 3479,3390,3091,2920,2863,1619,1585,1460,1432,1351,1328,1289,1118,988$, 916, 861.

HRMS (ESI/QTOF): calcd for $\mathrm{C}_{23} \mathrm{H}_{18} \mathrm{~F}_{4} \mathrm{~N}^{+}[\mathrm{M}+\mathrm{H}]^{+}:$384.1370; found 384.1375.

Melting point: $62.5-64.9^{\circ} \mathrm{C}$

\section{4-Methyl-2,6-bis((R)-1-phenylethyl)aniline 1a}

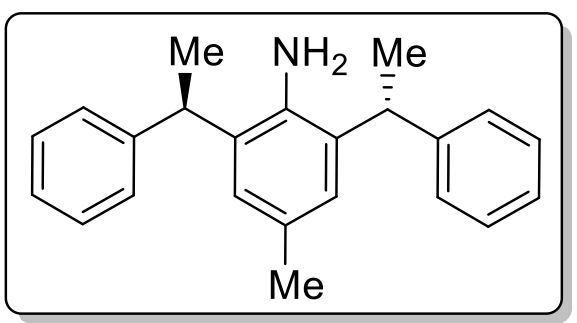

Prepared according to GP1. Spectroscopic data matched those previously reported in the literature. ${ }^{6}$

${ }^{1}$ H NMR (400 MHz, Chloroform- $d$ ): $\delta 7.28-7.21$ (m, 4H), $7.19-7.12$ (m, 6H), 7.06 (s, 2H), 3.99 (q, $J=7.1 \mathrm{~Hz}, 2 \mathrm{H}), 3.22$ (bs, 2H), 2.37 (s, 3H), 1.59 (d, $J=7.1 \mathrm{~Hz}, 6 \mathrm{H})$.

${ }^{13}$ C NMR (101 MHz, Chloroform-d): $\delta$ 146.07, 139.74, 130.04, 128.80, 127.50, 126.83, 126.37, 126.19, $40.53,22.49,21.33$.

e.r.: 96.4:0:3.6 (R,R):(S,S):(meso) (SFC, Chiracel OD-H, 5\% MeOH/CO $2, \lambda=220 \mathrm{~nm}, 4 \mathrm{~mL} / \mathrm{min}, 150$ bar outlet, $\mathrm{t}_{\mathrm{R}}(R, R)=6.12 \mathrm{~min} ; \mathrm{t}_{\mathrm{R}}($ meso $\left.)=4.94 \mathrm{~min}\right)$.

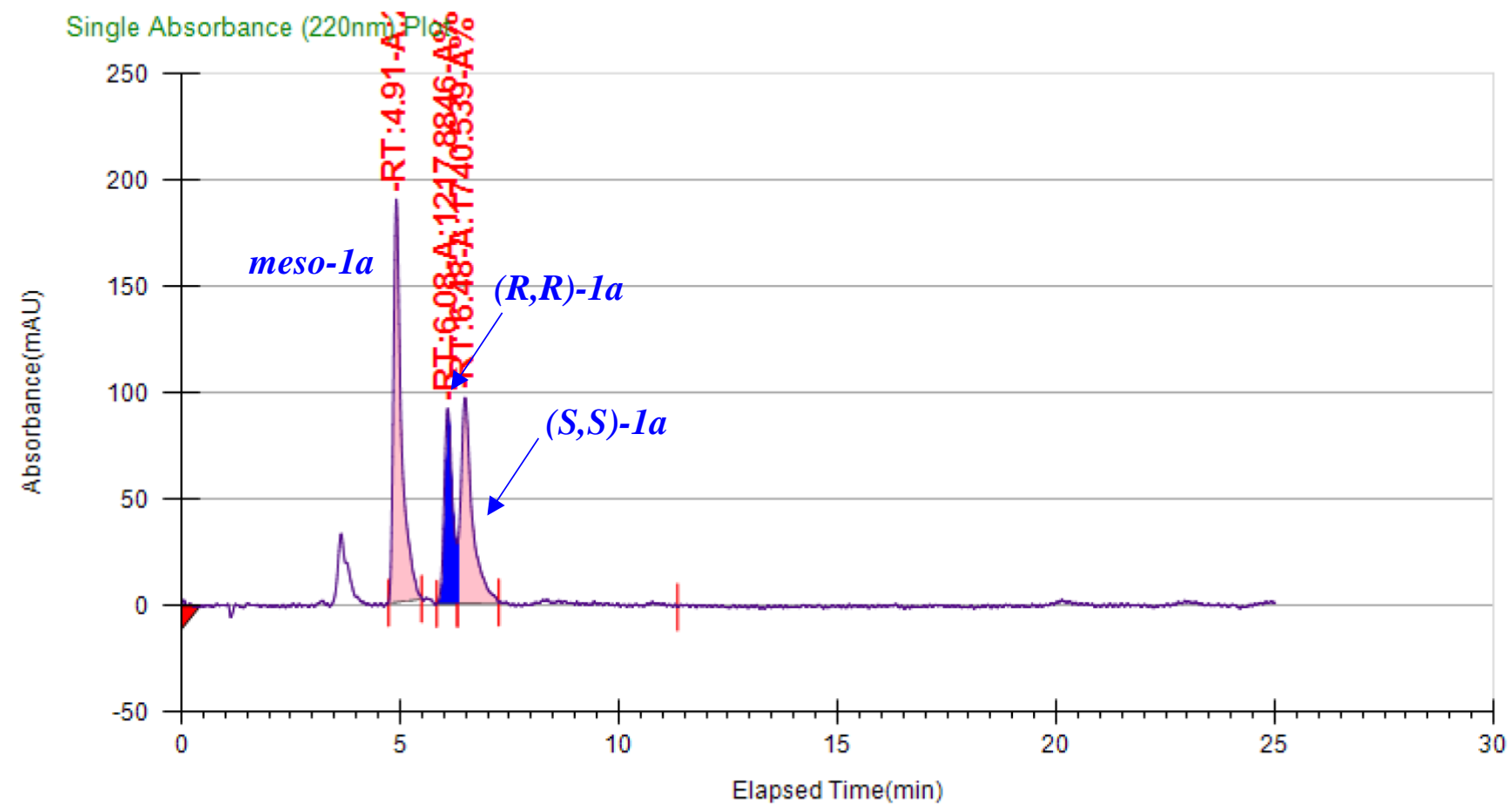




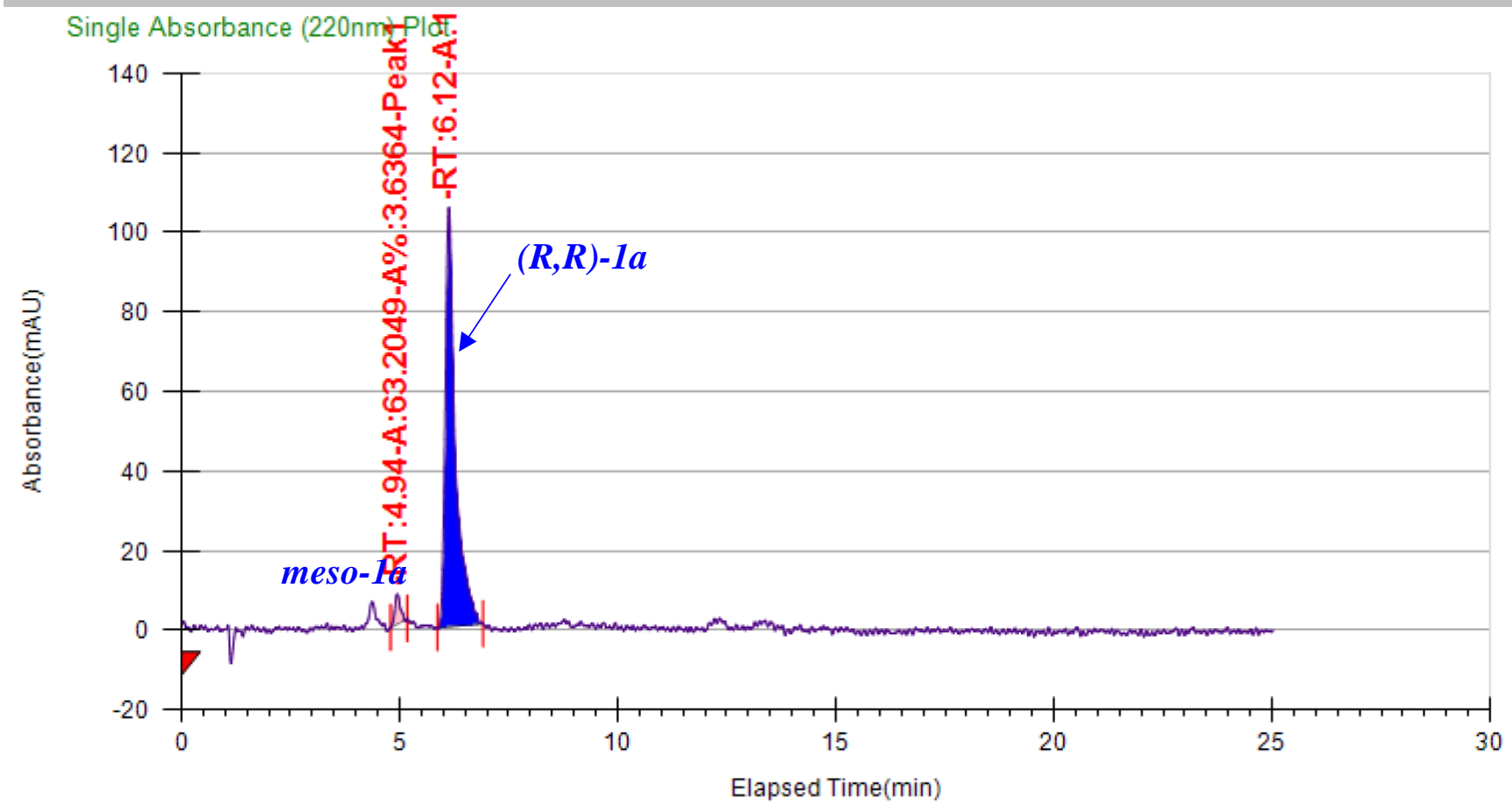

\begin{tabular}{|l|l|l|l|l|l|}
\hline Peak No & \% Area & Area & Ret. Time & Height & Cap. Factor \\
\hline 1 & 3.6364 & 63.2049 & $4.94 \mathrm{~min}$ & 7.4479 & 0 \\
\hline 2 & 96.3636 & 1674.9143 & $6.12 \mathrm{~min}$ & 105.4412 & 0 \\
\hline
\end{tabular}

\section{$\underline{\text { 2,6-Bis }((R)-1-p h e n y l e t h y l) \text { aniline } 1 \mathrm{~b}}$}

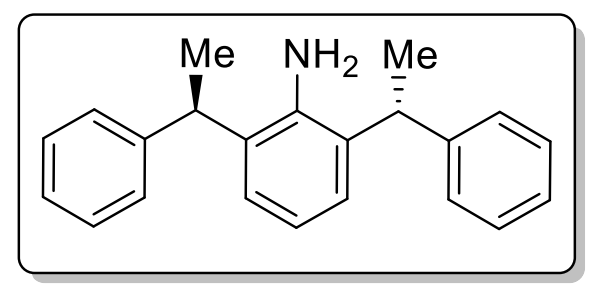

Prepared according to $\underline{\mathbf{G P 1}}$.

Yield: $97 \%$

Appearance: off-white solid

${ }^{1}$ H NMR (400 MHz, Chloroform-d): $\delta 7.26(\mathrm{ddd}, J=9.7,5.9,1.6 \mathrm{~Hz}, 6 \mathrm{H}), 7.21-7.12(\mathrm{~m}, 4 \mathrm{H}), 6.94$ $(\mathrm{td}, J=7.7,1.5 \mathrm{~Hz}, 1 \mathrm{H}), 4.00(\mathrm{q}, J=7.1 \mathrm{~Hz}, 2 \mathrm{H}), 3.37(\mathrm{bs}, 2 \mathrm{H}), 1.61(\mathrm{dd}, J=7.2,1.6 \mathrm{~Hz}, 6 \mathrm{H})$.

${ }^{13}$ C NMR (101 MHz, Chloroform-d): $\delta$ 145.96, 142.29, 129.77, 128.82, 127.48, 126.43, 125.53, 117.90, $40.56,22.54$.

IR (ATR): $v\left(\mathrm{~cm}^{-1}\right)$ 3477, 3385, 3059, 3023, 2965, 2931, 2872, 1620, 1443, 749, 699.

HRMS (ESI/QTOF): calcd for $\mathrm{C}_{22} \mathrm{H}_{23} \mathrm{~N}^{+}[\mathrm{M}+\mathrm{H}]^{+}:$302.1904; found 302.1909.

QD: $+57.0\left(c=1.0, \mathrm{CHCl}_{3}\right)$ 


\section{Melting point: $70.0-73.4{ }^{\circ} \mathrm{C}$}

e.r.: $>99: 1$ (Chiralpak IG, Hexane/IPA $=99.5 / 0.5, \lambda=230 \mathrm{~nm}, 1.00 \mathrm{~mL} / \mathrm{min}, \mathrm{t}_{\mathrm{R}}(R, R)=6.21 \mathrm{~min}$ ).

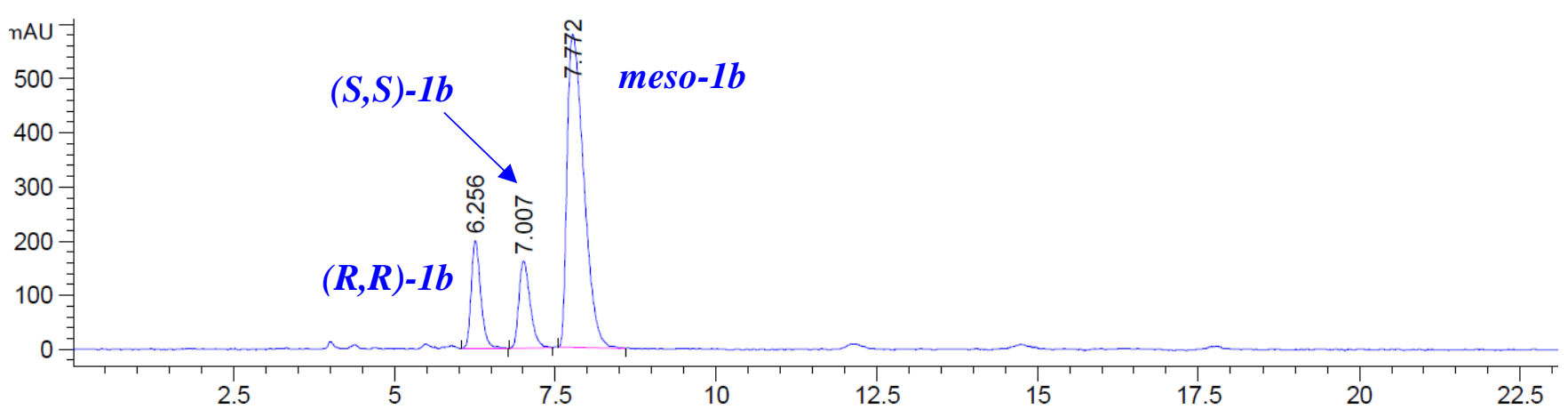

Peak RetTime Type Width Area Height Area

$\begin{array}{ccccccc}\# & {[\mathrm{~min}]} & {[\mathrm{min}]} & {\left[\mathrm{mAU}^{*} \mathrm{~s}\right]} & {[\mathrm{mAU}]} & \frac{\circ}{0} \\ -1 & 6.256 & \text { VB } & 0.1587 & 2072.99976 & 200.42133 & 14.3377 \\ 2 & 7.007 & \text { BB } & 0.1843 & 1967.58374 & 161.28534 & 13.6086 \\ 3 & 7.772 & \text { BB } & 0.2860 & 1.04178 e 4 & 578.66675 & 72.0537\end{array}$

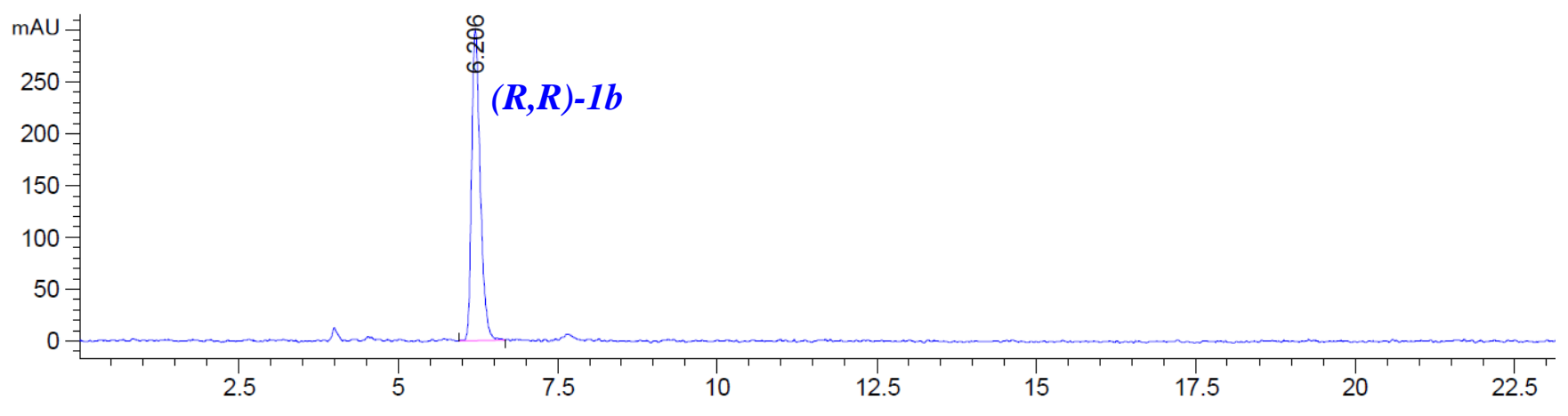

Peak RetTime Type Width Area Height Area

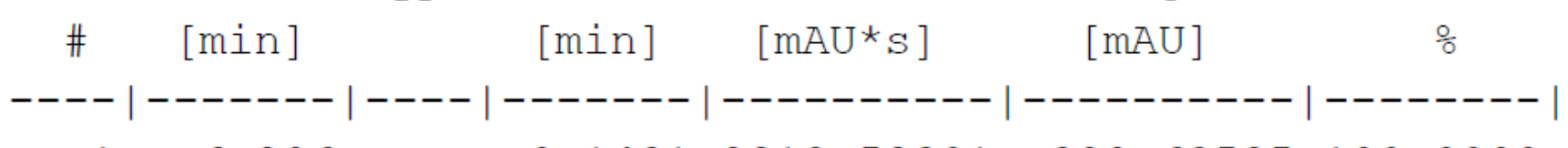

$16.206 \mathrm{BB} \quad 0.14312812 .58301 \quad 300.63535100 .0000$ 


\section{4-Methoxy-2,6-bis((R)-1-phenylethyl)aniline 1c}

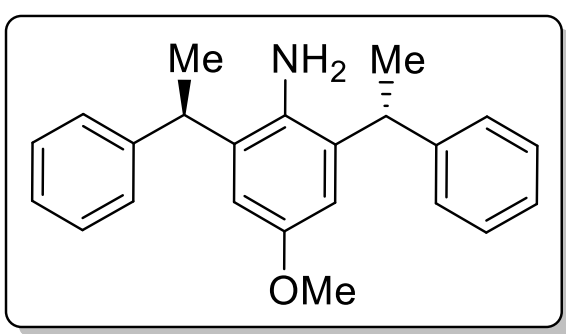

Prepared according to $\underline{\mathbf{G P 1}}$. Spectroscopic data matched those previously reported in the literature. ${ }^{7}$ ${ }^{1}$ H NMR (400 MHz, Chloroform-d): $\delta 7.29$ - 7.23 (m, 4H), 7.20 - 7.13 (m, 6H), 6.88 (s, 2H), 4.03 (q, $J=7.1 \mathrm{~Hz}, 2 \mathrm{H}), 3.85$ (s, 3H), 3.09 (bs, 2H), 1.60 (d, $J=7.2 \mathrm{~Hz}, 6 \mathrm{H})$.

${ }^{13}$ C NMR (101 MHz, Chloroform-d): $\delta$ 152.44, 145.73, 136.02, 131.61, 128.83, 127.49, 126.45, 111.46, $55.87,40.68,22.46$.

e.r.: $>99: 1$ (Chiralpak IB, Hexane/IPA $=99.5 / 0.5, \lambda=306 \mathrm{~nm}, 1.00 \mathrm{~mL} / \mathrm{min}, \mathrm{t}_{\mathrm{R}}(R, R)=9.60 \mathrm{~min}$ ).

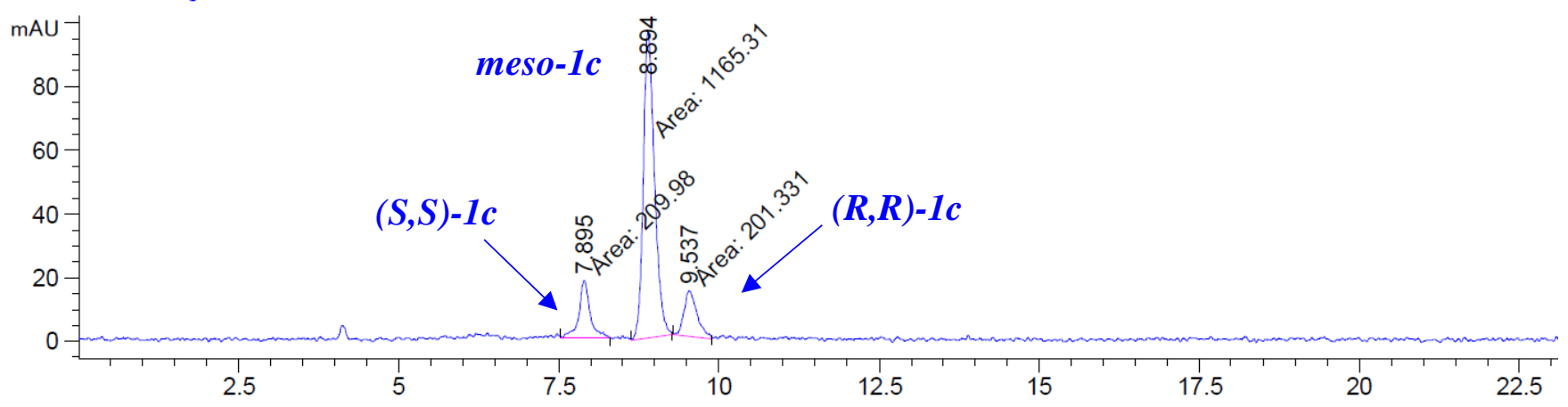

\begin{tabular}{|c|c|c|c|c|c|c|}
\hline $\begin{array}{c}\text { Peak } \\
\#\end{array}$ & $\begin{array}{c}\text { RetTime } \\
\text { [min] }\end{array}$ & Type & $\begin{array}{l}\text { Width } \\
\text { [min] }\end{array}$ & $\begin{array}{c}\text { Area } \\
{\left[\mathrm{mAU}^{*} \mathrm{~s}\right]}\end{array}$ & $\begin{array}{l}\text { Height } \\
{[\mathrm{mAU}]}\end{array}$ & $\begin{array}{c}\text { Area } \\
\quad \frac{\circ}{\circ}\end{array}$ \\
\hline & 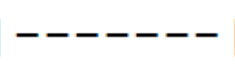 & & & ---------- & & 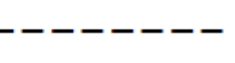 \\
\hline 1 & 7.895 & $\mathrm{MM}$ & 0.1963 & 209.98013 & 17.82952 & 13.3184 \\
\hline 2 & 8.894 & $\mathrm{MM}$ & 0.2015 & 1165.30676 & 96.36832 & 73.9118 \\
\hline 3 & 9.537 & MM & 0.2370 & 201.33141 & 838 & 7698 \\
\hline
\end{tabular}

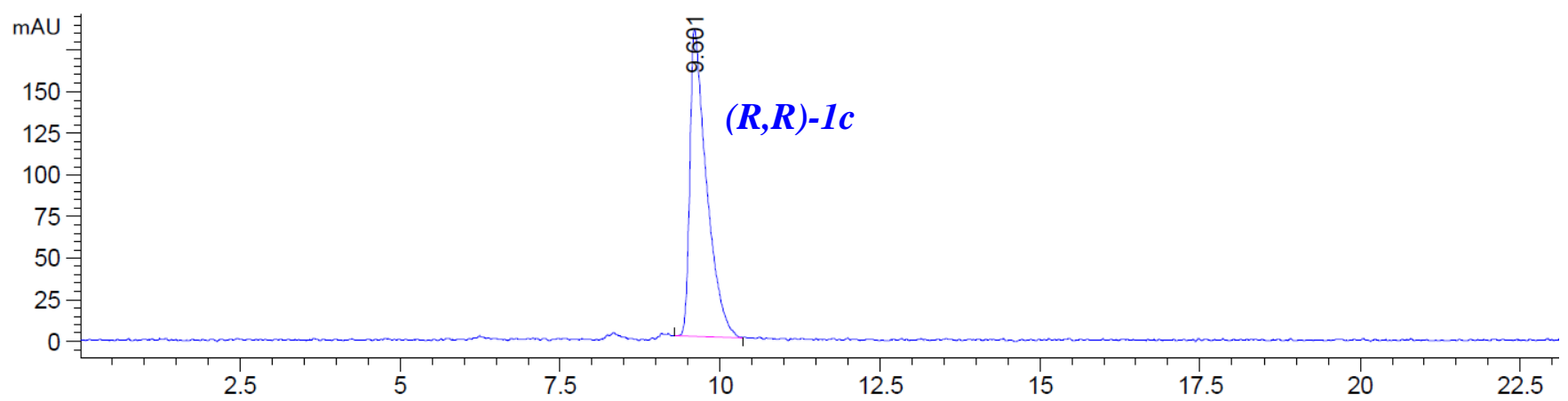




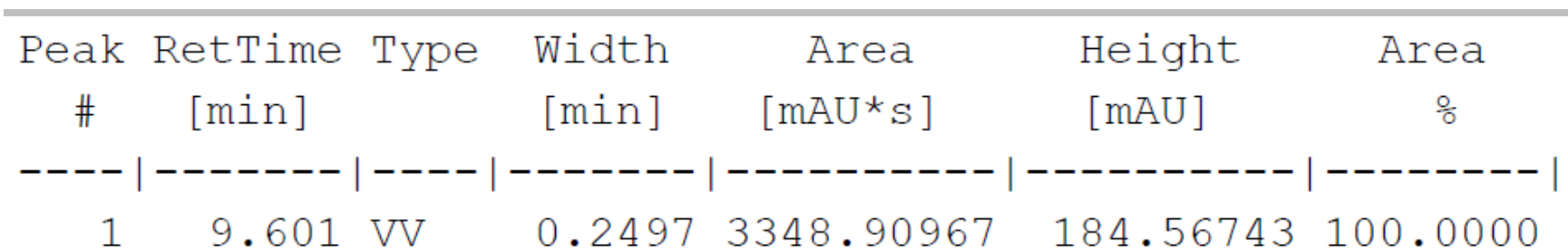

\section{4-(Tert-butyl)-2,6-bis((R)-1-phenylethyl)aniline 1d}

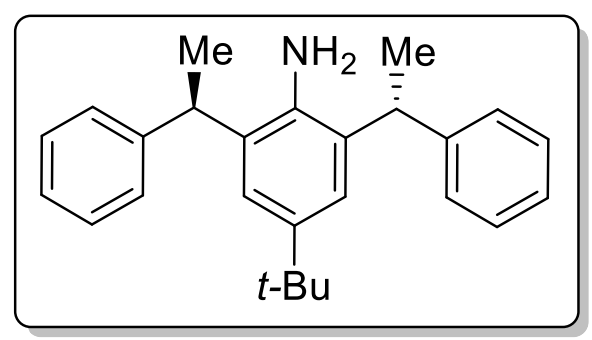

Prepared according to $\underline{\text { GP1 }}$. Spectroscopic data matched those previously reported in the literature. ${ }^{5}$

${ }^{1} \mathrm{H}$ NMR (400 MHz, Chloroform-d): $\delta 7.29$ (s, 2H), 7.24 (s, 2H), 7.22 (s, 2H), 7.19-7.12 (m, 6H), 4.03 (q, J=7.2 Hz, 2H), 2.17 (bs, 2H), 1.61 (d, J=7.1 Hz, 6H), 1.37 (s, 9H).

${ }^{13}$ C NMR (101 MHz, Chloroform-d): $\delta$ 145.56, 128.77, 127.65, 126.45, 122.84, 40.55, 34.60, 31.75, $22.56 \mathrm{ppm}$

e.r.: 97.1:1.6:1.3 $(R, R):(S, S):\left(\right.$ meso) (Chiralpak IA, Hexane/IPA $=99.5 / 0.5, \lambda=210 \mathrm{~nm}, 1.00 \mathrm{~mL} / \mathrm{min}, \mathrm{t}_{\mathrm{R}}$ $(R, R)=4.63 \mathrm{~min} ; \mathrm{t}_{\mathrm{R}}(S, S)=5.22 \mathrm{~min} ; \mathrm{t}_{\mathrm{R}}($ meso $\left.)=5.42 \mathrm{~min}\right)$.

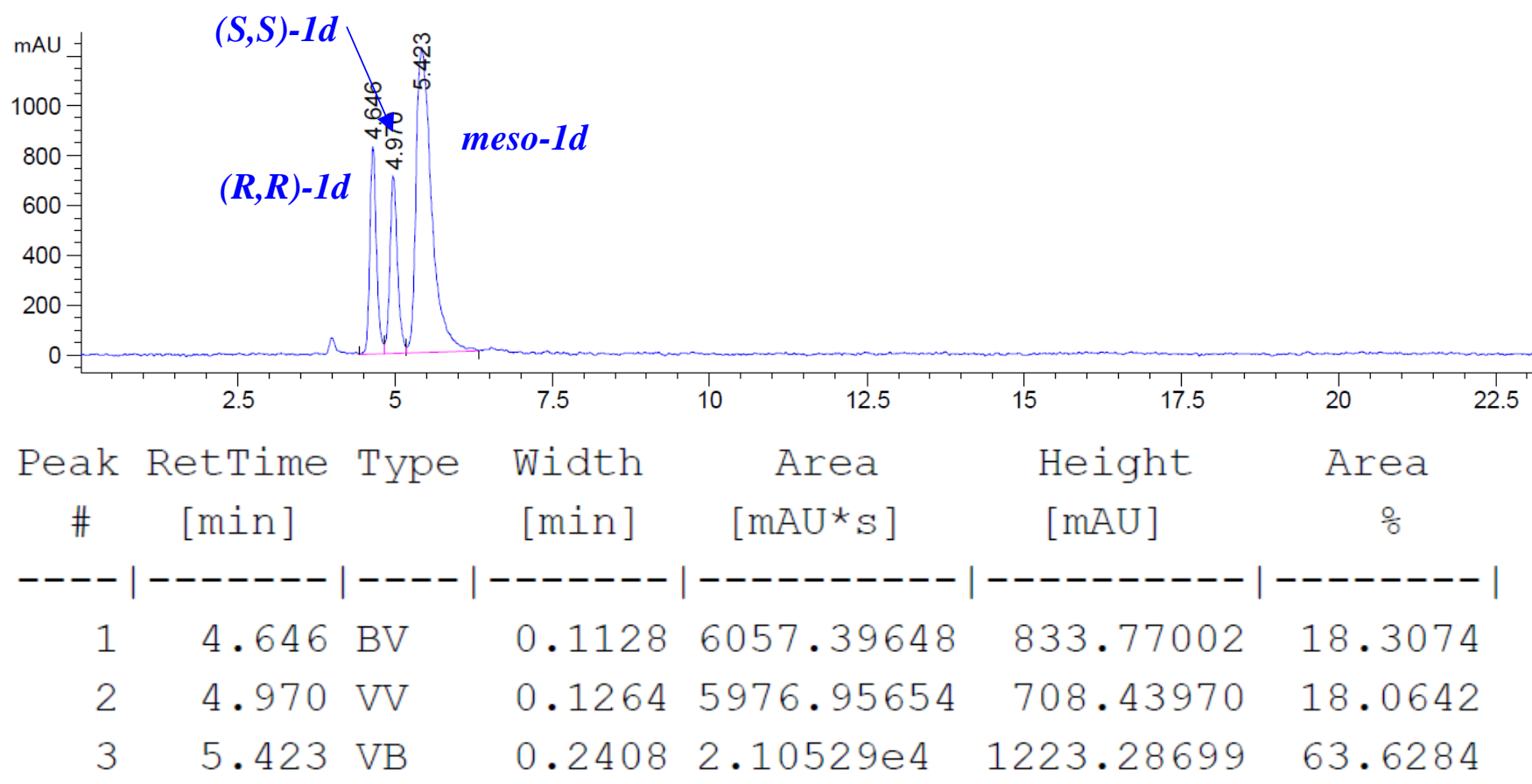




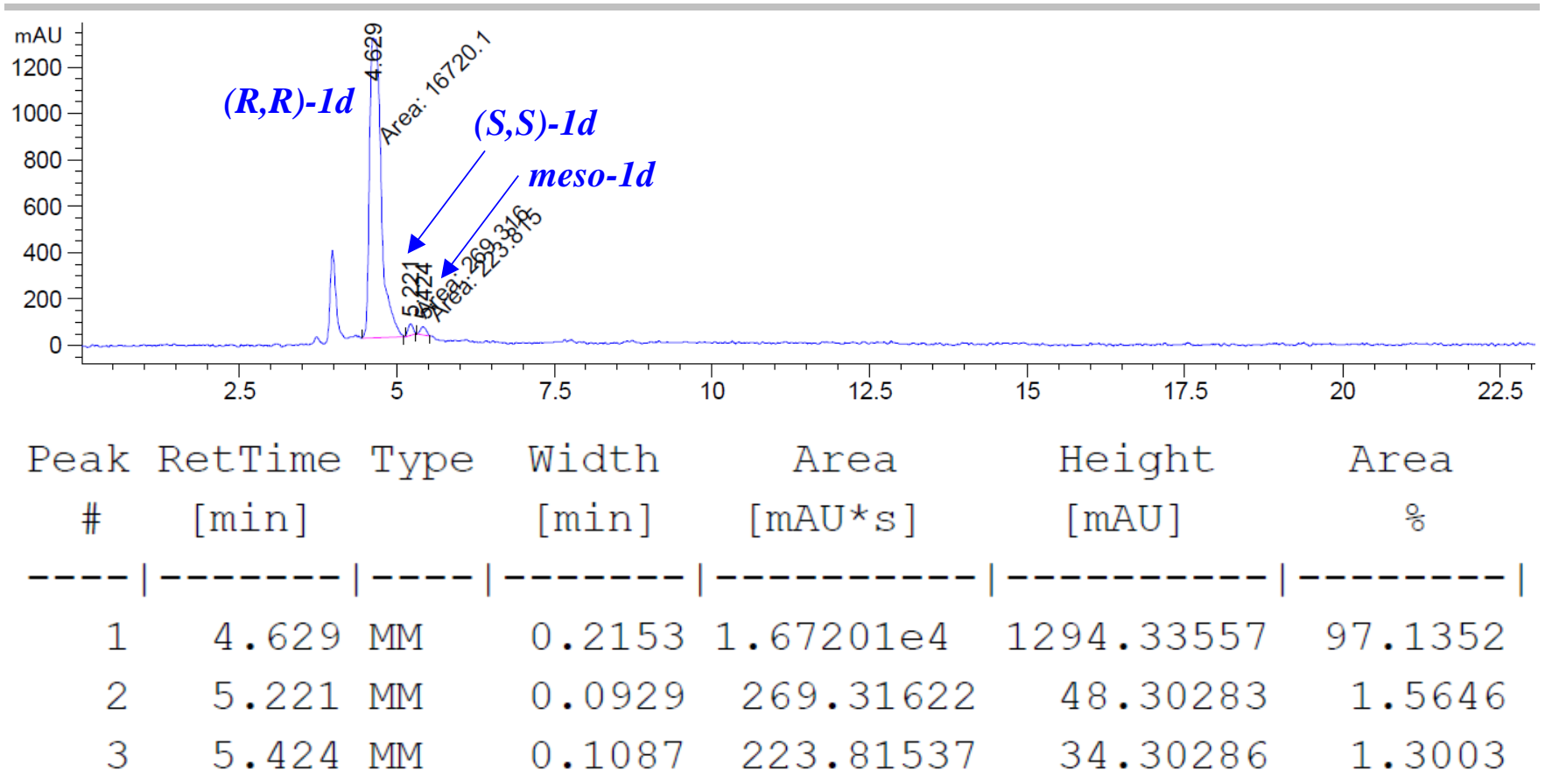

\section{$\underline{\text { 2,6-Bis((R)-1-phenylethyl)-4-(trifluoromethyl)aniline 1e }}$}

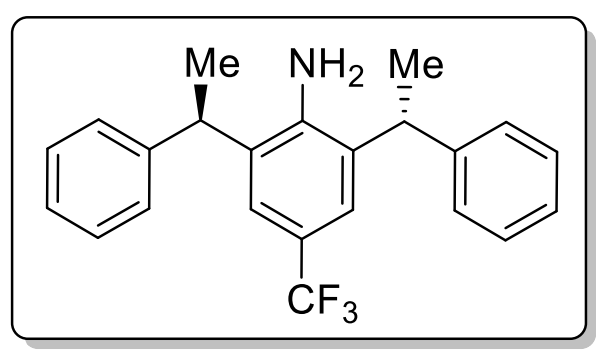

Prepared according to $\underline{\text { GP1 }}$.

Yield: $66 \%$

Appearance: yellow oil

${ }^{1}$ H NMR (400 MHz, Chloroform-d): $\delta 7.52$ (s, 2H), 7.27 (d, J=6.9 Hz, 2H), 7.22 - 7.16 (m, 2H), 7.14 - $7.08(\mathrm{~m}, 4 \mathrm{H}), 3.93(\mathrm{q}, J=7.1 \mathrm{~Hz}, 2 \mathrm{H}), 3.65(\mathrm{bs}, 2 \mathrm{H}), 1.62(\mathrm{~d}, J=7.2 \mathrm{~Hz}, 6 \mathrm{H})$.

${ }^{13}$ C NMR (101 MHz, Chloroform-d): $\delta$ 145.41, 144.92, 129.08, 129.05, 127.33, 126.82, 125.55 (q, $J=$ $271.7 \mathrm{~Hz}), 122.64$ (q, $J=3.9 \mathrm{~Hz}, 2 \mathrm{C}), 119.34$ (q, $J=31.9 \mathrm{~Hz}), 40.65,22.56$.

${ }^{19}$ F NMR (376 MHz, Chloroform-d): $\delta-60.53$.

IR (ATR): $v\left(\mathrm{~cm}^{-1}\right)$ 3492, 3402, 3026, 2969, 2933, 2875, 1626, 1450, 1325, 1102, 894, 700.

HRMS (ESI/QTOF): calcd for $\mathrm{C}_{23} \mathrm{H}_{23} \mathrm{~F}_{3} \mathrm{~N}^{+}[\mathrm{M}+\mathrm{H}]^{+}:$370.1778; found 370.1783 .

QD: $+24.0\left(c=0.8, \mathrm{CHCl}_{3}\right)$

e.r.: $>99: 1$ (Chiralpak IB, Hexane/IPA $=99.5 / 0.5, \lambda=254 \mathrm{~nm}, 1.00 \mathrm{~mL} / \mathrm{min}, \mathrm{t}_{\mathrm{R}}(R, R)=8.69 \mathrm{~min}$ ). 

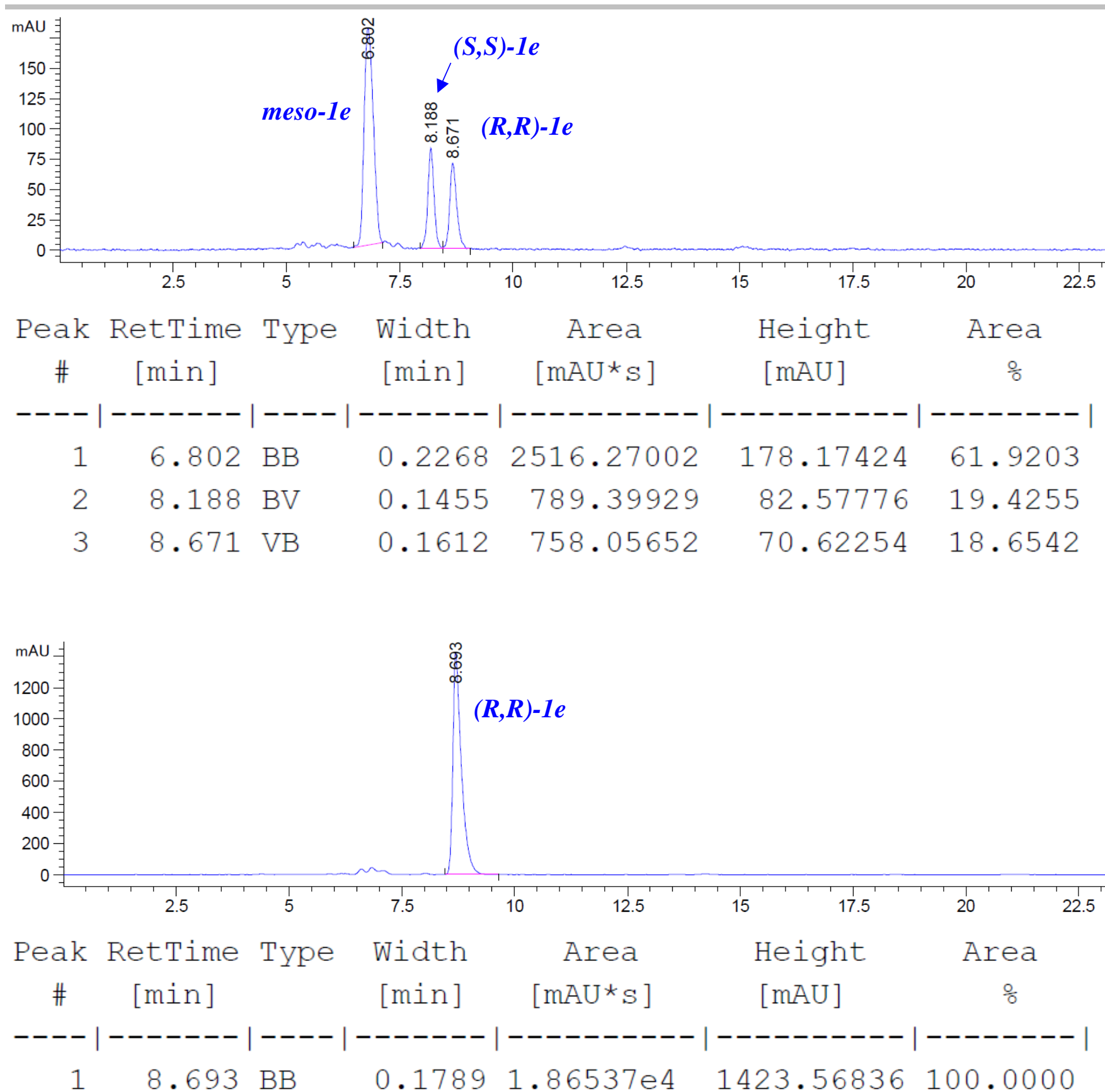

2,6-Bis((R)-1-(4-(tert-butyl)phenyl)ethyl)-4-methylaniline If

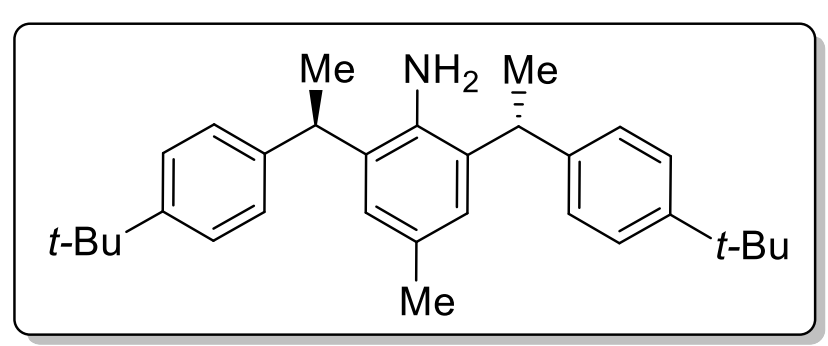

Prepared according to $\underline{\text { GP1 }}$.

Yield: $52 \%$ 


\section{Appearance: white solid}

${ }^{1}$ H NMR (400 MHz, Chloroform-d): $\delta 7.27(\mathrm{~d}, J=8.7 \mathrm{~Hz}, 4 \mathrm{H}), 7.12-7.04(\mathrm{~m}, 6 \mathrm{H}), 4.00(\mathrm{q}, J=7.2$ $\mathrm{Hz}, 2 \mathrm{H}), 3.28$ (bs, 2H), 2.38 (s, 3H), 1.60 (d, $J=7.1 \mathrm{~Hz}, 6 \mathrm{H}), 1.29$ (s, 18H).

${ }^{13}$ C NMR (101 MHz, Chloroform-d): $\delta$ 148.95, 142.78, 139.72, 130.37, 127.08, 126.87, 126.06, 125.62, 39.90, 34.47, 31.52, 22.47, 21.35.

IR (ATR): $v\left(\mathrm{~cm}^{-1}\right)$ 3473, 3378, 3023, 2962, 2905, 2868, 1507, 1268, 864.

HRMS (ESI/QTOF): calcd for $\mathrm{C}_{31} \mathrm{H}_{42} \mathrm{~N}^{+}[\mathrm{M}+\mathrm{H}]^{+}$: 428.3312 ; found 428.3319 .

aD: $+4.50\left(c=1.0, \mathrm{CHCl}_{3}\right)$

Melting point: $60.4-63.0{ }^{\circ} \mathrm{C}$

e.r.: 97.3:2.3:0 (R,R):(S,S):(meso) (Chiralpak IA, Hexane/IPA $=99.5 / 0.5, \lambda=230 \mathrm{~nm}, 1.00 \mathrm{~mL} / \mathrm{min}, \mathrm{t}_{\mathrm{R}}$ $\left.(R, R)=5.14 \min , \mathrm{t}_{\mathrm{R}}(S, S)=4.74 \mathrm{~min}\right)$.

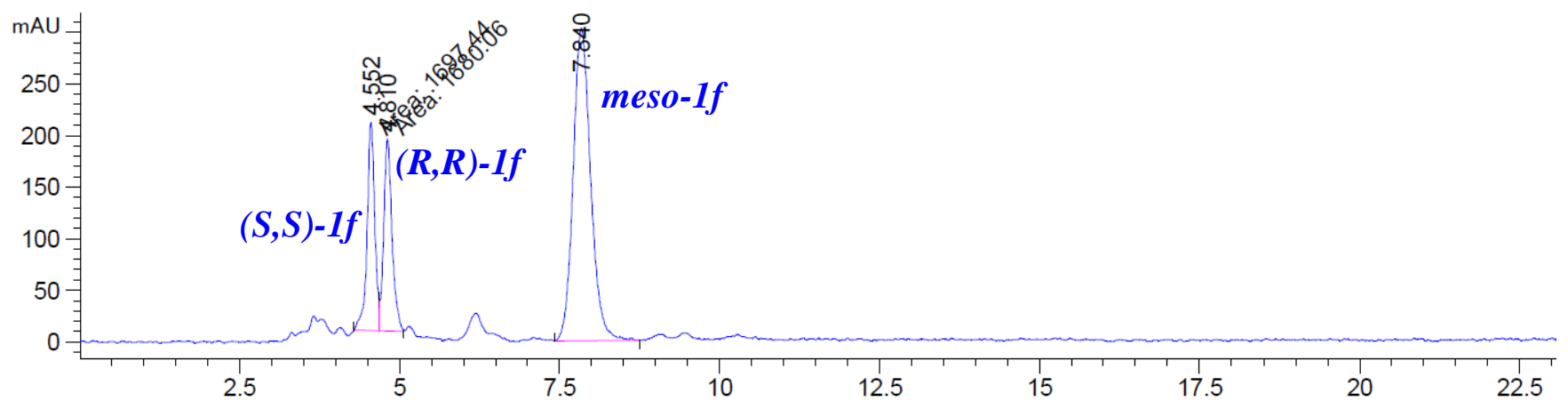

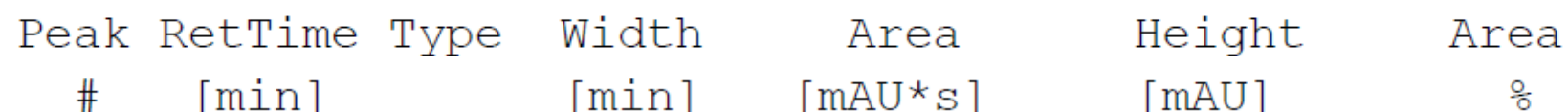

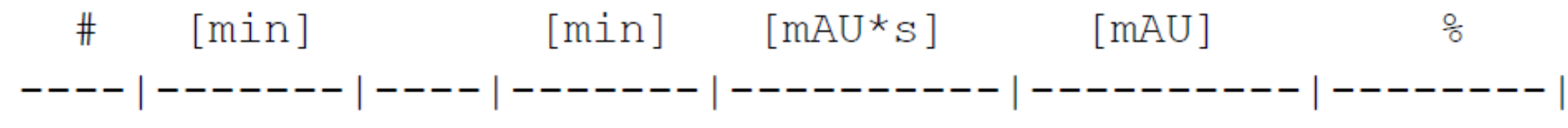

$\begin{array}{lllllll}1 & 4.552 & \mathrm{MF} & 0.1399 & 1697.44360 & 202.23538 & 18.5701 \\ 2 & 4.810 & \mathrm{FM} & 0.1506 & 1680.06128 & 185.89156 & 18.3799 \\ 3 & 7.840 & \mathrm{BB} & 0.2862 & 5763.22852 & 302.65479 & 63.0500\end{array}$

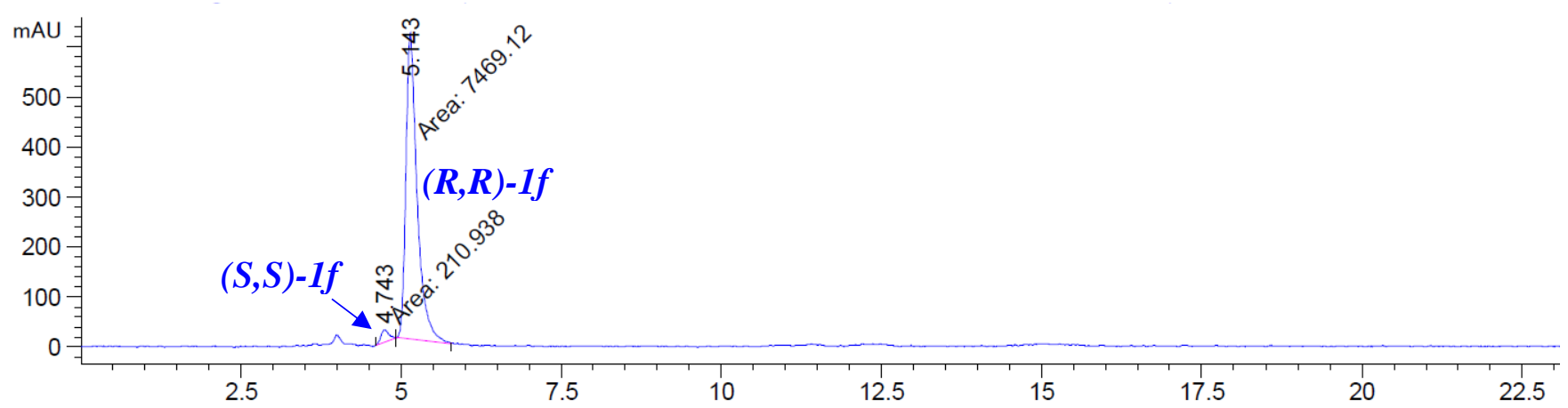




\begin{tabular}{|c|c|c|c|c|c|c|}
\hline $\begin{array}{c}\text { Peak } \\
\text { \# }\end{array}$ & $\begin{array}{c}\text { RetTime } \\
\text { [min] }\end{array}$ & Type & $\begin{array}{l}\text { Width } \\
\text { [min] }\end{array}$ & $\begin{array}{c}\text { Area } \\
{\left[\mathrm{mAU}{ }^{*} \mathrm{~s}\right]}\end{array}$ & $\begin{array}{l}\text { Height } \\
{[\mathrm{mAU}]}\end{array}$ & $\begin{array}{c}\text { Area } \\
\frac{\circ}{0}\end{array}$ \\
\hline . & ----- & . & ---- & ------- & --------- & $-------\mid$ \\
\hline 1 & 4.743 & MM & 0.1414 & 210.93825 & 24.86070 & 2.7466 \\
\hline 2 & 5.143 & MM & 0.2034 & 7469.11621 & 612.03857 & 97.2534 \\
\hline
\end{tabular}

\section{4-Methyl-2,6-bis((R)-1-(naphthalen-2-yl)ethyl)aniline 1g}

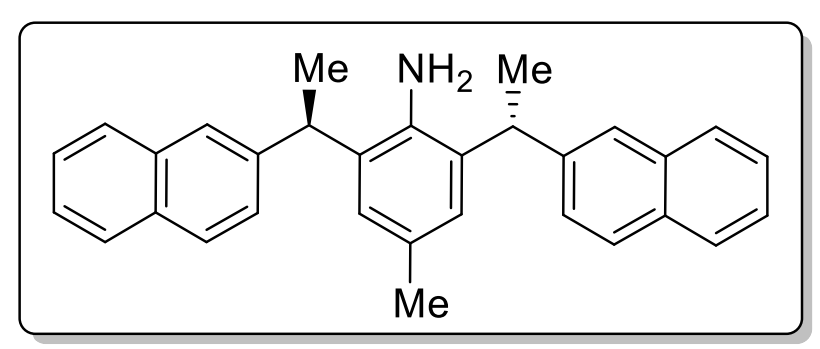

Prepared according to $\underline{\text { GP1. }}$.

Yield: $98 \%$

Appearance: white solid

${ }^{1}$ H NMR (400 MHz, Chloroform- $\left.d\right): \delta 7.80-7.71(\mathrm{~m}, 6 \mathrm{H}), 7.62(\mathrm{~d}, J=1.6 \mathrm{~Hz}, 2 \mathrm{H}), 7.47-7.38(\mathrm{~m}$, $4 \mathrm{H}), 7.28(\mathrm{dd}, J=8.5,1.8 \mathrm{~Hz}, 2 \mathrm{H}), 7.14(\mathrm{~s}, 2 \mathrm{H}), 4.16(\mathrm{q}, J=7.1 \mathrm{~Hz}, 2 \mathrm{H}), 3.32(\mathrm{bs}, 2 \mathrm{H}), 2.42(\mathrm{~s}, 3 \mathrm{H})$, $1.68(\mathrm{~d}, J=7.1 \mathrm{~Hz}, 6 \mathrm{H})$.

${ }^{13}$ C NMR (101 MHz, Chloroform-d): $\delta$ 143.48, 140.02, 133.72, 132.37, 130.01, 128.58, 127.77, 127.74 , $126.99,126.52,126.38,126.09,125.52,125.45,40.77,22.22,21.37$.

IR (ATR): $v\left(\mathrm{~cm}^{-1}\right)$ 3473, 3380, 3053, 2966, 2930, 2871, 1467, 1454, 908, 819, 746, 732, 478.

HRMS (ESI/QTOF): calcd for $\mathrm{C}_{31} \mathrm{H}_{30} \mathrm{~N}^{+}[\mathrm{M}+\mathrm{H}]^{+}: 416.2373$; found 436.2371 .

QD: $+106\left(c=1.0, \mathrm{CHCl}_{3}\right)$

Melting point: $72.4-75.3^{\circ} \mathrm{C}$

e.r.: $>99: 1$ (Chiralpak IF, Hexane/IPA $\left.=99.5 / 0.5, \lambda=230 \mathrm{~nm}, 1.00 \mathrm{~mL} / \mathrm{min}, \mathrm{t}_{\mathrm{R}}(R, R)=9.34 \mathrm{~min}\right)$.

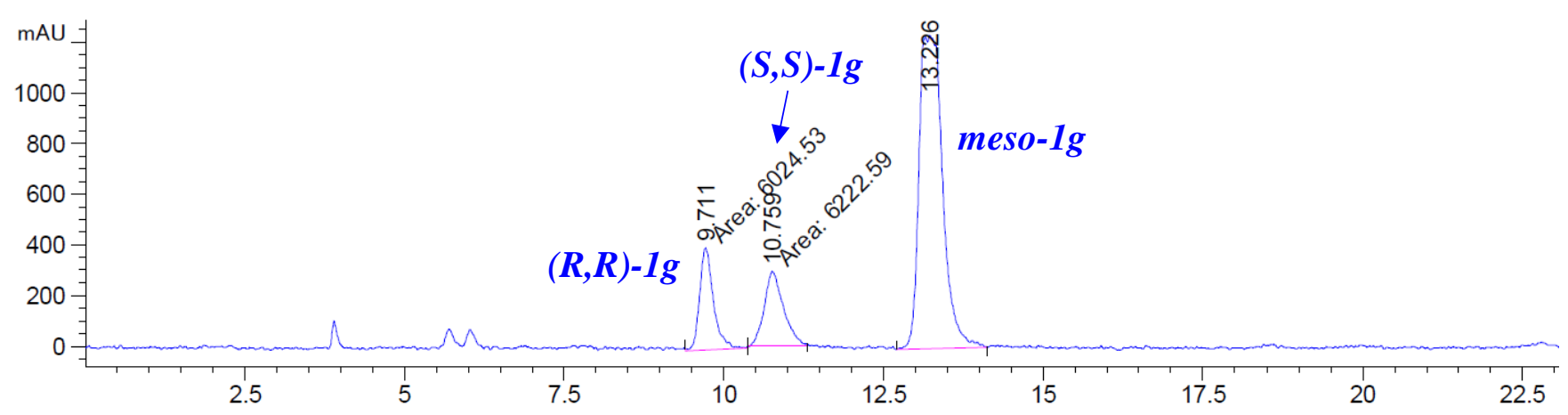




\begin{tabular}{|c|c|c|c|c|c|c|}
\hline $\begin{array}{l}\text { eak } \\
\text { \# }\end{array}$ & $\begin{array}{c}\text { RetTime } \\
\text { [min] }\end{array}$ & Type & $\begin{array}{l}\text { Width } \\
\text { [min] }\end{array}$ & $\begin{array}{c}\text { Area } \\
{\left[\mathrm{mAU}^{*} \mathrm{~s}\right]}\end{array}$ & $\begin{array}{l}\text { Height } \\
{[\mathrm{mAU}]}\end{array}$ & $\begin{array}{c}\text { Area } \\
\quad \%\end{array}$ \\
\hline \multicolumn{7}{|c|}{$---|-------|----|-------|----------|----------|--------$} \\
\hline 1 & & MM & & & & \\
\hline 2 & & MM & & 622 & & \\
\hline 3 & 13.226 & $\mathrm{BB}$ & 0.3219 & $3.17628 e 4$ & 1235.54028 & 72.1719 \\
\hline
\end{tabular}

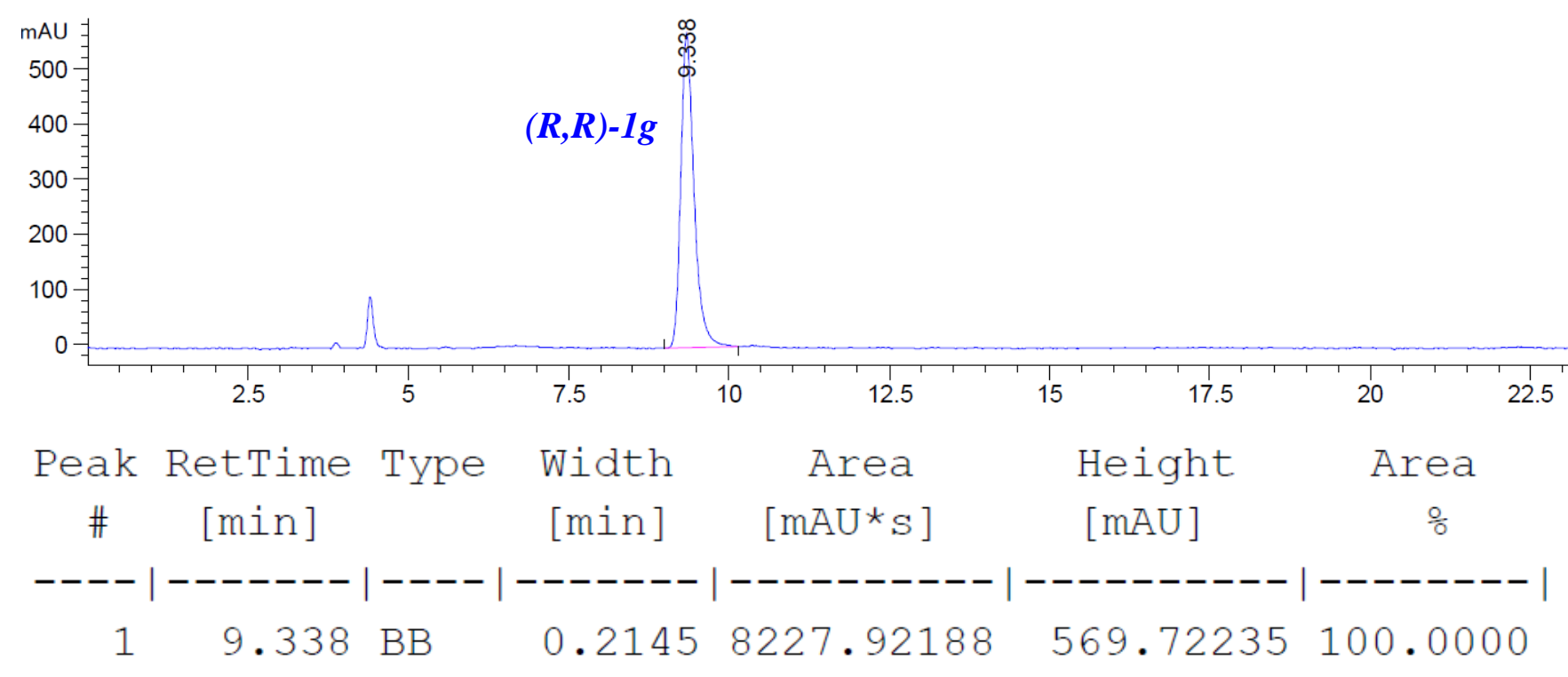

\section{2,6-Bis((R)-1-(3,5-dimethylphenyl)ethyl)-4-methylaniline 1h}

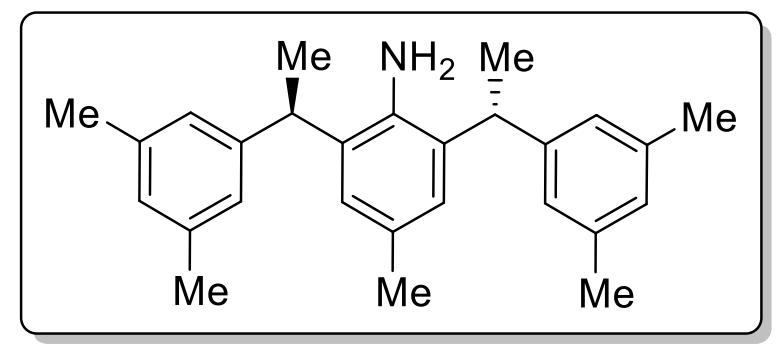

Prepared according to a reported procedure, spectroscopic data matched those previously reported in the literature. $^{5}$

${ }^{1}$ H NMR (600 MHz, Chloroform-d): $\delta 7.06$ (s, 2H), $6.80(\mathrm{~s}, 2 \mathrm{H}), 6.76$ (s, 4H), 3.95 (q, J = 7.1 Hz, 2H), 2.39 (s, 3H), 2.23 (s, 12H), 1.57 (d, $J=7.1 \mathrm{~Hz}, 6 \mathrm{H})$.

${ }^{13}$ C NMR (151 MHz, Chloroform-d): $\delta$ 145.3, 138.3, 128.4, 126.7, 125.4, 40.4, 22.5, 21.5, 21.4.

e.r.: >99:1 (Chiralpak IF, Hexane/IPA $=99.5 / 0.5, \lambda=210 \mathrm{~nm}, 1.00 \mathrm{~mL} / \mathrm{min}, \mathrm{t}_{\mathrm{R}}(R, R)=4.48 \mathrm{~min}$ ). 


\section{SUPPORTING INFORMATION}
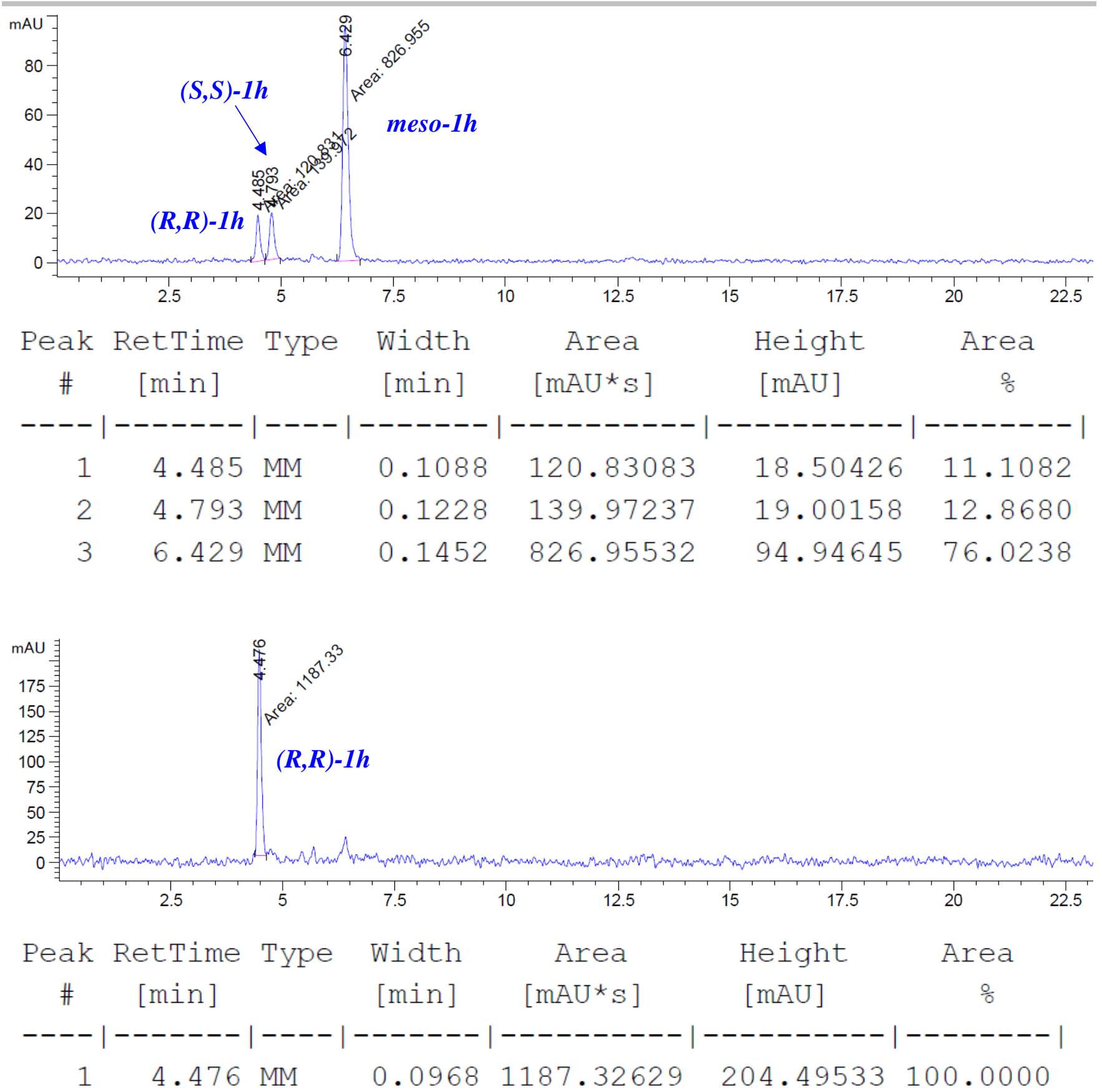

\section{2,6-Bis((R)-1-(3,5-difluorophenyl)ethyl)-4-methylaniline 1i}

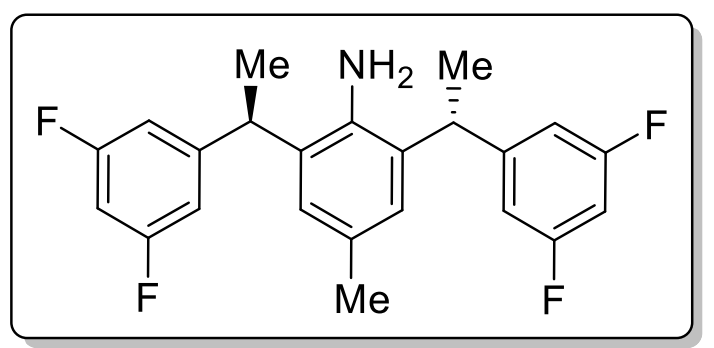

Prepared according to $\underline{\text { GP1. }}$. 


\section{Yield: $87 \%$}

\section{Appearance: colourless oil}

${ }^{1}$ H NMR (400 MHz, Chloroform-d): $\delta 7.01$ (s, 2H), 6.65 (m, 6H), 3.99 (q, J = 7.1 Hz, 2H), 3.17 (bs, 2H), $2.36(\mathrm{~s}, 3 \mathrm{H}), 1.59(\mathrm{~d}, J=7.1 \mathrm{~Hz}, 6 \mathrm{H})$.

${ }^{13}$ C NMR (101 MHz, Chloroform-d): $\delta 163.37(\mathrm{dd}, J=248.7,12.9 \mathrm{~Hz}), 150.25(\mathrm{t}, J=8.2 \mathrm{~Hz}), 139.33$, 128.98, 127.65, 126.60, $110.39(\mathrm{dd}, J=18.2,6.1 \mathrm{~Hz}), 102.02(\mathrm{t}, J=25.4 \mathrm{~Hz}), 40.24(\mathrm{t}, J=2.0 \mathrm{~Hz}), 22.05$, 21.25 .

${ }^{19}$ F NMR (376 MHz, Chloroform-d): $\delta$-109.57, -109.56.

IR (ATR): $v\left(\mathrm{~cm}^{-1}\right)$ 3471, 3391, 2971, 2936, 2876, 1620, 1593, 1449, 1311, 1115, 978, 855.

HRMS (ESI/QTOF): calcd for $\mathrm{C}_{23} \mathrm{H}_{22} \mathrm{~F}_{4} \mathrm{~N}^{+}[\mathrm{M}+\mathrm{H}]^{+}: 388.1683$; found 388.1676.

$\boldsymbol{\alpha}_{\mathrm{D}}:+0.67\left(c=1.0, \mathrm{CHCl}_{3}\right)$

e.r.: $>99: 1$ (Chiralpak IB, Hexane/IPA $\left.=99.95 / 0.05, \lambda=254 \mathrm{~nm}, 1.00 \mathrm{~mL} / \mathrm{min}, \mathrm{t}_{\mathrm{R}}(R, R)=14.5 \mathrm{~min}\right)$.

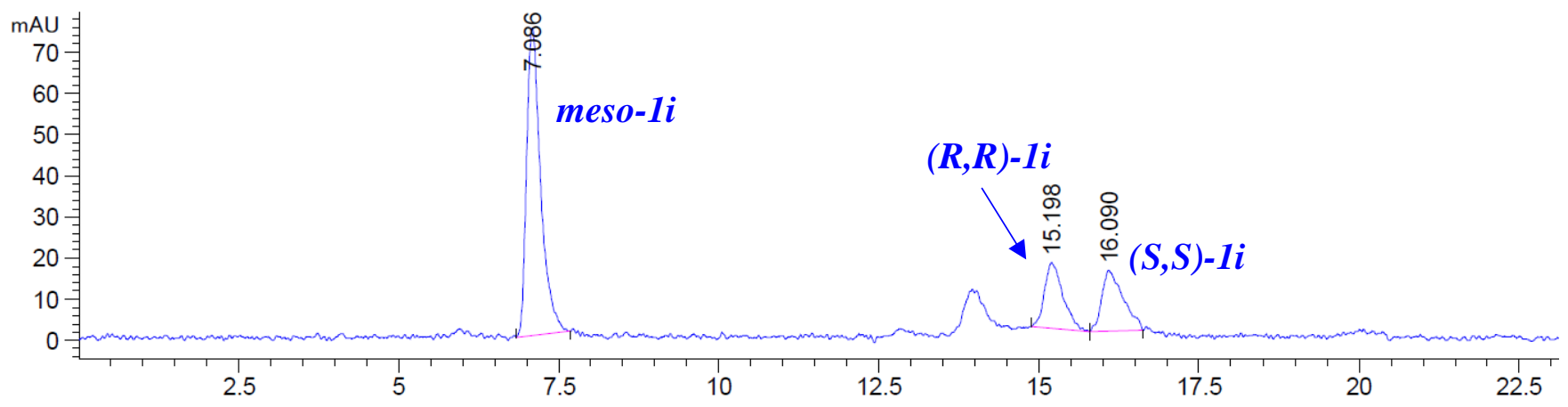

Peak RetTime Type width Area Height Area

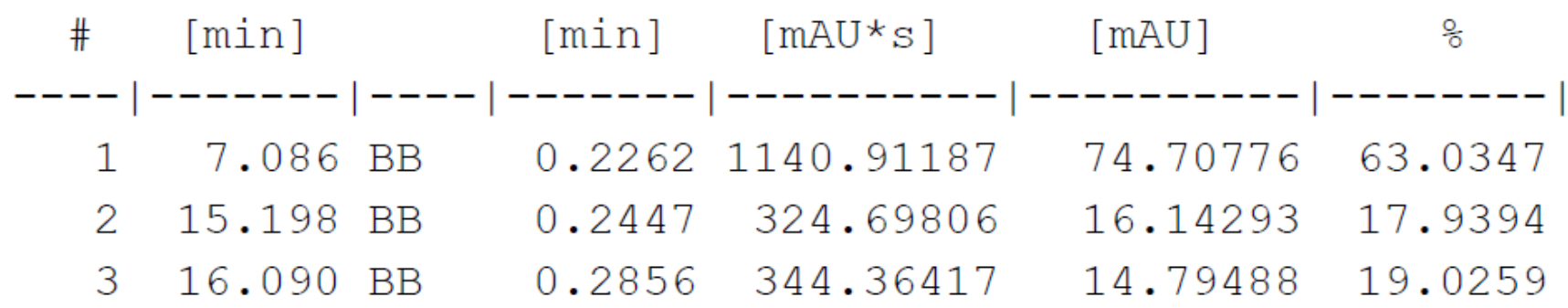

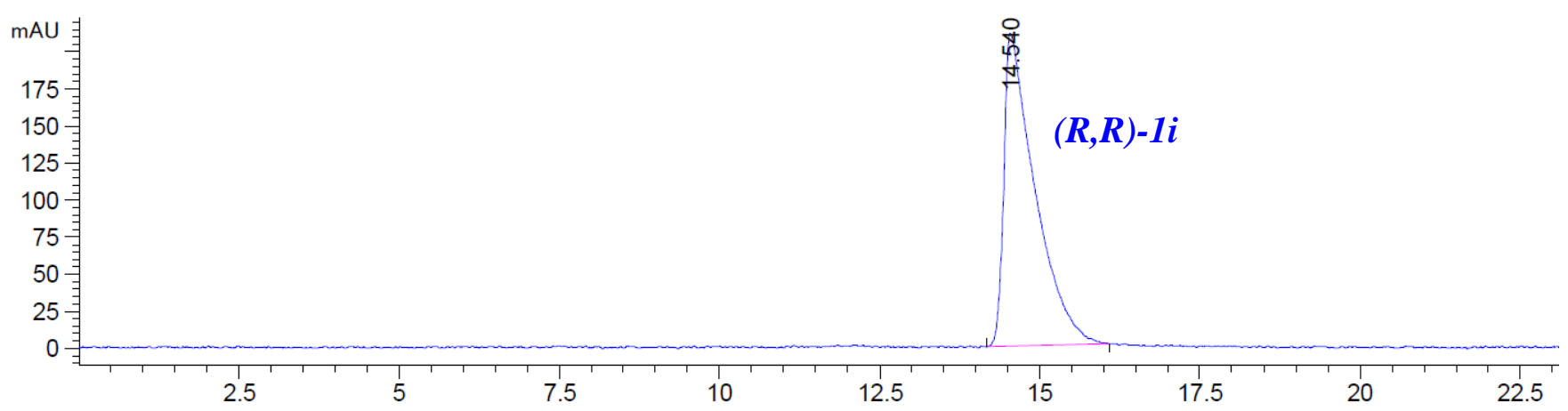


WILEY-VCH

SUPPORTING INFORMATION

\begin{tabular}{|c|c|c|c|c|c|c|}
\hline $\begin{array}{c}\text { Peak } \\
\#\end{array}$ & $\begin{array}{c}\text { RetTime } \\
\text { [min] }\end{array}$ & Type & $\begin{array}{l}\text { Width } \\
\text { [min] }\end{array}$ & $\begin{array}{c}\text { Area } \\
{\left[\mathrm{mAU}^{*} \mathrm{~s}\right]}\end{array}$ & $\begin{array}{l}\text { Height } \\
\text { [mAU] }\end{array}$ & $\begin{array}{c}\text { Area } \\
\quad \%\end{array}$ \\
\hline & ----- & & 列 & $----------\mid$ & ----------- & ------- \\
\hline 1 & 14.540 & BB & .4487 & 7141.08252 & 211.09944 & 100.0000 \\
\hline
\end{tabular}




\section{Synthesis and characterization of chiral ligands L1-9}

\section{General procedure for the synthesis of chiral ligands L1-9 (GP2)}

Chiral ligands L1-9 were synhtesized via imine condensation (Scheme 3) according to the synthetic procedure reported below.

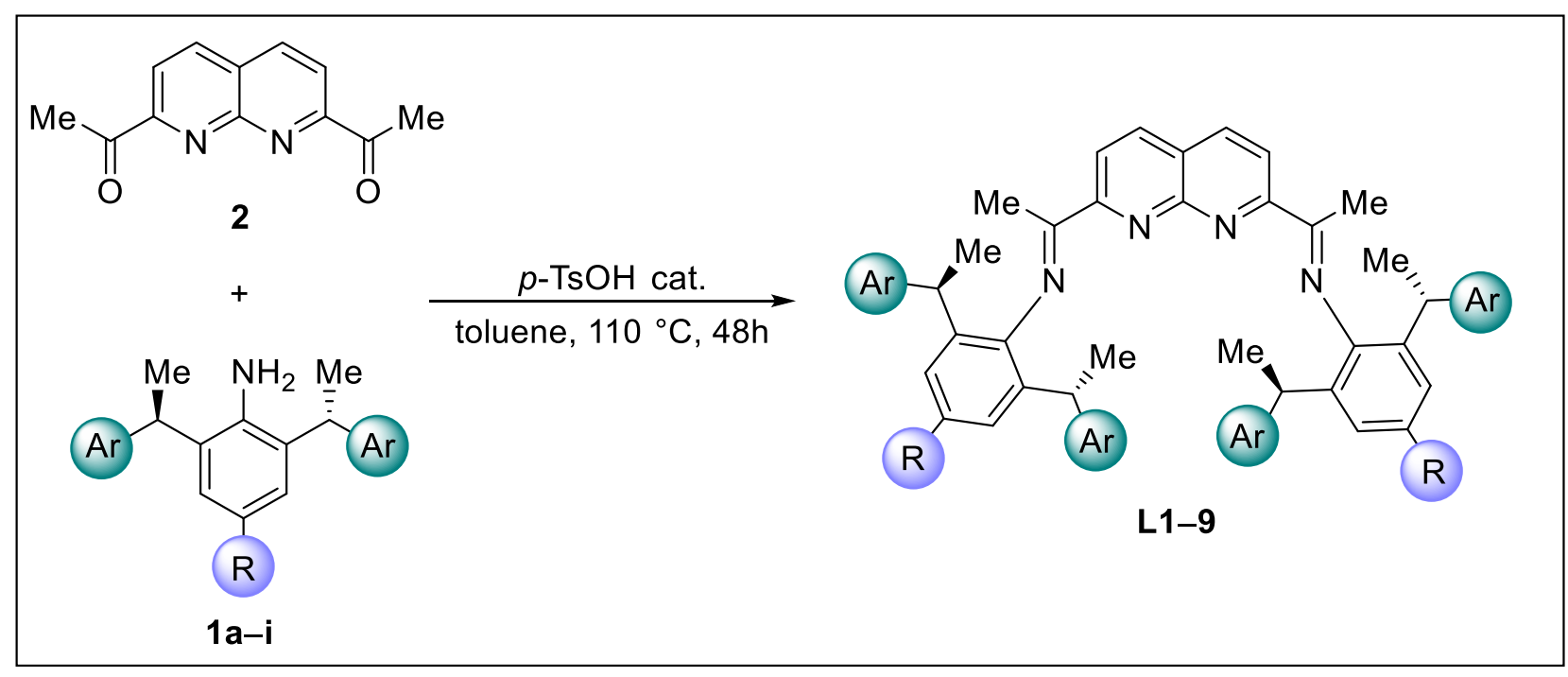

Scheme 3: Synthetic procedure to access chiral ligands L1-9.

To a solution of 1,1'-(1,8-naphthyridine-2,7-diyl)diethanone 2 (1.00 equiv.) in toluene $(0.20 \mathrm{M})$, were added aniline 1a-i (2.10 equiv.) and $p$-toluenesulfonic acid monohydrate (5.00 mol\%). The mixture was refluxed for 48 hours, then concentrated under reduced pressure to afford a mixture of ligand and monocondensation adduct. The crude mixture was purified by silica gel column chromatography (phosphate buffered silica). Recrystallization from hot EtOH afforded products L1-9 as yellow solids. Ligands L19 were dried under high vacuum for $16 \mathrm{~h}$ before being stored in the glovebox at $-30{ }^{\circ} \mathrm{C}$.

Note: The mono-condensation adducts can be isolated by column chromatography and resubmitted to the reaction conditions. 


\section{$\underline{\left(R, R, N, N^{\prime} E, N, N^{\prime} E\right)-N, N^{\prime}-((1,8-N a p h t h y r i d i n e-2,7-d i v l) b i s(e t h a n-1-y l-1-y l i d e n e)) b i s(4-}$} methyl-2,6-bis((R)-1-phenylethyl)aniline) L1

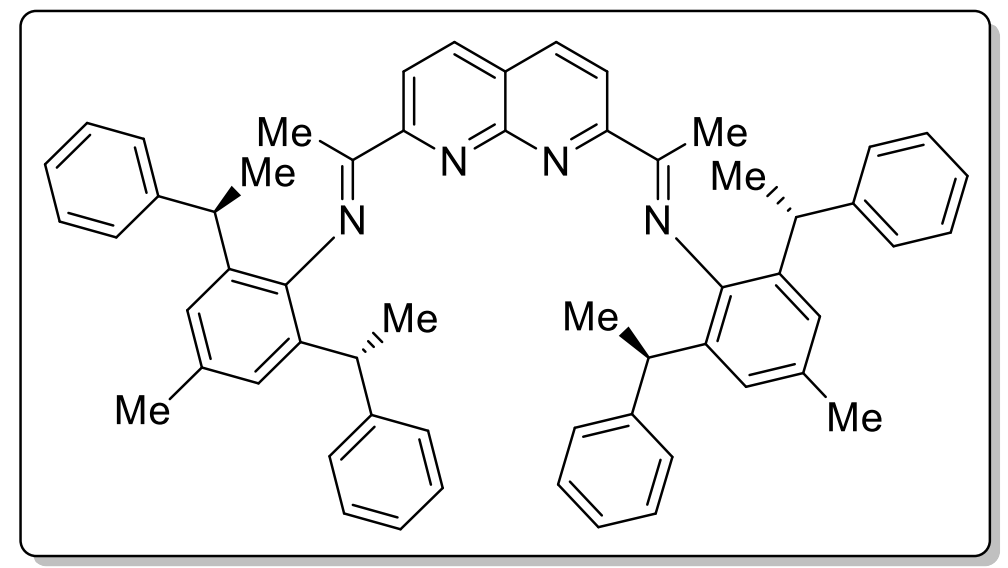

Prepared according to $\underline{\text { GP2 }}$.

Yield: $74 \%$

Appearance: yellow solid

${ }^{1}$ H NMR (400 MHz, Chloroform- $d$ ): $\delta 8.56(\mathrm{~d}, J=8.4 \mathrm{~Hz}, 2 \mathrm{H}), 8.31(\mathrm{~d}, J=8.4 \mathrm{~Hz}, 2 \mathrm{H}), 7.21-6.94$ $(\mathrm{m}, 24 \mathrm{H}), 4.02(\mathrm{q}, J=7.2 \mathrm{~Hz}, 2 \mathrm{H}), 3.85(\mathrm{q}, J=7.2 \mathrm{~Hz}, 2 \mathrm{H}), 2.41(\mathrm{~s}, 6 \mathrm{H}), 1.78(\mathrm{~s}, 6 \mathrm{H}), 1.54(\mathrm{~d}, J=7.2$ $\mathrm{Hz}, 12 \mathrm{H})$.

${ }^{13}$ C NMR (101 MHz, Chloroform-d): $\delta$ 168.89, 159.53, 155.07, 146.52, 146.32, 145.16, 136.96, 133.27, $132.66,132.57,128.44,128.19,127.90,127.48,125.92$, 125.81, 125.76, 125.70, 124.24, 120.79, 40.89, $38.72,22.70,21.63,21.19,17.13$.

IR (ATR): $v\left(\mathrm{~cm}^{-1}\right)$ 3059, 3024, 2966, 2930, 2872, 1641, 1598, 1492, 1477, 1314, 1094, 906, 863, 728, 672 .

HRMS (ESI/QTOF): calcd for $\mathrm{C}_{58} \mathrm{H}_{57} \mathrm{~N}_{4}{ }^{+}[\mathrm{M}+\mathrm{H}]^{+}$: 809.4578; found: 809.4583 .

$[\boldsymbol{\alpha}] \mathbf{D}^{\mathbf{2 0}}=+28.3\left(c=1.0, \mathrm{CHCl}_{3}\right)$.

Melting point: $204-207^{\circ} \mathrm{C}$ 


\section{$\left(R, R, N, N^{\prime} E, N, N^{\prime} E\right)-N, N^{\prime}-((1,8-N a p h t h y r i d i n e-2,7-d i v l) b i s($ ethan-1-yl-1-ylidene $))$ bis(2,6-}

\section{bis((R)-1-phenylethyl)aniline) L2}

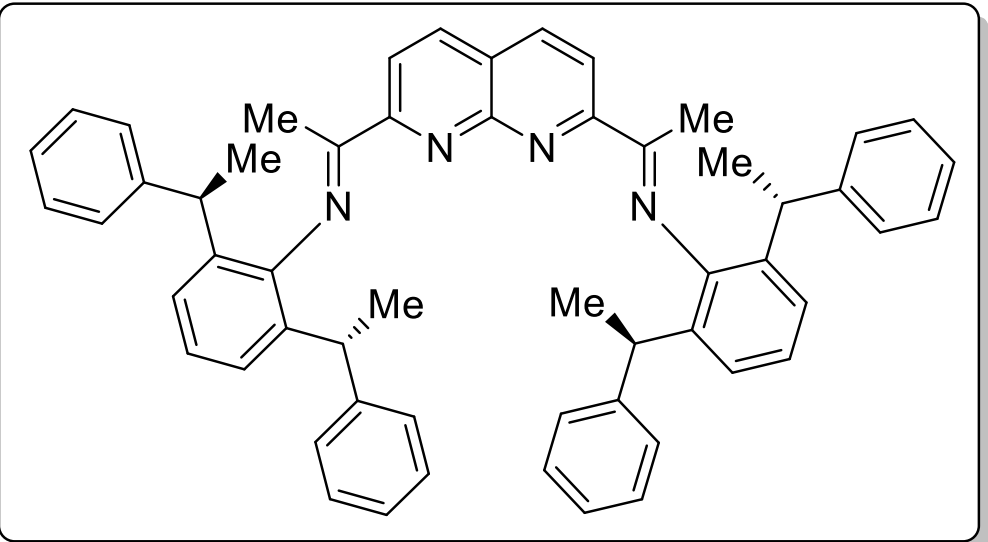

Prepared according to $\underline{\mathbf{G P 2}}$.

Yield: $74 \%$

Appearance: yellow solid

${ }^{1}$ H NMR (400 MHz, Chloroform-d): $\delta 8.56(\mathrm{~d}, J=8.5 \mathrm{~Hz}, 2 \mathrm{H}), 8.33(\mathrm{~d}, J=8.5 \mathrm{~Hz}, 2 \mathrm{H}), 7.36$ (ddd, $J=$ 7.8, 6.2, $1.3 \mathrm{~Hz}, 4 \mathrm{H}), 7.23-6.97(\mathrm{~m}, 22 \mathrm{H}), 4.04$ (q, $J=7.2 \mathrm{~Hz}, 2 \mathrm{H}), 3.88(\mathrm{q}, J=7.2 \mathrm{~Hz}, 2 \mathrm{H}), 1.79$ (s, $6 \mathrm{H}), 1.56(\mathrm{~d}, J=7.2 \mathrm{~Hz}, 12 \mathrm{H})$.

${ }^{13}$ C NMR (101 MHz, Chloroform-d): $\delta$ 168.62, 159.41, 155.06, 147.60, 146.44, 146.19, 137.02, 133.37, 132.83 , 128.47, 128.20, 127.89, 127.49, 125.98, 125.81, 125.20, 125.00, 124.28, 123.52, 120.81, 40.98, $38.93,22.69,21.31,17.19$.

IR (ATR): $v\left(\mathrm{~cm}^{-1}\right)$ 3060, 3024, 2966, 2930, 2872, 1640, 1599, 1492, 1365, 1094, 907, 729, 698.

HRMS (ESI/QTOF): calcd for $\mathrm{C}_{56} \mathrm{H}_{53} \mathrm{~N}_{4}{ }^{+}[\mathrm{M}+\mathrm{H}]^{+}:$: 781.4265; found: 781.4270 .

$[\boldsymbol{\alpha}] \mathbf{D}^{\mathbf{2 0}}=+120\left(c=1.0, \mathrm{CHCl}_{3}\right)$.

Melting point: $114-117^{\circ} \mathrm{C}$ 


\section{$\underline{\left(R, R, N, N^{\prime} E, N, N^{\prime} E\right)-N, N^{\prime}-((1,8-N a p h t h y r i d i n e-2,7-d i v l) b i s(e t h a n-1-y l-1-y l i d e n e)) b i s(4-}$}

\section{methoxy-2,6-bis((R)-1-phenylethyl)aniline) L3}

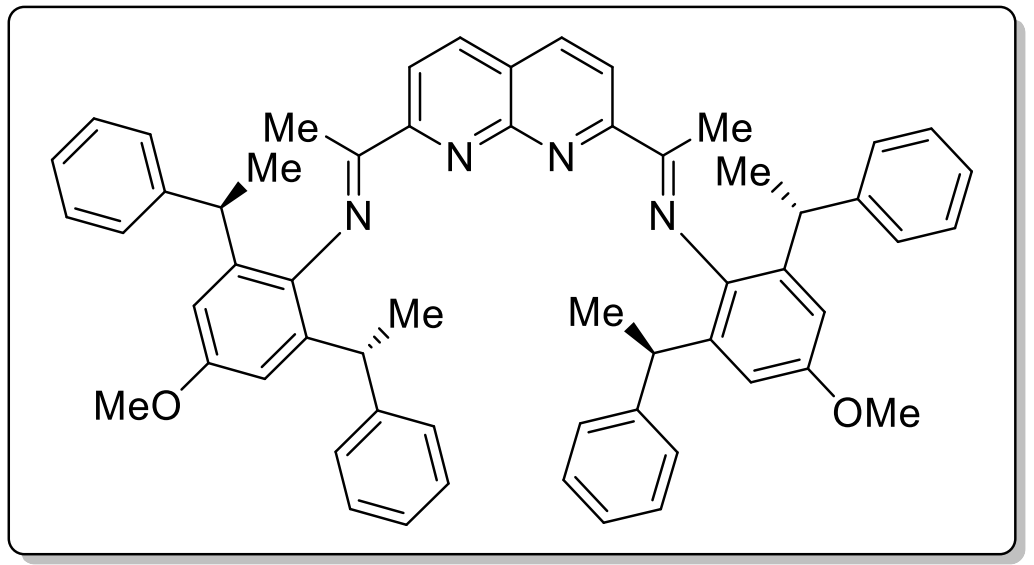

Prepared according to $\underline{\text { GP2 }}$.

Yield: $65 \%$

Appearance: yellow solid

${ }^{1}$ H NMR (400 MHz, Chloroform- $d$ ): $\delta 8.58(\mathrm{~d}, J=8.4 \mathrm{~Hz}, 2 \mathrm{H}), 8.32(\mathrm{~d}, J=8.4 \mathrm{~Hz}, 2 \mathrm{H}), 7.21-6.97$ $(\mathrm{m}, 20 \mathrm{H}), 6.96-6.89(\mathrm{~m}, 4 \mathrm{H}), 4.05(\mathrm{q}, J=7.2 \mathrm{~Hz}, 2 \mathrm{H}), 3.87(\mathrm{~m}, 8 \mathrm{H}), 1.80(\mathrm{~s}, 6 \mathrm{H}), 1.55$ (d, J = $7.3 \mathrm{~Hz}$, $12 \mathrm{H})$.

${ }^{13}$ C NMR (101 MHz, Chloroform-d): $\delta$ 169.56, 159.56, 156.12, 155.05, 146.15, 145.94, 141.27, 136.96, $134.81,134.07,128.46,128.22$, 127.84, 127.44, 126.02, 125.87, 124.25, 120.75, 110.91, 110.54, 55.57, 41.04, 38.95, 22.58, 21.14, 17.08 .

IR (ATR): $v\left(\mathrm{~cm}^{-1}\right)$ 3026, 2968, 2934, 2873, 2248, 1639, 1599, 1437, 1235, 1147, 1039, 905, 726, 698.

HRMS (ESI/QTOF): calcd for $\mathrm{C}_{58} \mathrm{H}_{57} \mathrm{~N}_{4} \mathrm{O}_{2}{ }^{+}[\mathrm{M}+\mathrm{H}]^{+}$: 842.4510; found: 842.4514.

$[\boldsymbol{\alpha}] \mathbf{D}^{\mathbf{2 0}}=-86.3\left(c=1.0, \mathrm{CHCl}_{3}\right)$.

Melting point: $97.3-100{ }^{\circ} \mathrm{C}$ 


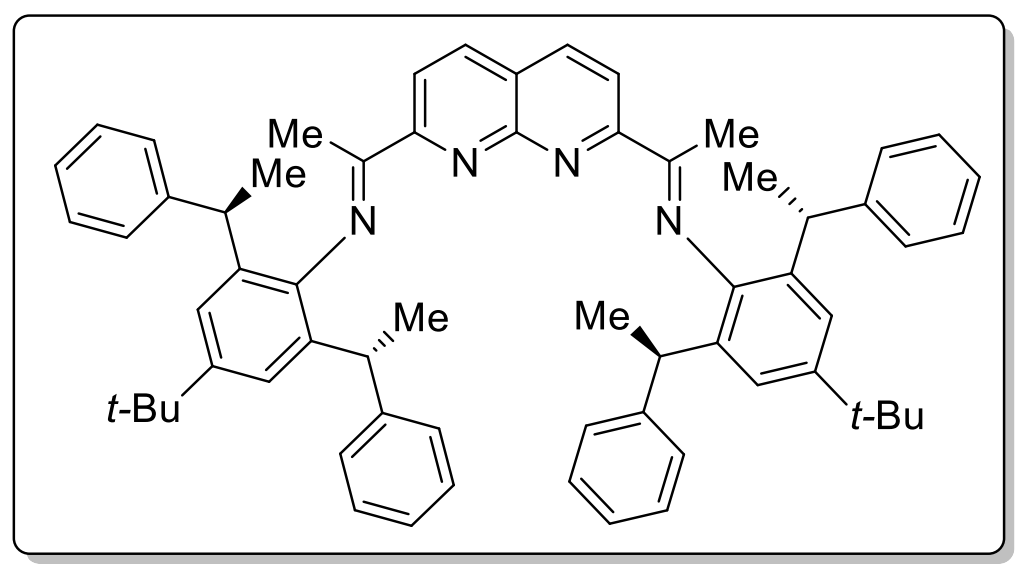

Prepared according to $\underline{\mathbf{G P 2}}$.

Yield: $61 \%$

Appearance: yellow solid

${ }^{1}$ H NMR (400 MHz, Chloroform- $\left.d\right): \delta 8.55(\mathrm{~d}, J=8.5 \mathrm{~Hz}, 2 \mathrm{H}), 8.31(\mathrm{~d}, J=8.5 \mathrm{~Hz}, 2 \mathrm{H}), 7.39(\mathrm{~d}, J=$ $2.0 \mathrm{~Hz}, 2 \mathrm{H}), 7.37$ (d, $J=2.0 \mathrm{~Hz}, 2 \mathrm{H}), 7.20-6.94(\mathrm{~m}, 20 \mathrm{H}), 4.03(\mathrm{q}, J=7.2 \mathrm{~Hz}, 2 \mathrm{H}), 3.85$ (q, $J=7.2 \mathrm{~Hz}$, $2 \mathrm{H}), 1.74(\mathrm{~s}, 6 \mathrm{H}), 1.56(\mathrm{dd}, J=7.2,1.9 \mathrm{~Hz}, 12 \mathrm{H}), 1.40$ (s, 18H).

${ }^{13}$ C NMR (101 MHz, Chloroform-d): $\delta$ 168.70, 159.52, 155.09, 146.80, 146.40, 145.80, 145.12, 136.93, 132.43, 131.76, 128.41, 128.15, 127.86, 127.51, 125.86, 125.69, 124.21, 121.87, 121.83, 120.77, 41.18, $39.17,34.78,31.84,29.86,24.74,22.88,21.45,17.20$.

IR (ATR): $v\left(\mathrm{~cm}^{-1}\right)$ 3025, 2963, 2932, 2905, 2871, 1645, 1599, 1492, 1449, 1364, 1095, 909, 760, 733, 699.

HRMS (ESI/QTOF): calcd for $\mathrm{C}_{64} \mathrm{H}_{69} \mathrm{~N}_{4}{ }^{+}[\mathrm{M}+\mathrm{H}]^{+}$: 893.5517; found: 893.5522 .

$[\boldsymbol{\alpha}] \mathbf{D}^{\mathbf{2 0}}=-21.8\left(c=1.0, \mathrm{CHCl}_{3}\right)$.

Melting point: $120-123^{\circ} \mathrm{C}$ 


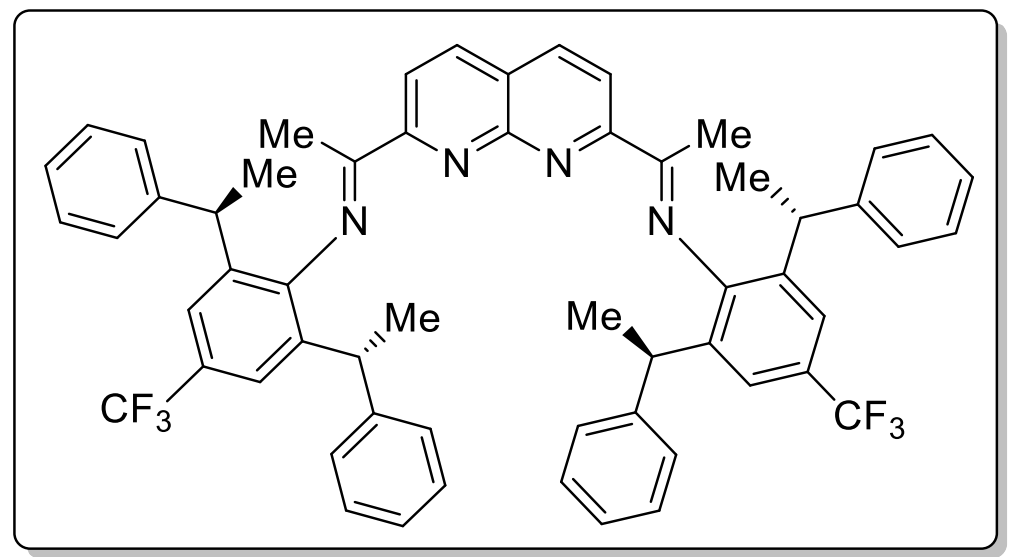

Prepared according to $\underline{\text { GP2 }}$.

Yield: $56 \%$

Appearance: yellow solid

${ }^{1} \mathrm{H}$ NMR (400 MHz, Chloroform- $\left.\boldsymbol{d}\right): \delta 8.48(\mathrm{~d}, J=8.4 \mathrm{~Hz}, 2 \mathrm{H}), 8.35(\mathrm{~d}, J=8.5 \mathrm{~Hz}, 2 \mathrm{H}), 7.62(\mathrm{~d}, J=$ $5.9 \mathrm{~Hz}, 4 \mathrm{H}), 7.21-6.87(\mathrm{~m}, 20 \mathrm{H}), 4.03(\mathrm{q}, J=7.3 \mathrm{~Hz}, 2 \mathrm{H}), 3.87$ (q, $J=7.3 \mathrm{~Hz}, 2 \mathrm{H}), 1.72(\mathrm{~d}, J=2.1 \mathrm{~Hz}$, $6 \mathrm{H}), 1.58(\mathrm{~d}, J=7.3 \mathrm{~Hz}, 12 \mathrm{H})$.

${ }^{13}$ C NMR (101 MHz, Chloroform- $d$ ): $\delta$ 168.95, 158.93, 154.97, 150.58, 145.47, 145.12, 137.22, 134.13, 133.47, 128.70, 128.37, 127.81, 127.41, 126.37, 126.18, 125.78 (q, $J=32.3 \mathrm{~Hz}), 124.47,122.25$ (q, $J=$ $4.04 \mathrm{~Hz}), 122.02$ (q, $J=3.03 \mathrm{~Hz}), 120.88,41.10,39.18,22.66,21.24,17.42$.

${ }^{19}$ F NMR (376 MHz, Chloroform- $d$ ): $\delta-61.39$.

IR (ATR): $v\left(\mathrm{~cm}^{-1}\right)$ 3026, 2968, 2933, 1647, 1599, 1349, 1292, 1180, 1158, 1117, 733, 699.

HRMS (ESI/QTOF): calcd for $\mathrm{C}_{58} \mathrm{H}_{51} \mathrm{~F}_{6} \mathrm{~N}_{4}{ }^{+}[\mathrm{M}+\mathrm{H}]^{+}$: 917.4013; found: 917.4018.

$[\boldsymbol{\alpha}] \mathbf{D}^{20}=+220\left(c=1.0, \mathrm{CHCl}_{3}\right)$.

Melting point: $121-125^{\circ} \mathrm{C}$ 


\section{butyl)phenyl)ethyl)-4-methylaniline L6}

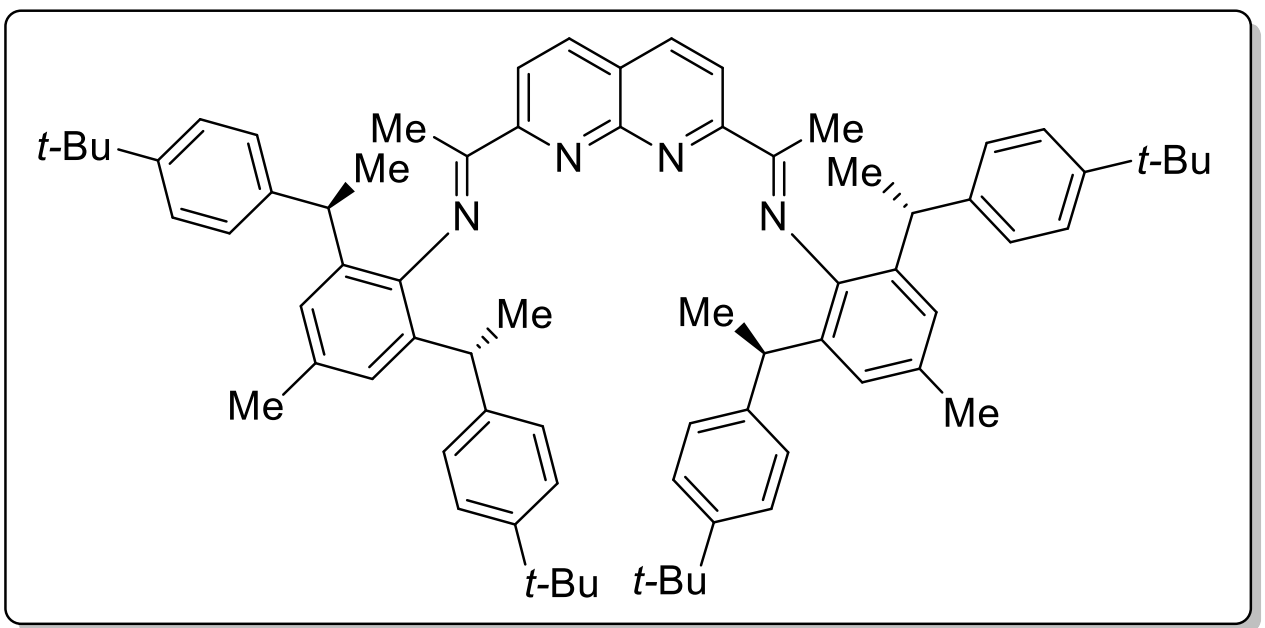

Prepared according to $\underline{\mathbf{G P 2}}$.

Yield: $69 \%$

Appearance: yellow solid

${ }^{1}$ H NMR (400 MHz, Chloroform- $d$ ): $\delta 8.50(\mathrm{~d}, J=8.5 \mathrm{~Hz}, 2 \mathrm{H}), 8.29(\mathrm{~d}, J=8.5 \mathrm{~Hz}, 2 \mathrm{H}), 7.21-7.17$ (m, 4H), $7.14-7.07(\mathrm{~m}, 8 \mathrm{H}), 7.03(\mathrm{~d}, J=8.2 \mathrm{~Hz}, 4 \mathrm{H}), 6.93(\mathrm{~d}, J=8.0 \mathrm{~Hz}, 4 \mathrm{H}), 3.98(\mathrm{q}, J=7.1 \mathrm{~Hz}, 2 \mathrm{H})$, $3.85(\mathrm{q}, J=7.2 \mathrm{~Hz}, 2 \mathrm{H}), 2.39(\mathrm{~s}, 6 \mathrm{H}), 1.81(\mathrm{~s}, 6 \mathrm{H}), 1.54(\mathrm{~d}, J=7.2 \mathrm{~Hz}, 12 \mathrm{H}), 1.27(\mathrm{~s}, 18 \mathrm{H}), 1.22(\mathrm{~s}, 18 \mathrm{H})$. ${ }^{13}$ C NMR (101 MHz, Chloroform-d): $\delta$ 169.06, 159.54, 154.98, 148.51, 148.29, 145.13, 143.27, 136.79, 133.66, 132.90, 132.51, 127.58, 127.01, 126.70, 125.86, 125.66, 125.28, 125.10, 124.14, 120.74, 40.17, $38.20,34.41,31.55,31.47,29.86,22.53,21.62,21.15,17.17$.

IR (ATR): $v\left(\mathrm{~cm}^{-1}\right)$ 2961, 2904, 2869, 1640, 1598, 1507, 1460, 1364, 1095, 1011, 833, 733.

HRMS (ESI/QTOF): calcd for $\mathrm{C}_{74} \mathrm{H}_{89} \mathrm{~N}_{4}{ }^{+}[\mathrm{M}+\mathrm{H}]^{+}$: 1033.7082 ; found: 1033.7087 .

$[\boldsymbol{\alpha}] \mathbf{D}^{\mathbf{2 0}}=+106\left(c=1.0, \mathrm{CHCl}_{3}\right)$.

Melting point: $130-133^{\circ} \mathrm{C}$ 


\section{$\underline{\left(R, R, N, N^{\prime} E, N, N^{\prime} E\right)-N, N^{\prime}-((1,8-N a p h t h y r i d i n e-2,7-d i v l) b i s(e t h a n-1-y l-1-y l i d e n e)) b i s(4-}$} methyl-2,6-bis((R)-1-(naphthalen-2-yl)ethyl)aniline) L7

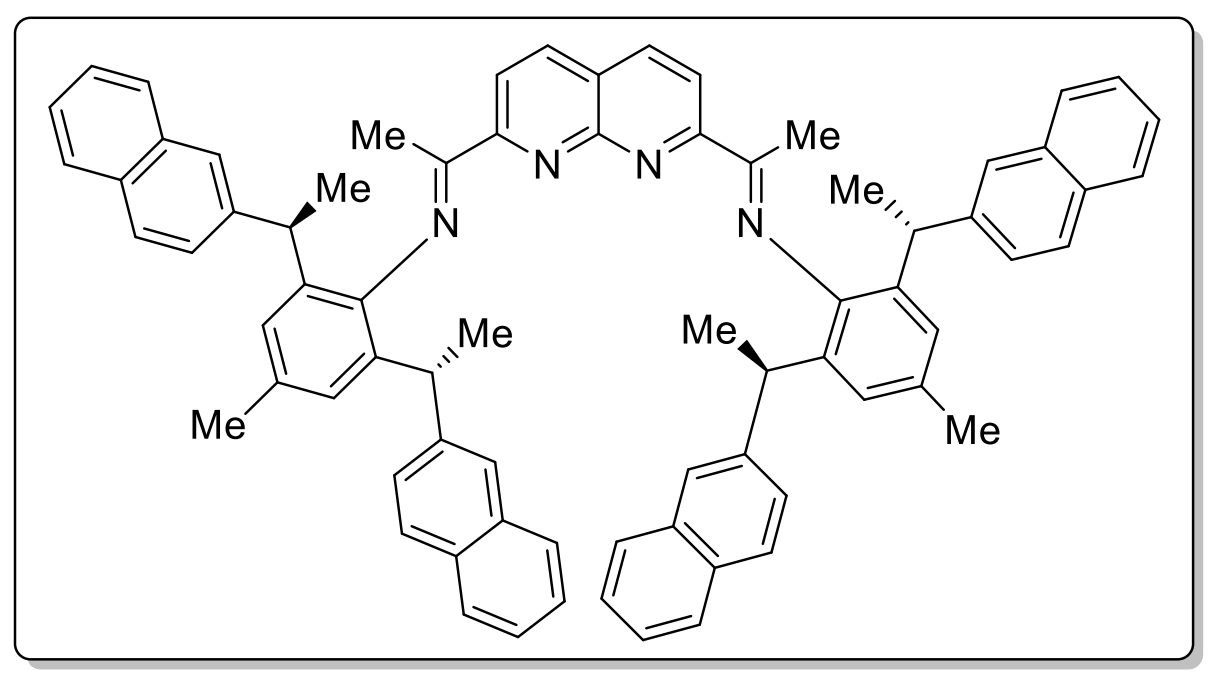

Prepared according to $\underline{\mathbf{G P 2}}$.

Yield: $60 \%$

Appearance: yellow solid

${ }^{1}$ H NMR (400 MHz, Chloroform-d): $\delta 8.48$ (d, $\left.J=8.3 \mathrm{~Hz}, 2 \mathrm{H}\right), 8.23$ (d, $\left.J=8.5 \mathrm{~Hz}, 2 \mathrm{H}\right), 7.79-7.72$ $(\mathrm{m}, 2 \mathrm{H}), 7.67(\mathrm{t}, J=8.6 \mathrm{~Hz}, 4 \mathrm{H}), 7.61-7.52(\mathrm{~m}, 4 \mathrm{H}), 7.52-7.47(\mathrm{~m}, 2 \mathrm{H}), 7.46(\mathrm{~d}, J=2.1 \mathrm{~Hz}, 4 \mathrm{H}), 7.39$ -7.27 (m, 8H), $7.24-7.13(\mathrm{~m}, 8 \mathrm{H}), 4.18(\mathrm{q}, J=7.1 \mathrm{~Hz}, 2 \mathrm{H}), 4.02(\mathrm{q}, J=7.1 \mathrm{~Hz}, 2 \mathrm{H}), 2.42(\mathrm{~s}, 6 \mathrm{H}), 1.81$ (s, 6H), $1.62(\mathrm{dd}, J=11.5,7.2 \mathrm{~Hz}, 12 \mathrm{H})$.

${ }^{13}$ C NMR (101 MHz, Chloroform-d): $\delta$ 168.85, 159.54, 154.92, 145.33, 143.81, 143.76, 136.88, 133.58, $133.51,133.29,132.71,132.50,132.09,131.99,128.01,127.78,127.70,127.67,127.63,127.57,126.88$, $126.10,126.05,125.96,125.87,125.71,125.31,125.24,125.19,124.20,120.83,40.92,39.21,22.27$, 21.64, 21.08, 17.46 .

IR (ATR): $v\left(\mathrm{~cm}^{-1}\right)$ 3052, 3017, 2965, 2929, 2872, 1640, 1599, 1505, 1450, 1364, 1142, 1095, 907, 856, 816, 730, 477.

HRMS (ESI/QTOF): calcd for $\mathrm{C}_{74} \mathrm{H}_{65} \mathrm{~N}_{4}{ }^{+}[\mathrm{M}+\mathrm{H}]^{+}:$1009.5204; found: 1009.5209 .

$[\boldsymbol{\alpha}] \mathbf{D}^{\mathbf{2 0}}=+337\left(c=1.0, \mathrm{CHCl}_{3}\right)$.

Melting point: $113-117^{\circ} \mathrm{C}$ 


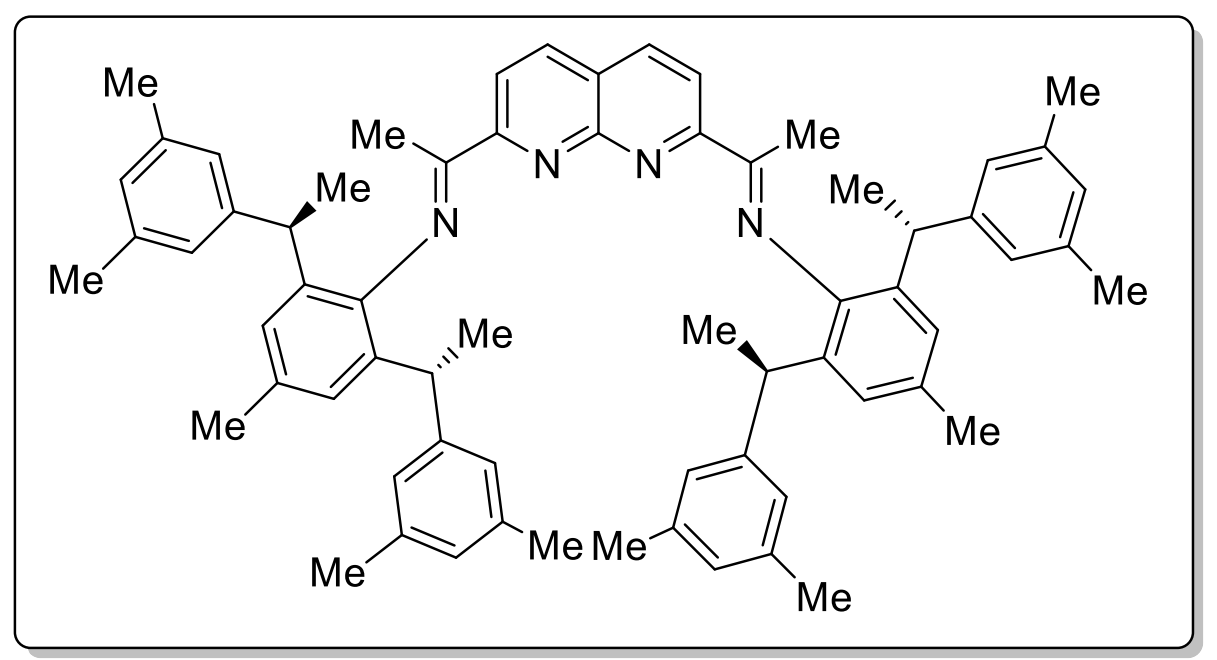

Prepared according to $\underline{\text { GP2 }}$.

Yield: $51 \%$

Appearance: yellow solid

${ }^{1} \mathrm{H}$ NMR (400 MHz, Chloroform- $d$ ): $\delta 8.56(\mathrm{~d}, J=8.5 \mathrm{~Hz}, 2 \mathrm{H}), 8.33$ (d, $\left.J=8.5 \mathrm{~Hz}, 2 \mathrm{H}\right), 7.17$ (q, $J=$ $1.8 \mathrm{~Hz}, 4 \mathrm{H}), 6.73(\mathrm{~s}, 2 \mathrm{H}), 6.64(\mathrm{~d}, J=1.6 \mathrm{~Hz}, 6 \mathrm{H}), 6.57$ (d, $J=1.6 \mathrm{~Hz}, 4 \mathrm{H}), 3.90(\mathrm{q}, J=7.1 \mathrm{~Hz}, 2 \mathrm{H}), 3.76$ (q, $J=7.2 \mathrm{~Hz}, 2 \mathrm{H}$ ), 2.46 (s, 6H), 2.13 (s, 12H), 1.99 (s, 12H), 1.65 (s, 6H), 1.53 (dd, $J=9.3,7.2 \mathrm{~Hz}, 12 \mathrm{H}$ ). ${ }^{13}$ C NMR (101 MHz, Chloroform-d): $\delta$ 168.93, 159.66, 154.99, 146.46, 146.06, 145.46, 137.69, 137.35, $136.55,133.32,132.58,132.28,127.53,127.50,125.96,125.65,125.55,125.42,123.83,120.82,40.97$, $39.21,22.57,21.71,21.43,21.23,21.19,17.05$.

IR (ATR): $v\left(\mathrm{~cm}^{-1}\right)$ 3013, 2963, 2920, 2870, 1643, 1599, 1449, 1365, 1127, 909, 847, 731.

HRMS (ESI/QTOF): calcd for $\mathrm{C}_{66} \mathrm{H}_{73} \mathrm{~N}_{4}{ }^{+}[\mathrm{M}+\mathrm{H}]^{+}:$: 921.5830; found: 921.5831 .

aD: $+77.3\left(c=1.0, \mathrm{CHCl}_{3}\right)$

Melting point: $245-248^{\circ} \mathrm{C}$ 


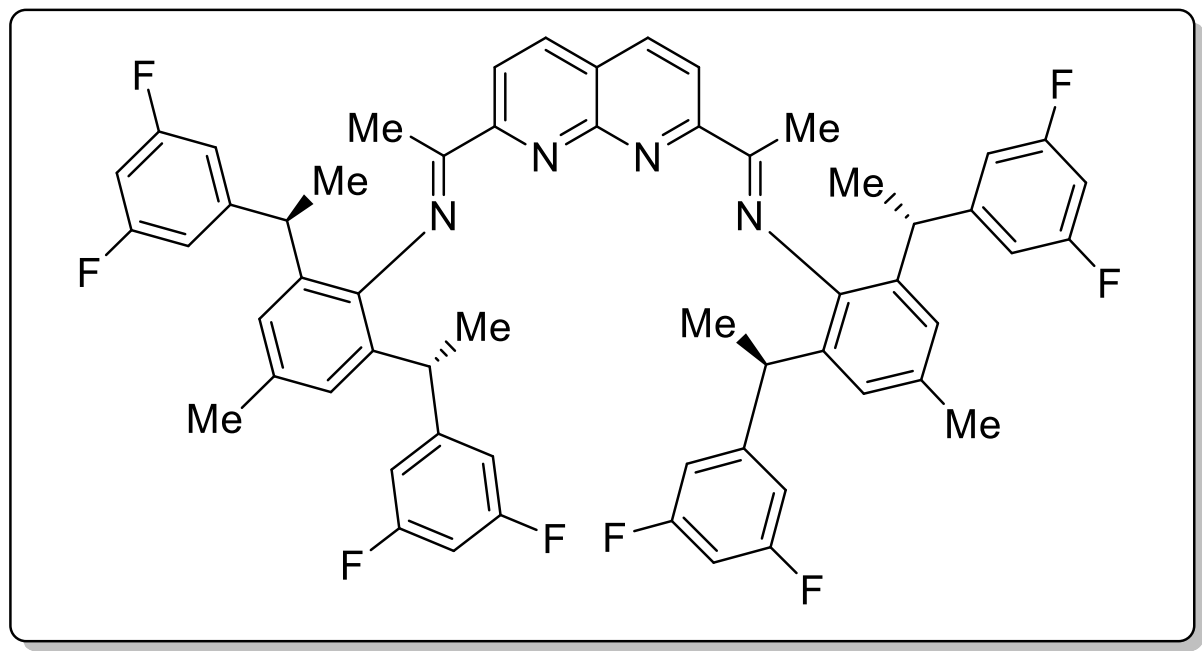

Prepared according to $\underline{\mathbf{G P 2}}$.

Yield: $67 \%$

Appearance: yellow solid

${ }^{1}$ H NMR (400 MHz, Chloroform-d): $\delta 8.47$ (d, $\left.J=8.4 \mathrm{~Hz}, 2 \mathrm{H}\right), 8.34$ (d, $\left.J=8.5 \mathrm{~Hz}, 2 \mathrm{H}\right), 7.10$ (s, 4H), $6.53(\mathrm{tq}, J=8.6,3.3,2.3 \mathrm{~Hz}, 12 \mathrm{H}), 4.00(\mathrm{q}, J=7.1 \mathrm{~Hz}, 2 \mathrm{H}), 3.80(\mathrm{q}, J=7.2 \mathrm{~Hz}, 2 \mathrm{H}), 2.42(\mathrm{~s}, 6 \mathrm{H}), 1.95$ (s, $6 \mathrm{H}), 1.52$ (dd, $J=7.2,2.3 \mathrm{~Hz}, 12 \mathrm{H})$.

${ }^{13}$ C NMR (101 MHz, Chloroform- $d$ ): $\delta$ 169.21, 163.39 (dd, $\left.J=248.9,10.1 \mathrm{~Hz}\right), 161.67(\mathrm{dd}, J=248.5$, $10.1 \mathrm{~Hz}), 159.01,155.01,150.67(\mathrm{t}, J=8.1 \mathrm{~Hz}), 150.31$ (t, $J=8.3 \mathrm{~Hz}), 145.08,137.40,133.29,132.23$, 131.57, 126.24, 126.11, 124.49, 120.43, 110.69 (dd, $J=18.2,6.1 \mathrm{~Hz}), 110.33(\mathrm{dd}, J=18.2,7.1 \mathrm{~Hz})$, $101.53(\mathrm{t}, J=26.3 \mathrm{~Hz}), 101.32(\mathrm{t}, J=25.3 \mathrm{~Hz}), 40.71,38.75,22.28,21.57,20.76,17.47$.

${ }^{19}$ F NMR (376 MHz, Chloroform-d): $\delta$-110.12, -110.83 .

IR (ATR): $v\left(\mathrm{~cm}^{-1}\right)$ 2970, 2934, 2875, 1621, 1595, 1446, 1313, 1115, 979, 854, 731.

HRMS (ESI/QTOF): calcd for $\mathrm{C}_{58} \mathrm{H}_{49} \mathrm{~F}_{8} \mathrm{~N}^{+}[\mathrm{M}+\mathrm{H}]^{+}$: 953.3824 ; found: 953.3829 . $[\alpha] \mathbf{D}^{20}=+58.5\left(c=1.0, \mathrm{CHCl}_{3}\right)$.

Melting point: $84.6-88.3{ }^{\circ} \mathrm{C}$ 


\section{Synthesis and characterization of novel 1,1-dichloroalkenes}

\section{(4-(2,2-Dichlorovinyl)phenoxy)triisopropylsilane 14}<smiles></smiles>

To a $50 \mathrm{~mL}$ dry round-bottom flask were added triphenylphosphine $\mathrm{PPh}_{3}(24.4 \mathrm{mmol}, 4.00$ eq.) and DCM $(12.2 \mathrm{~mL}, 0.50 \mathrm{M})$. The suspension was cooled to $0{ }^{\circ} \mathrm{C}$, whereby 4-((triisopropylsilyl)oxy)benzaldehyde ${ }^{8}$ ( $6.10 \mathrm{mmol}, 1.00$ eq.) and $\mathrm{CCl}_{4}(12.2 \mathrm{mmol}, 2.00$ eq.) were added sequentially. The reaction was allowed to warm to room temperature and stirred at this temperature until TLC ( $2 \% \mathrm{Et}_{2} \mathrm{O}$ in pentane) showed full conversion of starting material (approximately 8 hours). The mixture was then diluted with $\mathrm{H}_{2} \mathrm{O}$ and $\mathrm{Et}_{2} \mathrm{O}$, layers were separated, the aqueous layer was washed with $\mathrm{Et}_{2} \mathrm{O}(2 \times)$. The combined organics were dried over $\mathrm{MgSO}_{4}$, filtered, and concentrated under reduced pressure to afford the crude product. Purification via flash column chromatogtraphy $\left(\mathrm{SiO}_{2}, 2 \% \mathrm{Et}_{2} \mathrm{O}\right.$ in pentane) afforded the title compound $\mathbf{1 4}$.

Yield: $81 \%$

Appearance: colourless oil

${ }^{1}$ H NMR (400 MHz, Chloroform-d): $\delta 7.48-7.39$ (m, 2H), $6.91-6.82$ (m, 2H), 6.77 (s, 1H), $1.35-$ $1.19(\mathrm{~m}, 3 \mathrm{H}), 1.10(\mathrm{~d}, J=7.3 \mathrm{~Hz}, 18 \mathrm{H})$.

${ }^{13}$ C NMR (101 MHz, Chloroform-d): $\delta$ 156.47, 130.16, 128.30, 126.38, 120.02, 118.76, 18.04, 12.82.

IR (ATR): $v\left(\mathrm{~cm}^{-1}\right)$ 2944, 2867, 1602, 1507, 1463, 1265, 1174, 907, 678. 


\section{General procedure for racemic alkylidene transfer}

Racemic alkylidenecyclopropanes were synhtesized according to the procedure summarized in Scheme 4 and described below.

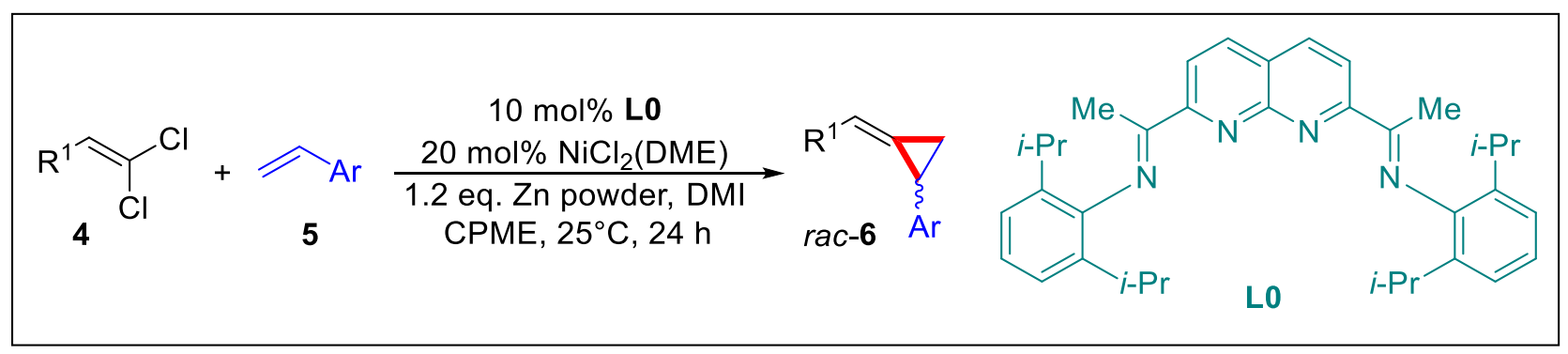

Scheme 4: Synhtetic procedure to access racemic alkylidenecyclopropanes.

According to a modified literature procedure. ${ }^{9}$ In a $\mathrm{N}_{2}$-filled glovebox, a 1/2 Dram glass vial equipped with a magnetic stir bar ( $3 \times 10 \mathrm{~mm})$ was charged with $\mathbf{L O}^{10}$ (10.0 mol\%, $\left.10.0 \mu \mathrm{mol}\right), \mathrm{NiCl}_{2}$ (DME) (20.0 mol\%, $20.0 \mu \mathrm{mol}), \mathrm{Zn}$ powder (1.20 equiv., $0.12 \mathrm{mmol})$, 1,3-dimethyl-2-imidazolidinone DMI (98.0 $\mu \mathrm{l}$, $0.90 \mathrm{mmol})$ and CPME $(160 \mu \mathrm{L})$. The reaction mixture was stirred $(1400 \mathrm{rpm})$ at room temperature for 10 minutes. A mixture of 1,1-dichloroalkene 4 (1.00 equiv., $0.10 \mathrm{mmol}$ ), alkene 5 (1.00 equiv., 0.10 mmol) and CPME (340 $\mu \mathrm{L})$ was added to the preformed catalyst. The vial was sealed and the reaction mixture was stirred (1400 rpm) at room temperature inside the glovebox for 24 hours. After this time, the reaction mixture was filtered over a short plug of $\mathrm{SiO}_{2}$ (eluting with $\mathrm{DCM}$ ) and concentrated under reduced pressure. The crude residue was purified by silica gel column chromatography (n-pentane:DCM) affording the products 6 as an inseparable mixture of $Z / E$ diastereomers.

Note1: Liquid reagents/reactants were degassed by freeze-pump-thaw $(3 \times)$ before being stored in the glovebox at $-30{ }^{\circ} \mathrm{C}$. Solid reagents/reactants were dried under high vacuum for $16 \mathrm{~h}$ before being stored in the glovebox at $-30{ }^{\circ} \mathrm{C}$.

Note2: The synthesized methylenecyclopropanes are prone to decomposition when kept under air at room temperature; therefore, after purification they were stored under a $\mathrm{N}_{2}$ atmosphere in a $-80{ }^{\circ} \mathrm{C}$ freezer. 


\section{Optimization of the enantioselective alkylidene transfer ${ }^{\mathrm{a}}$}
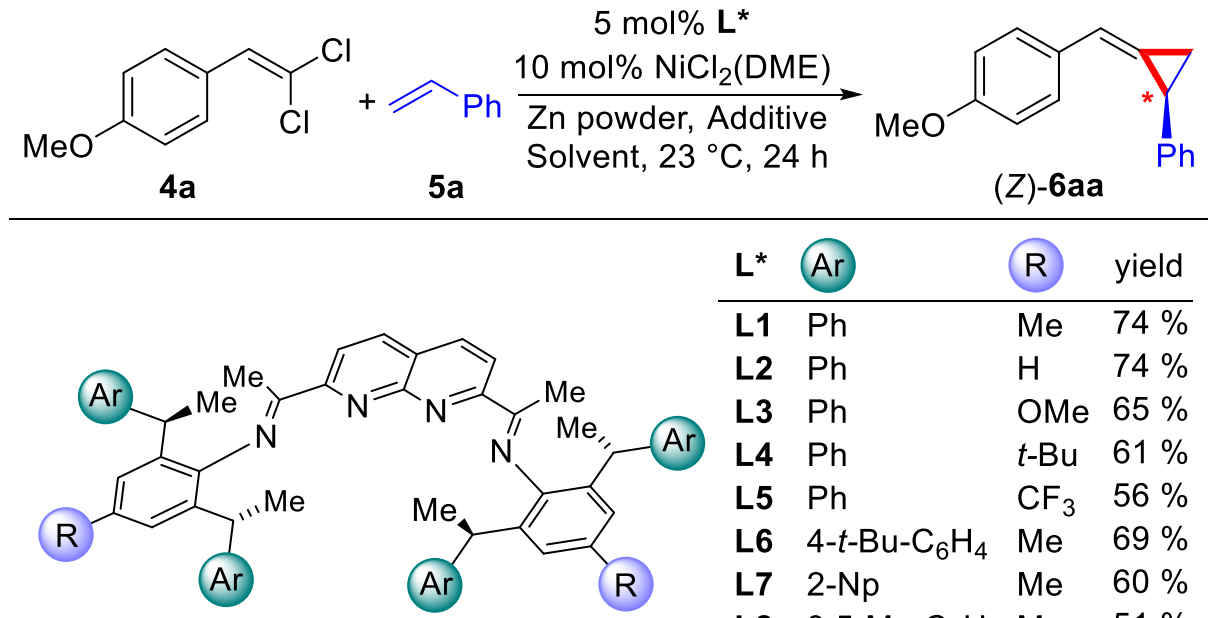

\begin{tabular}{llll} 
L* & $\mathrm{Ar}$ & $\mathrm{R}$ & yield \\
\hline L1 & $\mathrm{Ph}$ & $\mathrm{Me}$ & $74 \%$ \\
L2 & $\mathrm{Ph}$ & $\mathrm{H}$ & $74 \%$ \\
L3 & $\mathrm{Ph}$ & $\mathrm{OMe}$ & $65 \%$ \\
L4 & $\mathrm{Ph}$ & $t$-Bu & $61 \%$ \\
L5 & $\mathrm{Ph}$ & $\mathrm{CF}$ & $56 \%$ \\
L6 & $4-t-\mathrm{Bu}-\mathrm{C}_{6} \mathrm{H}_{4}$ & $\mathrm{Me}$ & $69 \%$ \\
$\mathbf{L} 7$ & $2-\mathrm{Np}$ & $\mathrm{Me}$ & $60 \%$ \\
L8 & $3,5-\mathrm{Me}-\mathrm{C}_{6} \mathrm{H}_{3} \mathrm{Me}$ & $51 \%$ \\
L9 & $3,5-\mathrm{F}-\mathrm{C}_{6} \mathrm{H}_{3}$ & $\mathrm{Me}$ & $67 \%$
\end{tabular}

\begin{tabular}{|c|c|c|c|c|c|c|c|}
\hline Entry & $\mathbf{L}^{*}$ & Solvent (M) & Additive & $\mathrm{T}\left({ }^{\circ} \mathrm{C}\right)$ & $\%$ Yield & $Z I E$ & $\operatorname{er}(Z)$ \\
\hline $1^{[\mathrm{b}]}$ & L1 & $\mathrm{Et}_{2} \mathrm{O}(0.13 \mathrm{M})$ & DMA & 25 & 61 & $2: 1$ & $84: 16$ \\
\hline 2 & L2 & $\mathrm{Et}_{2} \mathrm{O}(0.13 \mathrm{M})$ & DMA & 25 & 62 & $2.5: 1$ & $85: 15$ \\
\hline 3 & L3 & $\mathrm{Et}_{2} \mathrm{O}(0.13 \mathrm{M})$ & DMA & 25 & 50 & $2.5: 1$ & $85: 15$ \\
\hline 4 & L4 & $\mathrm{Et}_{2} \mathrm{O}(0.13 \mathrm{M})$ & DMA & 25 & 47 & $2.9: 1$ & $80: 20$ \\
\hline 5 & L5 & $\mathrm{Et}_{2} \mathrm{O}(0.13 \mathrm{M})$ & DMA & 25 & 26 & $2.4: 1$ & $81: 19$ \\
\hline 6 & L6 & $\mathrm{Et}_{2} \mathrm{O}(0.13 \mathrm{M})$ & DMA & 25 & 44 & $1.4: 1$ & $76: 24$ \\
\hline 7 & L7 & $\mathrm{Et}_{2} \mathrm{O}(0.13 \mathrm{M})$ & DMA & 25 & 31 & $1.4: 1$ & $66: 34$ \\
\hline 8 & L8 & $\mathrm{Et}_{2} \mathrm{O}(0.13 \mathrm{M})$ & DMA & 25 & 18 & $1.8: 1$ & $74: 26$ \\
\hline 9 & L9 & $\mathrm{Et}_{2} \mathrm{O}(0.13 \mathrm{M})$ & DMA & 25 & 23 & $3.9: 1$ & $87: 13$ \\
\hline $10^{[\mathrm{c}]}$ & L9 & $\mathrm{Et}_{2} \mathrm{O}(0.13 \mathrm{M})$ & DMA & 25 & 71 & $3.9: 1$ & $96: 4$ \\
\hline $11^{[c]}$ & L9 & $\mathrm{Et}_{2} \mathrm{O}(0.20 \mathrm{M})$ & DMA & 25 & 46 & $3.3: 1$ & $95: 5$ \\
\hline $12^{[\mathrm{c}]}$ & L9 & CPME $(0.20 \mathrm{M})$ & DMA & 25 & 53 & $3.1: 1$ & $94: 6$ \\
\hline $13^{[\mathrm{c}][\mathrm{d}]}$ & L9 & CPME (0.20 M) & HMPA & 25 & 72 & $4.2: 1$ & $93: 7$ \\
\hline $14^{[c][d]}$ & L9 & CPME $(0.20 \mathrm{M})$ & HMPA & 10 & 74 & $4.7: 1$ & $94: 6$ \\
\hline $15^{[\mathrm{c}][\mathrm{d}]}$ & L9 & CPME $(0.20 \mathrm{M})$ & HMPA & 0 & 69 & $3.4: 1$ & $96: 4$ \\
\hline $16^{[\mathrm{c}[\mathrm{d}]}$ & L9 & CPME (0.20 M) & HMPA & -15 & 39 & $2.8: 1$ & $95: 5$ \\
\hline $17^{[\mathrm{c}][\mathrm{d}][\mathrm{e}]}$ & L9 & CPME $(0.20 \mathrm{M})$ & HMPA & 0 & 49 & 3.3:1 & $94: 6$ \\
\hline $18^{[\mathrm{c}][\mathrm{d}][\mathrm{f}]}$ & L9 & CPME (0.20 M) & HMPA & 0 & 30 & $4.6: 1$ & $92: 8$ \\
\hline $19^{[\mathrm{c}][\mathrm{g}]}$ & L9 & CPME (0.20 M) & DMPU & 0 & traces & - & - \\
\hline $20^{[c][h]}$ & L9 & CPME (0.20 M) & DMI & 0 & 88 & $3: 1$ & $94: 6$ \\
\hline $21^{[\mathrm{h}][i]}$ & L9 & CPME (0.20 M) & DMI & 0 & 89 & $3.8: 1$ & $96: 4$ \\
\hline
\end{tabular}

[a] Conditions: $20.3 \mathrm{mg}(0.10 \mathrm{mmol})$ 4a, $10.4 \mathrm{mg}(0.10 \mathrm{mmol}) \mathbf{5 a}, 2.2 \mathrm{mg}(10.0 \mu \mathrm{mol}) \mathrm{NiCl}_{2}(\mathrm{DME}), 5.00 \mu \mathrm{mol} \mathrm{L}^{*}, 19.6 \mathrm{mg}(0.30 \mathrm{mmol}) \mathrm{Zn}$ powder, $93.0 \mu \mathrm{l}(1.00$ $\mathrm{mmol}) \mathrm{DMA}$ in $\mathrm{Et}_{2} \mathrm{O}$ at $23^{\circ} \mathrm{C}$ for $24 \mathrm{~h}$; [b] when $Z-6 \mathrm{aa}(84: 16 \mathrm{er})$ was resubmitted to the reaction conditions, no isomerization to $E-6$ aa and no change in enantiomeric ratio were observed; [c] with $7.2 \mathrm{mg}(0.11 \mathrm{mmol}) \mathrm{Zn}$ powder; [d] with $157 \mu \mathrm{l}(0.9 \mathrm{mmol}) \mathrm{HMPA}$; [e] with $30.5 \mathrm{mg}(0.15 \mathrm{mmol}) 4 \mathrm{a}$; [f] with $15.6 \mathrm{mg}(0.15 \mathrm{mmol}) 5 \mathrm{a}$; [g] with $109 \mu \mathrm{l}(0.9 \mathrm{mmol}) \mathrm{DMPU}$; [h] with $98.0 \mu \mathrm{l}(0.9 \mathrm{mmol}) \mathrm{DMl}$; [i] with $3.5 \mathrm{mg}(16.0 \mu \mathrm{mol}) \mathrm{NiCl}_{2}(\mathrm{DME}), 7.6 \mathrm{mg}(8.00 \mu \mathrm{mol}) \mathrm{L9}, 7.6 \mathrm{mg}(116 \mu \mathrm{mol}) \mathrm{Zn} \mathrm{powder}$. 


\section{General procedure for enantioselective alkylidene transfer (GP3)}

Chiral alkylidenecyclopropanes 6 were synhtesized according to the procedure summarized in Scheme 6 and described below.

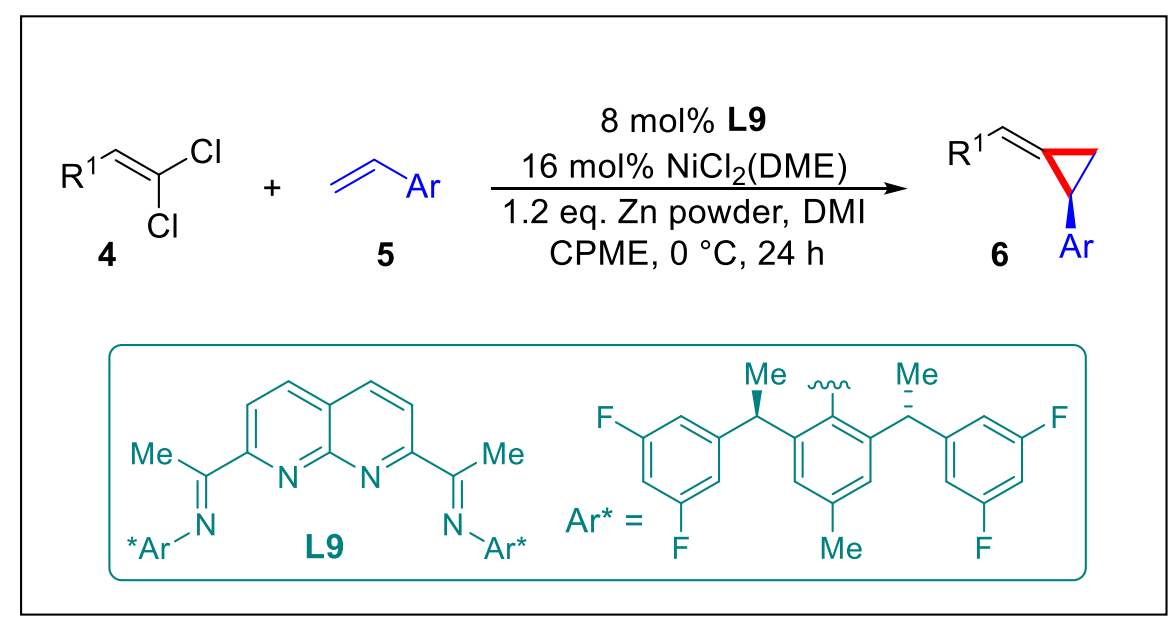

Scheme 6: Synhtetic procedure to access chiral alkylidenecyclopropanes 6.

In a $\mathrm{N}_{2}$-filled glovebox, a 1/2 Dram glass vial equipped with a magnetic stir bar (3 x $\left.10 \mathrm{~mm}\right)$ was charged with $\mathbf{L 9}(8.00 \mathrm{~mol} \%, 8.00 \mu \mathrm{mol}), \mathrm{NiCl}_{2}$ (DME) $(16.0 \mathrm{~mol} \%, 16.0 \mu \mathrm{mol}), \mathrm{Zn}$ powder (1.16 equiv., 116 $\mu \mathrm{mol})$, 1,3-dimethyl-2-imidazolidinone DMI (98.0 $\mu \mathrm{l}, 0.90 \mathrm{mmol})$ and CPME (160 $\mu \mathrm{L})$. The reaction mixture was stirred $(1400 \mathrm{rpm})$ at room temperature for 15 minutes. A mixture of 1,1-dichloroalkene 4 (1.00 equiv., $0.10 \mathrm{mmol}$ ), alkene 5 (1.00 equiv., $0.10 \mathrm{mmol})$ and CPME (340 $\mu \mathrm{L})$ was added to the preformed catalyst. The vial was sealed, transferred out of the glovebox into a flame-dried Schlenk flask (with sand as heat transfer medium) and the reaction mixture was stirred $(1400 \mathrm{rpm})$ at $0{ }^{\circ} \mathrm{C}$ for 24 hours (see Figure 1 for description of the reaction apparatus). After this time, the reaction mixture was filtered over a short plug of $\mathrm{SiO}_{2}$ (eluting with DCM) and concentrated under reduced pressure. The crude residue was purified by silica gel column chromatography ( $n$-pentane:DCM) affording the products 6 as an inseparable mixture of $Z / E$ diastereomers.

Note1: Liquid reagents/reactants were degassed by freeze-pump-thaw $(3 \times)$ before being stored in the glovebox at $-30{ }^{\circ} \mathrm{C}$. Solid reagents/reactants were dried under high vacuum for $16 \mathrm{~h}$ before being stored in the glovebox at $-30{ }^{\circ} \mathrm{C}$.

Note2: The synthesized methylenecyclopropanes are prone to decomposition when kept under air at room temperature; therefore, after purification they were stored under a $\mathrm{N}_{2}$ atmosphere in a $-80{ }^{\circ} \mathrm{C}$ freezer.

Note3: The Z/E stereochemistry of alkylidenecyclopropanes 6 was assigned on the basis of a previous literature report. ${ }^{9}$ 

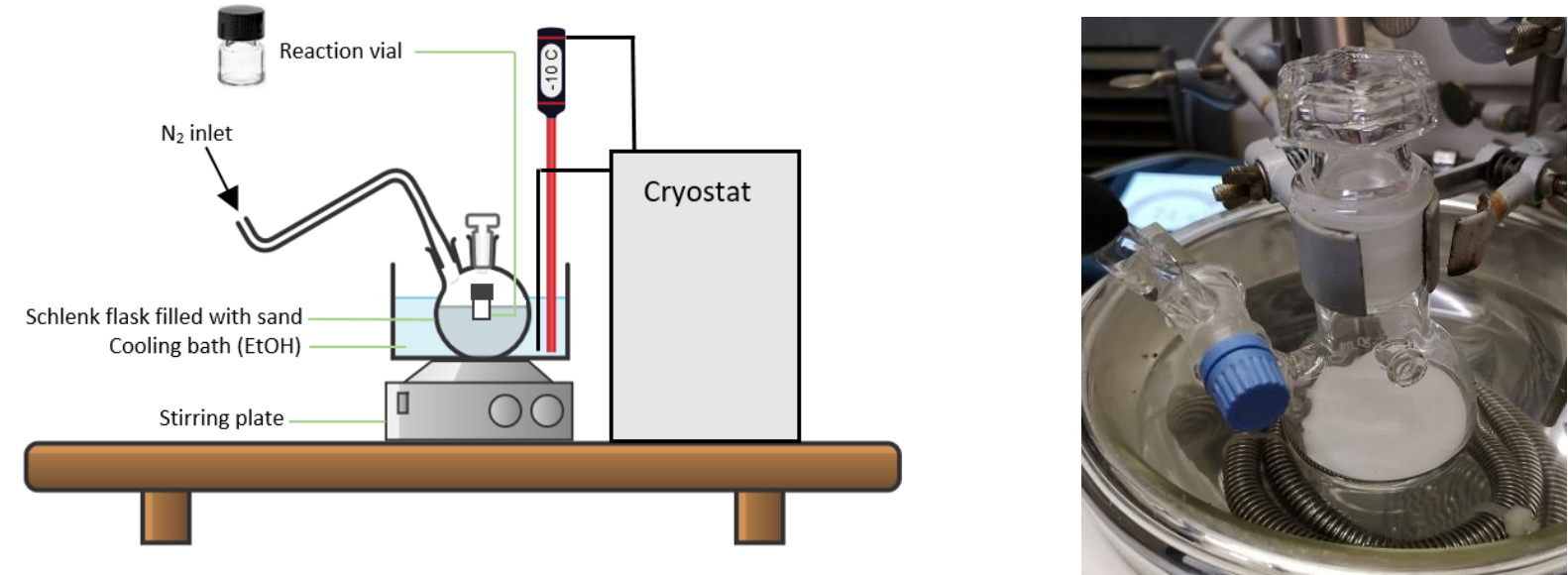

Figure 1: Description of the reaction apparatus used for asymmetric alkylidenecyclopropanation of olefins. 


\section{Characterization data of chiral Alkylidenecyclopropanes 6}

\section{(R)-1-Methoxy-4-((2-phenylcyclopropylidene)methyl)benzene 6aa}

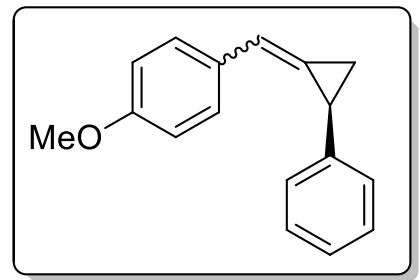

Prepared according to $\underline{\mathbf{G P 3}}$.

Yield: $89 \%$

$\mathbf{R}_{\boldsymbol{f}}(20 \%$ DCM in pentane): 0.25

Appearance: colourless oil

$Z / E$ ratio: $3.8: 1$

${ }^{1}$ H NMR (400 MHz, Chloroform-d): $\delta 7.52$ (d, 2H, E-6aa), $7.34-7.06$ (m, 5H E-6aa + 7H Z-6aa), $6.90(\mathrm{~d}, J=8.7 \mathrm{~Hz}, 2 \mathrm{H}, \boldsymbol{E}$-6aa), 6.87 (q, $J=2.1 \mathrm{~Hz}, 1 \mathrm{H}, \boldsymbol{Z}$-6aa), 6.84 (q, $J=2.3 \mathrm{~Hz}, 1 \mathrm{H}, \boldsymbol{E}$-6aa), 6.76 (d, $J=8.7 \mathrm{~Hz}, 2 \mathrm{H} \mathrm{Z}$-6aa), 3.82 (s, 3H, $\boldsymbol{E}$-6aa), 3.74 (s, 3H, Z-6aa), 2.86 (ddd, $J$ = 9.0, 4.6, $2.1 \mathrm{~Hz}, 1 \mathrm{H}, \boldsymbol{Z}$ 6aa), $2.67(\mathrm{ddd}, J=8.8,4.7,1.7 \mathrm{~Hz}, 1 \mathrm{H}, \boldsymbol{E}$-6aa), $2.05(\mathrm{td}, J=9.0,2.4 \mathrm{~Hz}, 1 \mathrm{H}, \boldsymbol{E}$-6aa), $1.81(\mathrm{td}, J=8.9$, $2.0 \mathrm{~Hz}, 1 \mathrm{H}, \boldsymbol{Z}$-6aa), 1.49 (ddd, $J=9.2,4.7,2.4 \mathrm{~Hz}, 1 \mathrm{H}, \boldsymbol{E}$-6aa), $1.25-1.18$ (ddd, 1H, Z-6aa).

${ }^{13}$ C NMR (101 MHz, Chloroform-d): $\delta 159.00$ (Z-6aa), 158.83 (E-6aa), 142.49 (Z-6aa), 141.50 (E6aa), 130.70 (Z-6aa), 130.34 (E-6aa), 128.69 (E-6aa), 128.49 (Z-6aa), 128.29 (E-6aa), 128.13 (Z-6aa), 126.57 (Z-6aa), 126.52 (E-6aa), 126.26 (Z-6aa), 126.03 (Z-6aa), 126.00 (E-6aa), 125.02 (E-6aa), 119.58 (E-6aa), 118.88 (Z-6aa), 114.13 (E-6aa), 114.06 (Z-6aa), 55.46 (Z-6aa), 55.34 (E-6aa), 21.48 (E-6aa), 18.07 (Z-6aa), 16.02 (Z-6aa), 13.98 (E-6aa).

IR (ATR): $v\left(\mathrm{~cm}^{-1}\right)$ 3061, 3032, 2919, 2838, 1781, 1740, 1603, 1513, 1251, 1169, 1031, 700.

HRMS (APPI/LQT-Orbitrap): calcd for $\mathrm{C}_{17} \mathrm{H}_{15} \mathrm{O}^{+}\left[\mathrm{M}+\mathrm{H}_{-1}\right]^{+}: 235.1117$; found: 235.1114 .

$[\boldsymbol{\alpha}] \mathbf{D}^{\mathbf{2 0}}=-409\left(c=1.0, \mathrm{CHCl}_{3}\right)$.

e.r. $(\boldsymbol{Z})$ : 96:4 (Reverse Phase HPLC, Chiralpak IB, $\mathrm{H}_{2} \mathrm{O} / \mathrm{ACN}=62 / 38, \lambda=260 \mathrm{~nm}, 0.75 \mathrm{~mL} / \mathrm{min}, \mathrm{t}_{\mathrm{R}}$ major $=90.1 \mathrm{~min}, \mathrm{t}_{\mathrm{R}}$ minor $=92.2 \mathrm{~min}$ ) 

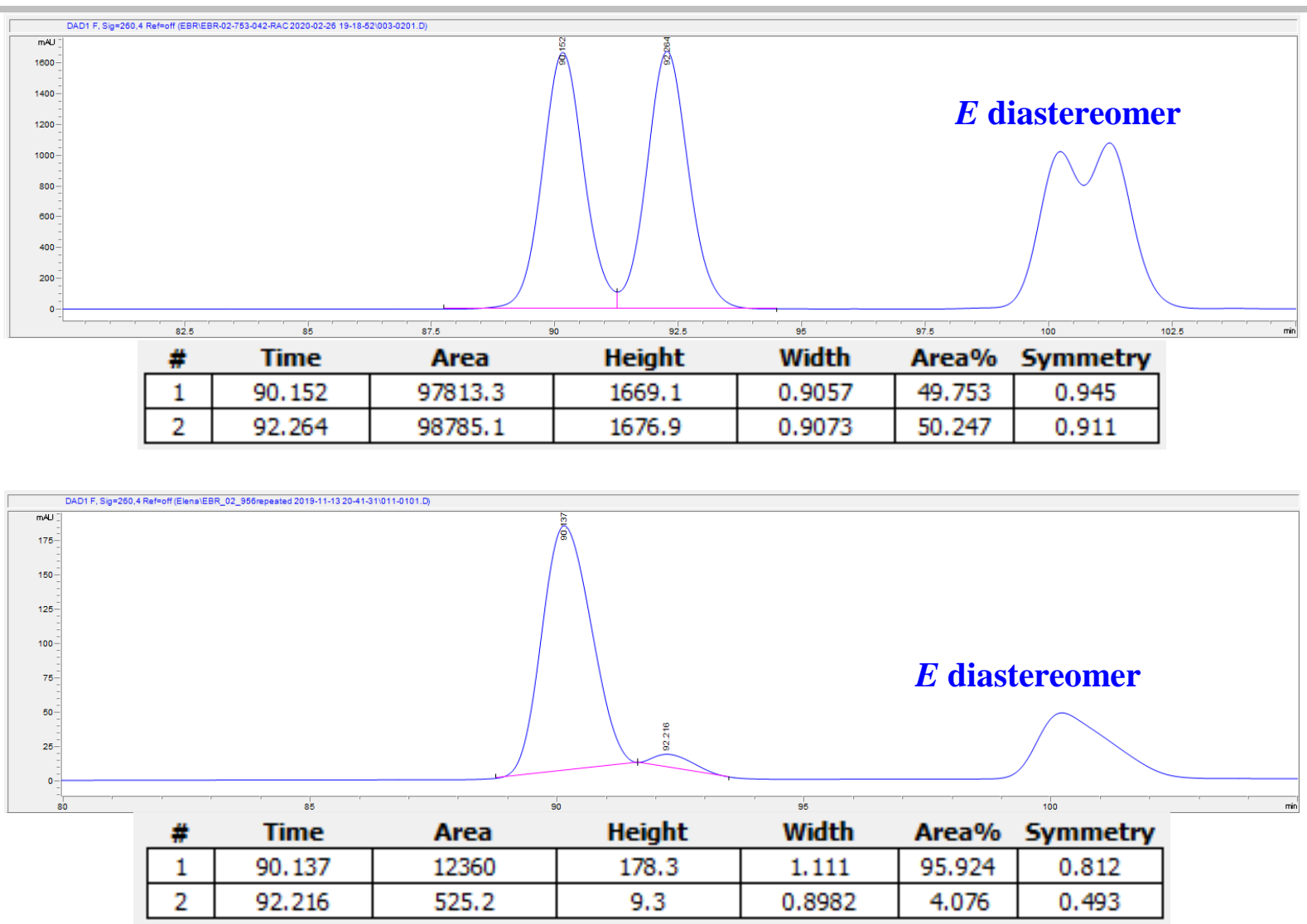


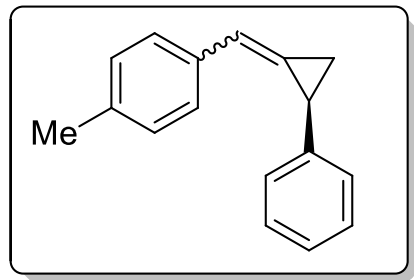

Prepared according to $\underline{\text { GP3 }}$.

Yield: $97 \%$

$\mathbf{R}_{f}$ (20\% DCM in pentane): 0.50

Appearance: yellow oil

Z/E ratio: 3.0:1

${ }^{1} \mathbf{H}$ NMR (400 MHz, Chloroform- $d$ ): $\delta 7.50(\mathrm{~d}, J=7.9 \mathrm{~Hz}, 2 \mathrm{H}, \boldsymbol{E}$-6ba), $7.32-7.10(\mathrm{~m}, 7 \mathrm{H} \boldsymbol{E}-6 \mathbf{b a}+7 \mathrm{H}$ Z-6ba), 7.05 (d, $J=7.8 \mathrm{~Hz}, 2 \mathrm{H}, \boldsymbol{Z}$-6ba), 6.91 (q, $J=2.1 \mathrm{~Hz}, 1 \mathrm{H}, \boldsymbol{Z}$-6ba), 6.89 (q, $J=2.3 \mathrm{~Hz}, 1 \mathrm{H}, \boldsymbol{E}$-6ba), 2.90 (ddd, $J=9.1,4.8,2.0 \mathrm{~Hz}, 1 \mathrm{H}, \boldsymbol{Z}$-6ba), 2.70 (ddd, $J=9.1,4.8,1.6 \mathrm{~Hz}, 1 \mathrm{H}, \boldsymbol{E}$-6ba), 2.38 (s, 3H, $\boldsymbol{E}$ 6ba), 2.29 (s, 3H, Z-6ba), 2.09 (td, $J=9.1,2.4 \mathrm{~Hz}, 1 \mathrm{H}, \boldsymbol{E}$-6ba), 1.84 (td, $J=9.0,2.0 \mathrm{~Hz}, 1 \mathrm{H}, \boldsymbol{Z}-\mathbf{6 b a}$ ), $1.53-1.50$ (m, $1 \mathrm{H}, \boldsymbol{E}$-6ba), $1.27-1.21$ (m, $1 \mathrm{H}, \boldsymbol{Z}$-6ba).

${ }^{13}$ C NMR (101 MHz, Chloroform-d): $\delta 142.41$ (E-6ba), 141.41 (Z-6ba), 137.04 (E-6ba), 136.83 (Z6ba), 135.04 (E-6ba), 134.64 (Z-6ba), 129.38, 129.32, 128.68, 128.50, 127.60, 127.07, 126.92, 126.58, 126.55, 126.40, 126.05, 126.03, 120.07 (Z-6ba), 119.37 (E-6ba), 21.58 (Z-6ba), 21.39 (E-6ba), 21.30 (Z-6ba), 18.06 (E-6ba), 16.12 (E-6ba), 13.88 (Z-6ba).

IR (ATR): $v\left(\mathrm{~cm}^{-1}\right)$ 3027, 3002, 2965, 2921, 1603, 1511, 1493, 1452, 853, 809, 748, 697, 504.

HRMS (APPI/QTOF): calcd for $\mathrm{C}_{17} \mathrm{H}_{16}{ }^{+}[\mathrm{M}]^{+}: 220.1247$; found: 220.1240 .

$[\boldsymbol{\alpha}]_{\mathbf{D}^{20}}=-358\left(c=1.0, \mathrm{CHCl}_{3}\right)$.

e.r. (Z): 92:8 (Reverse Phase HPLC, Chiralpak IB, $\mathrm{H}_{2} \mathrm{O} / \mathrm{ACN}=$ gradient from $60 / 40$ to $40 / 60, \lambda=260$ $\mathrm{nm}, 1.00 \mathrm{~mL} / \mathrm{min}, \mathrm{t}_{\mathrm{R}}$ major $=50.4 \mathrm{~min}, \mathrm{t}_{\mathrm{R}} \operatorname{minor}=52.9 \mathrm{~min}$ ) 

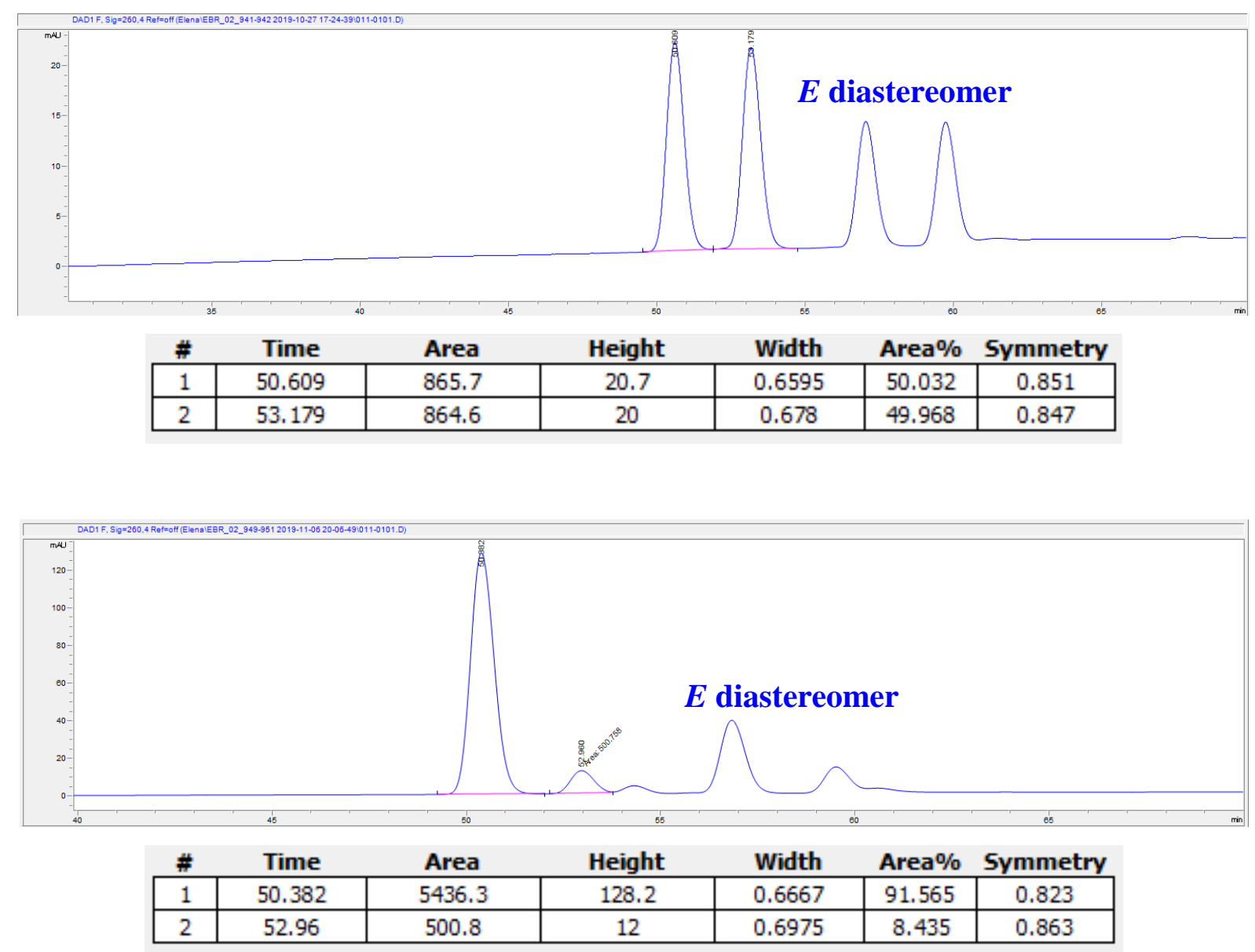


\section{$\underline{\text { (R)-Triisopropyl(4-((2-phenylcyclopropylidene)methyl)phenoxy)silane 6ca }}$}

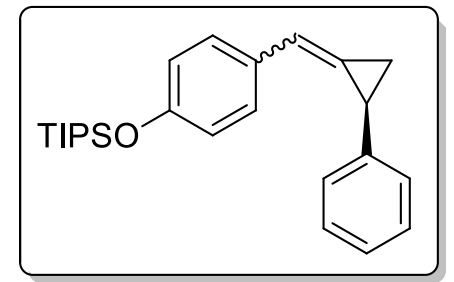

Prepared according to $\underline{\mathbf{G P 3}}$.

Yield: $93 \%$

$\mathbf{R}_{\boldsymbol{f}}(2 \%$ EtOAc in pentane): 0.34

Appearance: colourless oil

Z/E ratio: $2.6: 1$

${ }^{1}$ H NMR (400 MHz, Chloroform-d): $\delta 7.46(\mathrm{~d}, J=8.5 \mathrm{~Hz}, 2 \mathrm{H}, \boldsymbol{E}-\mathbf{6 c a}), 7.30-7.17(\mathrm{~m}, 5 \mathrm{H} \boldsymbol{E}-\mathbf{6 c a}+5 \mathrm{H}$ Z-6ca), 7.13 (d, $J=6.8 \mathrm{~Hz}, 2 \mathrm{H}, Z$-6ca), $6.92-6.86$ (m, 2H E-6ca + 1H Z-6ca), 6.85 (q, $J=2.2 \mathrm{~Hz}, 1 \mathrm{H}$, $\boldsymbol{E}$-6ca), 6.75 (d, $J=8.6 \mathrm{~Hz}, 2 \mathrm{H}, \boldsymbol{Z}$-6ca), 2.87 (ddd, $J=9.0,4.6,2.0 \mathrm{~Hz}, 1 \mathrm{H}, \boldsymbol{Z}$-6ca), 2.68 (ddd, $J=9.0$, 4.7, $1.7 \mathrm{~Hz}, 1 \mathrm{H}, \boldsymbol{E}$-6ca), 2.06 (td, $J=9.0,2.4 \mathrm{~Hz}, 1 \mathrm{H}, \boldsymbol{E}$-6ca), 1.81 (td, $J=8.9,2.0 \mathrm{~Hz}, 1 \mathrm{H}, Z$-6ca), 1.50 (ddd, $J=9.2,4.7,2.4 \mathrm{~Hz}, 1 \mathrm{H}, \boldsymbol{E}$-6ca), $1.35-1.16$ (m, 3H E-6ca + 4H Z-6ca), 1.13 (d, J = 7.4 Hz, 18H, E-6ca), 1.07 (d, $J=7.8 \mathrm{~Hz}, 18 \mathrm{H}, \boldsymbol{Z}$-6ca).

${ }^{13}$ C NMR (101 MHz, Chloroform-d): $\delta 155.55$ (E-6ca), 155.39 (Z-6ca), 142.53 (E-6ca), 141.58 (Z6ca), 130.89 (E-6ca), 130.57 (Z-6ca), 128.67, 128.48, 128.23, 128.06, 126.60, 126.59, 126.09, 126.01, 125.98, 124.90, 120.12 (E-6ca), 120.05 (Z-6ca), 119.82 (Z-6ca), 119.04 (E-6ca), 21.49 (Z-6ca), 18.09 (E-6ca), 18.04 (Z-6ca), 16.06 (E-6ca), 14.00 (Z-6ca), 12.84 (E-6ca), 12.78 (Z-6ca).

IR (ATR): $v\left(\mathrm{~cm}^{-1}\right)$ 2943, 2891, 2865, 1602, 1507, 1261, 907, 882, 695, 680.

HRMS (ESI/QTOF): calcd for $\mathrm{C}_{25} \mathrm{H}_{35} \mathrm{OSi}^{+}[\mathrm{M}+\mathrm{H}]^{+}: 379.2452$; found: 379.2453 .

$[\boldsymbol{\alpha}]_{\mathbf{D}^{\mathbf{2 0}}}=-226\left(c=1.0, \mathrm{CHCl}_{3}\right)$.

e.r. $(\boldsymbol{Z})$ : $87: 13$ (Chiralpak IG, Hexane/IPA $=90 / 10, \lambda=254 \mathrm{~nm}, 1.00 \mathrm{~mL} / \mathrm{min}, \mathrm{t}_{\mathrm{R}}$ major $=5.93 \mathrm{~min}, \mathrm{t}_{\mathrm{R}}$ minor $=6.90 \mathrm{~min}$, determined after derivatization to the corresponding alcohol 13) 


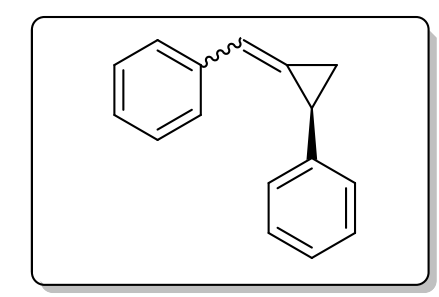

Prepared according to $\underline{\text { GP3 }}$.

Yield: 63\%

$\mathbf{R}_{f}$ (20\% DCM in pentane): 0.50

Appearance: yellow oil

Z/E ratio: 1.7:1

${ }^{1}$ H NMR (400 MHz, Chloroform- $\boldsymbol{d}$ ): $\delta 7.60$ (d, $J=7.5 \mathrm{~Hz}, 2 \mathrm{H}, \boldsymbol{E}$-6da), 7.38 (m, 2H $\boldsymbol{E}$-6da + 2H $\boldsymbol{Z}$ 6da), $7.31-7.09$ (m, $6 \mathrm{H} \boldsymbol{E}$-6da + 8H Z-6da), $6.96-6.93$ (m, 1H, Z-6da), $6.93-6.90$ (m, 1H, $\boldsymbol{E}$-6da), 2.92 (ddd, $J=9.2,4.8,2.0 \mathrm{~Hz}, 1 \mathrm{H}, \boldsymbol{Z}$-6da), 2.72 (ddd, $J=9.2,4.9,1.7 \mathrm{~Hz}, 1 \mathrm{H}, \boldsymbol{E}$-6da), 2.11 (td, $J=9.2$, $2.4 \mathrm{~Hz}, 1 \mathrm{H} \boldsymbol{E}$-6da), 1.85 (td, $J=9.1,2.0 \mathrm{~Hz}, 1 \mathrm{H}, \boldsymbol{Z}$-6da), $1.60-1.53$ (m, $1 \mathrm{H}, \boldsymbol{E}$-6da), $1.31-1.23$ (m, $1 \mathrm{H}$, Z-6da).

${ }^{13}$ C NMR (101 MHz, Chloroform-d): $\delta 142.22$ (E-6da), 141.26 (Z-6da), 137.80 (E-6da), 137.36 (Z6da), 128.77, 128.71, 128.68, 128.61, 128.53, 127.65, 127.27, 127.15, 127.08, 127.02, 126.59, 126.54, 126.12, 126.09, 120.27 (Z-6da), 119.53 (E-6da), 21.64 (Z-6da), 18.05 (E-6da), 16.11 (E-6da), 13.88 (Z6da).

IR (ATR): $v\left(\mathrm{~cm}^{-1}\right)$ 3082, 3060, 3027, 2966, 2924, 1493, 1450, 750, 693.

HRMS (APPI/QTOF): calcd for $\mathrm{C}_{16} \mathrm{H}_{14}{ }^{+}[\mathrm{M}]^{+}:$206.1090; found: 206.1081 .

$[\boldsymbol{\alpha}]_{\mathbf{D}^{20}}=-289\left(c=1.0, \mathrm{CHCl}_{3}\right)$.

e.r. (Z): 88:12 (Reverse Phase HPLC, Chiralpak IB, $\mathrm{H}_{2} \mathrm{O} / \mathrm{ACN}=$ gradient from 60/40 to 40/60, $\lambda=260$ $\mathrm{nm}, 1.00 \mathrm{~mL} / \mathrm{min}, \mathrm{t}_{\mathrm{R}}$ major $=43.7 \mathrm{~min}, \mathrm{t}_{\mathrm{R}}$ minor $=46.3 \mathrm{~min}$ ) 

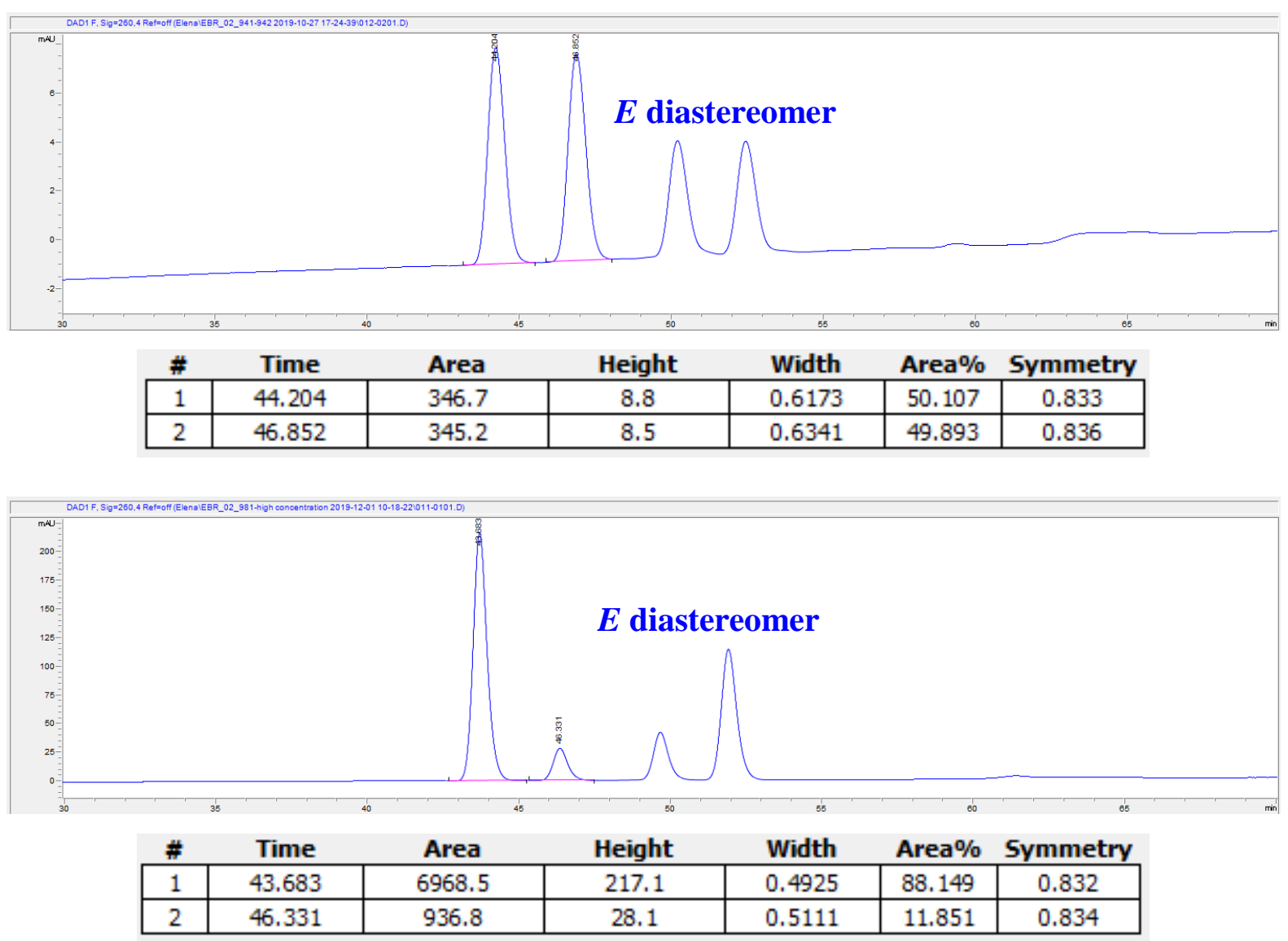


\section{$\underline{\text { (R)-1-((2-Phenylcyclopropylidene)methyl)-4-(trifluoromethyl)benzene Gea }}$}

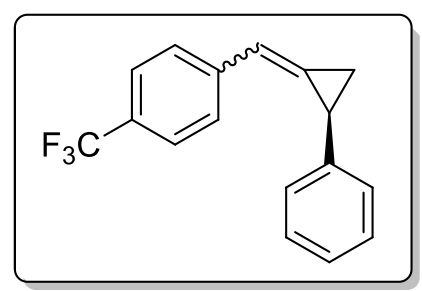

Prepared according to $\underline{\mathbf{G P 3}}$.

Yield: $86 \%$

$\mathbf{R}_{f}(100 \%$ pentane): 0.29

Appearance: light yellow oil

Z/E ratio: $3.0: 1$

${ }^{1}$ H NMR (400 MHz, Chloroform-d): $\delta 7.68(\mathrm{~d}, J=8.2 \mathrm{~Hz}, 2 \mathrm{H}, \boldsymbol{E}$-6ea), 7.60 (d, $J=8.2 \mathrm{~Hz}, 2 \mathrm{H}, \boldsymbol{E}$-6ea), 7.51 - 7.41 (m, 4H, Z-6ea), 7.31 - 7.15 (m, 5H E-6еa + 3H Z-6ea), 7.13 - 7.07 (m, 2H, Z-6ea), 6.97 (q, $J=2.1 \mathrm{~Hz}, 1 \mathrm{H}, \boldsymbol{Z}$-6ea), 6.94 (q, 1H, E-6ea), $2.94(\mathrm{ddd}, J=9.5,5.1,2.1 \mathrm{~Hz}, 1 \mathrm{H}, \boldsymbol{Z}$-6ea), 2.76 (ddd, $J=$ 9.4, 5.2, $1.7 \mathrm{~Hz}, 1 \mathrm{H}, \boldsymbol{E}$-6ea), $2.13(\mathrm{td}, J=9.5,2.4 \mathrm{~Hz}, 1 \mathrm{H}, \boldsymbol{E}$-6ea), $1.90(\mathrm{td}, J=9.4,2.0 \mathrm{~Hz}, 1 \mathrm{H}, \boldsymbol{Z}$-6ea), 1.59 (ddd, $J=9.6,5.2,2.4 \mathrm{~Hz}, 1 \mathrm{H}, \boldsymbol{E}$-6ea), 1.33 (ddd, $J=9.5,5.1,2.0 \mathrm{~Hz}, 1 \mathrm{H}, Z$-6ea).

${ }^{13}$ C NMR (101 MHz, Chloroform- $d$ ): $\delta$ 141.56, 141.21, 140.71, 140.60, 132.05, 131.20, 129.11 (q, $J=$ $33.3 \mathrm{~Hz}, \mathbf{Z}-6 \mathbf{e a}), 128.86,128.62,127.18,127.06,126.61,126.41,126.33,125.71$ (q, $J=5.0 \mathrm{~Hz}, \boldsymbol{E}$-6ea), 125.55 (q, $J=4.0 \mathrm{~Hz}, Z$-6ea), 124.38 (q, $J=272.7 \mathrm{~Hz}, \boldsymbol{Z}$-6ea), 119.10, 118.50, 21.70 (Z-6ea), $18.20(\boldsymbol{E}$ 6еa), 15.91 (E-6ea), 13.93 (Z-6ea).

${ }^{19}$ F NMR (376 MHz, Chloroform-d): $\delta$-62.41 (E-6ea), -62.46 (Z-6ea).

IR (ATR): $v\left(\mathrm{~cm}^{-1}\right)$ 3030, 2926, 2855, 1616, 1323, 1164, 1122, 1068, 860, 697.

HRMS (ESI/LQT-Orbitrap): calcd for $\mathrm{C}_{17} \mathrm{H}_{13} \mathrm{~F}_{3}{ }^{+}[\mathrm{M}]^{+}:$274.0964; found: 274.0958 .

$[\boldsymbol{\alpha}]_{\mathbf{D}^{\mathbf{2 0}}}=-263\left(c=1.0, \mathrm{CHCl}_{3}\right)$.

e.r. $(\boldsymbol{Z})$ : 94:6 (Reverse Phase HPLC, Chiralpak IB, $\mathrm{H}_{2} \mathrm{O} / \mathrm{ACN}=$ gradient from $60 / 40$ to $40 / 60, \lambda=280$ $\mathrm{nm}, 1.00 \mathrm{~mL} / \mathrm{min}, \mathrm{t}_{\mathrm{R}}$ major $=54.8 \mathrm{~min}, \mathrm{t}_{\mathrm{R}}$ minor $\left.=56.3 \mathrm{~min}\right)$ 

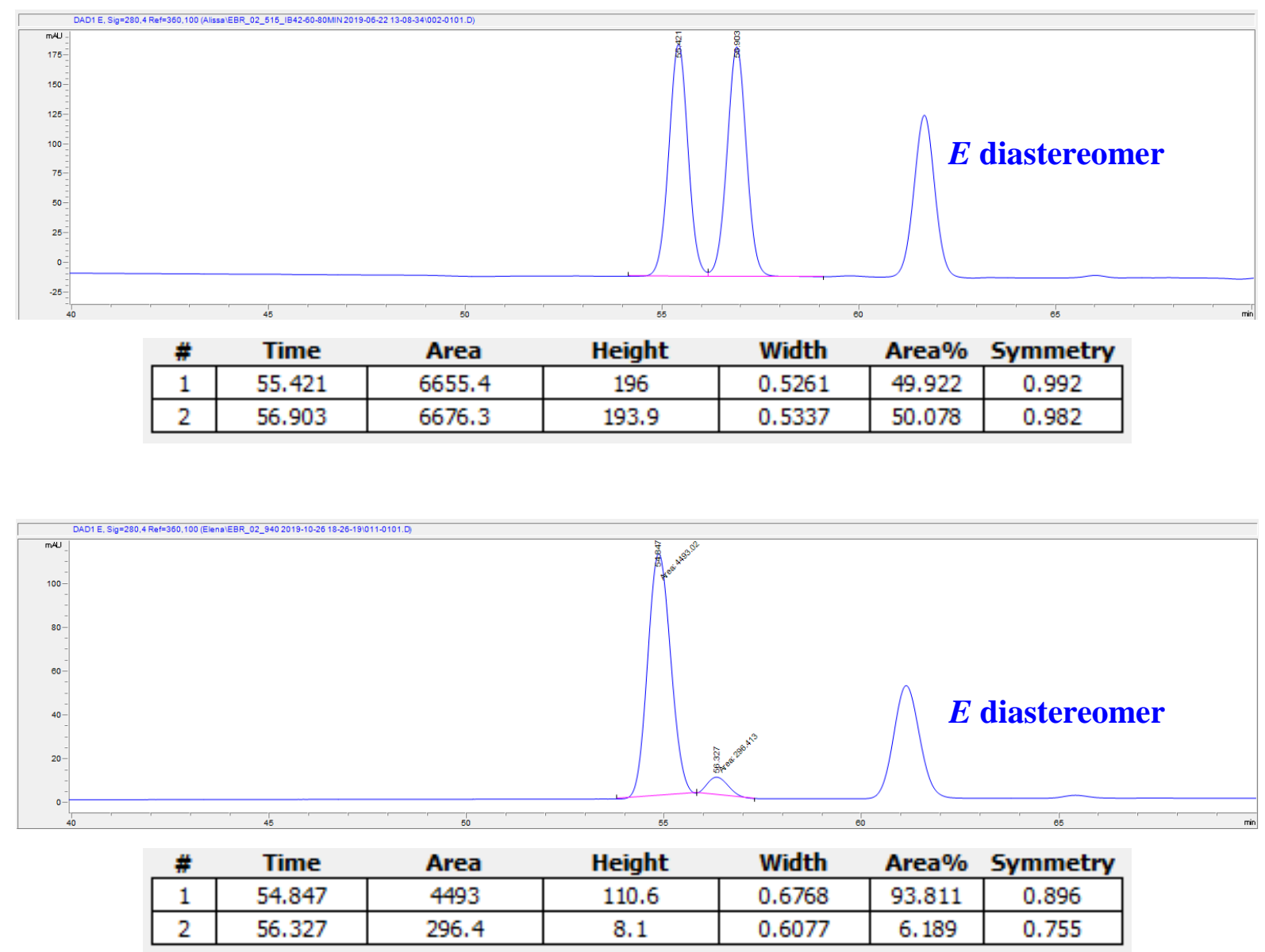


\section{(R)-Methyl 4-((2-phenylcyclopropylidene)methyl)benzoate 6fa}

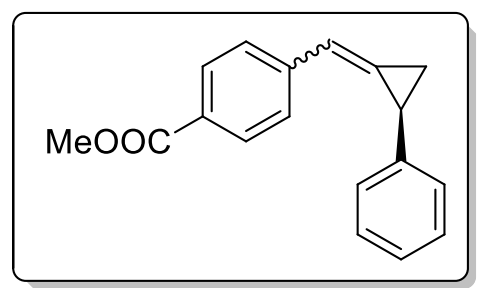

Prepared according to $\underline{\mathbf{G P 3}}$.

Yield: $96 \%$

$\mathbf{R}_{f}(20 \%$ DCM in pentane): 0.46

Appearance: light yellow solid

$Z / E$ ratio: $4.6: 1$

${ }^{1}$ H NMR (400 MHz, Chloroform-d): $\delta 8.03$ (d, $J=7.7 \mathrm{~Hz}, 2 \mathrm{H}, \boldsymbol{E}$-6fa), 7.90 (d, $J=7.7 \mathrm{~Hz}, 2 \mathrm{H}, \boldsymbol{Z}$-6fa), $7.64(\mathrm{~d}, J=8.1 \mathrm{~Hz}, 2 \mathrm{H}, \boldsymbol{E}$-6fa), $7.42(\mathrm{~d}, J=8.3 \mathrm{~Hz}, 2 \mathrm{H}, \boldsymbol{Z}$-6fa) $) 7.33-7.15$ (m, 5H $\boldsymbol{E}$-6fa $+3 \mathrm{H} \mathrm{Z}$-6fa), $7.10(\mathrm{~d}, J=7.0 \mathrm{~Hz}, 2 \mathrm{H}, \boldsymbol{Z}-\mathbf{6 f a}), 7.00-6.97$ (m, 1H, Z-6fa), $6.97-6.94$ (m, 1H, $\boldsymbol{E}$-6fa), 3.93 (s, 3H, $\boldsymbol{E}$ 6fa), 3.87 (s, 3H, Z-6fa), $2.95(\mathrm{ddd}, J=9.5,5.1,2.1 \mathrm{~Hz}, 1 \mathrm{H}, \boldsymbol{Z}-\mathbf{- 6 f a}), 2.75(\mathrm{ddd}, J=9.3,5.2,1.7 \mathrm{~Hz}, 1 \mathrm{H}$, $\boldsymbol{E}$-6fa), $2.14(\mathrm{td}, J=9.5,2.4 \mathrm{~Hz}, 1 \mathrm{H}, \boldsymbol{E}$-6fa), $1.89(\mathrm{td}, J=9.4,2.0 \mathrm{~Hz}, 1 \mathrm{H}, \boldsymbol{Z}$-6fa), 1.60 (ddd, $J=9.8,5.3$, $2.5 \mathrm{~Hz}, 1 \mathrm{H}, \boldsymbol{E}$-6fa), 1.33 (ddd, $J=9.6,5.2,2.0 \mathrm{~Hz}, 1 \mathrm{H}, \boldsymbol{Z}-\mathbf{6 f a}$ ).

${ }^{13}$ C NMR (101 MHz, Chloroform-d) for $\boldsymbol{E}$-6fa: $\delta$ 167.15, 142.28, 141.64, 132.30, 130.06, 128.67, 128.60, 126.81, 126.63, 126.29, 118.94, 52.21, 18.23, 16.04 .

${ }^{13}$ C NMR (101 MHz, Chloroform-d) for Z-6fa: $\delta$ 167.10, 141.78, 140.63, 131.49, 129.99, 128.81, 128.47, 126.96, 126.47, 126.36, 119.56, 52.12, 21.78, 13.88 .

IR (ATR): $v\left(\mathrm{~cm}^{-1}\right)$ 3028, 2951, 2849, 1717, 1606, 1453, 1275, 1177, 1109, 868, 697.

HRMS (ESI/QTOF): calcd for $\mathrm{C}_{18} \mathrm{H}_{17} \mathrm{O}_{2}{ }^{+}[\mathrm{M}+\mathrm{H}]^{+}:$265.1223; found: 265.1224 .

$[\boldsymbol{\alpha}]_{\mathbf{D}^{\mathbf{2 0}}}=-310\left(c=1.0, \mathrm{CHCl}_{3}\right)$.

e.r. $(\boldsymbol{Z})$ : $92: 8$ (Chiralpak IB, Hexane $/ \mathrm{IPA}=99.5 / 0.5, \lambda=210 \mathrm{~nm}, 1.00 \mathrm{~mL} / \mathrm{min}, \mathrm{t}_{\mathrm{R}}$ major $=17.0 \mathrm{~min}, \mathrm{t}_{\mathrm{R}}$ minor $=10.0 \mathrm{~min}$ )

Melting point: $54.3-59.1{ }^{\circ} \mathrm{C}$ 

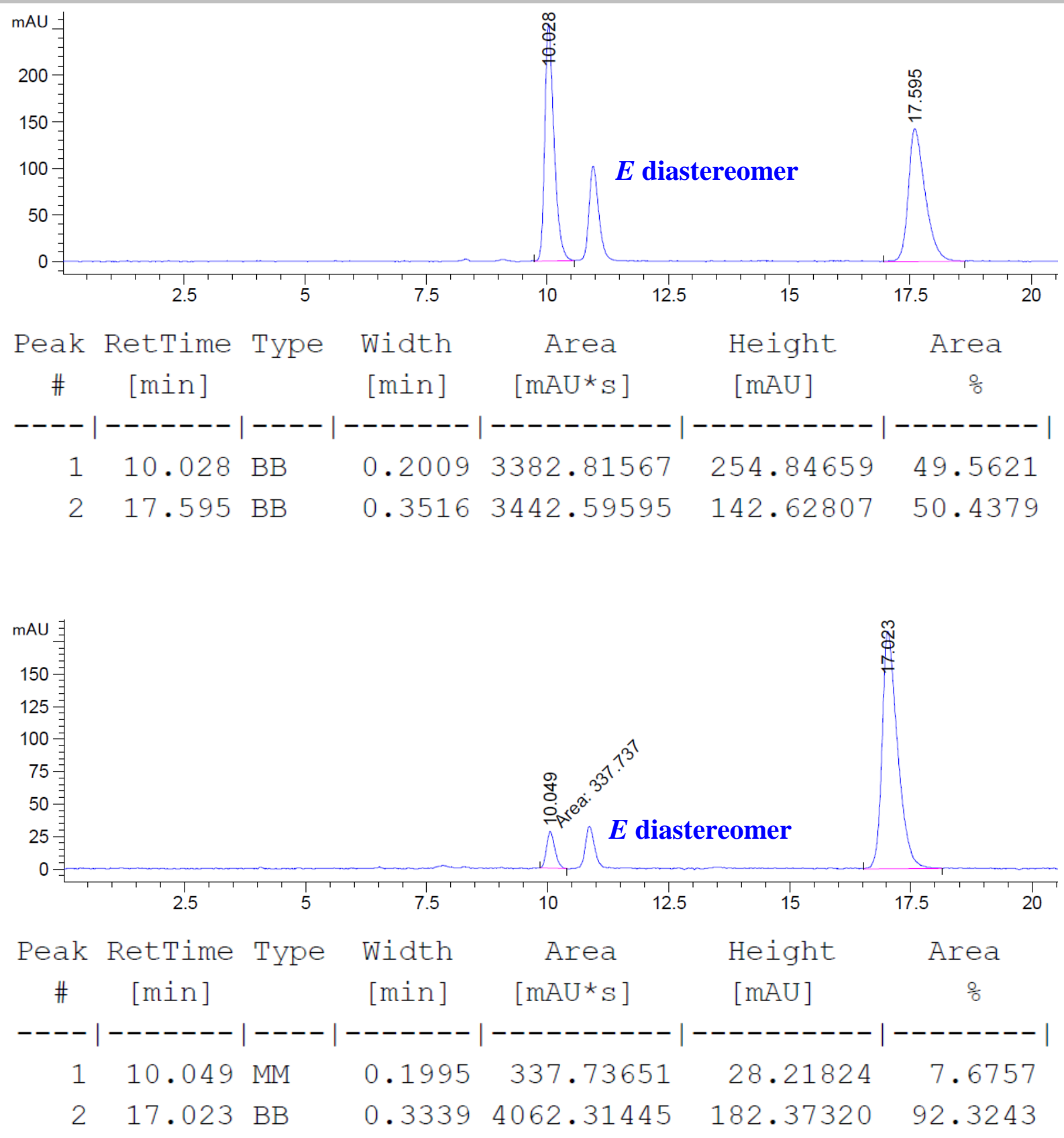


\section{Scale-up reaction (1.00 mmol scale):}

In a $\mathrm{N}_{2}$-filled glovebox, a microwave vial equipped with a magnetic stir bar was charged with $\mathbf{L 9}$ (8.00 mol\%, $0.08 \mathrm{mmol}$ ), $\mathrm{NiCl}_{2}$ (DME) (16.0 mol\%, $0.16 \mathrm{mmol}$ ), Zn powder (1.16 equiv., $1.16 \mathrm{mmol}$ ), 1,3dimethyl-2-imidazolidinone $(0.98 \mathrm{~mL}, 9.00 \mathrm{mmol})$ and CPME $(1.60 \mathrm{~mL})$. The reaction mixture was stirred at room temperature for 15 minutes. A mixture of methyl 4-(2,2-dichlorovinyl)benzoate (1.00 equiv., $1.00 \mathrm{mmol})$, styrene (1.00 equiv., $1.00 \mathrm{mmol})$ and CPME $(3.40 \mathrm{~mL})$ was added to the preformed catalyst. The vial was sealed and the reaction mixture was stirred at $0{ }^{\circ} \mathrm{C}$ for 24 hours. After this time, the reaction mixture was filtered over a plug of $\mathrm{SiO}_{2}$ (eluting with DCM) and concentrated under reduced pressure. The crude residue was purified by silica gel column chromatography (n-pentane:DCM) affording the product in $97 \%$ yield as a mixture of $Z / E$ diastereomers (Z/E: 5.7:1). The enantiomeric ratio for the major $Z$ diastereomer is $92: 8$.

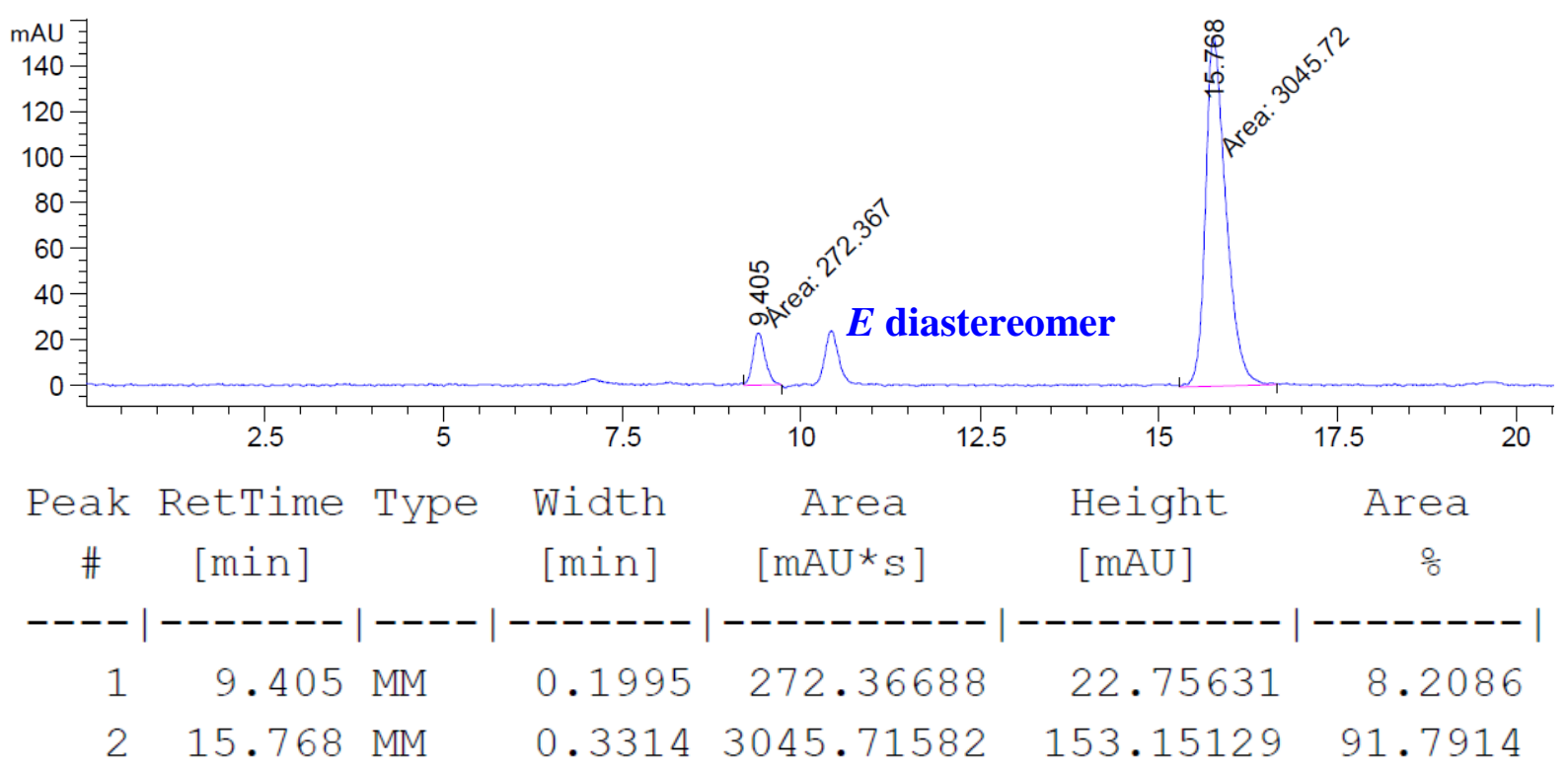




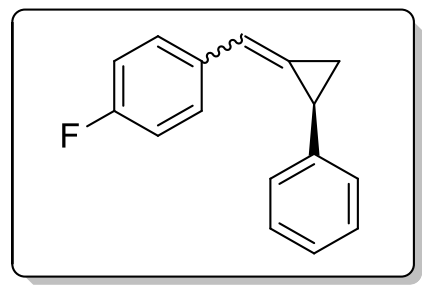

Prepared according to $\underline{\text { GP3 }}$.

Yield: $91 \%$

$\mathbf{R}_{f}$ (100\% pentane): 0.29

Appearance: colourless oil

Z/E ratio: 3.7:1

${ }^{1} \mathbf{H}$ NMR (400 MHz, Chloroform- $\boldsymbol{d}$ ): $\delta 7.60-7.53$ (m, 2H, $\boldsymbol{E}$-6ga), $7.37-7.17$ (m, 5H $\boldsymbol{E}$-6ga $+5 \mathrm{H} \mathrm{Z}$ 6ga), $7.12(\mathrm{~d}, J=7.1 \mathrm{~Hz}, 2 \mathrm{H}, \boldsymbol{Z}$-6ga), $7.07(\mathrm{t}, J=8.7 \mathrm{~Hz}, 2 \mathrm{H}, \boldsymbol{E}$-6ga), $6.97-6.87(\mathrm{~m}, 1 \mathrm{H} \boldsymbol{E}$-6ga $+3 \mathrm{H} \mathrm{Z}$ 6ga), 2.90 (ddd, $J=9.2,4.8,1.9 \mathrm{~Hz}, 1 \mathrm{H}, \boldsymbol{Z}$-6ga), $2.76-2.68$ (ddd, $1 \mathrm{H}, \boldsymbol{E}$-6ga), 2.09 (td, $J=9.2,2.4 \mathrm{~Hz}$, 1H, $\boldsymbol{E}$-6ga), 1.86 (td, $J=9.1,1.9 \mathrm{~Hz}, 1 \mathrm{H}, \boldsymbol{Z}$-6ga), $1.57-1.50$ (m, $1 \mathrm{H}, \boldsymbol{E}$-6ga), 1.28 (m, $1 \mathrm{H}, \boldsymbol{Z}$-6ga).

${ }^{13}$ C NMR (101 MHz, Chloroform-d): $\delta 163.39$ (d, $J=247.5 \mathrm{~Hz}, \boldsymbol{E}$-6ga), 162.03 (d, $J=247.5 \mathrm{~Hz}, \boldsymbol{Z}$ 6ga), 142.05 (E-6ga), 141.06 (Z-6ga), 133.95 (d, $J=3.4$ Hz. $\boldsymbol{E}$-6ga), 133.56 (d, $J=3.2 \mathrm{~Hz}, \boldsymbol{Z}$-6ga), 128.76 (Z-6ga), 128.56 (d, $J=7.9 \mathrm{~Hz}, \boldsymbol{Z}$-6ga), 128.55 (E-6ga), 128.42 (d, $J=7.9 \mathrm{~Hz}, \boldsymbol{E}$-6ga), 128.28 (d, $J=2.7 \mathrm{~Hz}, \boldsymbol{E}$-6ga), 127.25 (d, $J=2.8 \mathrm{~Hz}, \boldsymbol{Z}$-6ga), 126.59, 126.45, 126.21, 126.16, 119.06 (Z-6ga), $118.41(\boldsymbol{E}$-6ga), 115.58 (d, $J=21.2 \mathrm{~Hz}, \boldsymbol{E}$-6ga), 115.50 (d, $J=21.2 \mathrm{~Hz}, \boldsymbol{Z}$-6ga), 21.48 (Z-6ga), 18.11 (E6ga), 15.85 (E-6ga), 13.96 (Z-6ga).

${ }^{19}$ F NMR (376 MHz, Chloroform-d): $\delta$-115.00 (E-6ga), -115.23 (Z-6ga).

IR (ATR): $v\left(\mathrm{~cm}^{-1}\right)$ 3060, 3029, 2968, 2924, 1602, 1506, 1493, 1227, 1156, 852, 822, 696, 512.

HRMS (APPI/LQT-Orbitrap): calcd for $\mathrm{C}_{16} \mathrm{H}_{13} \mathrm{~F}^{+}[\mathrm{M}]^{+}:$224.0996; found: 224.0992.

$[\boldsymbol{\alpha}] \mathbf{D}^{20}=-291\left(c=1.0, \mathrm{CHCl}_{3}\right)$.

e.r. (Z): 91:9 (Reverse Phase HPLC, Chiralpak IB, $\mathrm{H}_{2} \mathrm{O} / \mathrm{ACN}=$ gradient from $60 / 40$ to $40 / 60, \lambda=280$ $\mathrm{nm}, 1.00 \mathrm{~mL} / \mathrm{min}, \mathrm{t}_{\mathrm{R}}$ major $=44.4 \mathrm{~min}, \mathrm{t}_{\mathrm{R}}$ minor $=45.8 \mathrm{~min}$ ) 
WILEY-VCH

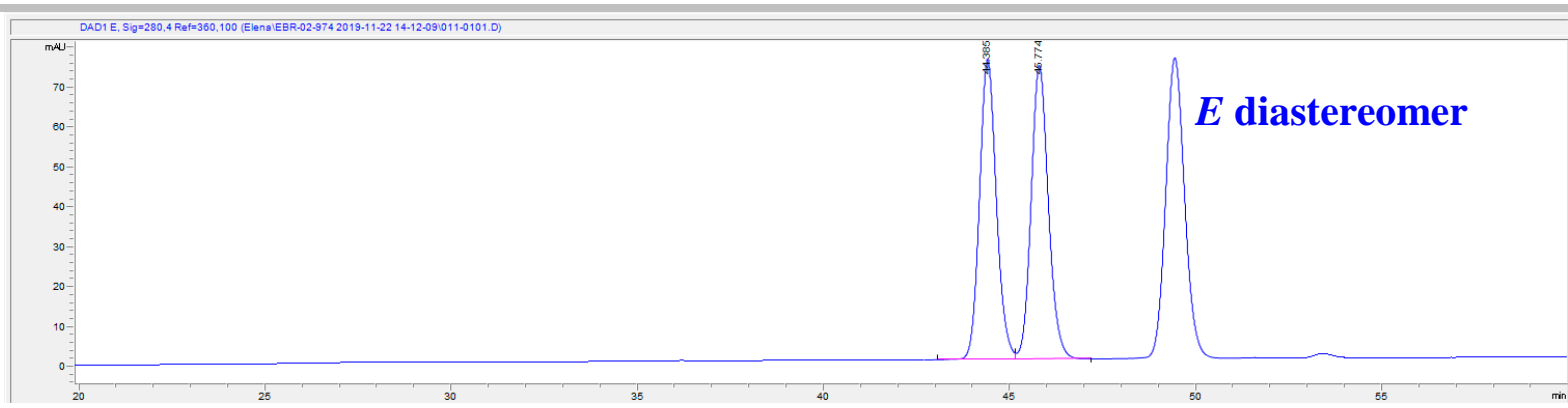

\begin{tabular}{|c|c|c|c|c|c|c|}
\hline$\#$ & Time & Area & Height & Width & Area $\%$ & Symmetry \\
\hline 1 & 44.385 & 2374.1 & 75.4 & 0.4834 & 50.021 & 0.874 \\
\hline 2 & 45.774 & 2372.1 & 73.8 & 0.4912 & 49.979 & 0.858 \\
\hline
\end{tabular}

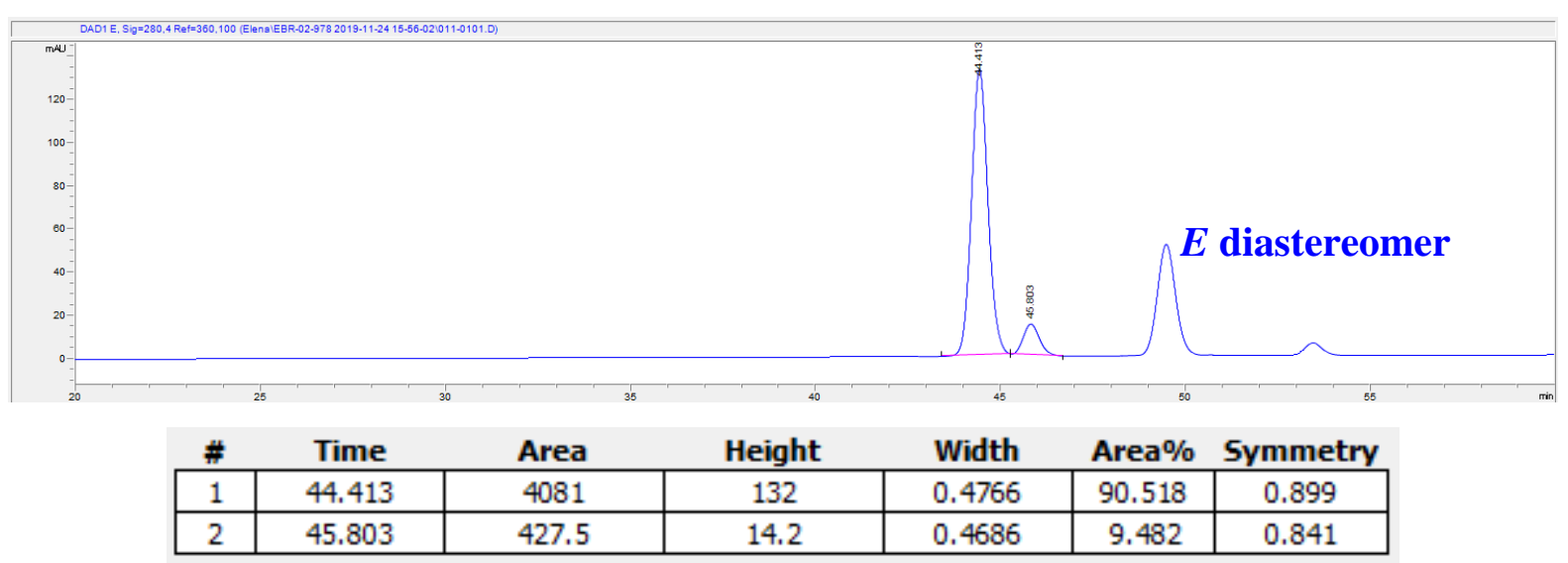




\section{(R)-2-((2-Phenylcyclopropylidene)methyl)naphthalene 6ha}

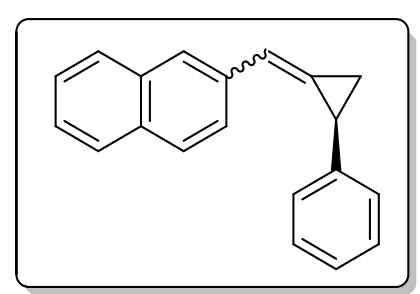

Prepared according to $\underline{\text { GP3 }}$.

Yield: $96 \%$

$\mathbf{R}_{f}(100 \%$ pentane): 0.19

Appearance: light yellow solid

Z/E ratio: $4.1: 1$

${ }^{1}$ H NMR (400 MHz, Chloroform-d): $\delta 8.00-7.89$ (m), $7.86-7.77$ (m), 7.74 (d, $J=8.6 \mathrm{~Hz}, 1 \mathrm{H}, \boldsymbol{Z}$-6ha), 7.67 (dd, $J=8.6,1.6 \mathrm{~Hz}, 1 \mathrm{H}, \boldsymbol{Z}$-6ha), $7.60-7.42$ (m, 2H $\boldsymbol{E}$-6ha $+2 \mathrm{H}$ Z-6ha), $7.41-7.21$ (m), 7.19 (q, $J$ $=2.1 \mathrm{~Hz}, 1 \mathrm{H}, \boldsymbol{Z}$-6ha), 7.16 (q, $J=2.2 \mathrm{~Hz}, 1 \mathrm{H}, \boldsymbol{E}$-6ha), 3.09 (ddd, $J=9.2,4.8,2.0 \mathrm{~Hz}, 1 \mathrm{H}, \boldsymbol{Z}$-6ha), 2.84 (ddd, $J=9.1,5.0,1.7 \mathrm{~Hz}, 1 \mathrm{H}, \boldsymbol{E}$-6ha), $2.28(\mathrm{td}, J=9.2,2.4 \mathrm{~Hz}, 1 \mathrm{H}, \boldsymbol{E}$-6ha) $1.99(\mathrm{td}, J=9.1,2.0 \mathrm{~Hz}, 1 \mathrm{H}$, Z-6ha), 1.73 (ddd, $J=9.3,4.9,2.4 \mathrm{~Hz}, 1 \mathrm{H}, \boldsymbol{E}$-6ha), 1.41 (ddd, $J=9.1,4.9,2.0 \mathrm{~Hz}, 1 \mathrm{H}, \boldsymbol{Z}$-6ha).

${ }^{13}$ C NMR (101 MHz, Chloroform-d): $\delta 142.21$ (E-6ha), 141.32 (Z-6ha), 135.41 (E-6ha), 135.07 (Z6ha), 133.84 (E-6ha), 133.69 (Z-6ha), 132.90 (E-6ha), 132.73 (Z-6ha), 129.40, 128.74, 128.56, 128.47, $128.23,128.08,127.83,127.72,126.64,126.57,126.55,126.44,126.32,126.18,126.13,125.84,125.76$, 124.77, 124.60, 120.40 (Z-6ha), 119.78 (E-6ha), 21.84 (Z-6ha), 18.20 (E-6ha), 16.28 (E-6ha), 13.92 (Z6ha).

IR (ATR): $v\left(\mathrm{~cm}^{-1}\right)$ 3057, 3026, 2965, 2923, 1600, 1507, 1493, 1452, 861, 815, 742, 697, 475.

HRMS (APPI/LTQ-Orbitrap): calcd for $\mathrm{C}_{20} \mathrm{H}_{16}{ }^{+}$[M] ${ }^{+}: 256.1247$; found: 256.1241 .

$[\alpha]^{20}=-280\left(c=1.0, \mathrm{CHCl}_{3}\right)$.

e.r. (Z): 91:9 (Reverse Phase HPLC, Chiralpak IB, $\mathrm{H}_{2} \mathrm{O} / \mathrm{ACN}=$ gradient from 60/40 to 40/60, $\lambda=280$ $\mathrm{nm}, 1.00 \mathrm{~mL} / \mathrm{min}, \mathrm{t}_{\mathrm{R}}$ major $\left.=68.5 \mathrm{~min}, \mathrm{t}_{\mathrm{R}} \operatorname{minor}=73.3 \mathrm{~min}\right)$

Melting point: $85.5-87.7^{\circ} \mathrm{C}$ 

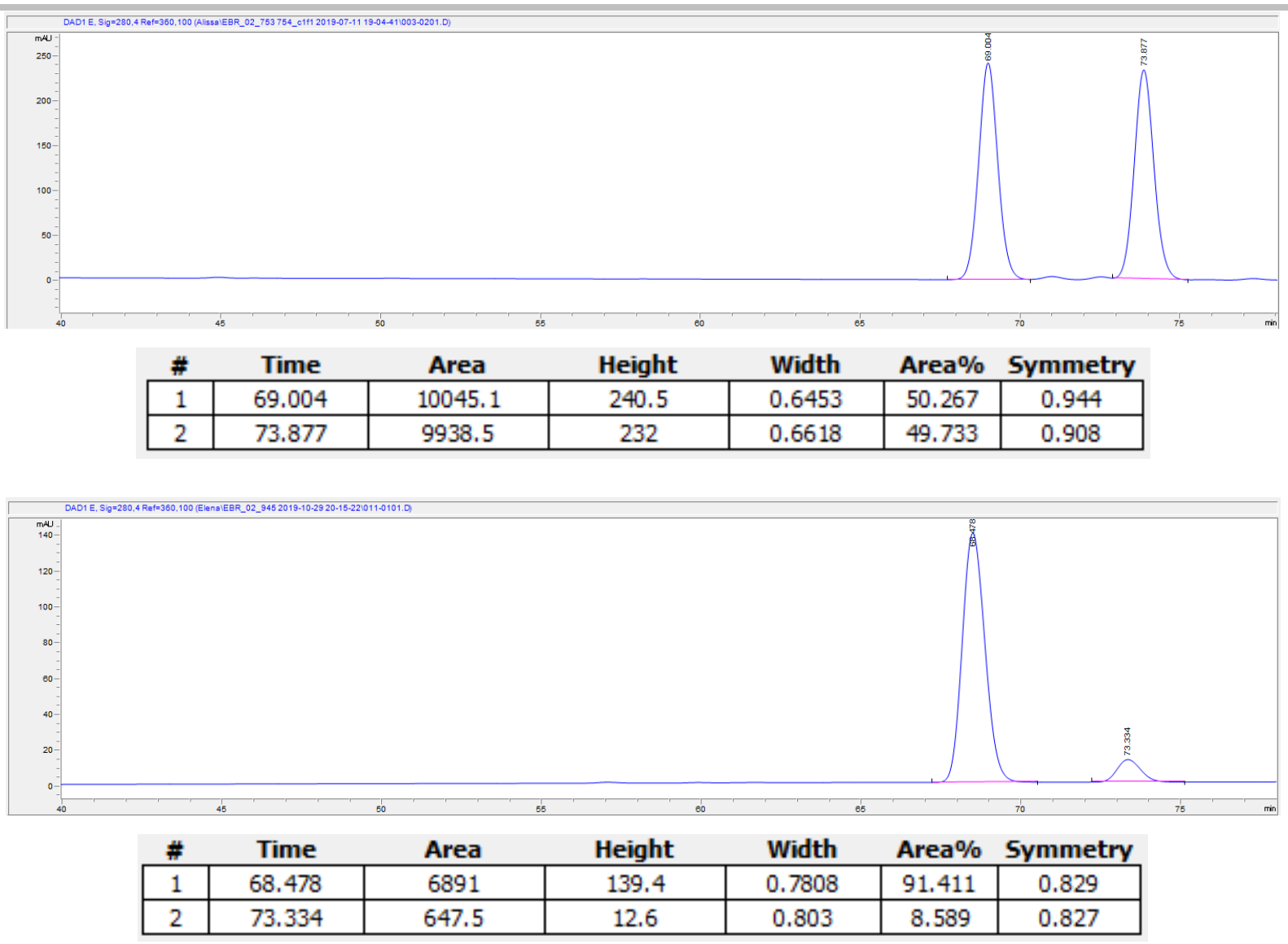

Note: E-diastereomer was not eluted from the column. 


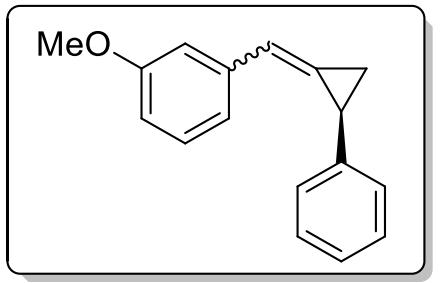

Prepared according to $\underline{\mathbf{G P 3}}$.

Yield: 93\%

$\mathbf{R}_{f}$ (20\% DCM in pentane): 0.21

Appearance: colourless oil

Z/E ratio: 2.0:1

${ }^{1} \mathbf{H}$ NMR (400 MHz, Chloroform-d): $\delta 7.33-7.10$ (m, 8H E-6ia + 6H Z-6ia), 6.96 (d, $J=7.7 \mathrm{~Hz}, 1 \mathrm{H}$, Z-6ia), 6.90 (m, $1 \mathrm{H} \boldsymbol{E}$-6ia + 2H Z-6ia), 6.83 (dd, $J=8.2,2.6,1.0 \mathrm{~Hz}, 1 \mathrm{H}, \boldsymbol{E}$-6ia), 6.72 (dd, $J=8.3,2.6$, $1.0 \mathrm{~Hz}, 1 \mathrm{H}, \boldsymbol{Z}$-6ia), 3.85 (s, 3H, $\boldsymbol{E}$-6ia), 3.53 (s, 3H, Z-6ia), 2.90 (ddd, $J=9.3,4.9,2.1 \mathrm{~Hz}, 1 \mathrm{H}, \boldsymbol{Z}$-6ia), 2.72 (ddd, $J=9.1,5.0,1.7 \mathrm{~Hz}, 1 \mathrm{H}, \boldsymbol{E}$-6ia) $) 2.11$ (td, $J=9.2,2.4 \mathrm{~Hz}, 1 \mathrm{H}, \boldsymbol{E}-6 \mathbf{i a}), 1.89$ (td, $J=9.2,2.0 \mathrm{~Hz}$, 1H, Z-6ia), 1.56 (ddd, $J=7.5,5.0,2.4 \mathrm{~Hz}, 1 \mathrm{H}, \boldsymbol{E}$-6ia), 1.33 (ddd, $J=9.2,4.9,2.0 \mathrm{~Hz}, 1 \mathrm{H}, \boldsymbol{Z}$-6ia).

${ }^{13}$ C NMR (101 MHz, Chloroform-d): $\delta 159.93$ (E-6ia), 159.79 (Z-6ia), 142.13 (E-6ia), 141.28 (Z-6ia), 139.24 (E-6ia), 138.77 (Z-6ia), 129.64, 129.50, 129.18, 128.73, 128.53, 128.24, 126.59, 126.45, 126.15, 126.11, 120.07, 119.92, 119.80, 119.44, 113.82 (Z-6ia), $112.79(\boldsymbol{E}$-6iaX), $112.36(\boldsymbol{E}$-6ia), 111.20 (Z-6ia), 55.36 (E-6ia), 54.98 (Z-6ia), 21.68 (Z-6ia), 18.09 (E-6ia), 16.09 (E-6ia), 13.86 (Z-6ia).

IR (ATR): $v\left(\mathrm{~cm}^{-1}\right)$ 3028, 3001, 2962, 2936, 2835, 1783, 1599, 1492, 1256, 1156, 1002, 697.

HRMS (ESI/QTOF): calcd for $\mathrm{C}_{17} \mathrm{H}_{17} \mathrm{O}^{+}[\mathrm{M}+\mathrm{H}]^{+}:$237.1274; found: 237.1271 .

$[\boldsymbol{\alpha}] \mathbf{D}^{\mathbf{2 0}}=-248\left(c=1.0, \mathrm{CHCl}_{3}\right)$.

e.r. (Z): 94:6 (Reverse Phase HPLC, Chiralpak IB, $\mathrm{H}_{2} \mathrm{O} / \mathrm{ACN}=64 / 36, \lambda=260 \mathrm{~nm}, 0.75 \mathrm{~mL} / \mathrm{min}, \mathrm{t}_{\mathrm{R}}$ major $=104 \mathrm{~min}, \mathrm{t}_{\mathrm{R}} \operatorname{minor}=107 \mathrm{~min}$ ) 

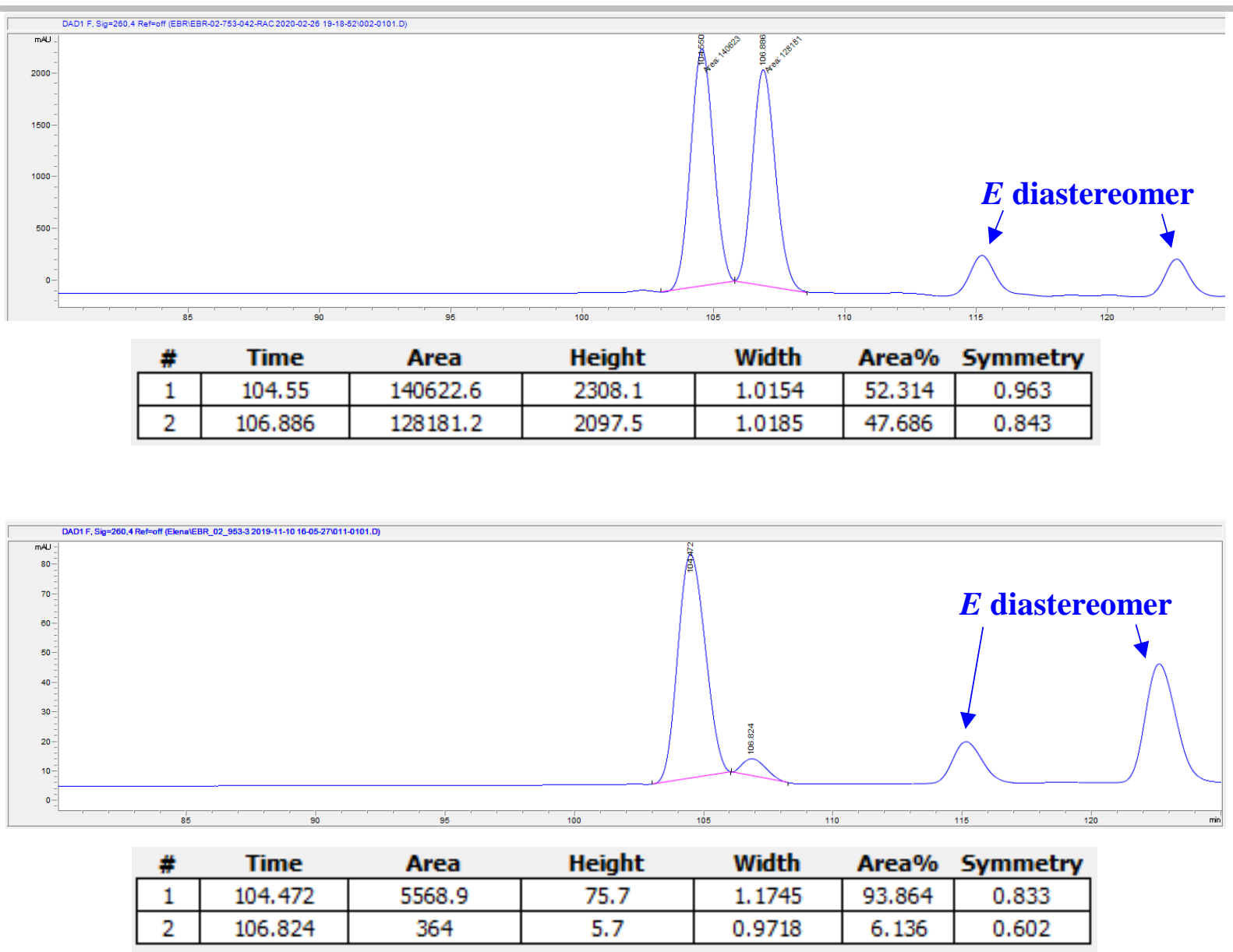


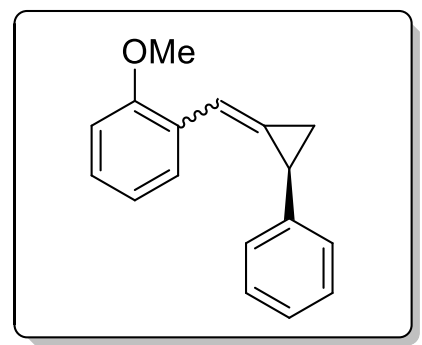

Prepared according to $\underline{\text { GP3 }}$.

Yield: $93 \%$

$\mathbf{R}_{f}$ (20\% DCM in pentane): 0.25

Appearance: colourless oil

Z/E ratio: $4.0: 1$

${ }^{1} \mathbf{H}$ NMR (400 MHz, Chloroform-d): $\delta 7.85$ (d, $\left.J=7.7,1.7 \mathrm{~Hz}, 1 \mathrm{H}, \boldsymbol{E}-\mathbf{6 j a}\right), 7.42(\mathrm{~d}, J=7.7 \mathrm{~Hz}, 1 \mathrm{H}, \boldsymbol{Z}$ 6ja), $7.38(\mathrm{q}, J=2.2 \mathrm{~Hz}, 1 \mathrm{H}, \boldsymbol{Z}$-6ja), $7.34(\mathrm{q}, J=2.3 \mathrm{~Hz}, 1 \mathrm{H}, \boldsymbol{E}$-6ja), $7.30-7.09(\mathrm{~m}, 6 \mathrm{H} \boldsymbol{E}$-6ja $+6 \mathrm{H} \boldsymbol{Z}$ 6ja), 6.99 (t, $J=7.5 \mathrm{~Hz}, 1 \mathrm{H}, \boldsymbol{E}-\mathbf{6 j a}), 6.93(\mathrm{~d}, J=7.8 \mathrm{~Hz}, 1 \mathrm{H}, \boldsymbol{E}-\mathbf{6 j a}), 6.88(\mathrm{~d}, J=8.3,1.1 \mathrm{~Hz}, 1 \mathrm{H}, \boldsymbol{Z}-\mathbf{6 j a}$ ), 6.77 (t, $J=7.5 \mathrm{~Hz}, 1 \mathrm{H}, \boldsymbol{Z}$-6ja), 3.88 (s, 3H, Z-6ja), 3.87 (s, 3H, E-6ja), 2.90 (ddd, $J=9.2,4.7,2.1 \mathrm{~Hz}$, 1H, Z-6ja), 2.71 (ddd, $J=9.1,4.9,1.8 \mathrm{~Hz}, 1 \mathrm{H}, \boldsymbol{E}$-6ja), 2.08 (td, $J=9.2,2.5 \mathrm{~Hz}, 1 \mathrm{H}, \boldsymbol{E}$-6ja), 1.84 (td, $J=$ 9.0, $2.1 \mathrm{~Hz}, 1 \mathrm{H}, \mathbf{Z}-\mathbf{6 j a} \mathbf{a}), 1.58-1.49$ (m, 1H, E-6ja), $1.30-1.22$ (m, 1H, Z-6ja).

${ }^{13}$ C NMR (101 MHz, Chloroform-d): $\delta 156.52$ (E-6ja), 156.24 (Z-6ja), 142.47 (E-6ja), 141.66 (Z-6ja), 128.64, 128.51, 128.46, 128.32, 128.13, 127.50, 127.17, 126.73, 126.65, 126.53, 126.12, 125.98, 125.96, 120.84 (Z-6ja), 120.72 (E-6ja), 113.77 (Z-6ja), 113.44 (E-6ja), 111.07 (E-6ja), 110.86 (Z-6ja), 55.71 (Z-6ja), 55.69 (E-6ja), 21.44 (Z-6ja), 18.12 (E-6ja), 15.89 (E-6ja), 13.94 (Z-6ja).

IR (ATR): $v\left(\mathrm{~cm}^{-1}\right)$ 3028, 2963, 2835, 1598, 1491, 1244, 1051, 749, 697.

HRMS (APPI/LTQ-Orbitrap): calcd for $\mathrm{C}_{17} \mathrm{H}_{16} \mathrm{O}^{+}[\mathrm{M}]^{+}$: 236.1196; found: 236.1190 .

$[\boldsymbol{\alpha}]_{\mathbf{D}^{20}}=-318\left(c=1.0, \mathrm{CHCl}_{3}\right)$.

e.r. (Z): 94:6 (Reverse Phase HPLC, Chiralpak IB, $\mathrm{H}_{2} \mathrm{O} / \mathrm{ACN}=$ gradient from 60/40 to 40/60, $\lambda=254$ $\mathrm{nm}, 1.00 \mathrm{~mL} / \mathrm{min}, \mathrm{t}_{\mathrm{R}}$ major $=48.8 \mathrm{~min}, \mathrm{t}_{\mathrm{R}}$ minor $=53.8 \mathrm{~min}$ ) 


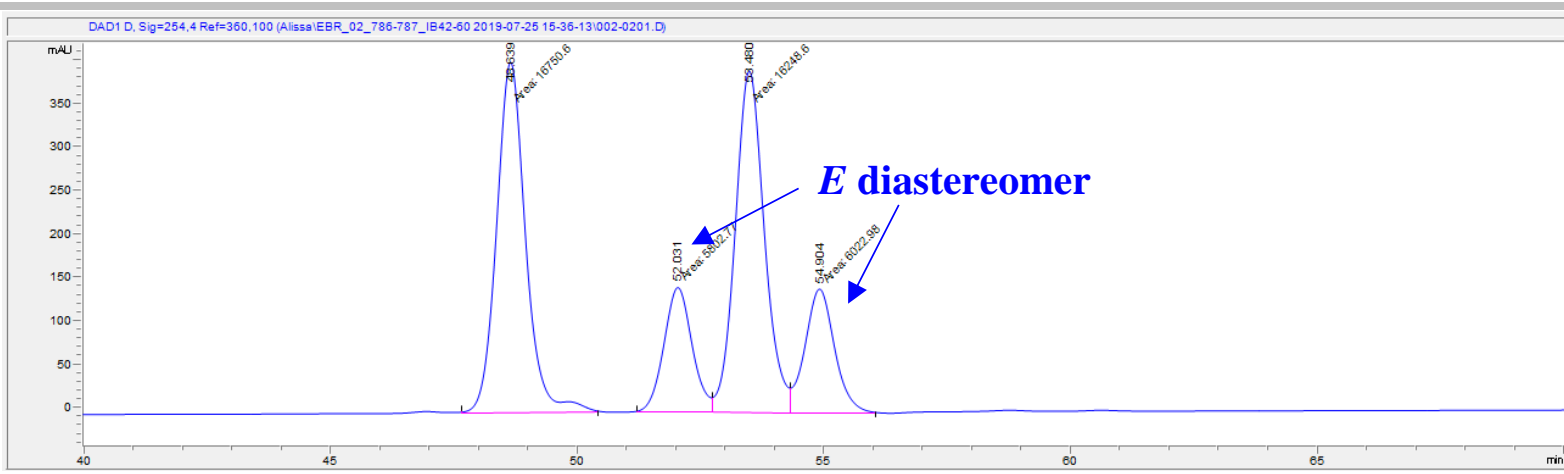

\begin{tabular}{|c|c|c|c|c|c|c|}
\multicolumn{1}{|c}{ \# } & \multicolumn{1}{c}{ Time } & \multicolumn{1}{c}{ Area } & Height & \multicolumn{1}{c|}{ Width } & \multicolumn{1}{c|}{ Area\% $\%$ Symmetry } \\
\hline 1 & 48.639 & 16750.6 & 405.1 & 0.6892 & 37.369 & 0.882 \\
\hline 2 & 52.031 & 5802.8 & 144.2 & 0.6708 & 12.945 & 0 \\
\hline 3 & 53.48 & 16248.6 & 393.8 & 0.6877 & 36.249 & 0.902 \\
\hline 4 & 54.904 & 6023 & 143.2 & 0.7008 & 13.437 & 0.92 \\
\hline
\end{tabular}

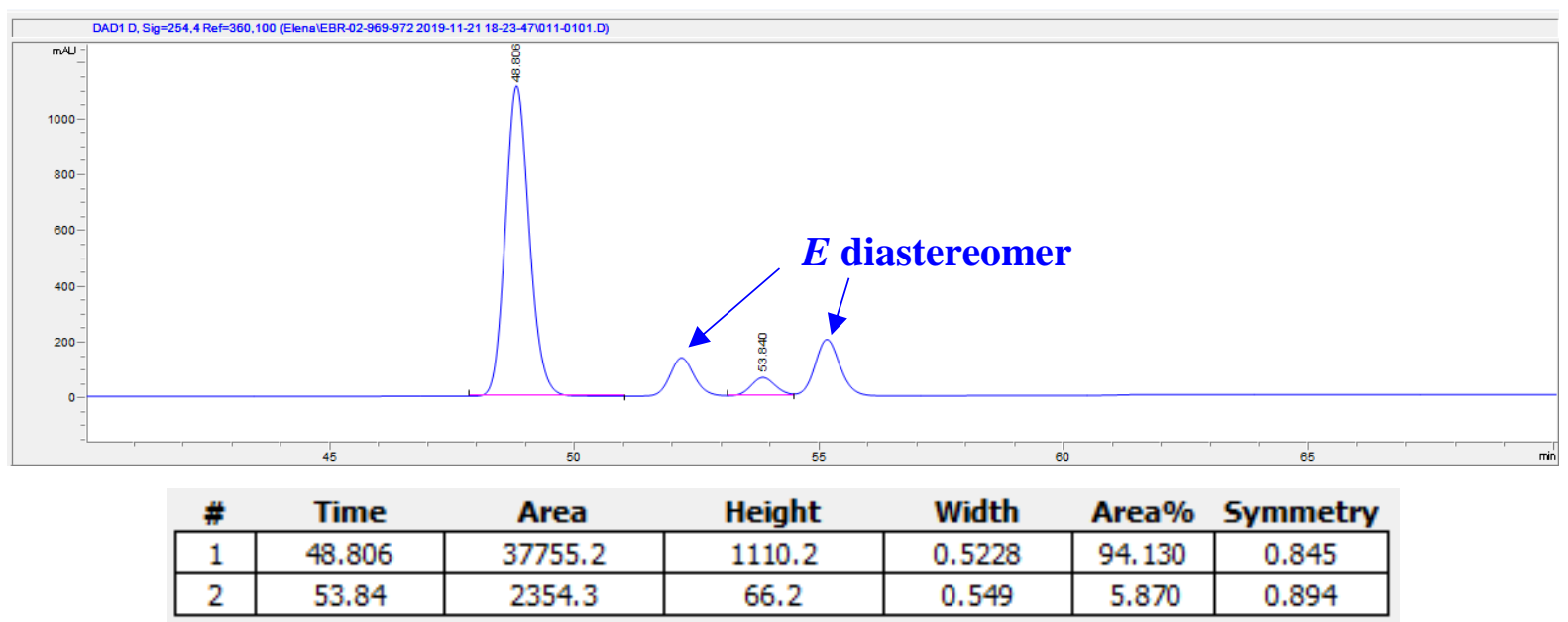




\section{(R)-4-((2-(4-Methoxyphenyl)cyclopropylidene)methyl)tetrahydro-2H-pyran 6kb}

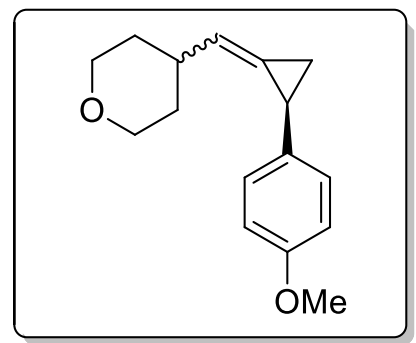

Prepared according to $\underline{\text { GP3 }}$.

Yield: $55 \%$

$\mathbf{R}_{f}(8 \%$ EtOAc in pentane): 0.26

Appearance: yellow oil

Z/E ratio: $12: 1$

${ }^{1}$ H NMR (400 MHz, Chloroform-d): $\delta 7.08-7.03$ (m, 2H, E-6kb), $7.03-6.96$ (m, 2H, Z-6kb), 6.84 6.76 (m, 2H $\boldsymbol{E}-\mathbf{- 6 k b}+2 \mathrm{H}$ Z-6kb), $5.92-5.87$ (m, 1H, $\boldsymbol{E}$-6kb), 5.85 (dq, J = 6.4, $2.0 \mathrm{~Hz}, 1 \mathrm{H}, \boldsymbol{Z}-\mathbf{6 k b}), 3.98$ (m, 2H, E-6kb), 3.94 - 3.85 (m, 2H, Z-6kb), 3.78 (s, 3H $\boldsymbol{E}$-6kb + 3H Z-6kb), 3.48 (tdd, J = 11.5, 5.2, $2.1 \mathrm{~Hz}, 2 \mathrm{H}, \boldsymbol{E}-\mathbf{6 k b}), 3.35$ (tdd, $J=11.6,5.2,2.6 \mathrm{~Hz}, 2 \mathrm{H}, \boldsymbol{Z}-\mathbf{6 k k b}), 2.60-2.52$ (m, 1H, Z-6kb), $2.50-2.46$ (m, 2H, $\boldsymbol{E - 6 k b ) , ~} 2.44$ - 2.30 (m, 1H, Z-6kb), $1.81-1.41$ (m, 5H $\boldsymbol{E}$-6kb + 5H Z-6kb), 1.14 (ddd, J = 8.7, 4.2, 2.0 Hz, 1H, E-6kb), 1.05 (ddd, $J=8.3,4.7,1.7 \mathrm{~Hz}, 1 \mathrm{H}, \mathbf{Z - 6 k b ) . ~}$

${ }^{13}$ C NMR (101 MHz, Chloroform-d): $\delta 157.98$ (E-6kb), 157.87 (Z-6kb), $134.76(Z-6 k b), 134.46(E-$ 6kb), 127.48 (E-6kb), 127.02 (Z-6kb), 125.50 (E-6kb), 125.31 (Z-6kb), 124.01 (Z-6kb), 123.60 (E6kb), 114.00 (Z-6kb), 113.93 (E-6kb), 67.98 (E-6kb), 67.91 (Z-6kb), 55.45 (E-6kb), 55.41 (Z-6kb), 37.94 (Z-6kb), 37.77 (E-6kb), 32.84 (E-6kb), 32.74 (E-6kb), 32.56 (Z-6kb), 32.54 (Z-6kb), 19.86 (Z6kb), 17.89 (E-6kb), 14.28 (E-6kb), 14.21 (Z-6kb).

IR (ATR): $v\left(\mathrm{~cm}^{-1}\right)$ 2954, 2932, 2837, 1512, 1246, 842, 823.

HRMS (APPI/LTQ-Orbitrap): calcd for $\mathrm{C}_{16} \mathrm{H}_{20} \mathrm{O}_{2}{ }^{+}[\mathrm{M}]^{+}:$: 244.1458; found: 244.1457.

$[\boldsymbol{\alpha}] \mathbf{D}^{\mathbf{2 0}}=-142\left(c=1.0, \mathrm{CHCl}_{3}\right)$.

e.r. $(Z): 87: 13$ (Chiralpak IF, Hexane/IPA $=99.5 / 0.5, \lambda=230 \mathrm{~nm}, 1.00 \mathrm{~mL} / \mathrm{min}, \mathrm{t}_{\mathrm{R}}$ major $=14.1 \mathrm{~min}, \mathrm{t}_{\mathrm{R}}$ minor $=12.1 \mathrm{~min})$ 

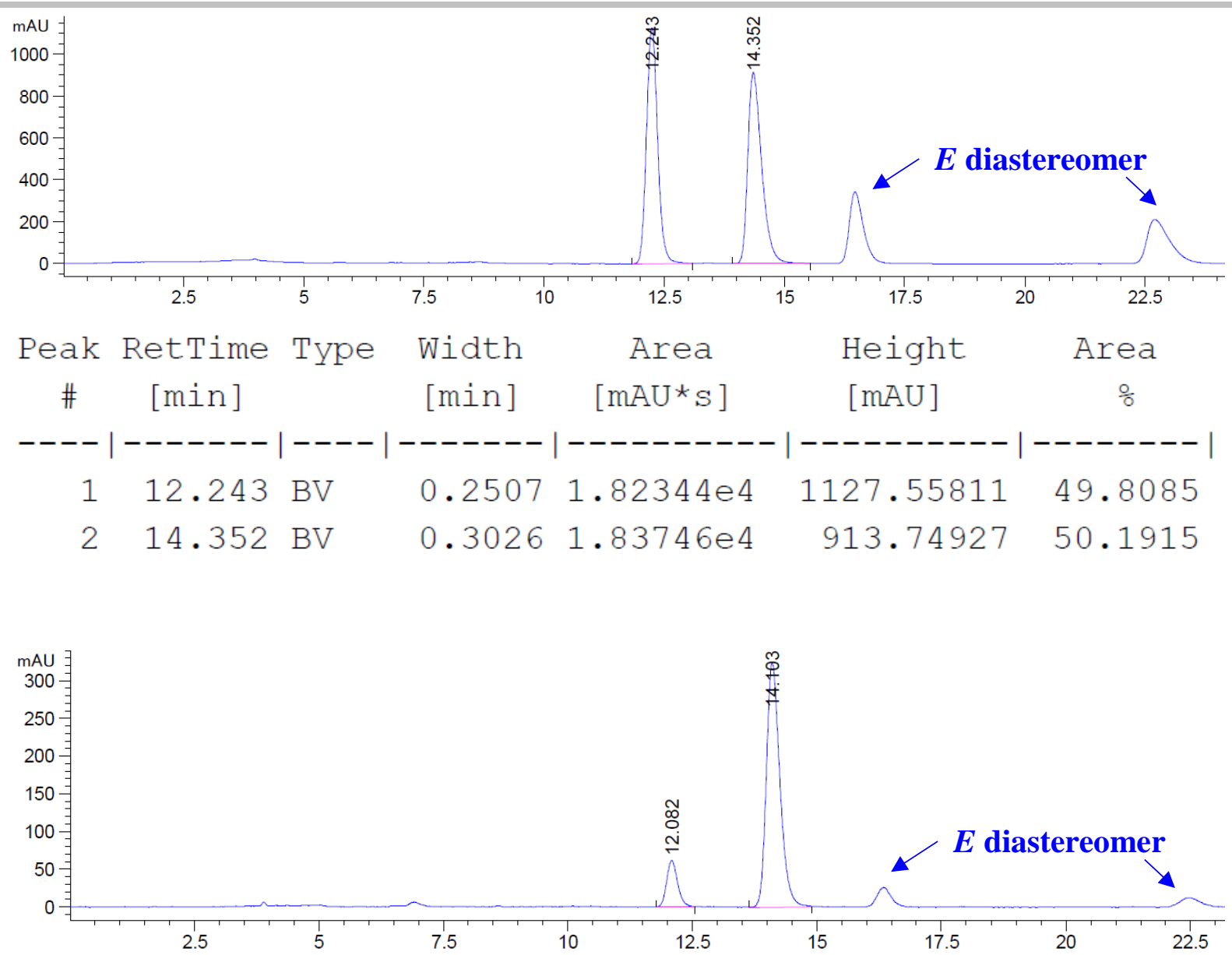

\begin{tabular}{|c|c|c|c|c|c|c|}
\hline $\begin{array}{c}\text { Peak } \\
\#\end{array}$ & $\begin{array}{c}\text { RetTime } \\
\text { [min] }\end{array}$ & Type & $\begin{array}{l}\text { Width } \\
\text { [min] }\end{array}$ & $\begin{array}{c}\text { Area } \\
{\left[\mathrm{mAU}^{*} \mathrm{~S}\right]}\end{array}$ & $\begin{array}{l}\text { Height } \\
{[\mathrm{mAU}]}\end{array}$ & $\begin{array}{c}\text { Area } \\
\quad \frac{\circ}{\circ}\end{array}$ \\
\hline & --- & & & $----------\mid$ & $---------\mid$ & ------ \\
\hline 1 & 12.082 & VB & 0.2297 & 940.31915 & 61.72122 & 13.2745 \\
\hline 2 & 14.103 & VV & 0.2889 & 6143.33789 & 324.47537 & 86.7255 \\
\hline
\end{tabular}




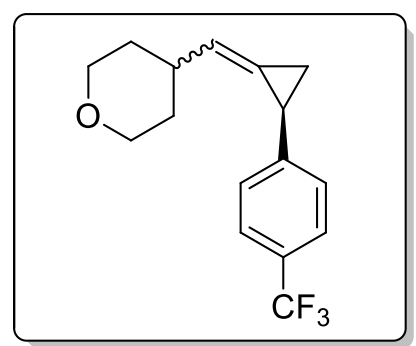

Prepared according to $\underline{\text { GP3 }}$.

Yield: $89 \%$

$\mathbf{R}_{f}$ (50:50 DCM:pentane): 0.36

Appearance: yellow oil

Z/E ratio: $16: 1$

${ }^{1} \mathbf{H}$ NMR (400 MHz, Chloroform- $\left.\boldsymbol{d}\right): \delta 7.50(\mathrm{~m}, 2 \mathrm{H} \boldsymbol{E}$-6kc $+2 \mathrm{H} \boldsymbol{Z}$-6kc), $7.16(\mathrm{~m}, 2 \mathrm{H} \boldsymbol{E}$-6kc $+2 \mathrm{H} \boldsymbol{Z}$ 6kc), 5.97 - 5.85 (m, 1H E-6kc + 1H Z-6kc), $4.03-3.97$ (m, 2H, $\boldsymbol{E - 6 k c ) , ~} 3.95-3.82$ (m, 2H, Z-6kc), $3.53-3.44$ (m, 2H, $\boldsymbol{E}$-6kc), $3.43-3.25$ (m, 2H, Z-6kc), $2.70-2.59$ (m, 1H, Z-6kc), $2.59-2.46(\mathrm{~m}, 1 \mathrm{H}$, $\boldsymbol{E}$-6kc), $2.40-2.27$ (m, $1 \mathrm{H} \boldsymbol{E}$-6kc + 1H Z-6kc), 1.85 (tt, 1H, E-6kc), 1.76 (tt, $J=8.8,1.8 \mathrm{~Hz}, 1 \mathrm{H}, \boldsymbol{Z}$-6kc), $1.61-1.46(\mathrm{~m}, 5 \mathrm{H} \boldsymbol{E}$-6kc $+4 \mathrm{H}$ Z-6kc), $1.22-1.11$ (m, 1H, Z-6kc).

${ }^{13}$ C NMR for Z-6kc (101 MHz, Chloroform-d): $\delta 147.33,128.02$ (q, $\left.J=32.9 \mathrm{~Hz}\right), 126.18,125.46$ (q, $J$ $=4.04 \mathrm{~Hz}), 124.83$ (q, $J=272.7 \mathrm{~Hz}), 124.55,124.49,67.81,37.94,32.42,32.39,20.35,15.19$.

${ }^{19} \mathrm{~F}$ NMR for Z-6kc (376 MHz, Chloroform- $\boldsymbol{d}$ ): $\delta$-62.25.

IR (ATR): $v\left(\mathrm{~cm}^{-1}\right)$ 2933, 2843, 1618, 1323, 1163, 1118, 1068, 1017.

HRMS (APPI/LTQ-Orbitrap): calcd for $\mathrm{C}_{16} \mathrm{H}_{17} \mathrm{~F}_{3} \mathrm{O}^{+}[\mathrm{M}]^{+}:$282.1226; found: 282.1221 .

$[\boldsymbol{\alpha}] \mathbf{D}^{\mathbf{2 0}}=-134\left(c=1.0, \mathrm{CHCl}_{3}\right)$.

e.r. $(Z)$ : 83:17 (Chiralpak ID, Hexane/IPA $=99.5 / 0.5, \lambda=230 \mathrm{~nm}, 1.00 \mathrm{~mL} / \mathrm{min}, \mathrm{t}_{\mathrm{R}}$ major $=6.73 \mathrm{~min}, \mathrm{t}_{\mathrm{R}}$ $\operatorname{minor}=6.25 \mathrm{~min})$ 


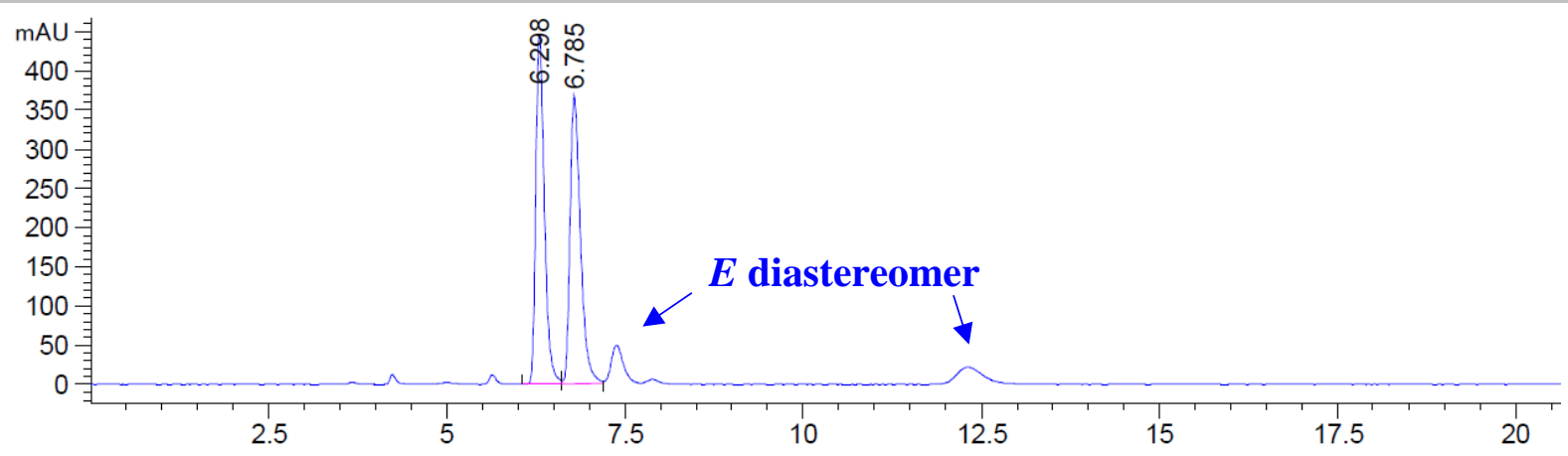

Peak RetTime Type Width Area Height Area

\begin{tabular}{|c|c|c|c|c|c|}
\hline \# & {$[\mathrm{min}]$} & [min] & {$\left[\mathrm{mAU}^{*} \mathrm{~s}\right]$} & {$[\mathrm{mAU}]$} & 응 \\
\hline-7 & $-----\mid---$ & ------ & ---------- & ---------- & -------- \\
\hline 1 & $6.298 \mathrm{BV}$ & 0.1303 & 3758.46753 & 446.19244 & 49.9827 \\
\hline 2 & $6.785 \mathrm{VV}$ & 0.1575 & 3761.06323 & 367.10974 & 50.0173 \\
\hline
\end{tabular}

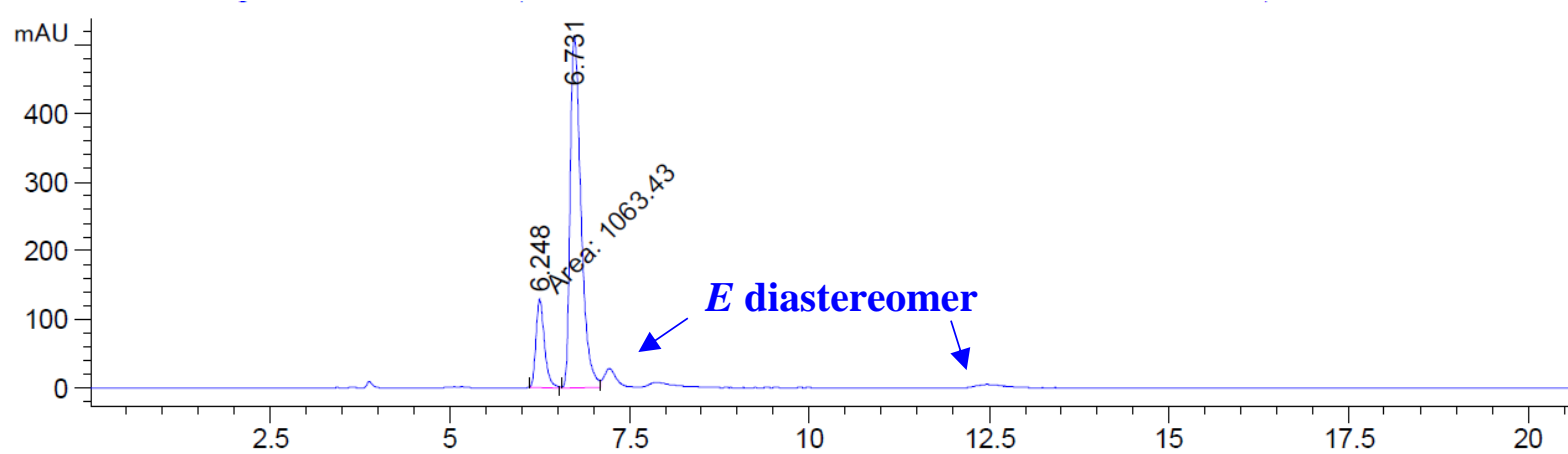

Peak RetTime Type Width Area Height Area

\begin{tabular}{|c|c|c|c|c|c|}
\hline \# & {$[\mathrm{min}]$} & [min] & {$\left[\mathrm{mAU}^{*} \mathrm{~s}\right]$} & {$[\mathrm{mAU}]$} & 응 \\
\hline & $-----\mid---$ & 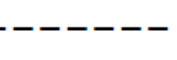 & $1----------$ & $----------\mid$ & -------- \\
\hline 1 & $6.248 \mathrm{MM}$ & 0.1372 & 1063.42725 & 129.17557 & 16.5350 \\
\hline 2 & $6.731 \mathrm{VV}$ & 0.1601 & 5367.92920 & 512.83093 & 83.4650 \\
\hline
\end{tabular}




\section{(R)-2-(2-(4-Methoxybenzylidene)cyclopropyl)naphthalene 6ad}

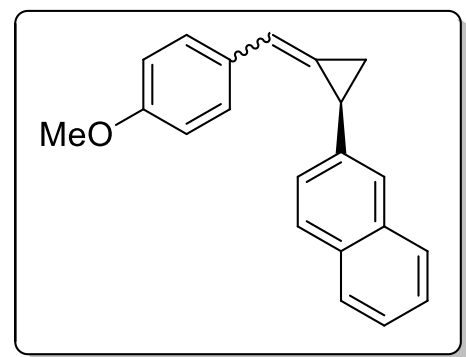

Prepared according to $\underline{\text { GP3 }}$.

Yield: 99\%

$\mathbf{R}_{f}$ (20\% DCM in pentane): 0.23

Appearance: white solid

Z/E ratio: $2.0: 1$

${ }^{1}$ H NMR (400 MHz, Chloroform-d): $\delta 7.84-7.71$ (m, 4H E-6ad + 3H Z-6ad), 7.64 (d, $J=12.6 \mathrm{~Hz}$, 1H, Z-6ad), 7.58 (d, $J=8.7 \mathrm{~Hz}, 2 \mathrm{H}, \boldsymbol{E}$-6ad), $7.49-7.37$ (m, 4H $\boldsymbol{E}$-6ad + 1H Z-6ad), $7.37-7.31$ (m, 2H, Z-6ad), 7.29 (dd, $J=8.5,1.8 \mathrm{~Hz}, 1 \mathrm{H}, \boldsymbol{E}$-6ad), 7.21 (dd, $J=8.5,1.8 \mathrm{~Hz}, 1 \mathrm{H}, \boldsymbol{Z}$-6ad), $6.99-6.89$ (m, $1 \mathrm{H}$ $\boldsymbol{E}$-6ad + 2H Z-6ad), 6.80 - 6.70 (d, 2H, m, 2H Z-6ad), 3.85 (s, 3H, E-6ad), 3.73 (s, 3H, Z-6ad), 3.04 (ddd, $J=9.0,4.6,2.0 \mathrm{~Hz}, 1 \mathrm{H}, \boldsymbol{Z}$-6ad), 2.86 (ddd, $J=8.8,4.8,1.6 \mathrm{~Hz}, 1 \mathrm{H}, \boldsymbol{E}$-6ad), 2.15 (td, $J=9.0,2.4$ $\mathrm{Hz}, 1 \mathrm{H}, \boldsymbol{E}$-6ad), 1.91 (td, $J=8.9,2.0 \mathrm{~Hz}, 1 \mathrm{H}, \boldsymbol{Z}$-6ad), 1.61 (ddd, $J=9.2,4.7,2.4 \mathrm{~Hz}, 1 \mathrm{H}, \boldsymbol{E}$-6ad), 1.35 (ddd, $J=8.9,4.6,2.0 \mathrm{~Hz}, 1 \mathrm{H}, \mathbf{Z}$-6ad).

${ }^{13}$ C NMR (101 MHz, Chloroform-d): $\delta 159.08$ (E-6ad), 158.86 (Z-6ad), 139.97 (E-6ad), 139.23 (Z6ad), 133.80 (Z-6ad), 133.66 (E-6ad), 132.29, 132.24, 130.69, 130.32, 128.43, 128.31, 128.20, 128.15, 127.77, 127.75, 127.53, 127.46, 126.22, 126.16, 126.11, 125.25, 125.16, 125.01, 124.96, 124.81, 124.77, 119.84 (Z-6ad), 119.16 (E-6ad), 114.19 (E-6ad), 114.10 (Z-6ad), 55.48 (E-6ad), 55.33 (Z-6ad), 21.75 (Z-6ad), 18.33 (E-6ad), 16.16 (E-6ad), 14.16 (Z-6ad).

IR (ATR): $v\left(\mathrm{~cm}^{-1}\right)$ 2959, 2921, 2850, 2835, 1604, 1509, 1246, 1172, 1032, 846, 812, 760, 477.

HRMS (ESI/QTOF): calcd for $\mathrm{C}_{21} \mathrm{H}_{19} \mathrm{O}^{+}[\mathrm{M}+\mathrm{H}]^{+}$: 287.1430; found: 287.1429 .

$[\boldsymbol{\alpha}] \mathbf{D}^{\mathbf{2 0}}=-351\left(c=1.0, \mathrm{CHCl}_{3}\right)$.

e.r. (Z): 91:9 (Reverse Phase HPLC, Chiralpak IB, $\mathrm{H}_{2} \mathrm{O} / \mathrm{ACN}=$ gradient from 50/50 to 40/60, $\lambda=280$ $\mathrm{nm}, 1.00 \mathrm{~mL} / \mathrm{min}, \mathrm{t}_{\mathrm{R}}$ major $=64.9 \mathrm{~min}, \mathrm{t}_{\mathrm{R}}$ minor $=59.5 \mathrm{~min}$ )

Melting point: $66.2-68.6^{\circ} \mathrm{C}$ 

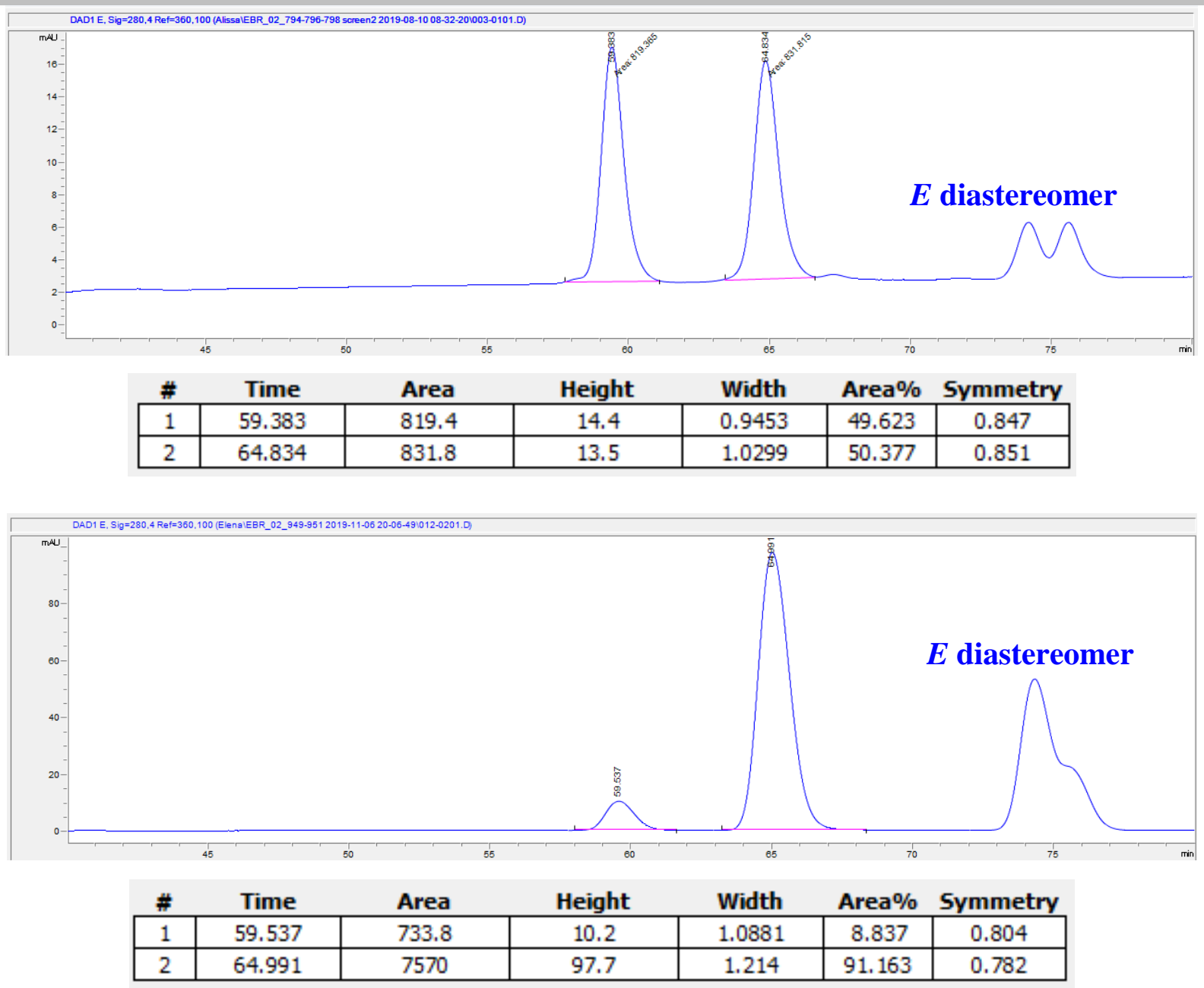


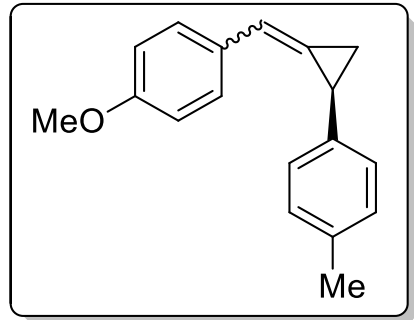

Prepared according to $\underline{\mathbf{G P 3}}$.

Yield: $91 \%$

$\mathbf{R}_{f}(20 \%$ DCM in pentane): 0.46

Appearance: colourless oil

Z/E ratio: $2.5: 1$

${ }^{1}$ H NMR (400 MHz, Chloroform-d): $\delta 7.54$ (d, $J=8.7 \mathrm{~Hz}, 2 \mathrm{H}, \boldsymbol{E}$-6ae), 7.33 (d, $J=8.8 \mathrm{~Hz}, 2 \mathrm{H}, \boldsymbol{Z}$-6ae), 7.12 - 7.01 (m, 4H $\boldsymbol{E}$-6ae + 4H Z-6ae), 6.92 (d, $J=8.8$ Hz, 2H, $\boldsymbol{E}$-6ae), 6.88 (q, 1H, Z-6ae), 6.86 (q, 1H, E-6ae), 6.80 (d, J = 8.7 Hz, 2H, Z-6ae), 3.85 (s, 3H, E-6ae), 3.77 (s, 3H, Z-6ae), 2.87 (ddd, J = 9.0, 4.6, $2.0 \mathrm{~Hz}, 1 \mathrm{H}, \boldsymbol{Z}$-6ae), 2.67 (ddd, $J=8.8,4.8,1.7 \mathrm{~Hz}, 1 \mathrm{H}, \boldsymbol{E}$-6ae), 2.33 (s, 3H, E-6ae), 2.32 (s, 3H, Z-6ae), $2.05(\mathrm{td}, J=9.0,2.4 \mathrm{~Hz}, 1 \mathrm{H}, \boldsymbol{E}$-6ae), $1.81(\mathrm{td}, J=8.9,2.0 \mathrm{~Hz}, 1 \mathrm{H}, \boldsymbol{Z}-\mathbf{6 a e}), 1.48(\mathrm{ddd}, J=9.1,4.8,2.4 \mathrm{~Hz}$, $1 \mathrm{H}, \boldsymbol{E}$-6ae), 1.20 (ddd, $J=8.8,4.7,2.0 \mathrm{~Hz}, 1 \mathrm{H}, \boldsymbol{Z}$-6ae).

${ }^{13}$ C NMR (101 MHz, Chloroform-d): $\delta 158.95$ (E-6ae), 158.77 (Z-6ae), 139.34 (E-6ae), 138.38 (Z6ae), 135.55 (E-6ae), 135.53 (Z-6ae), 130.79, 130.44, 129.39, 129.18, 128.28, 128.10, 126.49, 126.46, 126.42, 125.25, 119.52 (Z-6ae), 118.73 (E-6ae), 114.11 (E-6ae), 114.05 (Z-6ae), 55.45 (E-6ae), 55.34 (Z-6ae), 21.19 (Z-6ae), 21.15 (Z/E-6ae), 17.71 (E-6ae), 15.81 (E-6ae), 13.86 (Z-6ae).

IR (ATR): $v\left(\mathrm{~cm}^{-1}\right)$ 2999, 2961, 2921, 2834, 1606, 1509, 1462, 1289, 1244, 1172, 1111, 804, 529.

HRMS (APPI/LTQ-Orbitrap): calcd for $\mathrm{C}_{18} \mathrm{H}_{18} \mathrm{O}^{+}[\mathrm{M}]^{+}:$250.1352; found: 250.1352.

$[\boldsymbol{\alpha}]_{\mathbf{D}^{\mathbf{2 0}}}=-380\left(c=1.0, \mathrm{CHCl}_{3}\right)$.

e.r. $(\boldsymbol{Z})$ : 92:8 (Chiralpak IG, Hexane/IPA $=99.5 / 0.5, \lambda=254 \mathrm{~nm}, 1.00 \mathrm{~mL} / \mathrm{min}, \mathrm{t}_{\mathrm{R}}$ major $=7.59 \mathrm{~min}, \mathrm{t}_{\mathrm{R}}$ minor $=8.29 \mathrm{~min})$ 

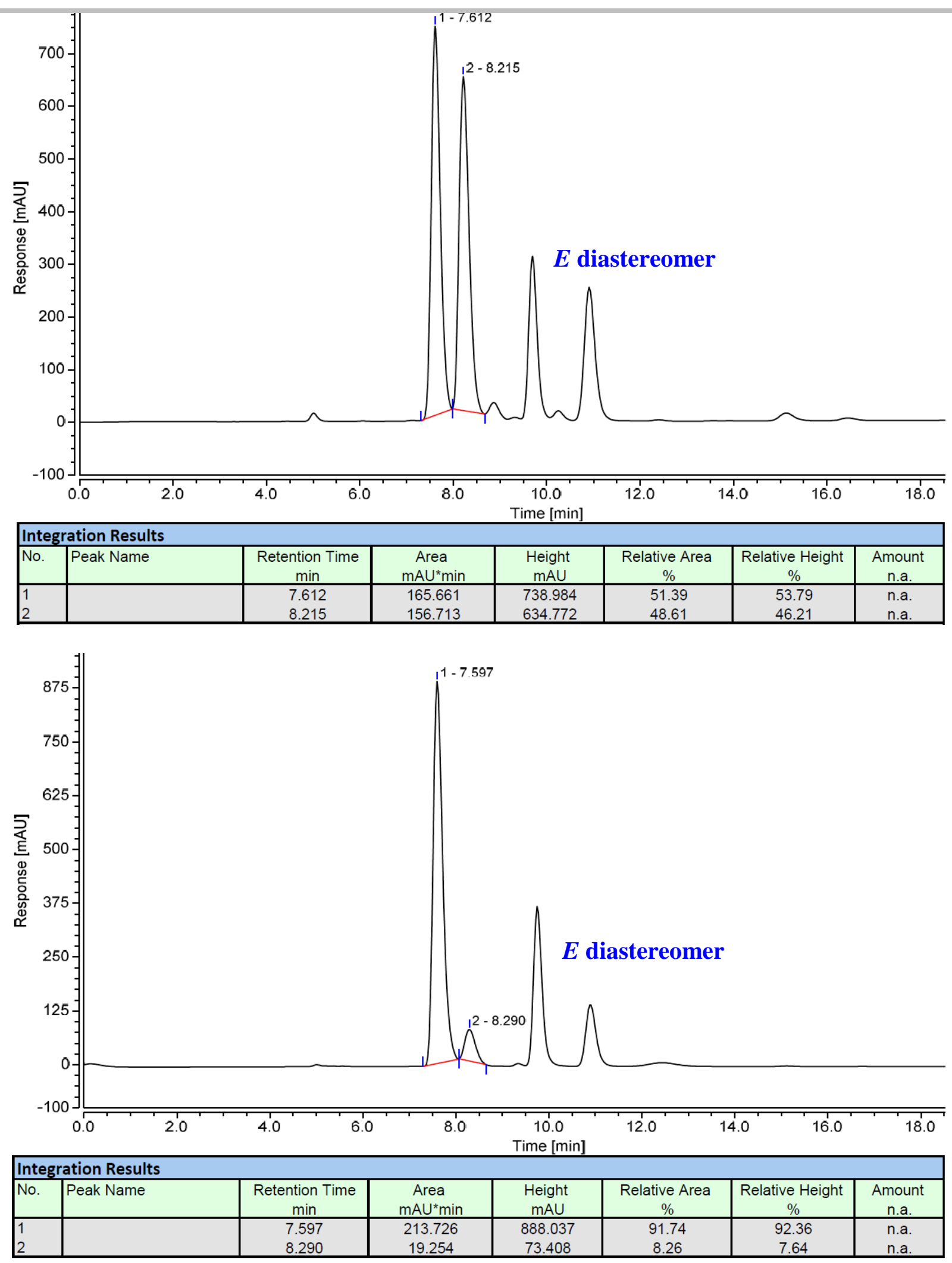


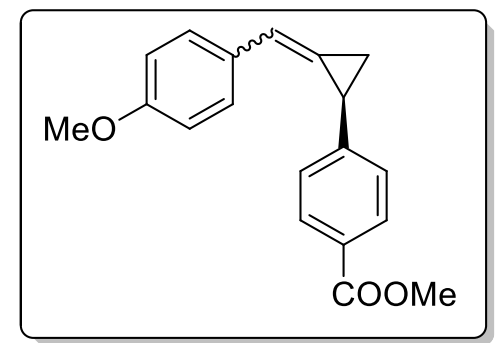

Prepared according to $\underline{\mathbf{G P 3}}$.

Yield: $71 \%$

$\mathbf{R}_{f}$ (50\% DCM in pentane): 0.25

Appearance: yellow solid

Z/E ratio: 3.0:1

${ }^{1}$ H NMR (400 MHz, Chloroform-d): $\delta 7.92(\mathrm{~m}, 2 \mathrm{H} \boldsymbol{E}$-6af + 2H Z-6af), $7.53(\mathrm{~d}, J=8.6 \mathrm{~Hz}, 2 \mathrm{H}, \boldsymbol{E}$-6af), $7.30-7.14$ (m, 2H $\boldsymbol{E}$-6af + 4H Z-6af), $6.92(\mathrm{~d}, J=8.7 \mathrm{~Hz}, 2 \mathrm{H}, \boldsymbol{E}$-6af), 6.89 (q, $J=2.1 \mathrm{~Hz}, 1 \mathrm{H}, \boldsymbol{Z}$-6af), 6.85 (q, $J=2.2 \mathrm{~Hz}, 1 \mathrm{H}, \boldsymbol{E}$-6af), 6.76 (d, $J=8.8 \mathrm{~Hz}, 2 \mathrm{H}, \boldsymbol{Z}$-6af), 3.89 (s, 3H, $\boldsymbol{E}$-6af), 3.88 (s, 3H, Z-6af), 3.84 (s, 3H, $\boldsymbol{E}$-6af), 3.75 (s, 3H, Z-6af), 2.91 (ddd, $J=9.0,4.5,2.0 \mathrm{~Hz}, 1 \mathrm{H}, \boldsymbol{Z}$-6af), 2.72 (ddd, $J=8.9$, 4.7, $1.7 \mathrm{~Hz}, 1 \mathrm{H}, \boldsymbol{E}$-6af), 2.15 (td, $J=9.1,2.5 \mathrm{~Hz}, 1 \mathrm{H}, \boldsymbol{E}$-6af), 1.91 (td, $J=8.9,2.0 \mathrm{~Hz}, 1 \mathrm{H}, \boldsymbol{Z}$-6af), 1.55 (ddd, 1H, E-6af), 1.31 (ddd, $J=9.0,4.5,2.0 \mathrm{~Hz}, 1 \mathrm{H}, \boldsymbol{Z}$-6af).

${ }^{13}$ C NMR (101 MHz, Chloroform-d): $\delta$ 167.19, 159.19 (E-6af), 159.01 (Z-6af), 148.40 (E-6af), 147.37 (Z-6af), 130.31, 130.08, 129.94, 129.85, 128.25, 127.95, 127.84, 126.40, 126.36, 125.62, 124.39, 119.81 (Z-6af), 119.37 (E-6af), 114.20 (E-6af), 114.11 (Z-6af), 55.47 (E-6af), 55.35 (Z-6af), 52.10, 21.55 (Z6af), 18.29 (E-6af), 16.76 (E-6af), 14.55 (Z-6af).

IR (ATR): $v\left(\mathrm{~cm}^{-1}\right)$ 2952, 2837, 1717, 1608, 1510, 1435, 1277, 1248, 1175, 1110, 1032, 847, 702.

HRMS (ESI/QTOF): calcd for $\mathrm{C}_{19} \mathrm{H}_{19} \mathrm{O}_{3}{ }^{+}[\mathrm{M}+\mathrm{H}]^{+}:$295.1329; found: 295.1330 .

$[\boldsymbol{\alpha}] \mathbf{D}^{\mathbf{2 0}}=-349\left(c=1.0, \mathrm{CHCl}_{3}\right)$.

e.r. $(Z): 89: 11$ (Chiralpak IA, Hexane/IPA $=95 / 5, \lambda=254 \mathrm{~nm}, 1.00 \mathrm{~mL} / \mathrm{min}, \mathrm{t}_{\mathrm{R}}$ major $=8.07 \mathrm{~min}, \mathrm{t}_{\mathrm{R}}$ minor $=9.26 \mathrm{~min}$ )

Melting point: $74.6-76.1{ }^{\circ} \mathrm{C}$ 

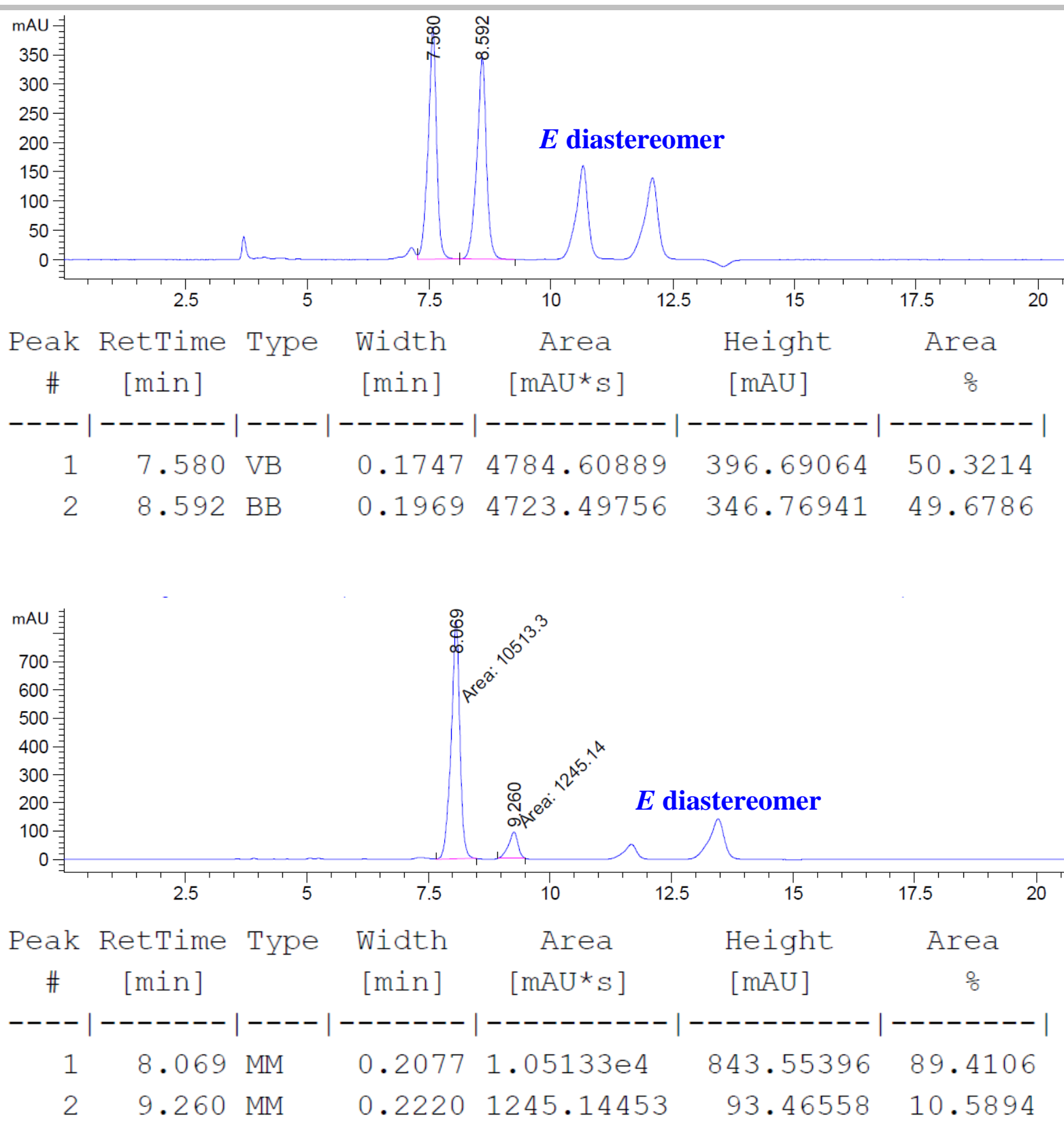


\section{(R)-1-Chloro-4-(2-(4-methoxybenzylidene)cyclopropyl)benzene 6ag}

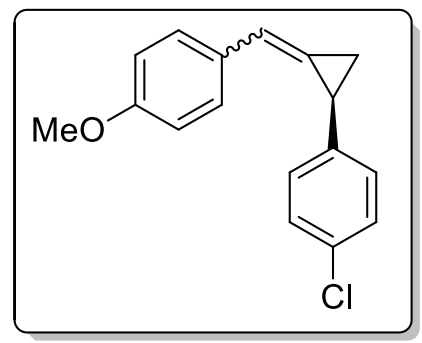

Prepared according to $\underline{\mathbf{G P 3}}$.

Yield: $90 \%$

$\mathbf{R}_{f}$ (20\% DCM in pentane): 0.19

Appearance: colourless oil

Z/E ratio: $3.4: 1$

${ }^{1} \mathbf{H}$ NMR (400 MHz, Chloroform-d): $\delta 7.53(\mathrm{~d}, J=8.7 \mathrm{~Hz}, 2 \mathrm{H}, \boldsymbol{E}-\mathbf{6 a g}), 7.32-7.18(\mathrm{~m}, 2 \mathrm{H} \boldsymbol{E}-\mathbf{6 a g}+4 \mathrm{H}$ Z-6ag), 7.10 (d, $J=8.4 \mathrm{~Hz}, 2 \mathrm{H}, \boldsymbol{E}$-6ag), 7.04 (d, $J=8.4 \mathrm{~Hz}, 2 \mathrm{H}, \boldsymbol{Z}$-6ag), 6.92 (d, $J=8.7 \mathrm{~Hz}, 2 \mathrm{H}, \boldsymbol{E}$-6ag), 6.88 (q, 1H, Z-6ag), 6.85 (q, 1H, E-6ag), 6.79 (d, $J=8.8 \mathrm{~Hz}, 2 \mathrm{H}, \boldsymbol{Z}$-6ag), 3.84 (s, 3H, E-6ag), 3.77 (s, 3H, Z-6ag), 2.85 (ddd, $J=9.0,4.5,2.0 \mathrm{~Hz}, 1 \mathrm{H}, \boldsymbol{Z}$-6ag), 2.65 (ddd, $J=8.9,4.7,1.7 \mathrm{~Hz}, 1 \mathrm{H}, \boldsymbol{E}$-6ag), 2.08 (td, $J=9.0,2.4 \mathrm{~Hz}, 1 \mathrm{H}, \boldsymbol{E}-\mathbf{6 a g}), 1.84(\mathrm{td}, J=8.9,2.0 \mathrm{~Hz}, 1 \mathrm{H}, \boldsymbol{Z}-\mathbf{6 a g}), 1.47$ (ddd, $J=9.3,4.6,2.4 \mathrm{~Hz}, 1 \mathrm{H}$, E-6ag), 1.21 (ddd, $J=9.0,4.5,2.0 \mathrm{~Hz}, 1 \mathrm{H}, \boldsymbol{Z}$-6ag).

${ }^{13}$ C NMR (101 MHz, Chloroform-d): $\delta 159.11$ (E-6ag), 158.95 (Z-6ag), 141.02 (E-6ag), 140.05 (Z6ag), 131.64 (Z-6ag), 131.59 (E-6ag), 130.43 (E-6ag), 130.07 (Z-6ag), 128.81(Z-6ag), 128.56 (E-6ag), 128.27 (Z-6ag), 128.18, (E-6ag) 127.91 (E-6ag), 127.85 (Z-6ag), 125.62 (E-6ag), 124.37 (Z-6ag), 119.87 (Z-6ag), 119.24 (E-6ag), 114.16 (E-6ag), 114.11 (Z-6ag), 55.47 (E-6ag), 55.36 (Z-6ag), 20.84 (Z-6ag), 17.50 (E-6ag), 16.17 (E-6ag), 14.13 (Z-6ag).

IR (ATR): $v\left(\mathrm{~cm}^{-1}\right)$ 3032, 2961, 2932, 2907, 2835, 1606, 1510, 1491, 1245, 1172, 1033, 832, 525.

HRMS (APPI/LTQ-Orbitrap): calcd for $\mathrm{C}_{17} \mathrm{H}_{15} \mathrm{ClO}^{+}[\mathrm{M}]^{+}:$: 270.0806; found: 270.0806 .

$[\alpha]_{\mathbf{D}^{20}}=-410\left(c=1.0, \mathrm{CHCl}_{3}\right)$.

e.r. (Z): 89:11 (Chiralpak IG, Hexane/IPA $=99.5 / 0.5, \lambda=254 \mathrm{~nm}, 1.00 \mathrm{~mL} / \mathrm{min}, \mathrm{t}_{\mathrm{R}}$ major $=7.21 \mathrm{~min}, \mathrm{t}_{\mathrm{R}}$ minor $=8.28 \mathrm{~min}$ ) 

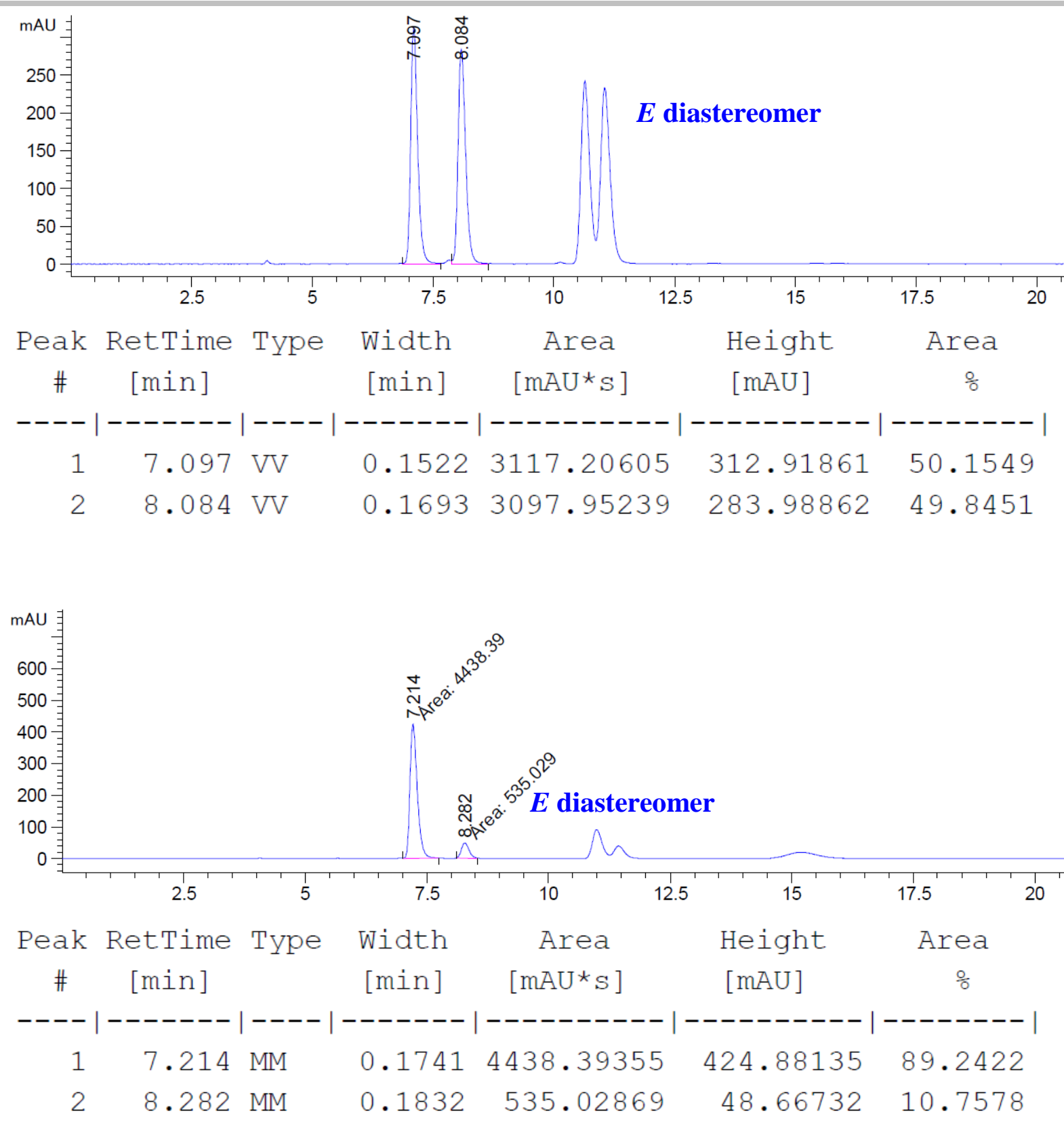


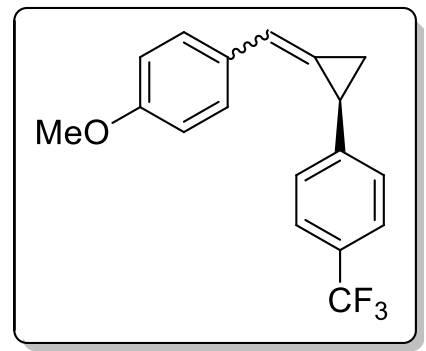

Prepared according to $\underline{\mathbf{G P 3}}$.

Yield: $36 \%$

$\mathbf{R}_{f}$ (20\% DCM in pentane): 0.23

Appearance: colourless oil

Z/E ratio: $2.7: 1$

${ }^{1} \mathbf{H}$ NMR (400 MHz, Chloroform- $\left.\boldsymbol{d}\right): \delta 7.57-7.23(\mathrm{~m}, 4 \mathrm{H} \boldsymbol{E}-\mathbf{6 a c}+6 \mathrm{H} \mathrm{Z}-6 \mathbf{6 a c}), 7.21(\mathrm{~d}, J=8.1 \mathrm{~Hz}, 2 \mathrm{H}$, $\boldsymbol{E}$-6ac), $6.95-6.89(\mathrm{~m}, 2 \mathrm{H} \boldsymbol{E}$-6ac $+1 \mathrm{H} \mathrm{Z-6ac}), 6.86(\mathrm{q}, J=2.3 \mathrm{~Hz}, 1 \mathrm{H}, \boldsymbol{E}-\mathbf{6 a c}), 6.78(\mathrm{~d}, J=8.8 \mathrm{~Hz}, 2 \mathrm{H}$, Z-6ac), 3.84 (s, 3H, E-6ac), 3.76 (s, 3H, Z-6ac), 2.92 (ddd, $J=8.8,4.4,2.0 \mathrm{~Hz}, 1 \mathrm{H}, \boldsymbol{Z}$-6ac), 2.72 (ddd, $J$ $=8.7,4.6,1.6 \mathrm{~Hz}, 1 \mathrm{H}, \boldsymbol{Z}-\mathbf{6 a c}), 2.15(\mathrm{td}, J=9.0,2.4 \mathrm{~Hz}, 1 \mathrm{H}, \boldsymbol{E}-\mathbf{- 6 a c}), 1.90(\mathrm{td}, J=8.9,2.0 \mathrm{~Hz}, 1 \mathrm{H}, \boldsymbol{Z}-\mathbf{6 a c})$, 1.55 (ddd, $1 \mathrm{H}, \boldsymbol{E}$-6ac), 1.27 (ddd, $J=9.0,4.4,2.2 \mathrm{~Hz}, 1 \mathrm{H}, \boldsymbol{Z}$-6ac).

${ }^{13}$ C NMR (101 MHz, Chloroform-d): $\delta 159.23$ (E-6ac), 159.07 (Z-6ac), 146.93 (E-6ac), 145.90 (Z6ac), 130.25 (E-6ac), 129.90 (Z-6ac), 128.27 (q, $J=32.3 \mathrm{~Hz}, \boldsymbol{Z}$-6ac), 128.26 (Z-6ac), 126.76, 126.71, 126.37, 125.66 (q, $J=3.8 \mathrm{~Hz}, \boldsymbol{Z}-\mathbf{6 a c}), 125.41$ (q, $J=3.8 \mathrm{~Hz}, \boldsymbol{E}-\mathbf{6 a c}), 125.32,124.47$ (q, $J=271.7 \mathrm{~Hz}, \boldsymbol{Z}$ 6ac), 123.92, 120.04 (Z-6ac), 119.49 (E-6ac), 114.21 (E-6ac), 114.16 (Z-6ac), 55.49 (E-6ac), 55.38 (Z6ac), 21.22 (Z-6ac), 17.97 (E-6ac), 16.63 (E-6ac), 14.51 (Z-6ac).

${ }^{19}$ F NMR (376 MHz, Chloroform-d): $\delta$-62.29.

IR (ATR): $v\left(\mathrm{~cm}^{-1}\right)$ 2962, 2934, 2838, 1607, 1510, 1320, 1246, 1108, 1067, 837, 524.

HRMS (APPI/LTQ-Orbitrap): calcd for $\mathrm{C}_{18} \mathrm{H}_{15} \mathrm{~F}_{3} \mathrm{O}^{+}[\mathrm{M}]^{+}$: 304.1070; found: 304.1076 .

$[\boldsymbol{\alpha}] \mathbf{D}^{20}=-123\left(c=0.5, \mathrm{CHCl}_{3}\right)$.

e.r. $(Z): 85: 15$ (Chiralpak IC, Hexane/IPA $=99.5 / 0.5, \lambda=230 \mathrm{~nm}, 1.00 \mathrm{~mL} / \mathrm{min}, \mathrm{t}_{\mathrm{R}}$ major $=4.98 \mathrm{~min}, \mathrm{t}_{\mathrm{R}}$ $\operatorname{minor}=5.66 \mathrm{~min})$ 

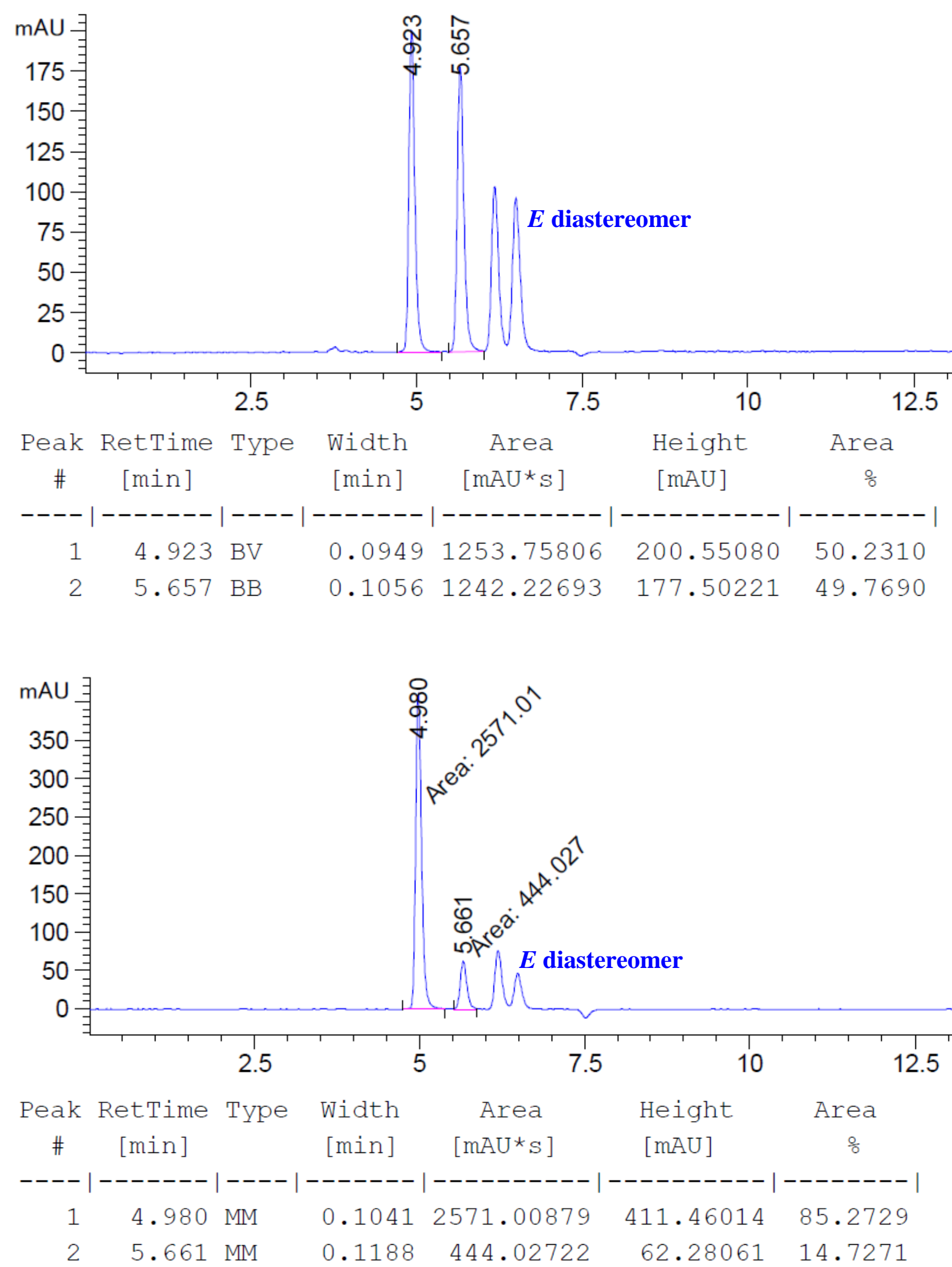


\section{(R)-1-(2-(4-Methoxybenzylidene)cyclopropyl)-3-methylbenzene 6ah}

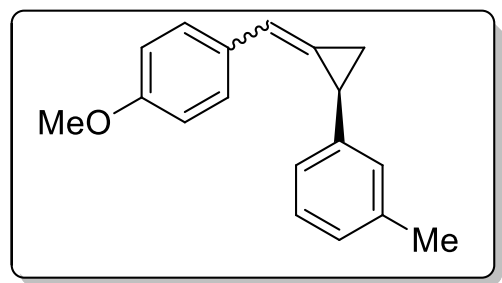

Prepared according to $\underline{\mathbf{G P 3}}$.

Yield: $93 \%$

$\mathbf{R}_{\boldsymbol{f}}(20 \%$ DCM in pentane): 0.23

Appearance: colourless oil

Z/E ratio: $2.7: 1$

${ }^{1}$ H NMR (400 MHz, Chloroform-d): $\delta 7.56$ (d, $\left.J=8.7 \mathrm{~Hz}, 2 \mathrm{H}, \boldsymbol{E}-\mathbf{6 a h}\right), 7.33$ (d, $J=8.7 \mathrm{~Hz}, 2 \mathrm{H}, Z$-6ah), 7.20 - 7.12 (m, 1H E-6ah + 1H Z-6ah), 7.04 - 6.90 (m, 5H $\boldsymbol{E - 6 a h ~ + ~ 3 H ~ Z - 6 a h ) , ~} 6.89$ (q, 1H, Z-6ah), 6.86 (q, 1H, E-6ah), 6.80 (d, J=8.8 Hz, 2H, Z-6ah), 3.85 (s, 3H, E-6ah), 3.77 (s, 3H, Z-6ah), 2.85 (ddd, $J=9.0,4.6,2.0 \mathrm{~Hz}, 1 \mathrm{H}, \boldsymbol{Z}-\mathbf{6 a h}), 2.67$ (ddd, $J=8.9,4.7,1.7 \mathrm{~Hz}, 1 \mathrm{H}, \boldsymbol{E}$-6ah), 2.33 (s, 3H, E-6ah), 2.32 (s, 3H, Z-6ah), 2.06 (td, $J=9.0,2.4 \mathrm{~Hz}, 1 \mathrm{H}, \boldsymbol{E}$-6ah), 1.82 (td, $J=8.9,2.0 \mathrm{~Hz}, 1 \mathrm{H}, \boldsymbol{Z}-\mathbf{6 a h}), 1.51$ (ddd, $J=$ 9.1, 4.7, 2.4 Hz, 1H, E-6ah), 1.24 (ddd, $J=8.8,4.6,2.0 \mathrm{~Hz}, 1 \mathrm{H}, \boldsymbol{Z}$-6ah).

${ }^{13}$ C NMR (101 MHz, Chloroform-d): $\delta 158.98$ (E-6ah), 158.79 (Z-6ah), 142.41 (E-6ah), 141.50 (Z6ah), 138.23 (Z-6ah), 138.13 (E-6ah), 130.76 (E-6ah), 130.41 (Z-6ah), 128.59, 128.40, 128.29, 128.13, 127.24, 127.15, 126.86, 126.81, 126.37, 125.19, 123.69 (E-6ah), 123.47 (Z-6ah), 119.51 (Z-6ah), 118.78 (E-6ah), 114.13 (E-6ah), 114.05 (Z-6ah), 55.46 (E-6ah), 55.34 (Z-6ah), 21.59 (Z-6ah), 21.53 (E6ah6ah), 21.44 (Z-6ah), 18.01 (E-6ah), 15.92 (E-6ah), 13.94 (Z-6ah).

IR (ATR): $v\left(\mathrm{~cm}^{-1}\right)$ 2961, 2931, 2835, 1606, 1509, 1245, 1172, 1033, 849, 698.

HRMS (APPI/LTQ-Orbitrap): calcd for $\mathrm{C}_{18} \mathrm{H}_{18} \mathrm{O}^{+}[\mathrm{M}]^{+}:$250.1352; found: 250.1350 .

$[\boldsymbol{\alpha}] \mathbf{D}^{\mathbf{2 0}}=-363\left(c=1.0, \mathrm{CHCl}_{3}\right)$.

e.r. $(Z)$ : $92: 8$ (Chiralpak IG, Hexane $/ \mathrm{IPA}=99.5 / 0.5, \lambda=254 \mathrm{~nm}, 1.00 \mathrm{~mL} / \mathrm{min}, \mathrm{t}_{\mathrm{R}}$ major $=7.39 \mathrm{~min}, \mathrm{t}_{\mathrm{R}}$ minor $=8.22 \mathrm{~min})$ 

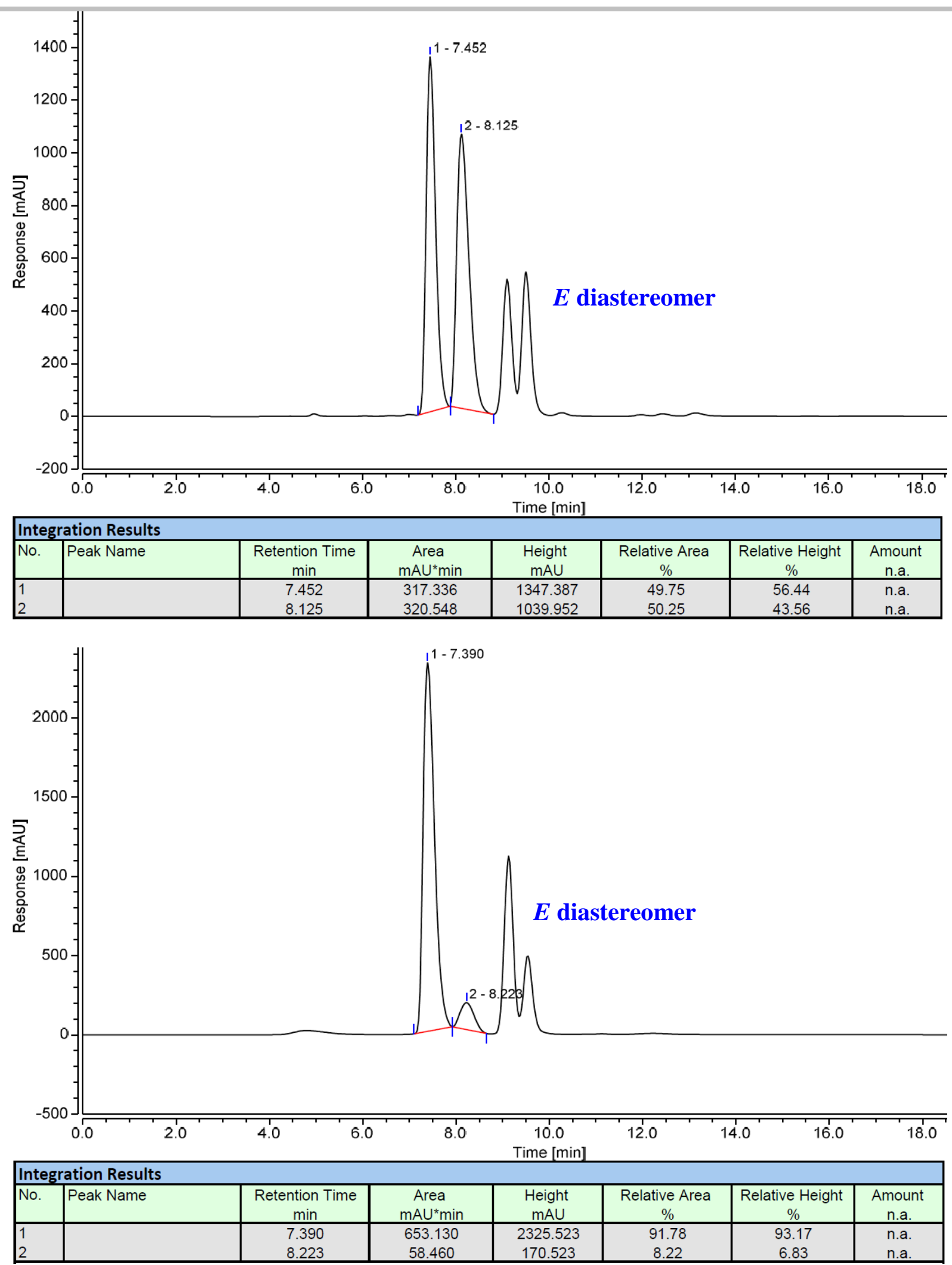


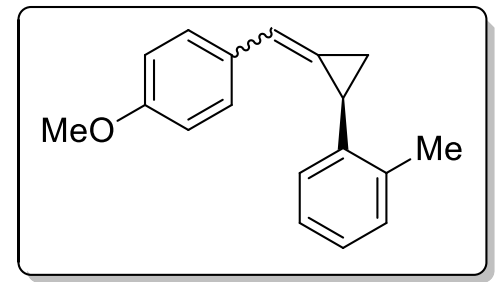

Prepared according to $\underline{\mathbf{G P 3}}$.

Yield: $46 \%$

$\mathbf{R}_{f}(20 \%$ DCM in pentane): 0.23

Appearance: colourless oil

Z/E ratio: $1: 1.3$

${ }^{1}$ H NMR (400 MHz, Chloroform-d): $\delta 7.69$ (d, $J=8.3 \mathrm{~Hz}, 2 \mathrm{H}, \boldsymbol{E}$-6ai), 7.55 (d, $J=8.3 \mathrm{~Hz}, 2 \mathrm{H}, \boldsymbol{Z}$-6ai), $7.44-7.23$ (m, 3H E-6ai + 3H Z-6ai), 7.16 (t, $J=7.6$ Hz, 1H, Z-6ai), 7.13 - 7.04 (m, 4H, E-6ai), 6.99 (d, $J=8.4 \mathrm{~Hz}, 2 \mathrm{H}, \boldsymbol{Z}$-6ai), 6.94 (d, $J=7.6 \mathrm{~Hz}, 1 \mathrm{H}, \boldsymbol{Z}$-6ai), 3.99 (s, 3H, E-6ai), 3.95 (s, 3H, Z-6ai), 3.13 - 3.05 (m, 1H, Z-6ai), 2.92 - 2.83 (m, 1H, E-6ai), 2.69 (s, 3H, Z-6ai), 2.67 (s, 3H, E-6ai), 2.21 (td, $J=$ 8.8, 2.2 Hz, 1H, E-6ai), 1.98 (t, J = 8.7 Hz, 1H, Z-6ai), $1.62-1.54$ (m, 1H, E-6ai), 1.26 - 1.18 (m, 1H, Z-6ai).

${ }^{13}$ C NMR (101 MHz, Chloroform-d): $\delta 158.94$ (E-6ai), 158.86 (Z-6ai), 139.55 (E-6ai), 139.22 (Z-6ai), 137.54 (E-6ai), 137.23 (Z-6ai), 130.89 (E-6ai), 130.54 (Z-6ai), 129.90 (Z-6ai), 129.83 (E-6ai), 128.37 (Z-6ai), 128.04 (E-6ai), 126.37, 126.29, 126.24, 126.01, 125.86, 125.80, 125.62, 124.44, 120.41, 119.26, 114.12, 55.46 (E-6ai), 55.38 (Z-6ai), 19.79 (E-6ai), 19.75 (Z-6ai), 19.02 (Z-6ai), 15.76 (E-6ai), 14.28 (E-6ai), 12.94 (Z-6ai).

IR (ATR): $v\left(\mathrm{~cm}^{-1}\right)$ 2959, 2931, 2835, 1605, 1509, 1489, 1461, 1244, 1172, 1033, 851, 752.

HRMS (APPI/LTQ-Orbitrap): calcd for $\mathrm{C}_{18} \mathrm{H}_{18} \mathrm{O}^{+}[\mathrm{M}]^{+}:$250.1352; found: 250.1352 .

$[\boldsymbol{\alpha}] \mathbf{D}^{\mathbf{2 0}}=-196\left(c=1.0, \mathrm{CHCl}_{3}\right)$.

e.r. $(Z)$ : $89: 11$ (Chiralpak IG, Hexane/IPA $=99.5 / 0.5, \lambda=254 \mathrm{~nm}, 1.00 \mathrm{~mL} / \mathrm{min}, \mathrm{t}_{\mathrm{R}}$ major $=7.34 \mathrm{~min}, \mathrm{t}_{\mathrm{R}}$ $\operatorname{minor}=8.37 \mathrm{~min}$ )

e.r. $(\boldsymbol{E})$ : $61: 39$ (Chiralpak IG, Hexane/IPA $=99.5 / 0.5, \lambda=254 \mathrm{~nm}, 1.00 \mathrm{~mL} / \mathrm{min}, \mathrm{t}_{\mathrm{R}}$ major $=10.3 \mathrm{~min}, \mathrm{t}_{\mathrm{R}}$ minor $=11.4 \mathrm{~min})$ 

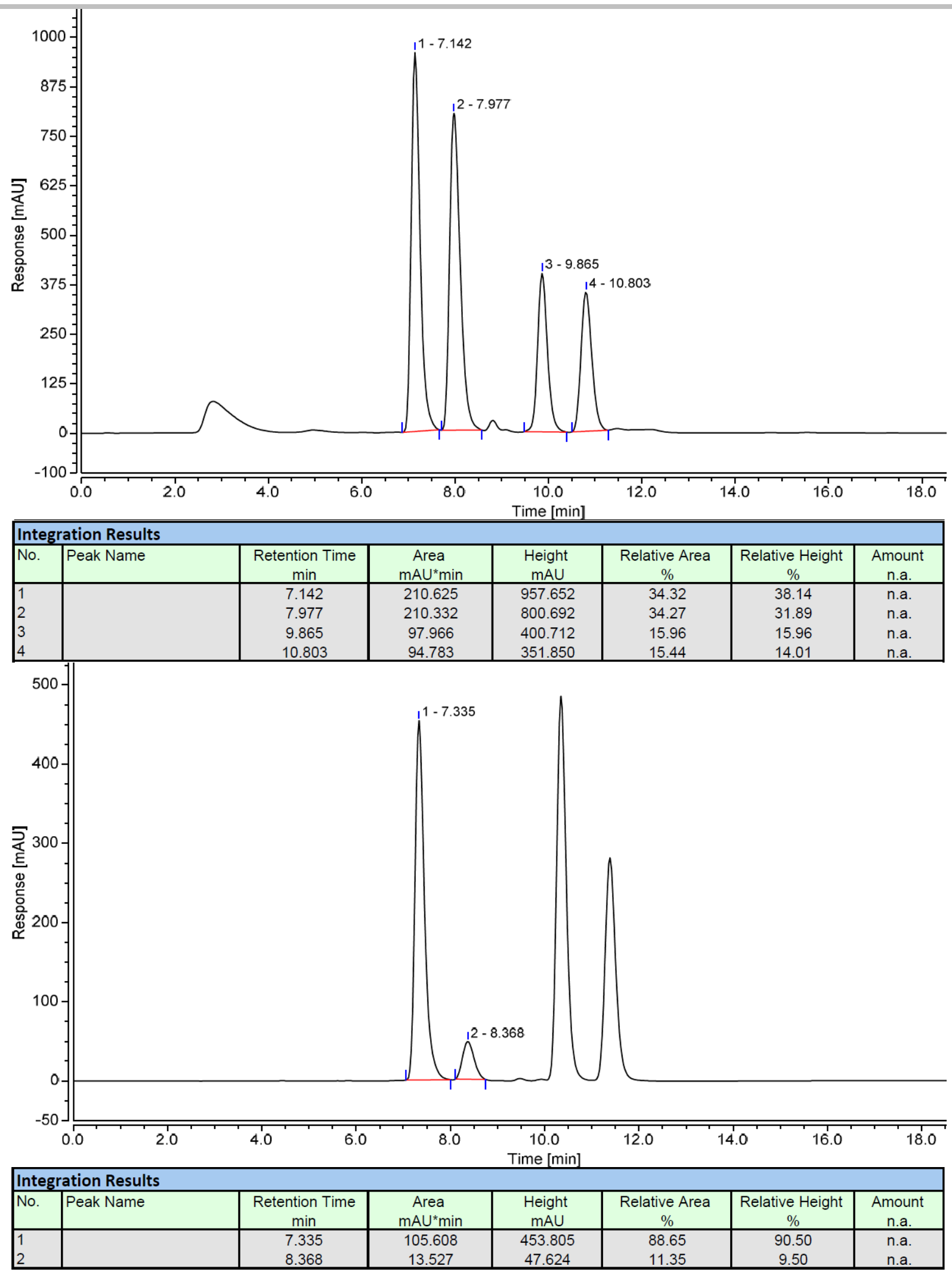


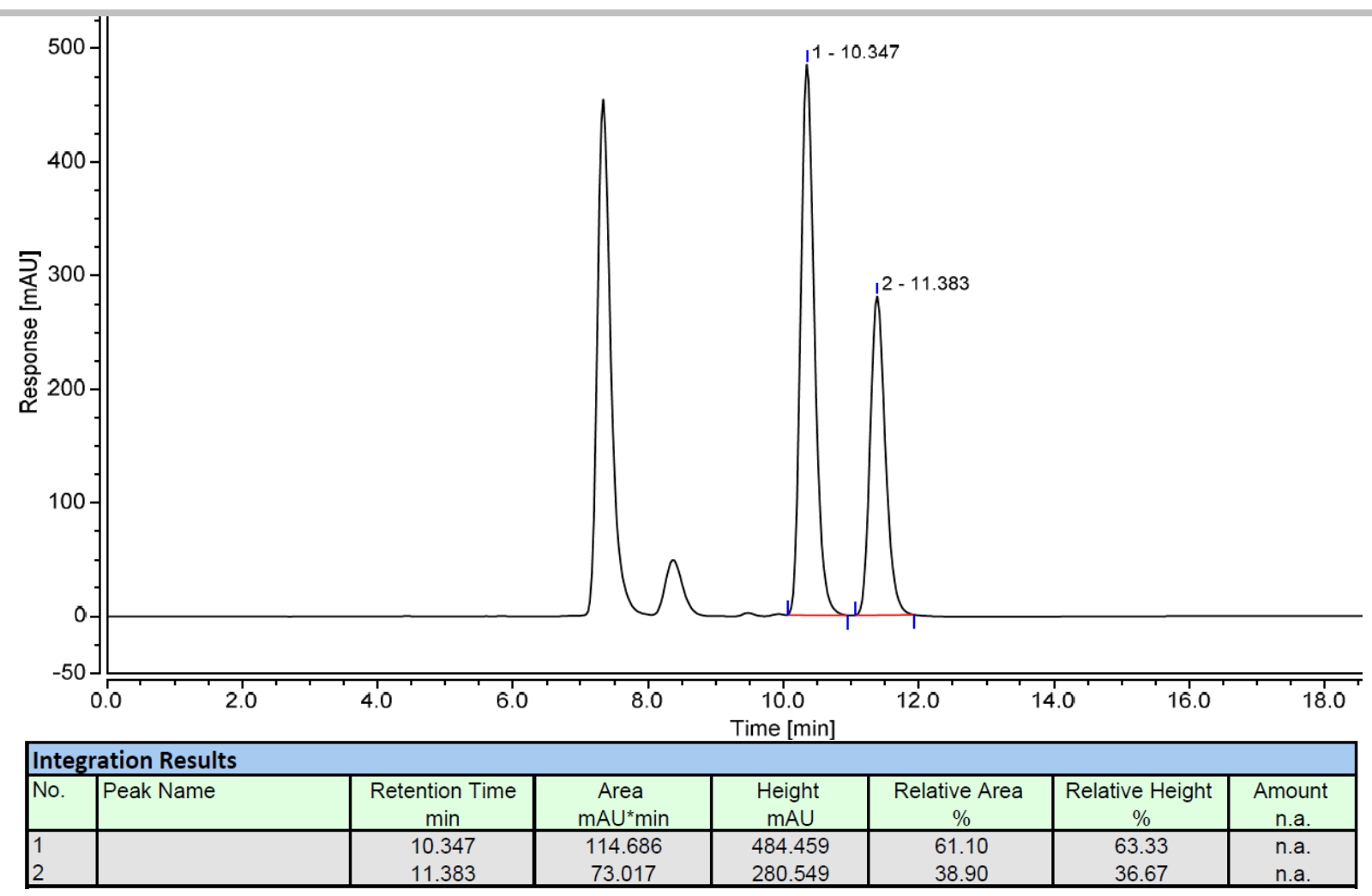




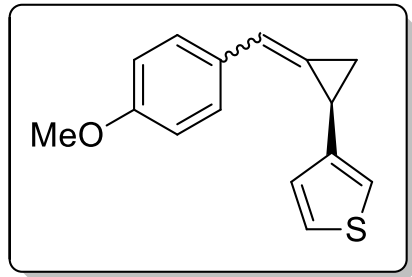

Prepared according to $\underline{\mathbf{G P 3}}$ with the following modification: reaction run at $25^{\circ} \mathrm{C}$.

Yield: $46 \%$

$\mathbf{R}_{f}(20 \%$ DCM in pentane): 0.23

Appearance: colourless oil

Z/E ratio: $2.3: 1$

${ }^{1}$ H NMR (400 MHz, Chloroform-d): $\delta 7.52$ (d, $J=8.7$ Hz, 2H, E-6aj), 7.34 (d, $J=8.7$ Hz, 2H, Z-6aj), $7.24-7.19$ (m, 3H, E-6aj), 7.00 (dd, $J=3.2,1.2 \mathrm{~Hz}, 1 \mathrm{H}, \boldsymbol{E}$-6aj), 6.94 (dd, $J=3.1,1.3 \mathrm{~Hz}, 1 \mathrm{H}, \boldsymbol{Z}$-6aj), $6.92-6.77$ (m, 2H E-6aj + 5H Z-6aj), 3.83 (s, 3H, E-6aj), 3.78 (s, 3H, Z-6aj), 2.94 (ddd, J = 8.9, 4.6, $2.1 \mathrm{~Hz}, 1 \mathrm{H}, \boldsymbol{Z}$-6aj), 2.72 (ddd, $J=8.8,4.8,1.7 \mathrm{~Hz}, 1 \mathrm{H}, \boldsymbol{E}$-6aj), 2.02 (td, $J=8.9,2.4 \mathrm{~Hz}, 1 \mathrm{H}, \boldsymbol{E}$-6aj), 1.78 (td, $J=8.8,1.9 \mathrm{~Hz}, 1 \mathrm{H}, \boldsymbol{Z}$-6aj), 1.50 (ddd, $J=9.1,4.7,2.4 \mathrm{~Hz}, 1 \mathrm{H}, \boldsymbol{E}$-6aj), 1.24 (ddd, 1H, Z-6aj).

${ }^{13}$ C NMR (101 MHz, Chloroform-d): $\delta 159.01$ (E-6aj), 158.85 (Z-6aj), 142.92 (E-6aj), 141.91 (Z-6aj), 130.71 (E-6aj), 130.35 (Z-6aj), 128.34 (Z-6aj), 128.11 (E-6aj), 126.61, 126.54, 125.98, 125.80, 125.62, 125.11, 119.73 (Z-6aj), 119.49 (E-6aj), 119.34 (Z-6aj), 118.66 (E-6aj), 114.13 (E-6aj), 114.08 (Z-6aj), 55.46 (E-6aj), 55.38 (Z-6aj), 17.13 (Z-6aj), 15.22 (E-6aj), 13.79 (E-6aj), 12.93 (Z-6aj).

IR (ATR): $v\left(\mathrm{~cm}^{-1}\right)$ 3101, 3034, 2962, 2931, 2834, 1606, 1510, 1247, 1173, 1033, 851.

HRMS (APPI/LTQ-Orbitrap): calcd for $\mathrm{C}_{15} \mathrm{H}_{14} \mathrm{OS}^{+}[\mathrm{M}]^{+}: 242.0760$; found: 242.0754 .

$[\boldsymbol{\alpha}]_{\mathbf{D}}^{\mathbf{2 0}}=-221\left(c=1.0, \mathrm{CHCl}_{3}\right)$.

e.r. $(Z)$ : $91: 9$ (Chiralpak IG, Hexane/IPA $=99.5 / 0.5, \lambda=230 \mathrm{~nm}, 1.00 \mathrm{~mL} / \mathrm{min}, \mathrm{t}_{\mathrm{R}}$ major $=8.91 \mathrm{~min}, \mathrm{t}_{\mathrm{R}}$ minor $=9.95 \mathrm{~min})$ 


\section{SUPPORTING INFORMATION}

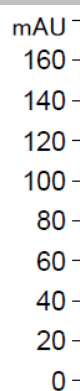

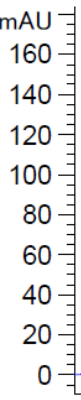

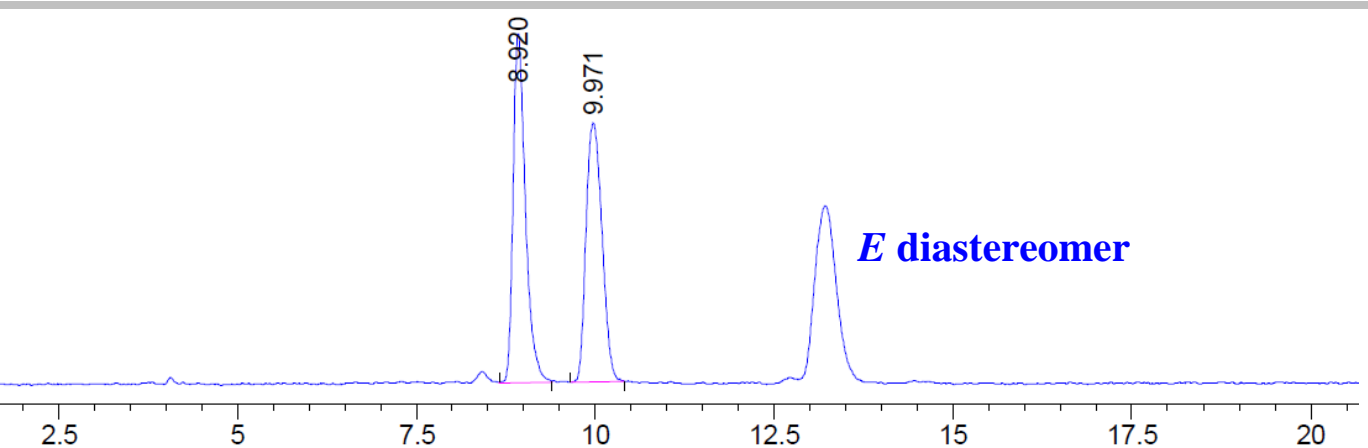

Peak RetTime Type

\begin{tabular}{|c|c|c|c|c|c|}
\hline$\#$ & {$[\min ]$} & [min] & {$\left[\mathrm{mAU}^{*} \mathrm{~s}\right]$} & [mAU] & 응 \\
\hline- & $----\mid--$ & ------ & ---------- & -------- & -------- \\
\hline 1 & $8.920 \mathrm{VV}$ & 0.1839 & 2088.97339 & 174.25636 & 51.0820 \\
\hline 2 & $9.971 \mathrm{BB}$ & 0.2506 & 2000.47681 & 129.21857 & 48.9180 \\
\hline
\end{tabular}

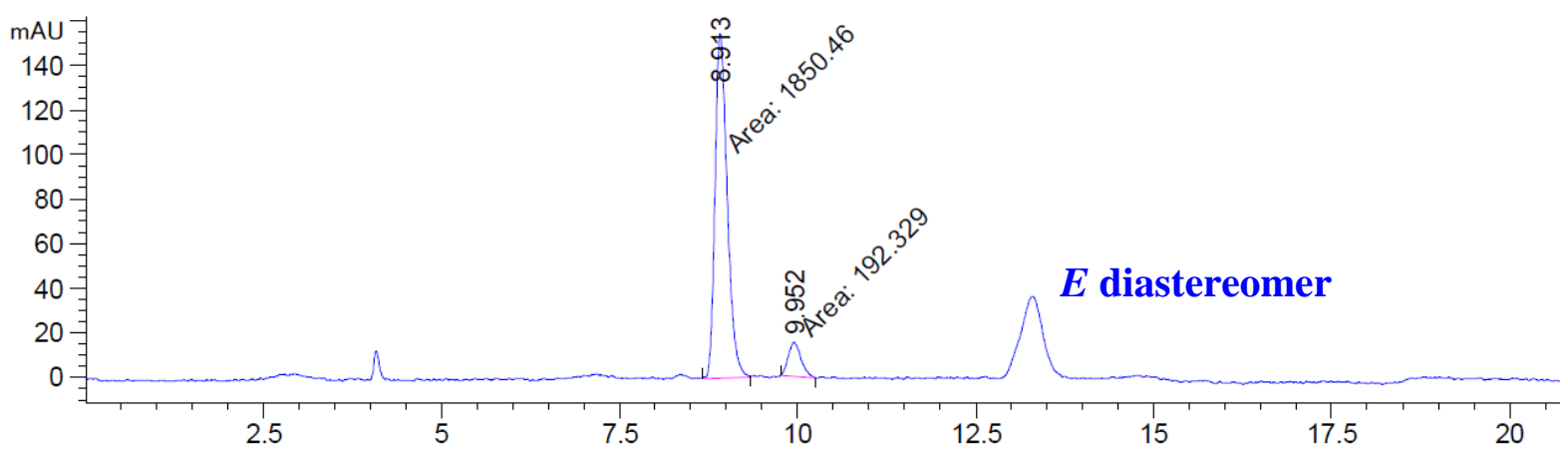

Peak RetTime Type Width Area Height Area

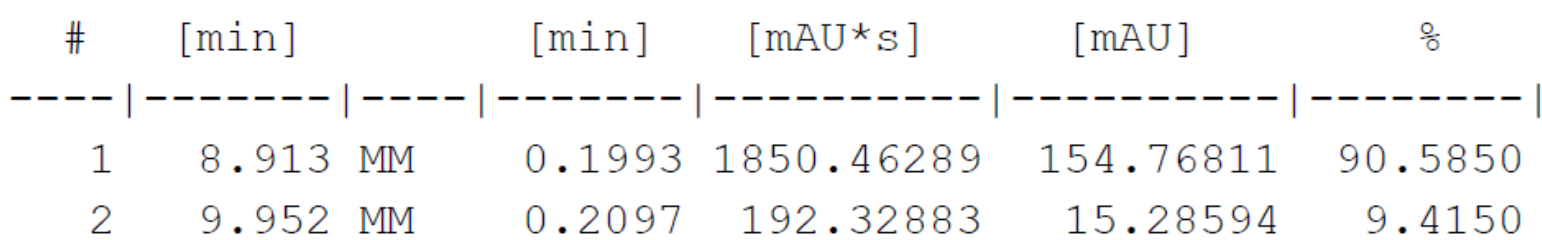




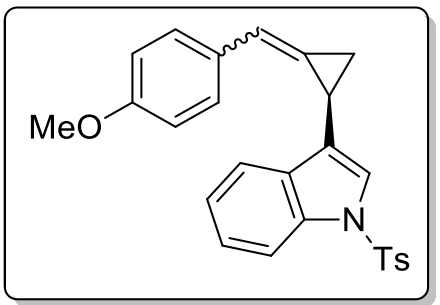

Prepared according to $\underline{\mathbf{G P 3}}$ with the following modification: reaction run at $25^{\circ} \mathrm{C}$.

Yield: $86 \%$

$\mathbf{R}_{f}(50 \%$ DCM in pentane): 0.25

Appearance: yellow solid

Z/E ratio: $6.5: 1$

${ }^{1}$ H NMR (400 MHz, Chloroform-d): $\delta 7.99$ (m, 1H $\boldsymbol{E}$-6ak + 1H Z-6ak), 7.74 (d, $J=8.3 \mathrm{~Hz}, 1 \mathrm{H}, \boldsymbol{E}$ 6ak), 7.64 (d, $J=8.1 \mathrm{~Hz}, 1 \mathrm{H}, Z$-6ak), 7.59 (d, $J=8.4 \mathrm{~Hz}, 2 \mathrm{H}, \boldsymbol{Z}$-6ak), 7.54 (d, $J=8.7 \mathrm{~Hz}, 1 \mathrm{H}, \boldsymbol{E}$-6ak), $7.39-7.18$ (m, 10H E-6ak + 4H Z-6ak), 7.15 (d, $J=8.1 \mathrm{~Hz}, 2 \mathrm{H}, \boldsymbol{Z}$-6ak), 7.06 (s, 1H, Z-6ak), 6.97 6.90 (m, 1H $\boldsymbol{E}$-6ak + 1H Z-6ak), 6.77 (d, $J=8.7$ Hz, 2H, Z-6ak), 3.84 (s, 3H, E-6ak), 3.82 (s, 3H, Z6ak), 2.90 (ddd, $J=8.4,4.5,2.1 \mathrm{~Hz}, 1 \mathrm{H}, \boldsymbol{Z}$-6ak), 2.68 (ddd, 1H, E-6ak), 2.34 (s, 3H, Z-6ak), 2.33 (s, 3H, E-6ak6ak), $2.05(\mathrm{td}, J=8.8,2.4 \mathrm{~Hz}, 1 \mathrm{H}, \boldsymbol{E}$-6ak), $1.86(\mathrm{td}, J=8.6,1.9 \mathrm{~Hz}, 1 \mathrm{H}, \boldsymbol{Z}$-6ak), $1.59-1.50$ (m, 1H, E-6ak), $1.31-1.24$ (m, 1H, Z-6ak).

${ }^{13}$ C NMR (101 MHz, Chloroform-d): $\delta 159.13$ (E-6ak), 158.91 (Z-6ak), 144.87 (E-6ak), 144.78 (Z6ak), 135.60 (Z-6ak), 135.49 (E-6ak), 135.47 (E-6ak), 135.20 (Z-6ak), 130.88 (Z-6ak), 130.49 (E-6ak), 130.24 (Z-6ak), 129.96 (E-6ak), 129.87 (Z-6ak), 128.46 (Z-6ak), 128.16 (E-6ak), 126.93 (E-6ak), 126.90 (Z-6ak), 124.90 (Z-6ak), 124.85 (E-6ak), 123.67 (E-6ak), 123.36 (E-6ak), 123.31 (Z-6ak), 123.20 (E-6ak), 123.00 (Z-6ak), 122.74 (Z-6ak), 122.56 (Z-6ak), 120.46 (Z-6ak), 119.88 (E-6ak), 119.69 (Z-6ak), 119.55 (E-6ak, 114.18 (E-6ak), 114.10 (Z-6ak), 114.03 (Z-6ak), 113.87 (E-6ak), 55.47 (E-6ak), 55.38 (Z-6ak), 21.68 (Z-6ak), 13.94 (E-6ak), 12.89 (Z-6ak), 12.14 (Z-6ak), 9.02 (E-6ak).

IR (ATR): $v\left(\mathrm{~cm}^{-1}\right)$ 3050, 2965, 2836, 1607, 1368, 1247, 1173, 1123, 746, 667, 573.

HRMS (ESI/QTOF): calcd for $\mathrm{C}_{26} \mathrm{H}_{24} \mathrm{NO}_{3} \mathrm{~S}^{+}[\mathrm{M}+\mathrm{H}]^{+}$: 430.1471 ; found: 430.1469 .

$[\boldsymbol{\alpha}] \mathbf{D}^{\mathbf{2 0}}=-229\left(c=1.0, \mathrm{CHCl}_{3}\right)$.

e.r. $(Z): 91: 9$ (Chiralpak IA, Hexane/IPA $=95: 5, \lambda=254 \mathrm{~nm}, 1.00 \mathrm{~mL} / \mathrm{min}, \mathrm{t}_{\mathrm{R}}$ major $=9.81 \mathrm{~min}$, $\mathrm{t}_{\mathrm{R}}$ minor $=11.2 \mathrm{~min}$ )

Melting point: $110-112^{\circ} \mathrm{C}$ 

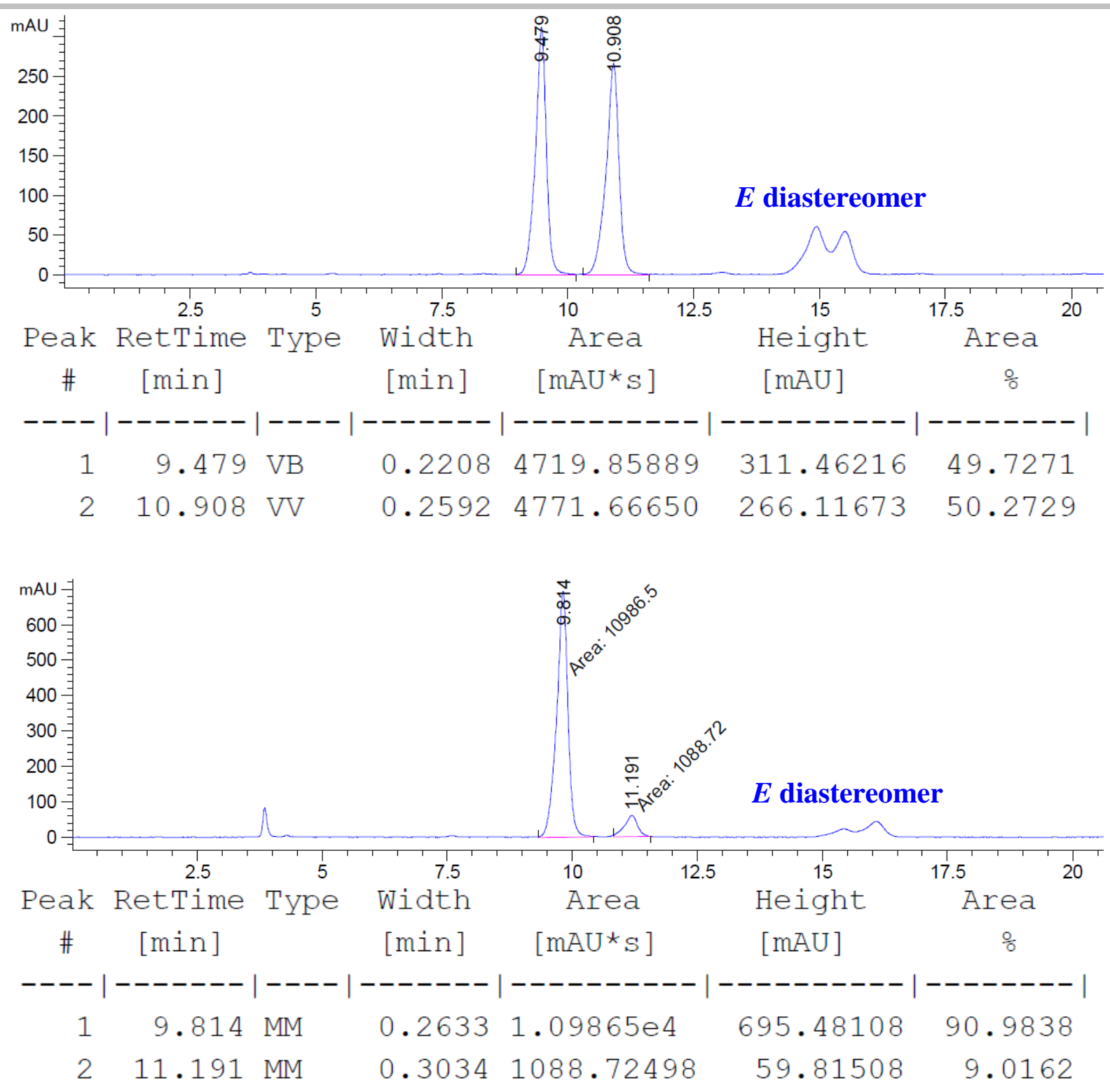


\section{Recrystallization of (R)-3-(2-(4-methoxybenzylidene)cyclopropyl)-1-tosyl-1H-indole 6ak:}

(R)-3-(2-(4-methoxybenzylidene)cyclopropyl)-1-tosyl-1H-indole could be enantioenriched by recrystallization (85\% yield) from Chloroform/Hexane (1:100).

Yield of recrystallization: $85 \%$

$Z / E$ ratio: $6.8: 1$

e.r. (Z): $98: 2$ (Chiralpak IA, Hexane/IPA $=95: 5, \lambda=254 \mathrm{~nm}, 1.00 \mathrm{~mL} / \mathrm{min}, \mathrm{t}_{\mathrm{R}}$ major $=9.96 \mathrm{~min}, \mathrm{t}_{\mathrm{R}}$ minor $=11.7 \mathrm{~min})$

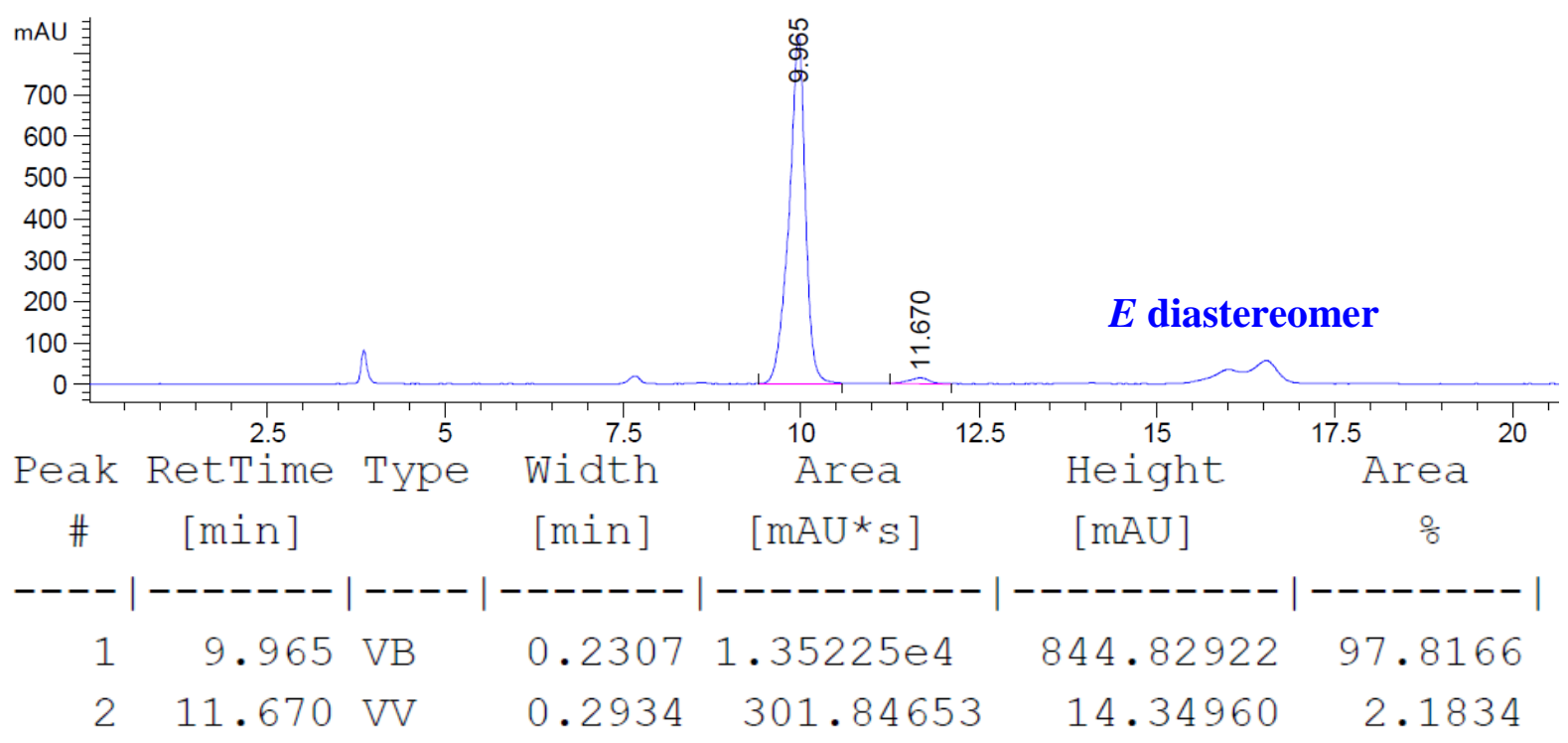




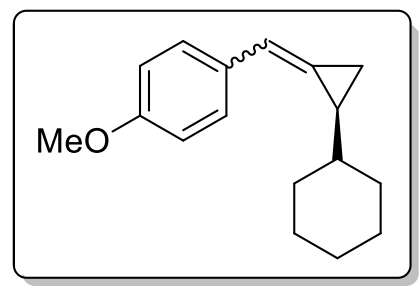

Prepared according to $\underline{\mathbf{G P 3}}$ with the following modification: reaction run at $25^{\circ} \mathrm{C}$.

Yield: $66 \%$

$\mathbf{R}_{f}(20 \%$ DCM in pentane): 0.29

Appearance: colourless oil

Z/E ratio: 3.1:1

${ }^{1}$ H NMR (400 MHz, Chloroform-d): $\delta 7.47$ (d, $\left.J=8.7 \mathrm{~Hz}, 2 \mathrm{H}, \boldsymbol{Z}-6 \mathbf{a l}\right), 7.40$ (d, $\left.J=8.7 \mathrm{~Hz}, 2 \mathrm{H}, \boldsymbol{E}-6 \mathbf{a l}\right)$, $6.93-6.81(\mathrm{~m}, 2 \mathrm{H} \boldsymbol{E}-\mathbf{6 a l}+2 \mathrm{H} \mathrm{Z}$-6al), $6.71(\mathrm{q}, J=2.1 \mathrm{~Hz}, 1 \mathrm{H}, \boldsymbol{Z}-\mathbf{6 a l}), 6.66$ (q, $J=2.0 \mathrm{~Hz}, 1 \mathrm{H}, \boldsymbol{E}$-6al), 3.82 (s, 3H, E-6al), 3.81 (s, 3H, Z-6al), 1.91 - 0.82 (m, 14H $\boldsymbol{E}$-6al + 14H Z-6al).

${ }^{13}$ C NMR (101 MHz, Chloroform- $\boldsymbol{d}$ ): $\delta 158.59$ (Z-6al), 158.52 (E-6al), 131.48 (Z-6al), 131.36 (E-6al), 128.04 (E-6al), 127.74 (Z-6al), 127.39 (Z-6al), 126.86 (E-6al), 118.45 (E-6al), 116.71 (Z-6al), 114.01 (Z-6al), 113.92 (E-6al), 55.43, 55.40, 41.92, 40.14, 33.60, 33.33, 32.78, 32.62, 30.40, 29.86, 26.59, 26.55, 26.52, 26.46, 26.40, 26.36, 23.29, 19.65, 9.37, 5.04.

IR (ATR): $v\left(\mathrm{~cm}^{-1}\right)$ 2920, 2849, 1607, 1509, 1447, 1303, 1244, 1171, 1036, 844.

HRMS (ESI/QTOF): calcd for $\mathrm{C}_{17} \mathrm{H}_{23} \mathrm{O}^{+}[\mathrm{M}+\mathrm{H}]^{+}:$: 243.1743; found: 243.1743 .

$[\boldsymbol{\alpha}]_{\mathbf{D}^{\mathbf{2 0}}}=-35.8\left(c=1.0, \mathrm{CHCl}_{3}\right)$.

e.r. $(Z)$ : $50: 50$ (Chiralpak IB, Hexane/IPA $=99.95 / 0.05, \lambda=254 \mathrm{~nm}, 0.70 \mathrm{~mL} / \mathrm{min}, \mathrm{t}_{\mathrm{R}}=8.19 \mathrm{~min}, 9.00$ $\min )$

e.r. $(\boldsymbol{E})$ : 86:14 (Chiralpak IB, Hexane/IPA $=99.95 / 0.05, \lambda=254 \mathrm{~nm}, 0.70 \mathrm{~mL} / \mathrm{min}, \mathrm{t}_{\mathrm{R}}$ major $=7.37 \mathrm{~min}$, $\left.\mathrm{t}_{\mathrm{R}} \operatorname{minor}=7.80 \mathrm{~min}\right)$ 


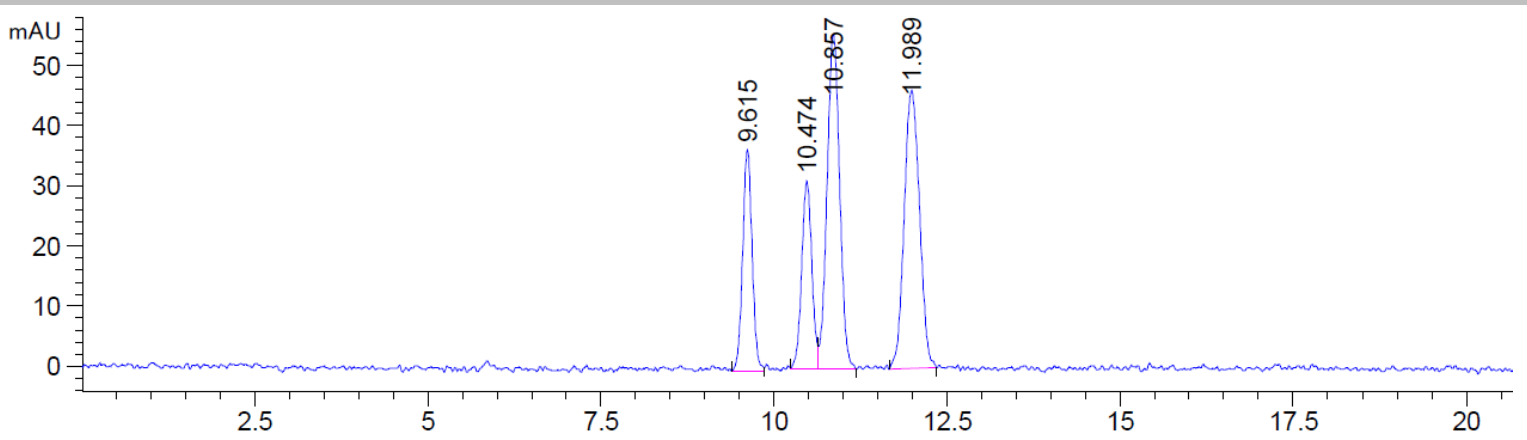

Peak RetTime Type Width Area Height Area

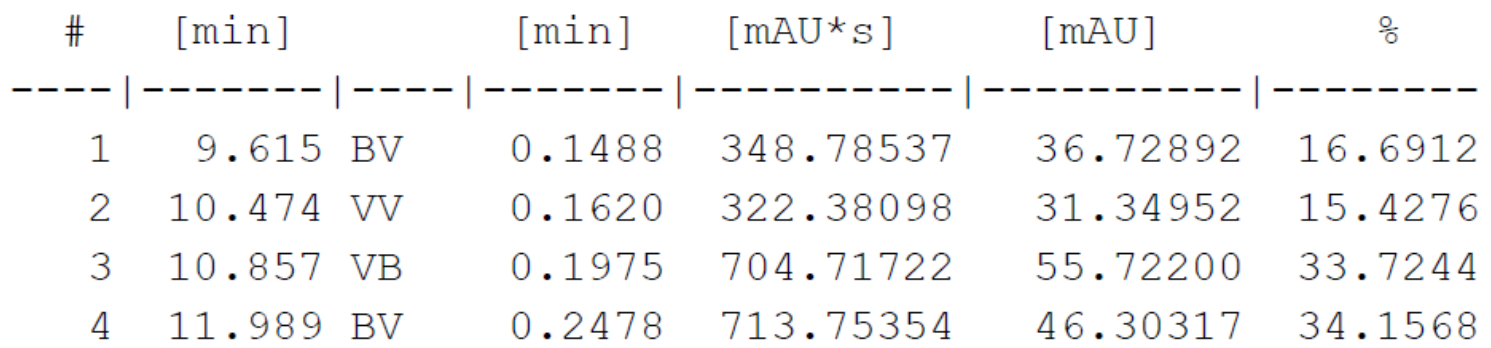

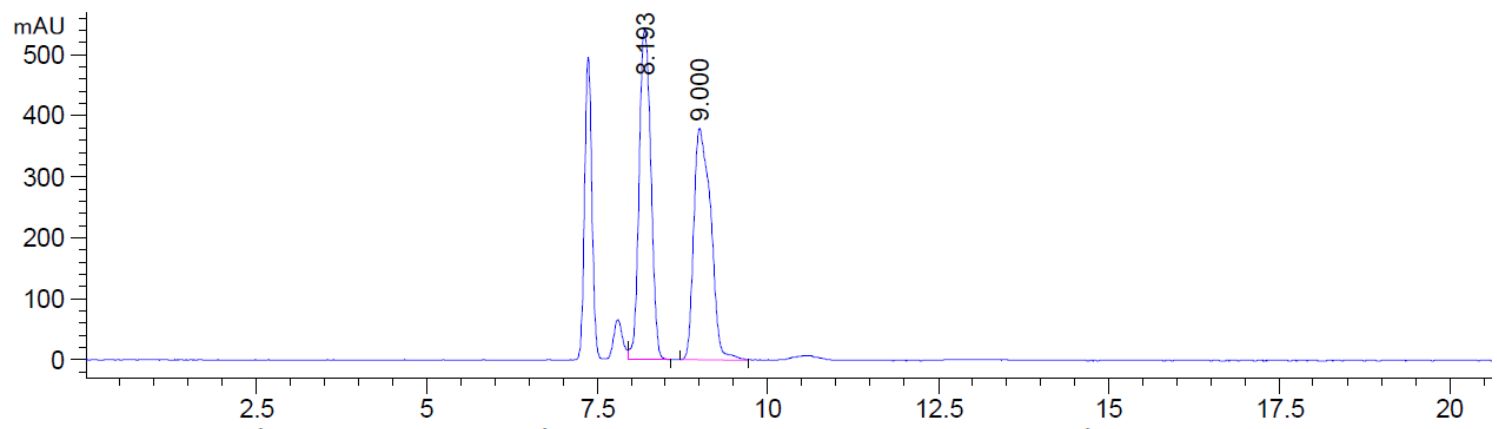

Peak RetTime Type Width Area Height Area
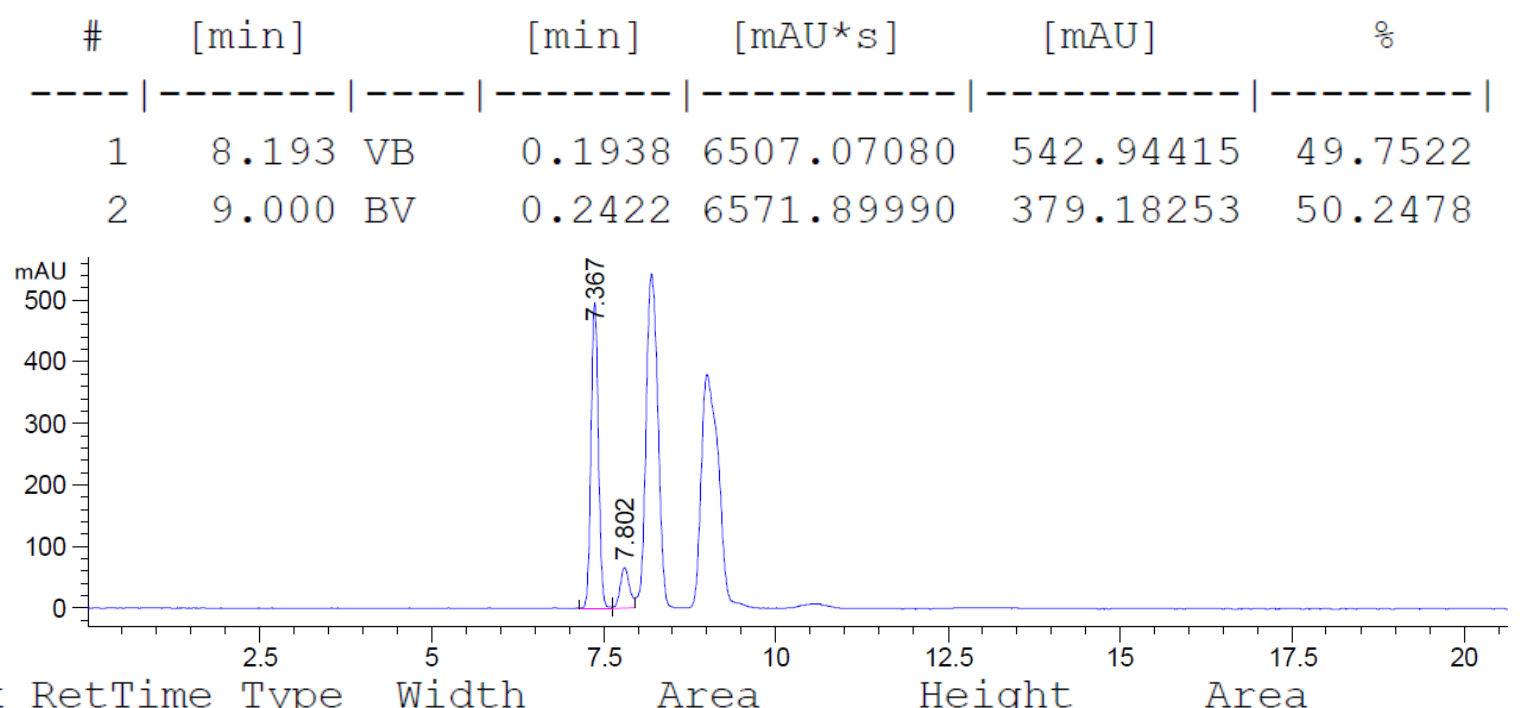

Peak RetTime Type Width Area Height Area

$\begin{array}{cccccc}\# & {[\mathrm{~min}]} & {[\mathrm{min}]} & {\left[\mathrm{mAU}{ }^{*} \mathrm{~S}\right]} & {[\mathrm{mAU}]} & \frac{\circ}{0} \\ -1 & 7.367 \mathrm{VV} & 0.1141 & 3676.87402 & 498.06317 & 85.6480 \\ 2 & 7.802 \mathrm{VV} & 0.1408 & 616.13397 & 66.07092 & 14.3520\end{array}$




\section{Derivatization of chiral alkylidenecyclopropanes}

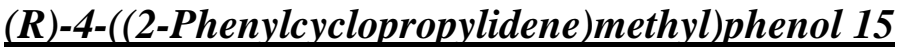

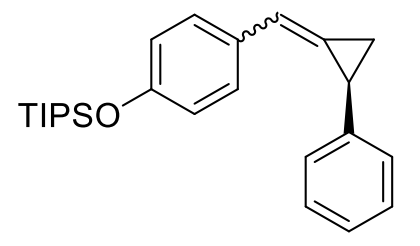

$$
\underset{\operatorname{THF}(0.30 \mathrm{M}), \mathrm{RT}, 5 \mathrm{~min}}{\stackrel{n-\mathrm{Bu}_{4} \mathrm{NF}(2.00 \text { equiv })}{\longrightarrow}}
$$

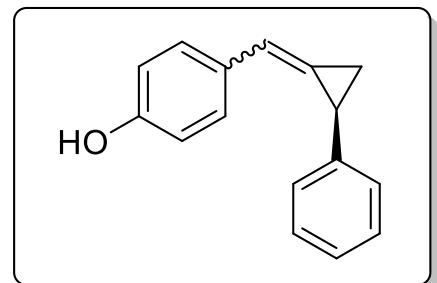

(R)-Triisopropyl(4-((2-phenylcyclopropylidene)methyl)phenoxy)silane 6ca (0.05 mmol, 1.00 equiv.) was dissolved in THF $(0.18 \mathrm{~mL}, 0.30 \mathrm{M})$ and tetra- $n$-butylammonium fluoride $(0.10 \mathrm{mmol}, 2.00$ equiv, 1.00 $\mathrm{M}$ in THF) was added dropwise. The solution was stirred at room temperature until TLC showed full conversion of starting material (approximately 5 minutes). The solution was quenched by addition of water (approximately $0.40 \mathrm{~mL}$ ) and diluted with EtOAc. The layers were separated and the aqueous layer was washed $(1 \times)$ with EtOAc. The combined organics were dried over $\mathrm{MgSO}_{4}$, filtered and concentrated under reduced pressure. Purification via flash column chromatogtraphy $\left(\mathrm{SiO}_{2}, 20 \%\right.$ EtOAc in pentane $)$ afforded the title compound 15.

\section{Yield: $81 \%$}

Appearance: white solid

$\mathrm{Z} / \boldsymbol{E}$ ratio: $3.2: 1$

${ }^{1}$ H NMR (400 MHz, Chloroform-d): $\delta 7.48(\mathrm{~d}, 1 \mathrm{H}, \boldsymbol{E}-\mathbf{1 3}), 7.31-7.22(\mathrm{~m}, 3 \mathrm{H} \boldsymbol{E}-\mathbf{1 3}+4 \mathrm{H} \mathrm{Z}-13), 7.21-$ 7.09 (m, 3H $\boldsymbol{E}-13$ + 3H Z-13), $6.89-6.81$ (m, 3H $\boldsymbol{E}-13$ + 1H Z-13), 6.69 (d, J = 8.6 Hz, 2H, Z-13), 4.72 (bs, 1H, E-13), 4.62 (bs, 1H, Z-13), 2.87 (ddd, $J=9.0,4.6,2.1 \mathrm{~Hz}, 1 \mathrm{H}, \boldsymbol{Z}-13$ ), 2.68 (ddd, $J=8.9,4.8,1.8$ $\mathrm{Hz}, 1 \mathrm{H}, \boldsymbol{E}-13), 2.06(\mathrm{td}, J=9.0,2.4 \mathrm{~Hz}, 1 \mathrm{H}, \boldsymbol{E}-\mathbf{1 3}), 1.82(\mathrm{td}, J=8.9,2.0 \mathrm{~Hz}, 1 \mathrm{H}, \boldsymbol{Z}-13), 1.49$ (ddd, $J=$ 9.2, 4.7, $2.5 \mathrm{~Hz}, 1 \mathrm{H}, \boldsymbol{E}-\mathbf{1 3}), 1.23$ (ddd, $J=8.8,4.7,2.0 \mathrm{~Hz}, 1 \mathrm{H}, Z-13$ ).

${ }^{13}$ C NMR (101 MHz, Chloroform-d): $\delta 154.90$ (E-13), 154.73 (Z-13), 142.45 (E-13), 141.46 (Z-13), 130.94 (E-13), 130.59 (Z-13), 128.96, 128.70, 128.51, 128.36, 126.59, 126.54, 126.32, 126.07, 126.03, 125.12, 119.52 (Z-13), $118.83(\boldsymbol{E}-13), 115.60$ (E-13), $115.53(\boldsymbol{Z}-13,21.48(\boldsymbol{Z}-13), 18.08(\boldsymbol{E}-13), 15.99$ (E-13), $13.97(Z-13)$.

IR (ATR): $v\left(\mathrm{~cm}^{-1}\right)$ 3352, 3061, 3027, 2942, 2891, 2865, 1607, 1511, 1452, 1228, 1169, 696, 517.

HRMS (APPI/LTQ-Orbitrap): calcd for $\mathrm{C}_{16} \mathrm{H}_{14} \mathrm{O}^{+}[\mathrm{M}]^{+}$: 222.1039; found: 222.1037.

$[\alpha]_{\mathbf{D}^{20}}=-220\left(c=1.0, \mathrm{CHCl}_{3}\right)$.

e.r. $(Z): 87: 13$ (Chiralpak IG, Hexane $/ \mathrm{IPA}=90 / 10, \lambda=254 \mathrm{~nm}, 1.00 \mathrm{~mL} / \mathrm{min}, \mathrm{t}_{\mathrm{R}}$ major $=5.93 \mathrm{~min}, \mathrm{t}_{\mathrm{R}}$ minor $=6.90 \mathrm{~min})$ 
Melting point: $132-136^{\circ} \mathrm{C}$
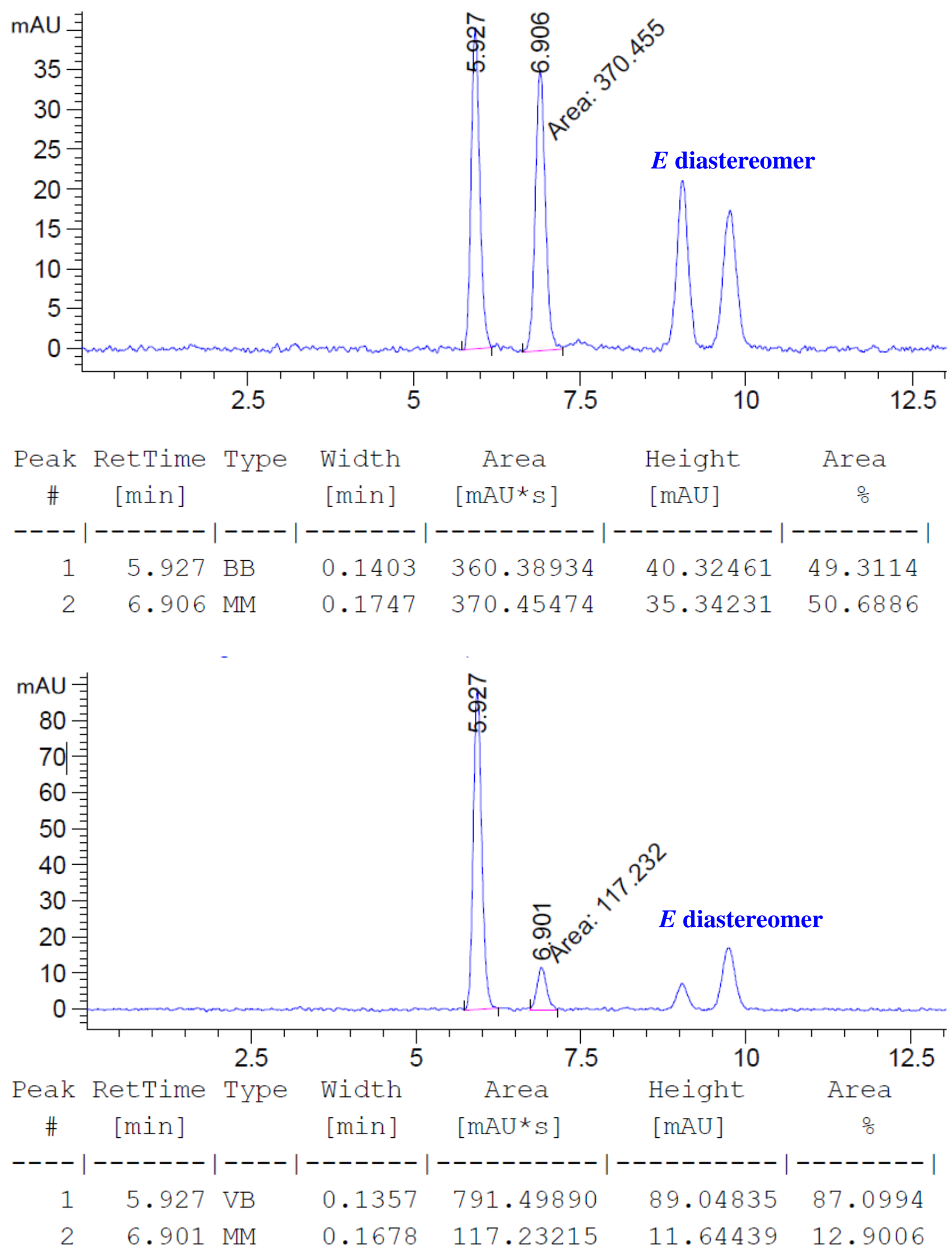


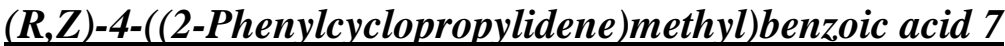<smiles>COC(=O)c1ccc(/C=C2\CC2c2ccccc2)cc1</smiles><smiles>CO[N+](=O)[O-]</smiles>

To a suspension of the major enantiomer of (Z)-methyl 4-((2-phenylcyclopropylidene)methyl)benzoate (Z)-6fa (0.05 mmol, 1.00 equiv., >99:1 er) in $\mathrm{MeOH}(168 \mu \mathrm{L}, 0.3 M)$ was added $\mathrm{NaOH}$ aq. solution (167 $\mu \mathrm{L}, 20.0$ equiv., 6.0M). The mixture was stirred at room temperature for 1 hour, whereby TLC indicated disappearance of starting material. The mixture was acidified with $\mathrm{HCl}$ aq. solution $(2.00 \mathrm{M}, 0.50 \mathrm{~mL}$, 20.0 equiv.) until a white solid started crashing out. At this point, the mixture was diluted with EtOAc, layers were separated and the aqueous layer was washed with EtOAc $(2 \times)$. The combined organics were dried over $\mathrm{MgSO}_{4}$, filtered and concentrated under reduced pressure to afford pure 7. X-ray structural analysis allowed to determine the absolute configuration as $(R)$ (see section $\underline{\text { Absolute configuration }}$ determination of 7 below).

Note1: A sample of the major enantiomer of (Z)-6fa was obtained by preparative HPLC (Chiralpak IB, Hexane/IPA $=99.5 / 0.5,18.0 \mathrm{~mL} / \mathrm{min}$ ).

Note2: No racemization was observed upon hydrolysis of $(Z)-6 \mathbf{f a}$ to the corresponding acid 7. (see section HPLC traces of rac-7 and (R)-7 below).

Yield: $>99 \%$

Appearance: colourless needle-shaped crystals

${ }^{1}$ H NMR (400 MHz, Chloroform-d): $\delta 7.95$ (d, $\left.J=8.5 \mathrm{~Hz}, 2 \mathrm{H}\right), 7.44(\mathrm{~d}, J=8.4 \mathrm{~Hz}, 2 \mathrm{H}), 7.29-7.23$ $(\mathrm{m}, 2 \mathrm{H}), 7.23-7.14(\mathrm{~m}, 1 \mathrm{H}), 7.13-7.06(\mathrm{~m}, 2 \mathrm{H}), 7.02-6.96(\mathrm{~m}, 1 \mathrm{H}), 2.96(\mathrm{ddd}, J=9.5,5.2,2.0 \mathrm{~Hz}$, $1 \mathrm{H}), 1.90(\mathrm{td}, J=9.5,2.0 \mathrm{~Hz}, 1 \mathrm{H}), 1.33$ (ddd, $J=9.5,5.2,2.0 \mathrm{~Hz}, 1 \mathrm{H})$.

${ }^{13}$ C NMR (101 MHz, Chloroform-d): $\delta$ 170.68, 142.60, 140.54, 132.13, 130.64, 128.84, 127.34, 127.04, 126.45, 126.41, 119.52, 21.81, 13.92.

IR (ATR): $v\left(\mathrm{~cm}^{-1}\right)$ 3028, 2966, 2669, 2545, 1687, 1605, 1427, 1315, 1289, 696.

HRMS (Nano-CHIP ESI/LTQ-Orbitrap): calcd for $\mathrm{C}_{17} \mathrm{H}_{13} \mathrm{O}_{2}^{-}$[M-H] $]^{-}$: 250.0994; found: 249.0918 . $[\boldsymbol{\alpha}]_{\mathbf{D}}^{\mathbf{2 0}}=-643\left(c=0.2, \mathrm{CHCl}_{3}\right)$.

e.r. $(\boldsymbol{Z})$ : $>99: 1$ (Chiralpak IB, Hexane/IPA $=99 / 10, \lambda=282 \mathrm{~nm}, 1.00 \mathrm{~mL} / \mathrm{min}, \mathrm{t}_{\mathrm{R}}$ major $=5.74 \mathrm{~min}$ )

Melting point: $149-151^{\circ} \mathrm{C}$ 


\section{Absolute configuration determination of 7:}

Carboxylic acid 7 (10.0 mg) was dissolved in $50.0 \mu \mathrm{L}$ of EtOAc and crystallized by slow diffusion of pentane. Crystals formed after 2 days at room temperature. X-Ray analysis confirmed the absolute stereochemistry of $\mathbf{7}$ as the $(R)$-enantiomer. Flack parameter $=0.05(6)$.

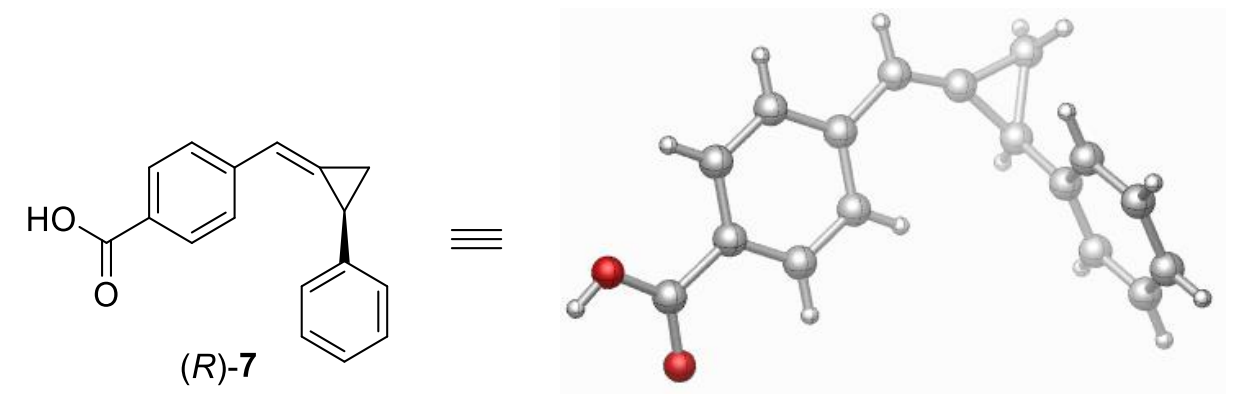

Ortep-represention of $(R)-7$ (thermal ellipsoids set at 50\% probability). CCDC1998995 contains the crystallographic data for $(R)-7$. These data can be obtained free of charge from The Cambridge Crystallographic Data Centre via www.ccdc.cam.ac.uk/data_request/cif. 


\begin{tabular}{|c|c|}
\hline Compound & 7 \\
\hline Empirical Formula & $\mathrm{C}_{17} \mathrm{H}_{14} \mathrm{O}_{2}$ \\
\hline$D_{\text {calc. }} / \mathrm{g} \mathrm{cm}^{-3}$ & 1.263 \\
\hline$\mu / \mathrm{mm}^{-1}$ & 0.652 \\
\hline Formula Weight & 250.28 \\
\hline Colour & clear colourless \\
\hline Shape & needle \\
\hline Size $/ \mathrm{mm}^{3}$ & $0.76 \times 0.10 \times 0.07$ \\
\hline$T / \mathrm{K}$ & $140.00(10)$ \\
\hline Crystal System & orthorhombic \\
\hline Flack Parameter & $0.05(6)$ \\
\hline Hooft Parameter & $0.06(5)$ \\
\hline Space Group & $P 2{ }_{1} 22_{1}$ \\
\hline$a / \AA ̊$ & $6.78088(5)$ \\
\hline$b / \AA$ & $17.69818(12)$ \\
\hline$c / \AA ̊$ & $21.93751(14)$ \\
\hline$\alpha /^{\circ}$ & 90 \\
\hline$\beta 1^{\circ}$ & 90 \\
\hline$\gamma 1^{\circ}$ & 90 \\
\hline $\mathrm{V} / \AA^{3}$ & $2632.70(3)$ \\
\hline$Z$ & 8 \\
\hline$Z^{\prime}$ & 2 \\
\hline Wavelength/Å & 1.54184 \\
\hline Radiation type & $\mathrm{Cu} K \alpha$ \\
\hline$\Theta_{\min } /^{\circ}$ & 3.208 \\
\hline$\Theta_{\max } /^{\circ}$ & 72.702 \\
\hline Measured Refl's. & 48731 \\
\hline Ind't Refl's & 5208 \\
\hline Refl's with I > 2(I) & 5037 \\
\hline$R_{\text {int }}$ & 0.0419 \\
\hline Parameters & 344 \\
\hline Restraints & 0 \\
\hline Largest Peak/e $\AA^{-3}$ & 0.179 \\
\hline Deepest Hole $/ \mathrm{e} \AA^{-3}$ & -0.195 \\
\hline GooF & 1.024 \\
\hline$w R 2$ (all data) & 0.0832 \\
\hline$w R_{2}$ & 0.0820 \\
\hline$R_{1}$ (all data) & 0.0311 \\
\hline$R_{1}$ & 0.0299 \\
\hline
\end{tabular}




\section{HPLC traces of rac-7 and (R)-7:}
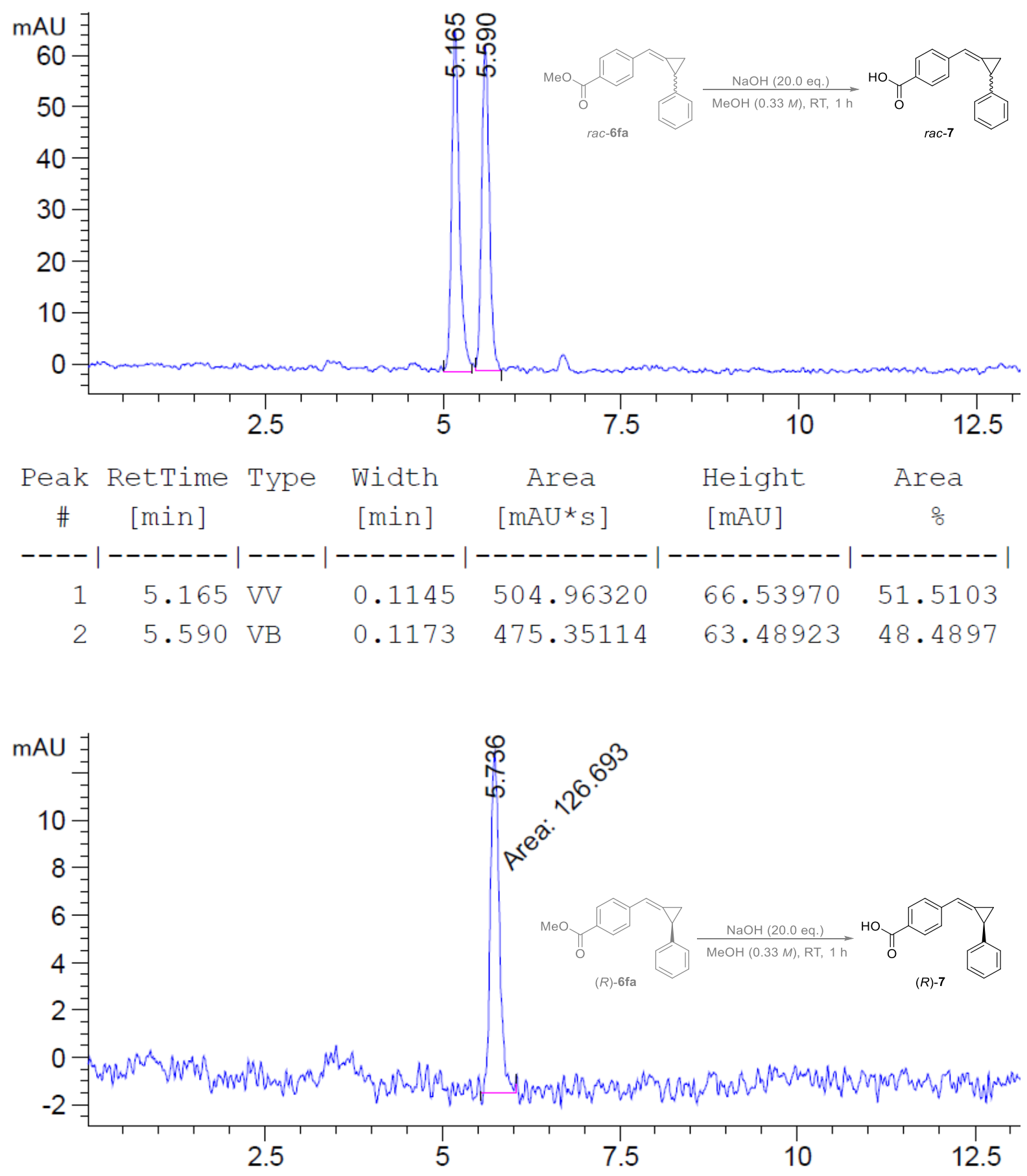


\section{Methyl 4-(((1R,2R)-2-phenylcyclopropyl)methyl)benzoate 8}

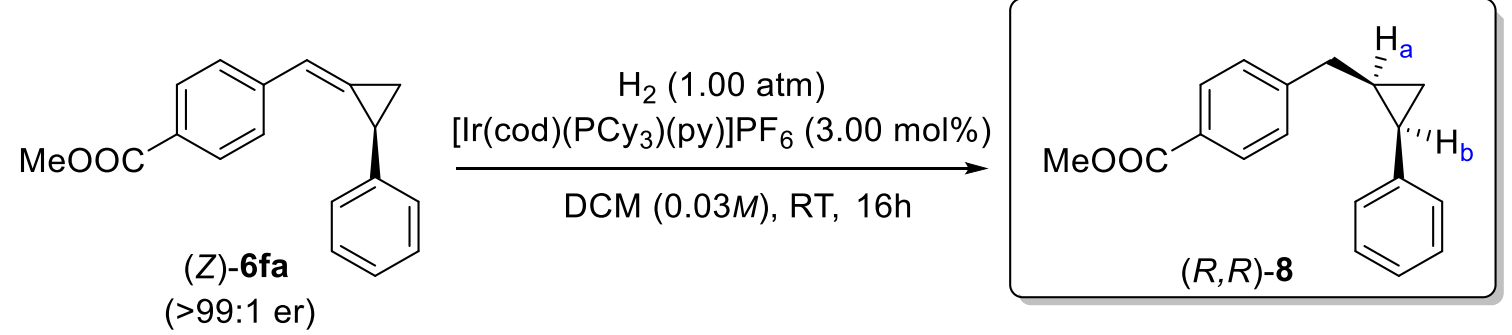

A sealed vial containing a solution of $(R, Z)$-methyl 4-((2-phenylcyclopropylidene)methyl)benzoate $(R)$ 6fa (0.05 mmol, 1.00 equiv., >99:1 er) in DCM (1.00 mL) was equipped with a $\mathrm{H}_{2}$ baloon. A solution of Crabrtree's catalyst $(1.5 \mu \mathrm{mol}, 3.00 \mathrm{~mol} \%)$ in DCM $(502 \mu \mathrm{L})$ was added to the reaction vessel. The solution was stirred at room temperature for 16 hours, whereby TLC indicated disappearance of starting material. The mixture was filtered over a silica plug (eluting with DCM) and concentrated under reduced pressure. Purification of the crude product from impurities via preparative HPLC (Purification conditions: Chiralpak IB, Hexane $/ \mathrm{IPA}=98 / 2, \lambda=254 \mathrm{~nm}, 18.0 \mathrm{~mL} / \mathrm{min})$ yielded pure $(R, R)-\mathbf{8}$.

Determination of the relative stereochemistry: the relative stereochemistry was assigned to be cis by the presence of ${ }^{1} \mathrm{H}$ NOE correlation between $\mathrm{H}_{\mathrm{a}}$ and $\mathrm{H}_{\mathrm{b}}$.

Note1: A sample of the major enantiomer of (Z)-6fa was obtained by preparative HPLC (Chiralpak IB, Hexane/IPA $=99.5 / 0.5,18.0 \mathrm{~mL} / \mathrm{min})$.

Note2: No enantioenrichment of $(R, R)-\mathbf{8}$ was obtained from purification of the product via preparative

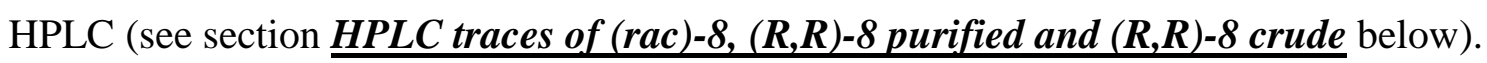

\section{Yield: $85 \%$}

Appearance: colourless oil

${ }^{1}$ H NMR (400 MHz, Benzene-d6): $\delta 8.09(\mathrm{~d}, J=8.3 \mathrm{~Hz}, 2 \mathrm{H}), 7.17-6.99(\mathrm{~m}, 5 \mathrm{H}), 6.88(\mathrm{~d}, J=8.3 \mathrm{~Hz}$, 2H), 3.50 (s, 3H), 2.30 (dd, $J=15.2,6.7 \mathrm{~Hz}, 1 \mathrm{H}), 2.07$ (dd, $J=15.3,7.8 \mathrm{~Hz}, 1 \mathrm{H}), 1.95$ (td, $J=8.6,6.0$ $\mathrm{Hz}, 1 \mathrm{H}), 1.14-0.97(\mathrm{~m}, 1 \mathrm{H}), 0.81-0.70(\mathrm{~m}, 1 \mathrm{H}), 0.65-0.56(\mathrm{~m}, 1 \mathrm{H})$.

${ }^{13}$ C NMR (101 MHz, Chloroform-d): $\delta$ 167.34, 147.72, 138.90, 129.64, 129.23, 128.45, 128.14, 127.83, $126.11,52.09,34.67,21.45,19.61,9.92$.

IR (ATR): $v\left(\mathrm{~cm}^{-1}\right)$ 3062, 3026, 3001, 2950, 2850, 1716, 1434, 1273, 1178, 1107, 1020, 753, 699.

HRMS (APPI/LTQ-Orbitrap): calcd for $\mathrm{C}_{18} \mathrm{H}_{18} \mathrm{O}_{2}{ }^{+}[\mathrm{M}]^{+}:$266.1301; found: 266.1300.

$[\boldsymbol{\alpha}] \mathbf{D}^{\mathbf{2 0}}=+21.7\left(c=0.1, \mathrm{CHCl}_{3}\right)$.

e.r.: 96:4 (Chiralpak ID, Hexane/IPA $=99 / 1, \lambda=254 \mathrm{~nm}, 1.00 \mathrm{~mL} / \mathrm{min}, \mathrm{t}_{\mathrm{R}}$ major $=9.74 \mathrm{~min}, \mathrm{t}_{\mathrm{R}}$ minor $=$ $8.93 \mathrm{~min})$ 


\section{HPLC traces of $($ rac $)-8,(R, R)-8$ purified and $(R, R)-8$ crude:}

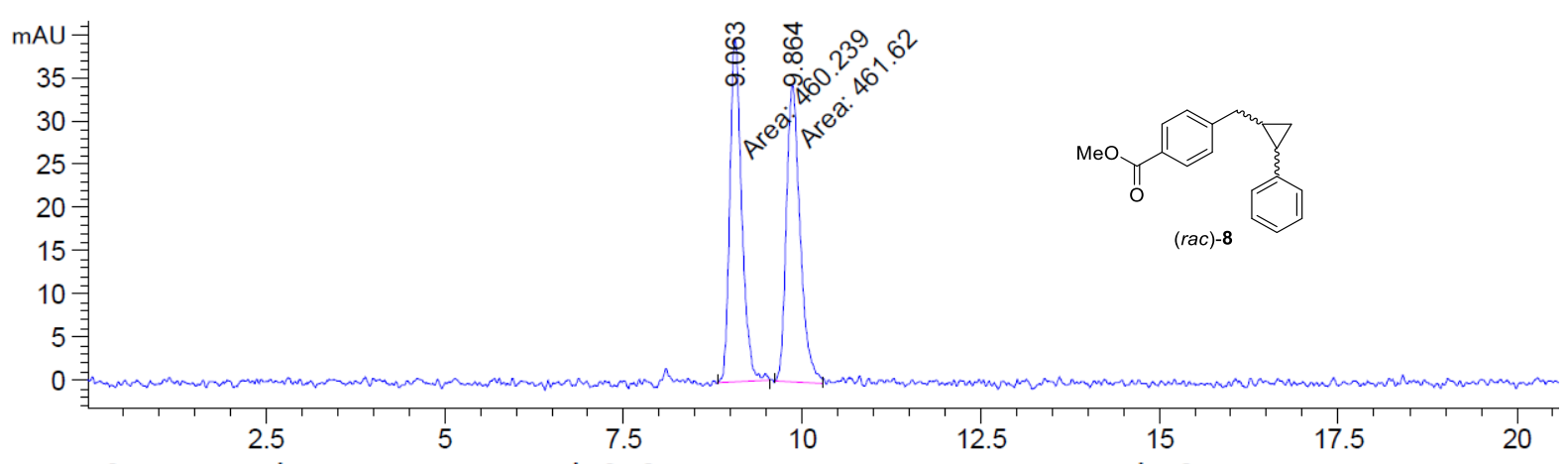

Peak RetTime Type Width Area Height Area

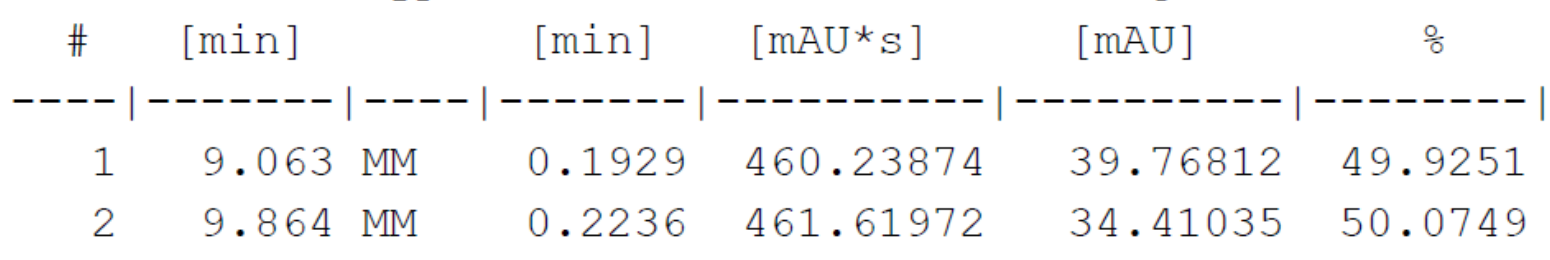

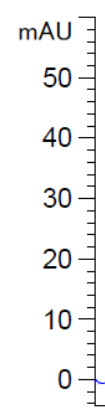

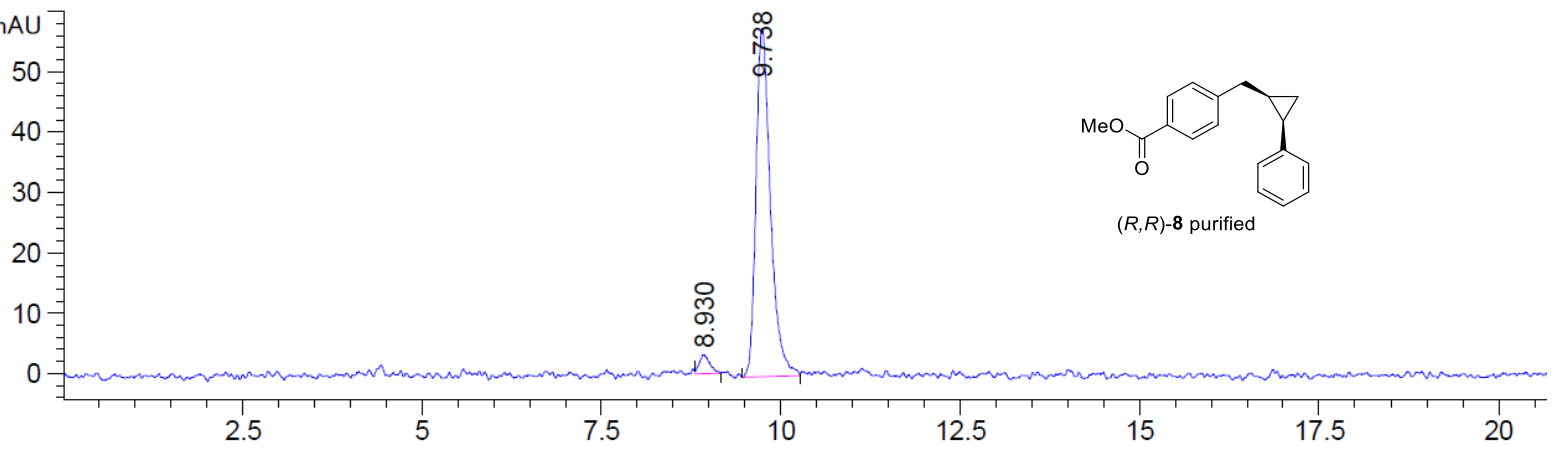

Peak RetTime Type Width Area Height Area

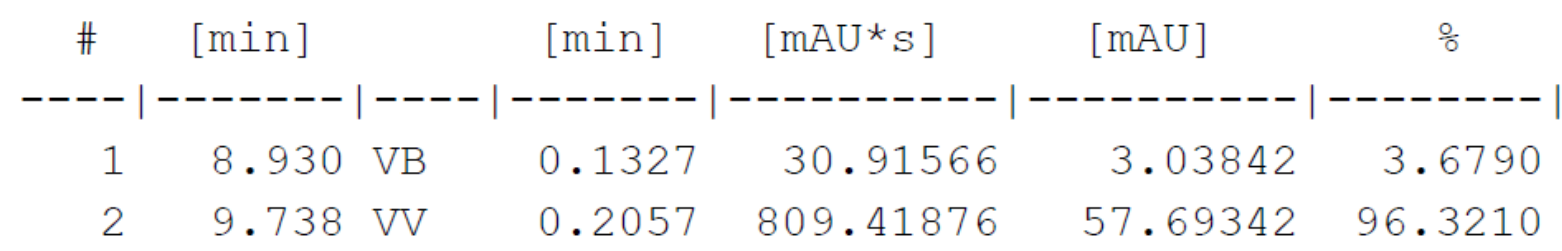
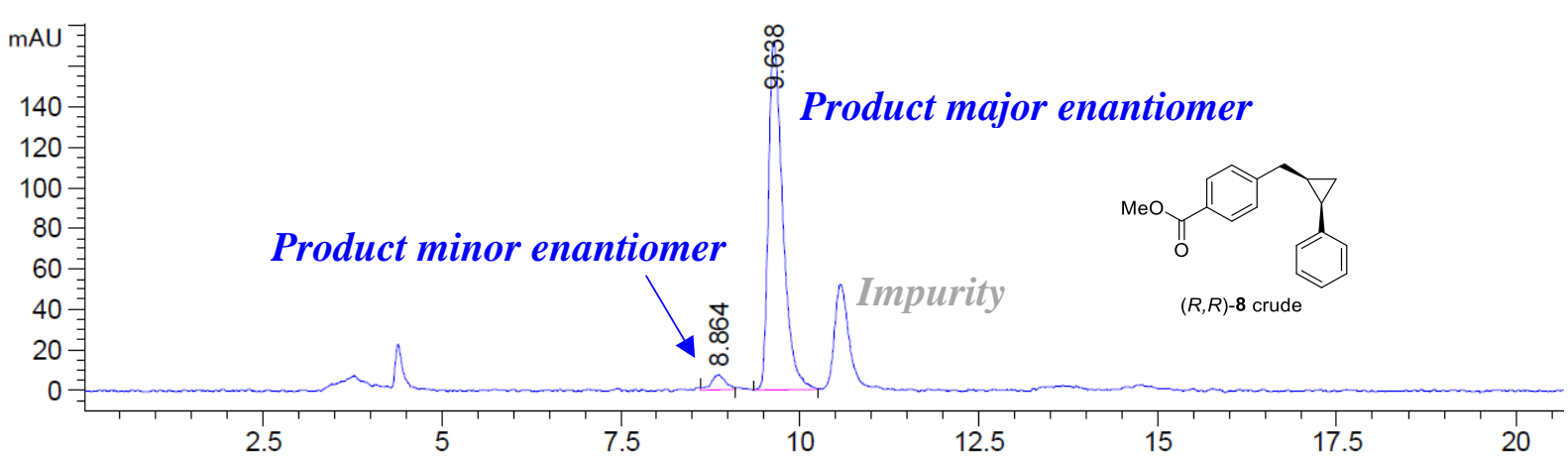

Peak RetTime Type Width Area Height Area

\begin{tabular}{|c|c|c|c|c|c|}
\hline \# & [min ] & {$[\mathrm{min}]$} & {$[\mathrm{mAU} * \mathrm{~s}]$} & [mAU] & $\frac{\circ}{0}$ \\
\hline & & & & & \\
\hline 1 & $8.864 \mathrm{VV}$ & 1748 & 99.78941 & 23858 & 993 \\
\hline 2 & $9.638 \mathrm{BV}$ & 0.2062 & 2389.18530 & 171.80786 & 95 \\
\hline
\end{tabular}




\section{Methyl (R,E)-4-(3-phenylbut-1-en-1-yl)benzoate 9}
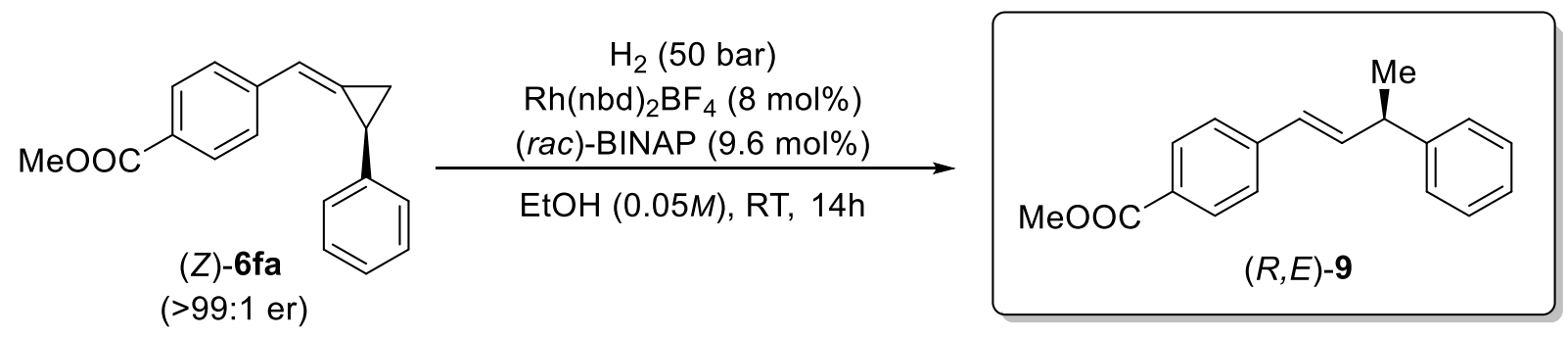

In a flame dried vial and under a $\mathrm{N}_{2}$ atmosphere, $\left[\mathrm{Rh}(\mathrm{nbd})_{2}\right] \mathrm{BF}_{4}(1.82 \mu \mathrm{mol}, 8.00 \mathrm{~mol} \%)$ and $(\mathrm{rac})-2,2^{\prime}-$ bis(diphenylphosphino)-1,1'-binaphthalene ( $r a c)$-BINAP $(2.18 \mu \mathrm{mol}, 9.60 \mathrm{~mol} \%)$ were dissolved in EtOH $(354 \mu \mathrm{L})$ and stirred for $15 \mathrm{~min}$. Subsequently a solution of $(Z)-6 \mathbf{f a}(22.7 \mu \mathrm{mol}, 1.00$ equiv.) in EtOH $(100 \mu \mathrm{L})$ was added. The vial was transferred into an autoclave and the reactor was purged three times with $\mathrm{H}_{2}$. The reactor was then pressurized to 50 bar with $\mathrm{H}_{2}$ and the mixture was stirred at ambient temperature for $14 \mathrm{~h}$. The reaction mixture was filtered over a plug of silica (eluting with $\mathrm{CH}_{2} \mathrm{Cl}_{2}$ ) and concentrated under reduced pressure. Purification of the crude product via preparative HPLC (Purification conditions: Chiralpak IC, Hexane/IPA $=99.5 / 0.05, \lambda=254 \mathrm{~nm}, 18.0 \mathrm{~mL} / \mathrm{min})$ yielded pure $(R, E)-9$. Analytical data are in accordance with literature. ${ }^{[1]}$ The other product of the reaction is $(R)-\mathbf{1 0}$.

Note1: A sample of the major enantiomer of (Z)-6fa was obtained by preparative HPLC (Chiralpak IB, Hexane/IPA $=99.5 / 0.5,18.0 \mathrm{~mL} / \mathrm{min})$.

Note2: No enantioenrichment of $(R, E)-9$ was obtained from purification of the product via preparative HPLC (see section HPLC traces of rac-9, (R,E)-9 purified and $(\boldsymbol{R}, \boldsymbol{E})-9$ crude below).

Yield: $60 \%$

Appearance: colourless oil

${ }^{1}$ H NMR (400 MHz, Chloroform-d): $\delta 7.95$ (d, $\left.J=8.4 \mathrm{~Hz}, 2 \mathrm{H}\right), 7.40(\mathrm{~d}, J=8.4 \mathrm{~Hz}, 2 \mathrm{H}), 7.33$ (t, $J=7.6$ $\mathrm{Hz}, 2 \mathrm{H}), 7.30-7.20(\mathrm{~m}, 3 \mathrm{H}), 6.52(\mathrm{dd}, J=15.9,6.7 \mathrm{~Hz}, 1 \mathrm{H}), 6.44(\mathrm{~d}, J=16.2 \mathrm{~Hz}, 1 \mathrm{H}), 3.90$ (s, 3H), 3.67 (p, $J=6.9 \mathrm{~Hz}, 1 \mathrm{H}), 1.48(\mathrm{~d}, J=7.0 \mathrm{~Hz}, 3 \mathrm{H})$.

${ }^{13}$ C NMR (101 MHz, Chloroform-d): $\delta$ 167.12, 145.19, 142.24, 138.24, 130.01, 128.73, 128.61, 127.88, 127.44, 126.55, 126.15, 52.19, 42.86, 21.17.

$[\boldsymbol{\alpha}] \mathbf{D}^{\mathbf{2 0}}=+28.3\left(c=0.1, \mathrm{CHCl}_{3}\right)$.

e.r.: >99:1 (Chiralpak IC, Hexane/IPA $=99.5 / 0.5, \lambda=280 \mathrm{~nm}, 1.00 \mathrm{~mL} / \mathrm{min}, \mathrm{t}_{\mathrm{R}}$ major $=21.5 \mathrm{~min}$ ) 


\section{HPLC traces of (rac)-9, (R,E)-9 purified and (R,E)-9 crude:}

$\begin{aligned} & \mathrm{mAU}= \\ & 200 \\ & 150= \\ & 100= \\ & 50= \\ & 0= \\ & 0\end{aligned}$

Peak RetTime Type width

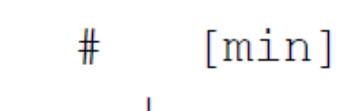

$$
-
$$

[min] (rac)-6fa

starting material

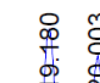

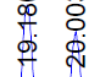

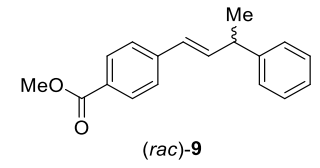

I

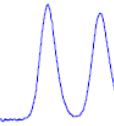

15

Area

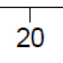
$119.180 \mathrm{BV}$
0.33335942 .52002
263.26355
50.2310
$220.003 \mathrm{VV}$
$0.3800 \quad 5887.87158$
241.83928
49.7690

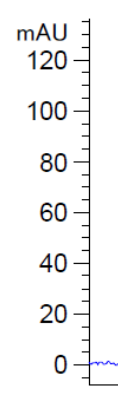

Peak RetTime Type

\# $[\mathrm{min}]$

$121.537 \mathrm{BV}$

0.3735

Width

Area

[min $\quad\left[\mathrm{mAU}{ }^{*} \mathrm{~s}\right]$

Height

Area

응

$$
\text { (n) }
$$

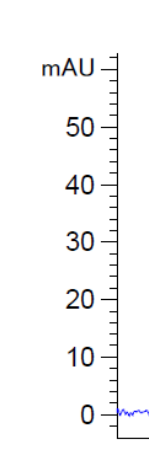

re

Peak RetTime Type Width

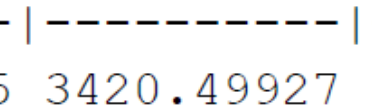

策

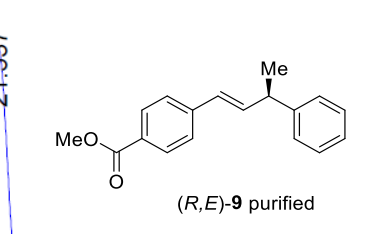

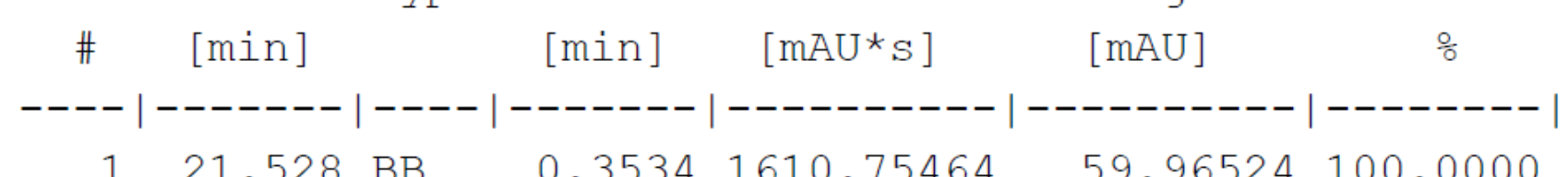




\section{$\underline{\operatorname{Methyl}(\mathrm{R})-4-(3-p h e n y l b u t y l) b e n z o a t e} 10$}
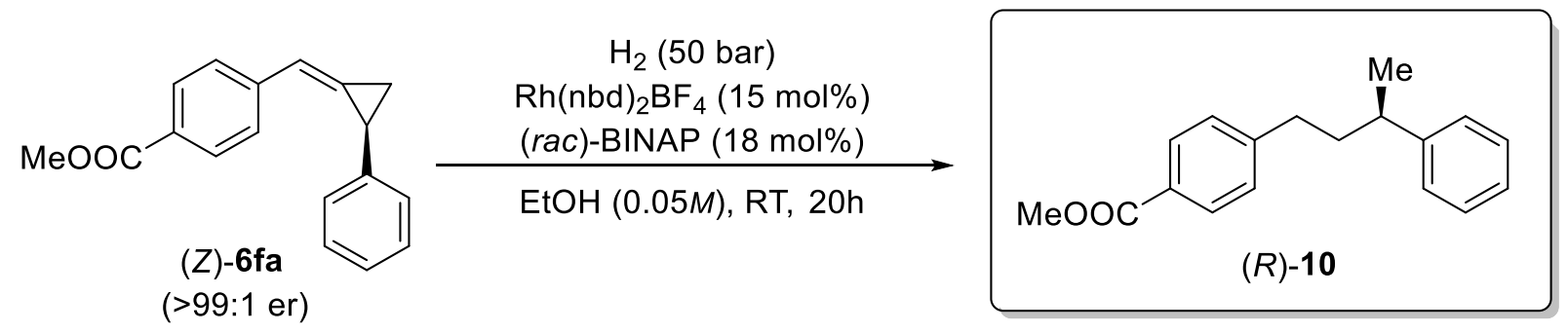

In a flame dried vial and under a $\mathrm{N}_{2}$ atmosphere, $\left[\mathrm{Rh}(\mathrm{nbd})_{2}\right] \mathrm{BF}_{4}(3.41 \mu \mathrm{mol}, 15.0 \mathrm{~mol} \%)$ and $(\mathrm{rac})-2,2^{\prime}-$ bis(diphenylphosphino)-1,1'-binaphthalene ( $r a c)$-BINAP (4.09 $\mu \mathrm{mol}, 18.0 \mathrm{~mol} \%)$ were dissolved in EtOH $(354 \mu \mathrm{L})$ and stirred for $15 \mathrm{~min}$. Subsequently a solution of $(Z)-6 \mathbf{f a}(22.7 \mu \mathrm{mol}, 1.00$ equiv.) in EtOH $(100 \mu \mathrm{L})$ was added. The vial was transferred into an autoclave and the reactor was purged three times with $\mathrm{H}_{2}$. The reactor was then pressurized to 50 bar with $\mathrm{H}_{2}$ and the mixture was stirred at ambient temperature for $20 \mathrm{~h}$. The reaction mixture was filtered over a plug of silica (eluting with $\mathrm{CH}_{2} \mathrm{Cl}_{2}$ ) and concentrated under reduced pressure. Purification of the crude via silica gel column chromatogreaphy $(n-$ pentane:EtOAc 98:2) yielded pure $(R)-\mathbf{1 0}$.

Note1: A sample of the major enantiomer of (Z)-6fa was obtained by preparative HPLC (Chiralpak IB, Hexane $/ \mathrm{IPA}=99.5 / 0.5,18.0 \mathrm{~mL} / \mathrm{min})$.

Yield: $90 \%$

Appearance: colourless oil

${ }^{1}$ H NMR (400 MHz, Chloroform-d): $\delta 7.93(\mathrm{~d}, J=8.2 \mathrm{~Hz}, 2 \mathrm{H}), 7.37$ - $7.28(\mathrm{~m}, 2 \mathrm{H}), 7.25$ - 7.13 (m, 5H), $3.90(\mathrm{~s}, 3 \mathrm{H}), 2.71(\mathrm{~h}, J=7.0 \mathrm{~Hz}, 1 \mathrm{H}), 2.64-2.49(\mathrm{~m}, 2 \mathrm{H}), 2.01-1.83(\mathrm{~m}, 2 \mathrm{H}), 1.28(\mathrm{~d}, J=7.0 \mathrm{~Hz}$, $3 \mathrm{H})$.

${ }^{13}$ C NMR (101 MHz, Chloroform-d): $\delta$ 167.31, 148.25, 147.04, 129.78, 128.60, 128.54, 127.82, 127.18, $126.23,52.11,39.70,39.66,34.15,22.63$.

IR (ATR): $v\left(\mathrm{~cm}^{-1}\right)$ 3061, 3027, 2953, 2924, 2853, 1720, 1435, 1277, 1179, 1108, 763, 700.

HRMS (APCI/QTOF): calcd for $\mathrm{C}_{18} \mathrm{H}_{21} \mathrm{O}_{2}{ }^{+}[\mathrm{M}+\mathrm{H}]^{+}:$: 269.1536; found: 269.1536.

$[\boldsymbol{\alpha}]_{\mathbf{D}^{\mathbf{2 0}}}=-14.2\left(c=0.2, \mathrm{CHCl}_{3}\right)$.

e.r.: >99:1 (Chiralpak IC, Hexane/IPA $=99.5 / 0.5, \lambda=254 \mathrm{~nm}, 1.00 \mathrm{~mL} / \mathrm{min}, \mathrm{t}_{\mathrm{R}}$ major $=13.0 \mathrm{~min}$ ) 


\section{SUPPORTING INFORMATION}

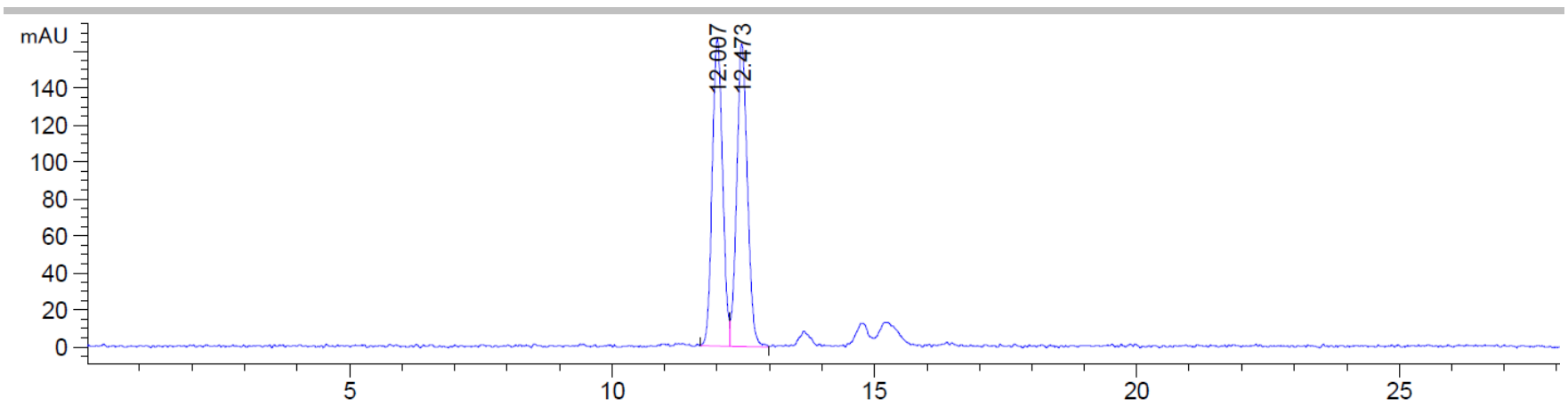

Peak RetTime Type width Area Height Area

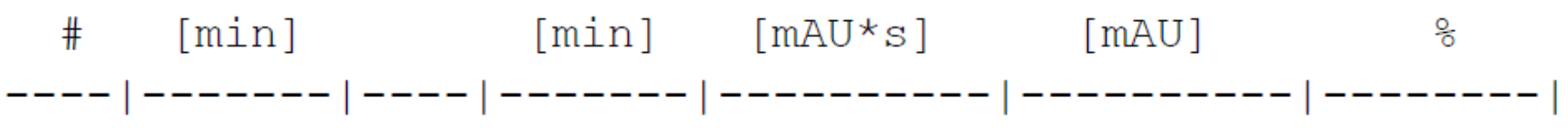

$1 \quad 12.007$ BV $\quad 0.2132 \quad 2273.18384 \quad 166.53427 \quad 49.3052$

$2 \quad 12.473$ VB $\quad 0.2182 \quad 2337.24707 \quad 164.11290 \quad 50.6948$

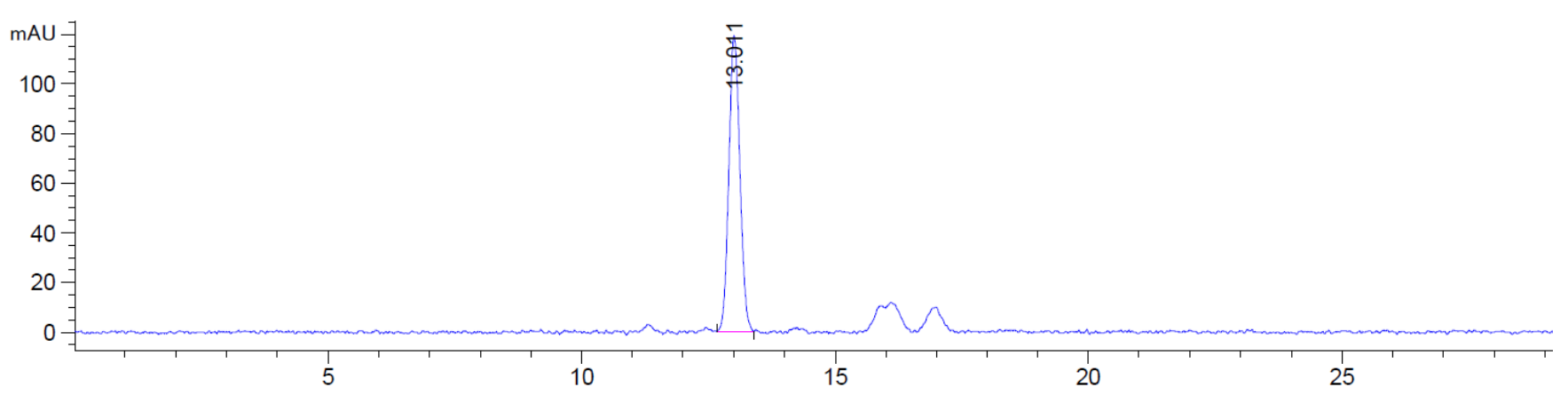

Peak RetTime Type width Area Height Area

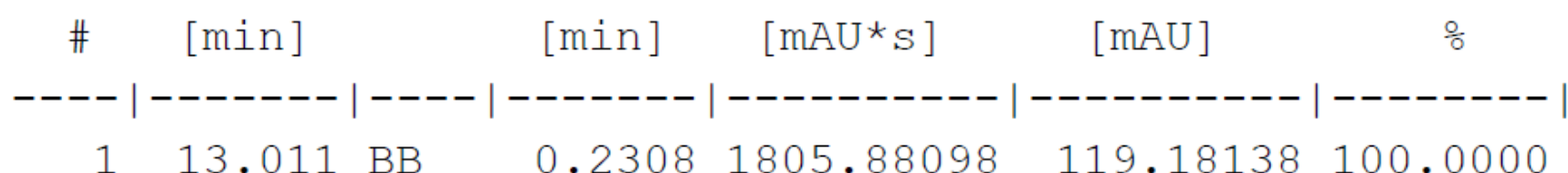




\section{X-ray crystallographic data}

$\underline{\left(R, R, N, N^{\prime} E, N, N^{\prime} E\right)-N, N^{\prime}-((1,8-N a p h t h y r i d i n e-2,7-d i v l) b i s(e t h a n-1-y l-1-y l i d e n e)) b i s(4-}$ methyl-2,6-bis((R)-1-phenylethyl)aniline) L1

$(R, R)-\mathbf{L} \mathbf{1}(40.0 \mathrm{mg})$ was dissolved in $100 \mu \mathrm{L}$ of EtOAc and crystallized by slow diffusion of pentane. Crystals formed after 4 days at room temperature.

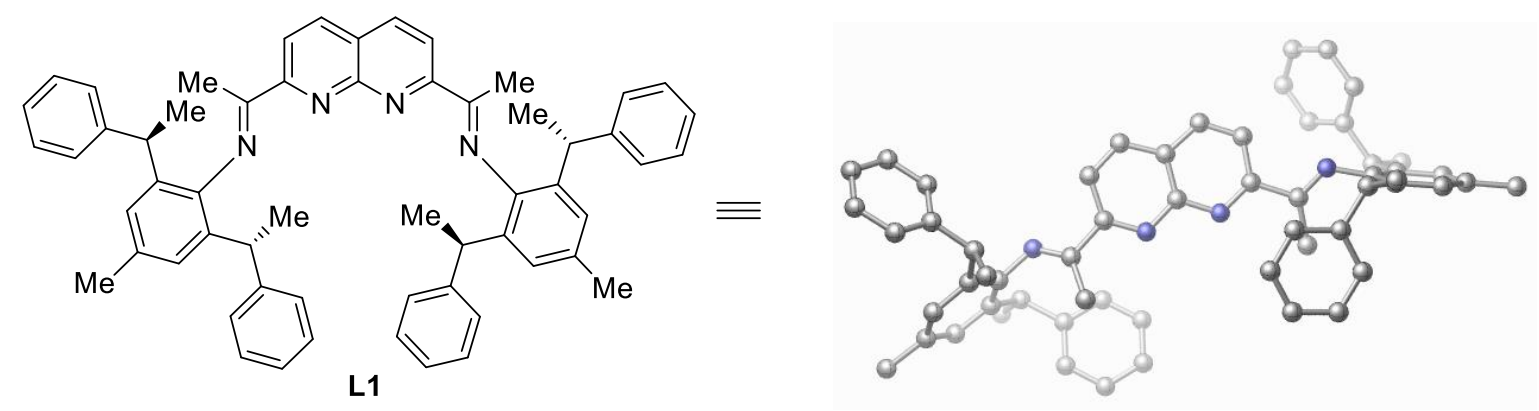

Ortep-represention of $(R, R)$-L1 (thermal ellipsoids set at 50\% probability). CCDC1998992 contains the crystallographic data for $(R, R)$-L1. These data can be obtained free of charge from The Cambridge Crystallographic Data Centre via www.ccdc.cam.ac.uk/data_request/cif. 


\begin{tabular}{|c|c|}
\hline Compound & L1 \\
\hline Empirical Formula & $\mathrm{C}_{58} \mathrm{H}_{56} \mathrm{~N}_{4}$ \\
\hline$D_{\text {calc. }} / \mathrm{g} \mathrm{cm}^{-3}$ & 1.154 \\
\hline$\mu / \mathrm{mm}^{-1}$ & 0.511 \\
\hline Formula Weight & 809.06 \\
\hline Colour & clear pale yellow \\
\hline Shape & plate \\
\hline Size $/ \mathrm{mm}^{3}$ & $0.23 \times 0.16 \times 0.06$ \\
\hline$T / \mathrm{K}$ & $140.00(10)$ \\
\hline Crystal System & monoclinic \\
\hline Flack Parameter & $0.2(5)$ \\
\hline Space Group & $P 2{ }_{1}$ \\
\hline$a / \AA ̊$ & $8.3188(2)$ \\
\hline$b / \AA ̊$ & $27.4105(9)$ \\
\hline$c / \AA ̊$ & $10.4707(3)$ \\
\hline$\alpha /^{\circ}$ & 90 \\
\hline$\beta /^{\circ}$ & $102.858(3)$ \\
\hline$\gamma /^{\circ}$ & 90 \\
\hline $\mathrm{V} / \AA^{3}$ & $2327.70(13)$ \\
\hline$Z$ & 2 \\
\hline$Z^{\prime}$ & 1 \\
\hline Wavelength/Å & 1.54184 \\
\hline Radiation type & $\mathrm{Cu} K \alpha$ \\
\hline$\Theta_{\min } /^{\circ}$ & 4.331 \\
\hline$\Theta_{\max } /^{\circ}$ & 75.471 \\
\hline Measured Refl's. & 21945 \\
\hline Ind't Refl's & 9215 \\
\hline 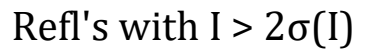 & 8144 \\
\hline$R_{\text {int }}$ & 0.0419 \\
\hline Parameters & 570 \\
\hline Restraints & 1 \\
\hline Largest Peak/e $\AA^{-3}$ & 0.161 \\
\hline Deepest Hole/e $\AA^{-3}$ & -0.149 \\
\hline GooF & 1.033 \\
\hline$w R_{2}$ (all data) & 0.1030 \\
\hline$w R_{2}$ & 0.0964 \\
\hline$R_{1}$ (all data) & 0.0503 \\
\hline$R_{1}$ & 0.0415 \\
\hline
\end{tabular}




\section{$\left(R, R, N, N^{\prime} E, N, N^{\prime} E\right)-N, N^{\prime}-((1,8-N a p h t h y r i d i n e-2,7-d i v l) b i s($ ethan-1-yl-1-ylidene $))$ bis(2,6-}

\section{bis((R)-1-(3,5-difluorophenyl)ethyl)-4-methylaniline) L9}

$(R, R)$-L9 (40.0 mg) was dissolved in $100 \mu \mathrm{L}$ of CPME and crystallized by slow diffusion of pentane. Crystals formed after 5 days at room temperature.<smiles>C#CC(=O)c1ccc2ccc(C(C)=Nc3c(C(C)c4cc(F)cc(F)c4)cc(C)cc3C(C)c3cc(F)cc(F)c3)nc2n1</smiles>

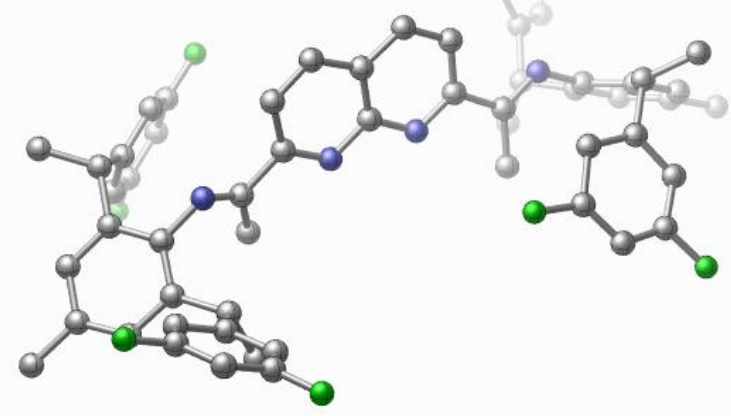

Ortep-represention of $(R, R)$-L9 (thermal ellipsoids set at 50\% probability). CCDC1998994 contains the crystallographic data for $(R, R)$-L9. These data can be obtained free of charge from The Cambridge Crystallographic Data Centre via www.ccdc.cam.ac.uk/data_request/cif. 


\begin{tabular}{|c|c|}
\hline Compound & L9 \\
\hline Empirical Formula & $\mathrm{C}_{58} \mathrm{H}_{48} \mathrm{~F}_{8} \mathrm{~N}_{4}$ \\
\hline$D_{\text {calc. }} / \mathrm{g} \mathrm{cm}^{-3}$ & 1.159 \\
\hline$\mu / \mathrm{mm}^{-1}$ & 0.726 \\
\hline Formula Weight & 953.00 \\
\hline Colour & clear intense yellow \\
\hline Shape & prism \\
\hline Size $/ \mathrm{mm}^{3}$ & $0.44 \times 0.33 \times 0.13$ \\
\hline$T / \mathrm{K}$ & $140.00(10)$ \\
\hline Crystal System & monoclinic \\
\hline Flack Parameter & $0.09(7)$ \\
\hline Space Group & $P 2{ }_{1}$ \\
\hline$a / \AA ̊$ & $11.8828(4)$ \\
\hline$b / \AA$ & $15.0104(3)$ \\
\hline$c / \AA ̊$ & $15.4953(4)$ \\
\hline$\alpha /^{\circ}$ & 90 \\
\hline$\beta /^{\circ}$ & $98.752(3)$ \\
\hline$\gamma 1^{\circ}$ & 90 \\
\hline $\mathrm{V} / \AA^{3}$ & 2731.64(13) \\
\hline$Z$ & 2 \\
\hline$Z^{\prime}$ & 1 \\
\hline Wavelength/Å & 1.54184 \\
\hline Radiation type & $\mathrm{Cu} K \alpha$ \\
\hline$\Theta_{\min } /^{\circ}$ & 2.885 \\
\hline$\Theta_{\max } /^{\circ}$ & 72.603 \\
\hline Measured Refl's. & 45337 \\
\hline Ind't Refl's & 10421 \\
\hline Refl's with I > 2(I) & 9742 \\
\hline$R_{\text {int }}$ & 0.0507 \\
\hline Parameters & 688 \\
\hline Restraints & 254 \\
\hline Largest Peak/e $\AA^{-3}$ & 0.429 \\
\hline Deepest Hole/e $\AA^{-3}$ & -0.212 \\
\hline GooF & 1.049 \\
\hline$w R_{2}$ (all data) & 0.1832 \\
\hline$w R_{2}$ & 0.1776 \\
\hline$R_{1}$ (all data) & 0.0655 \\
\hline$R_{1}$ & 0.0628 \\
\hline
\end{tabular}




\section{$\underline{{ }^{1} \mathrm{H},{ }^{13} \mathrm{C} \text { and }{ }^{19} \mathrm{~F} \text { NMR Spectra }}$}

\section{$\underline{\text { 2-Ethyl-1,8-naphthyridine } 3}$}

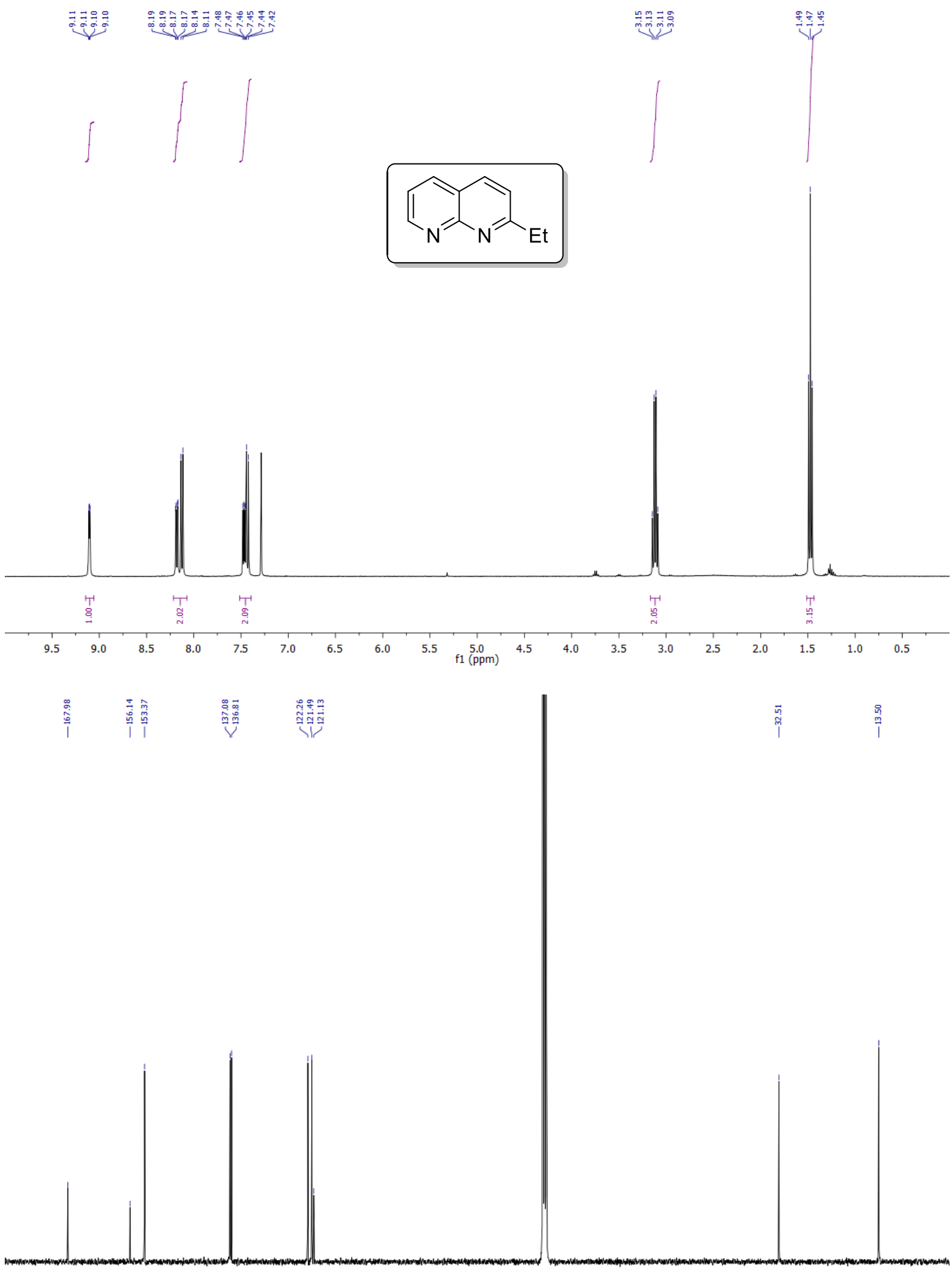

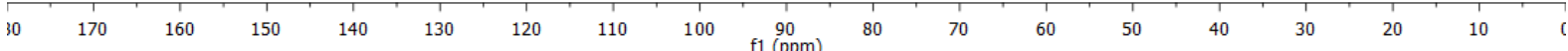




\section{2,7-diethyl-1,8-naphthyridine 11}
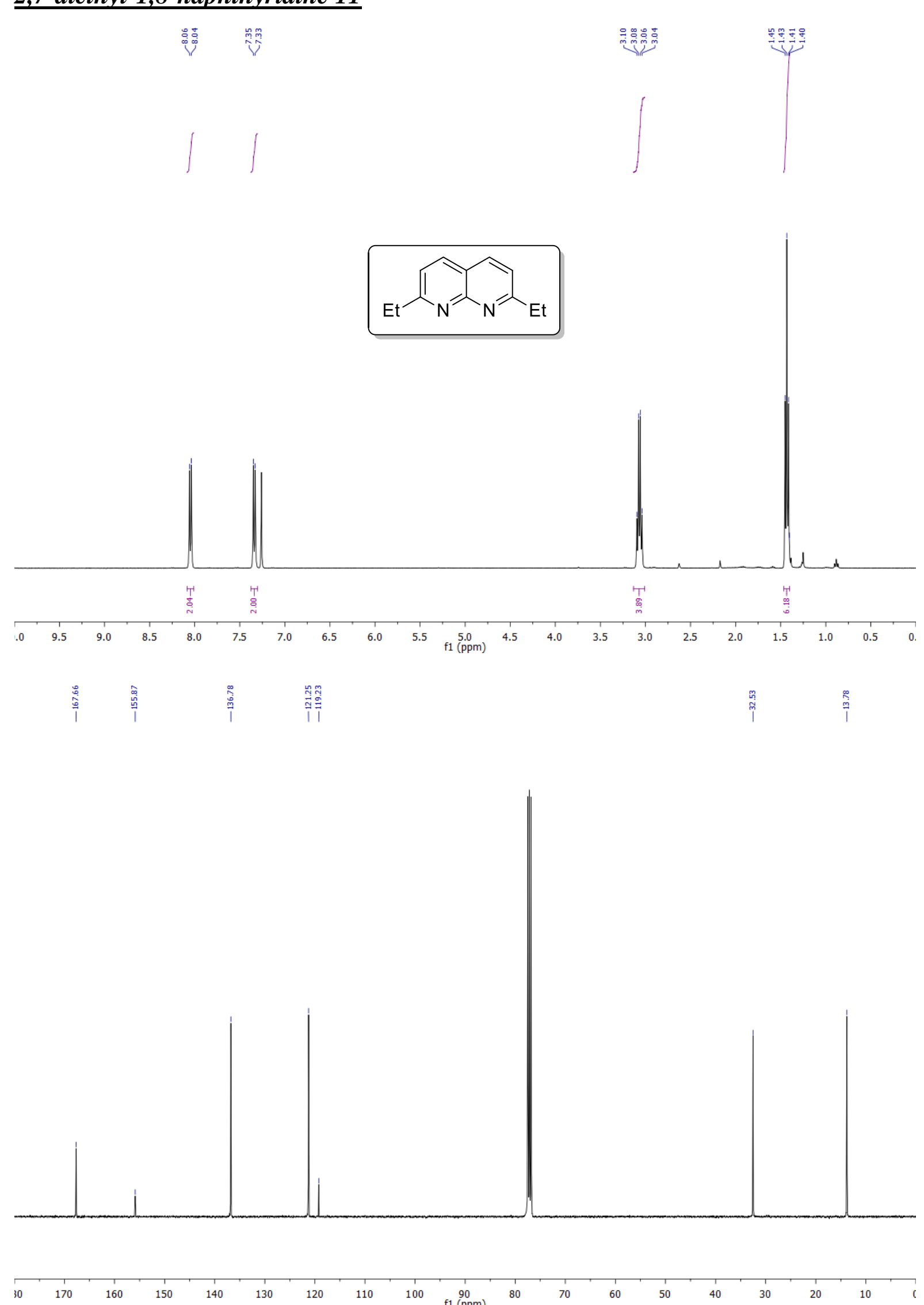
1,1'-(1,8-naphthyridine-2,7-divl)diethanone 2
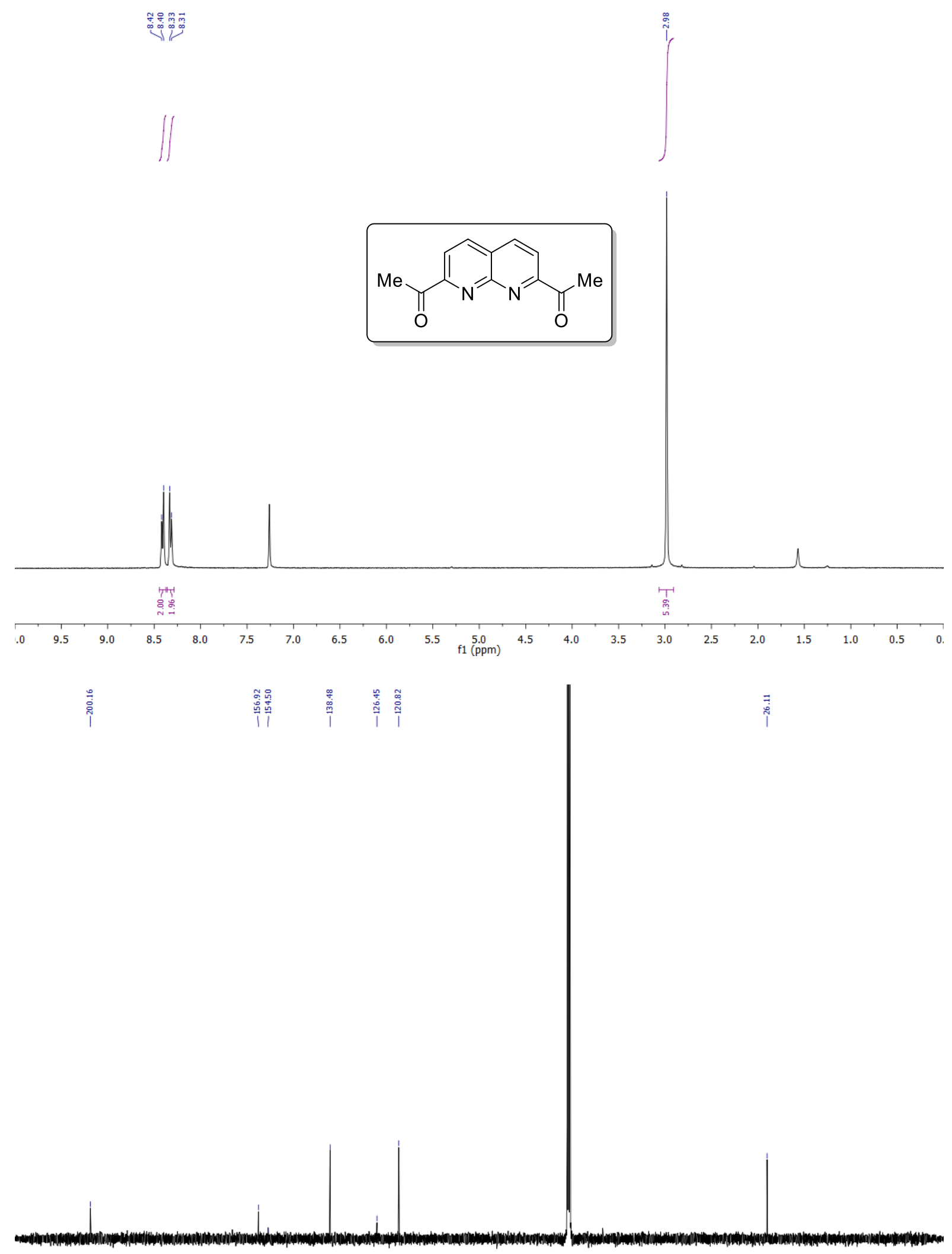

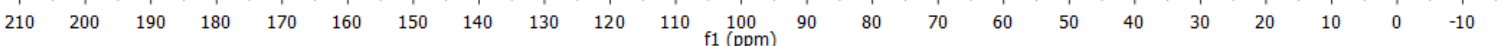




\section{$\underline{\text { 2-(1-(3,5-difluorophenyl)vinyl)-4,4,5,5-tetramethyl-1,3,2-dioxaborolane } 13}$}

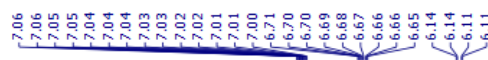
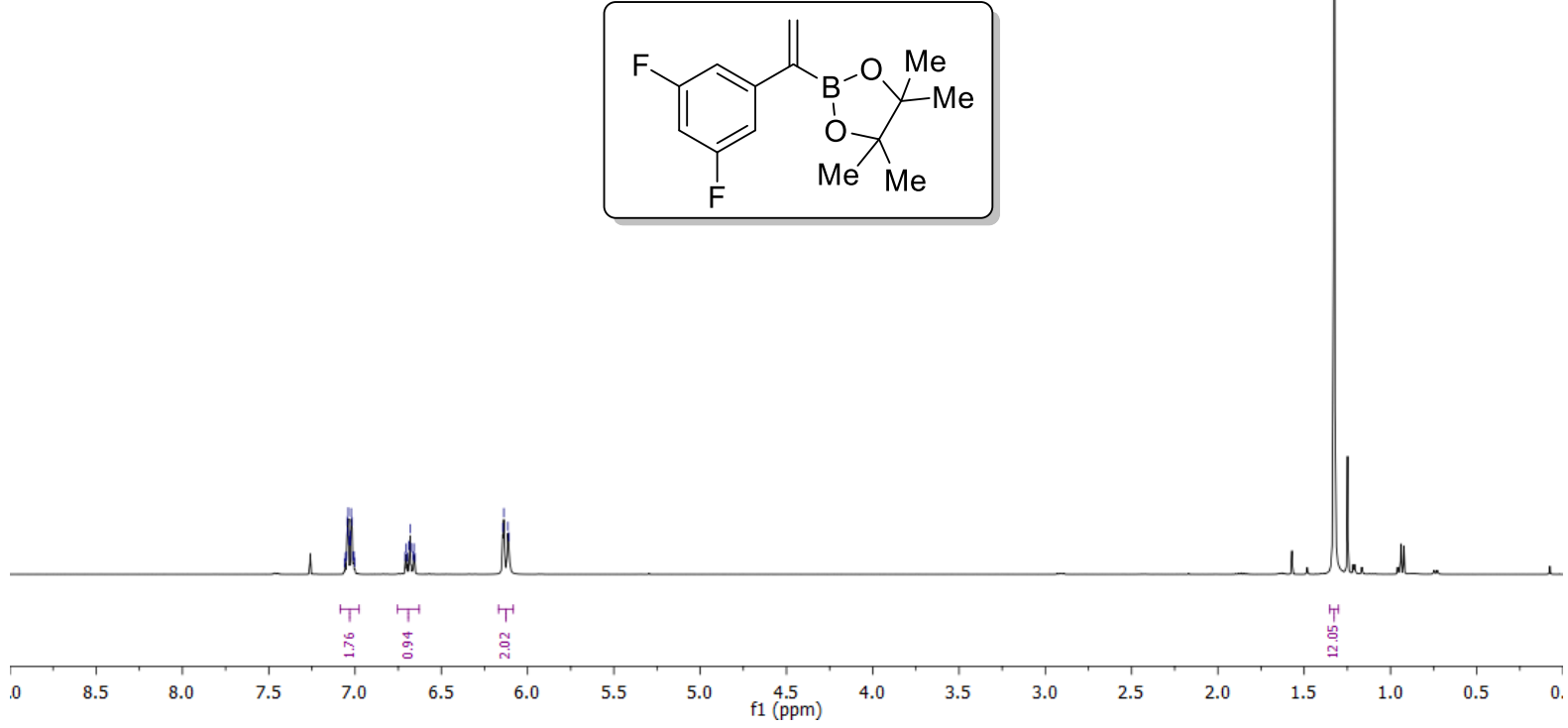

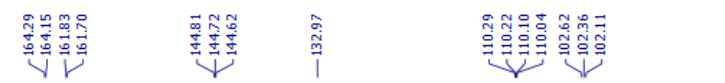

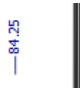

\section{4}

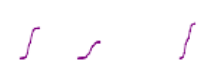

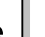

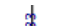


WILEY-VCH

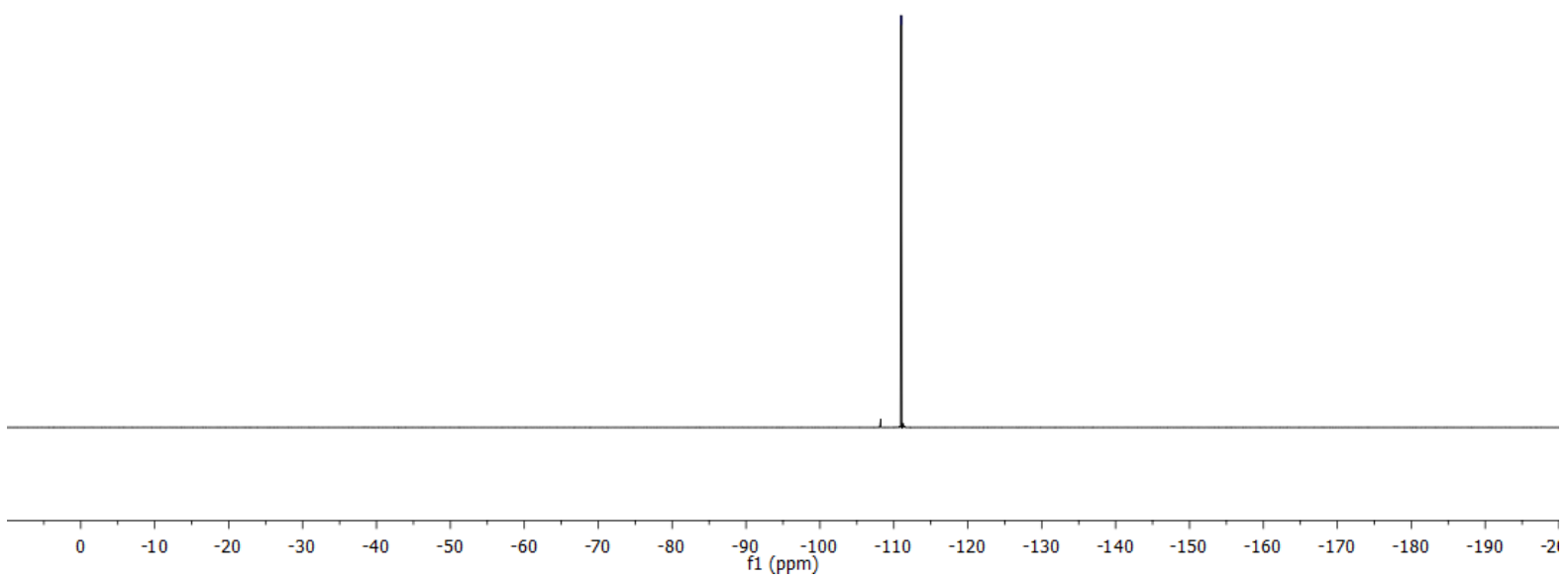




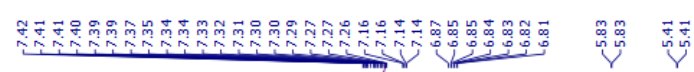

高
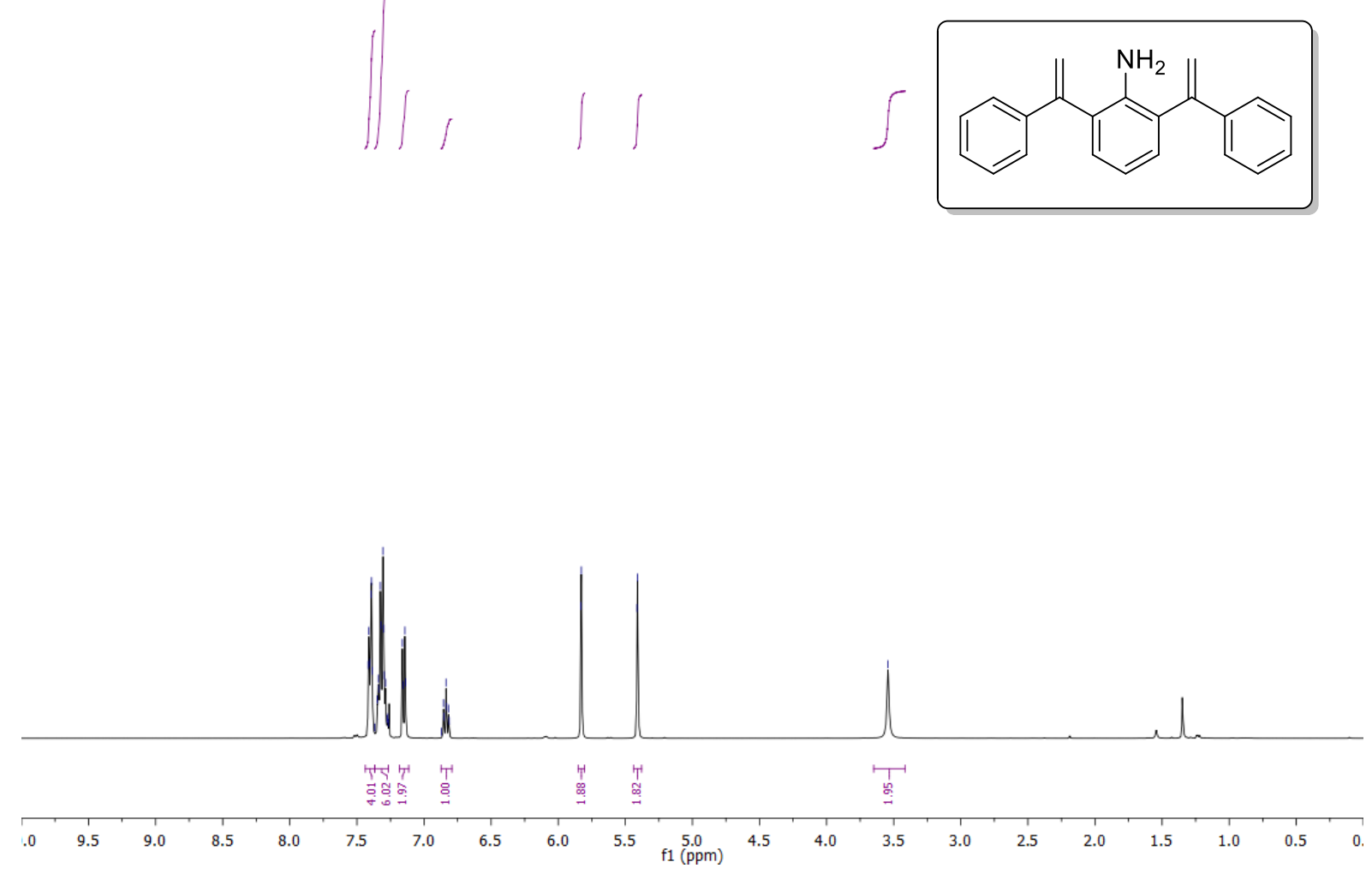

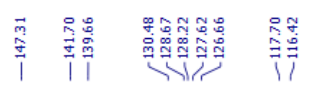

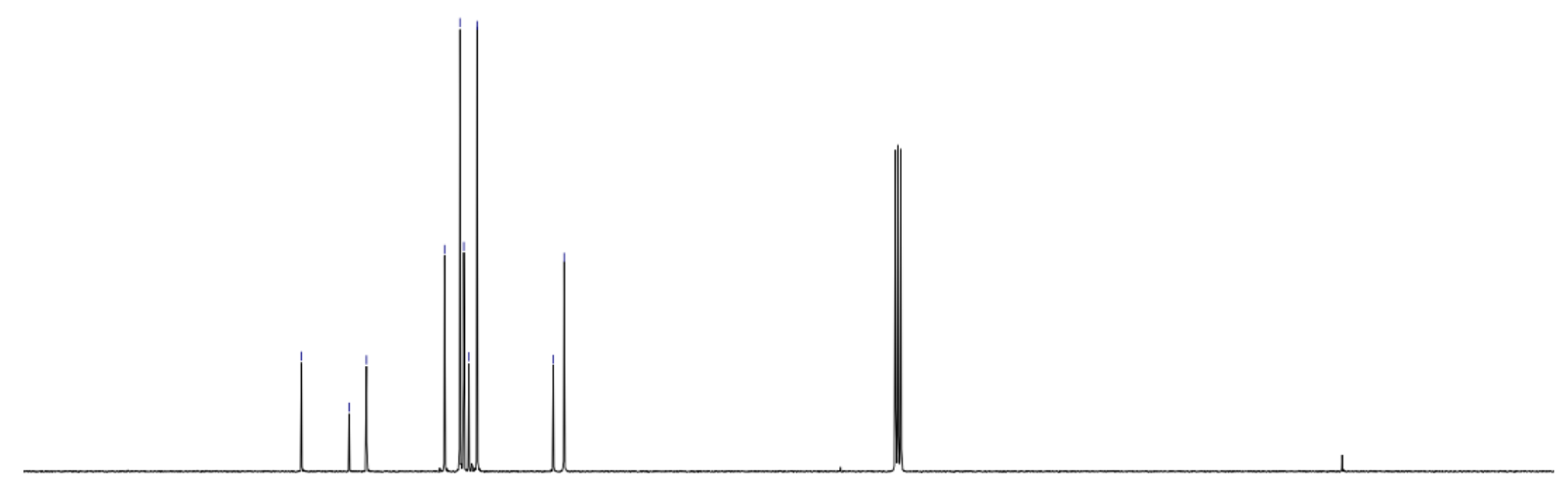




\section{2,6-bis(1-phenylvinyl)-4-(trifluoromethyl)aniline 12e}
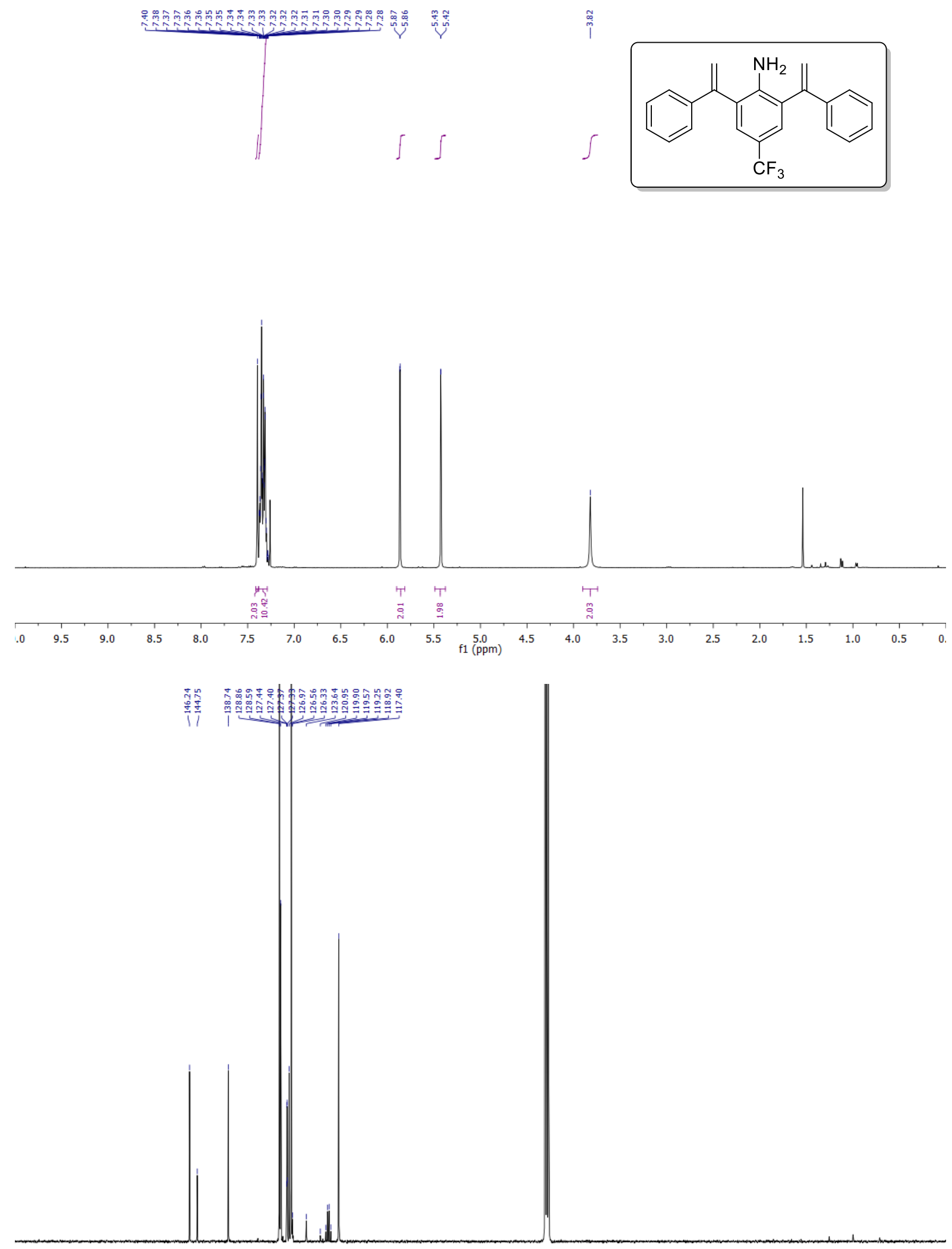
WILEY-VCH

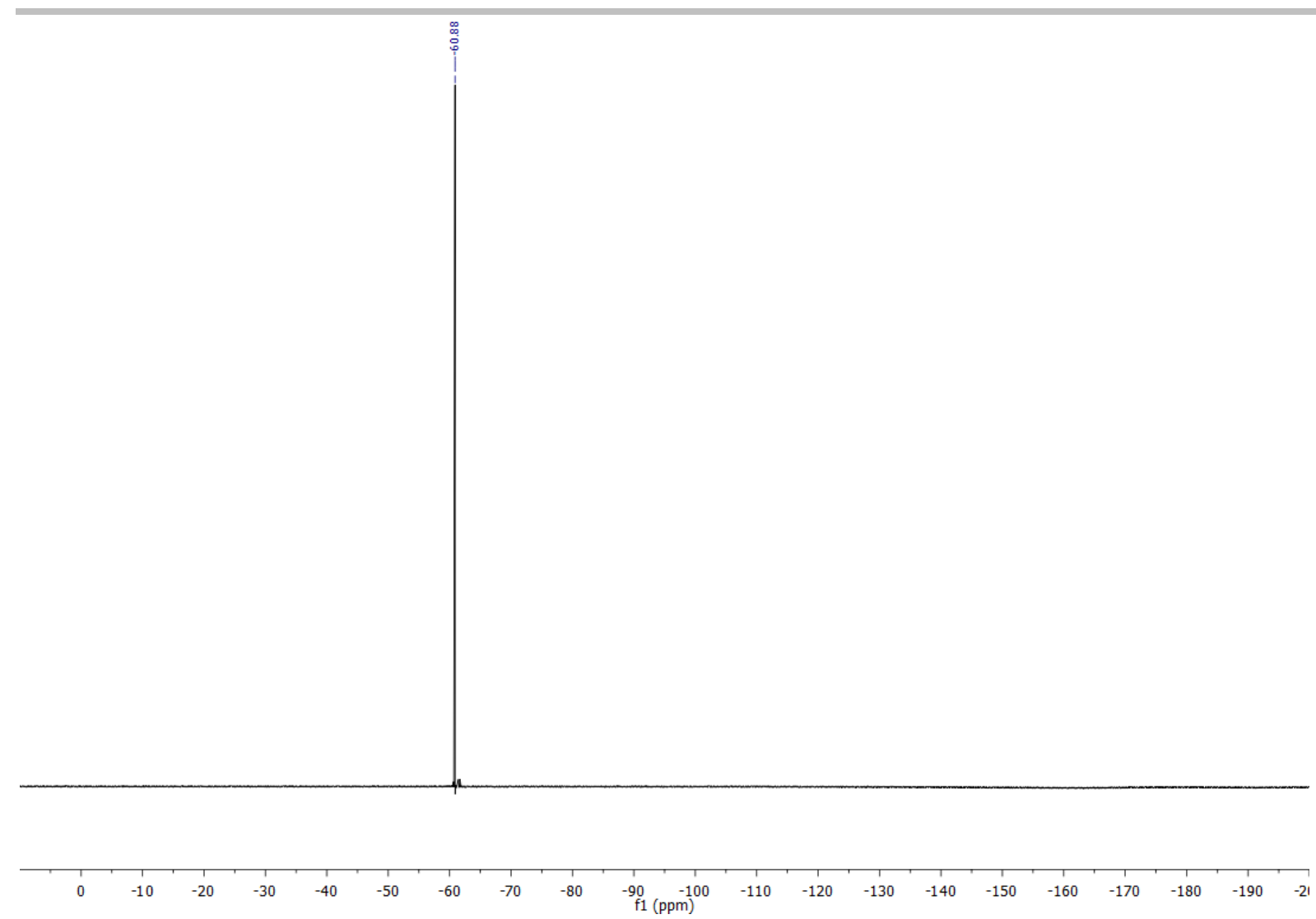




\section{2,6-bis(1-(4-(tert-butyl)phenyl)vinyl)-4-methylaniline 12f}

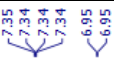

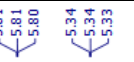

\section{สุ ฟิ}
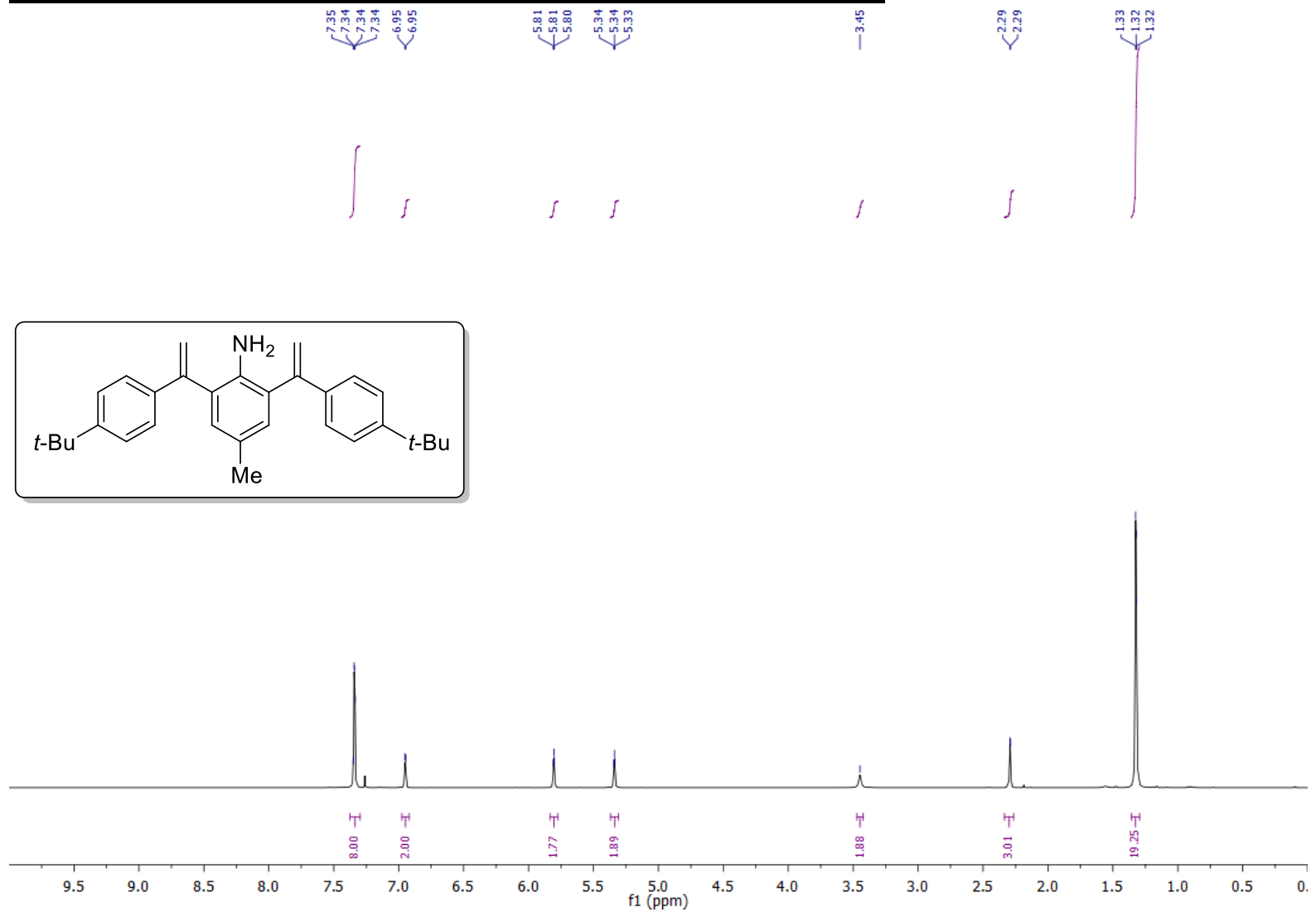

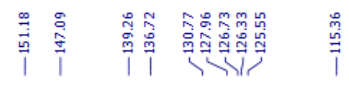

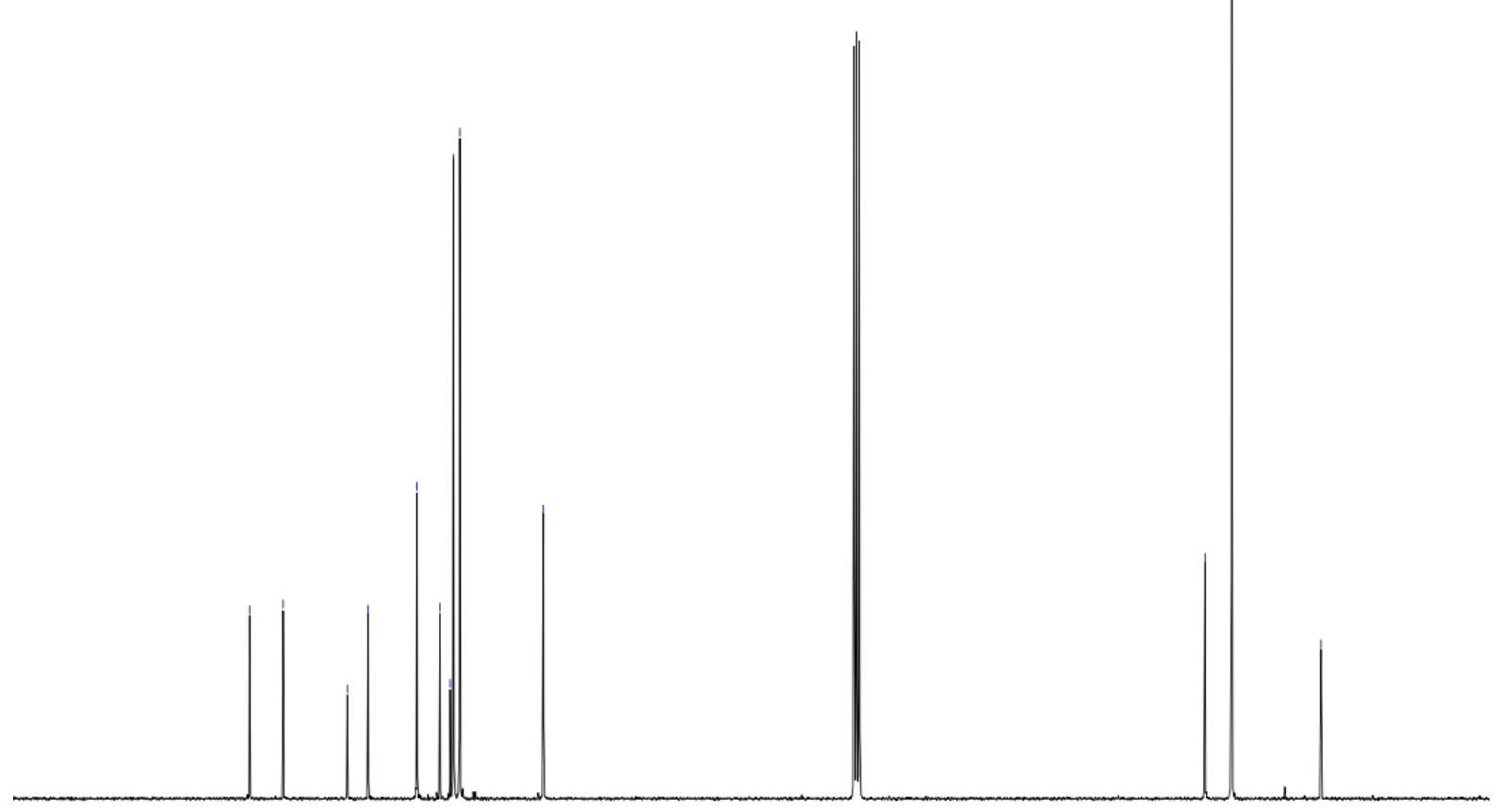

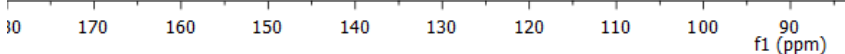




\section{4-methyl-2,6-bis(1-(naphthalen-2-yl)vinyl)aniline 12g}
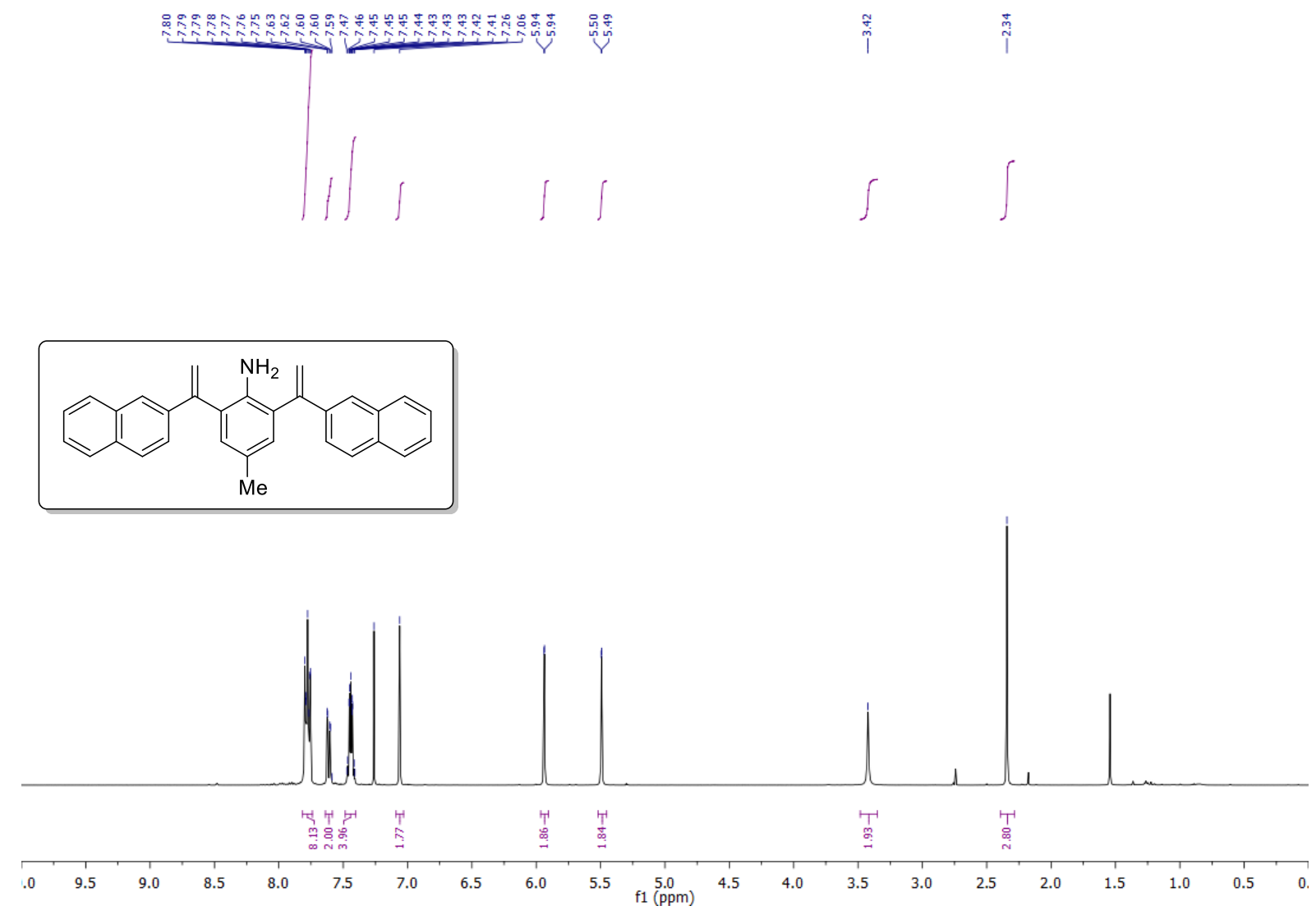

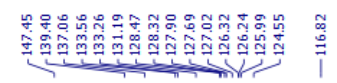

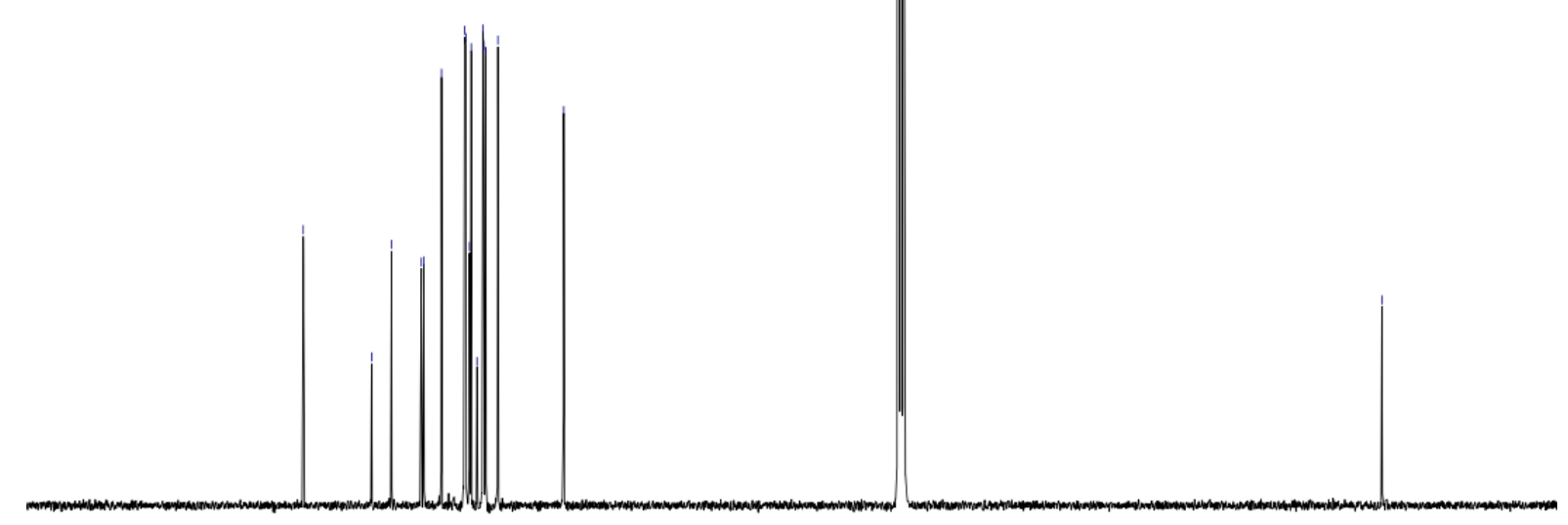

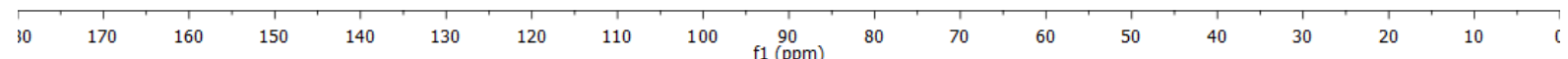




\section{$\underline{\text { 2,6-bis(1-(3,5-difluorophenyl)vinyl)-4-methylaniline 12i }}$}
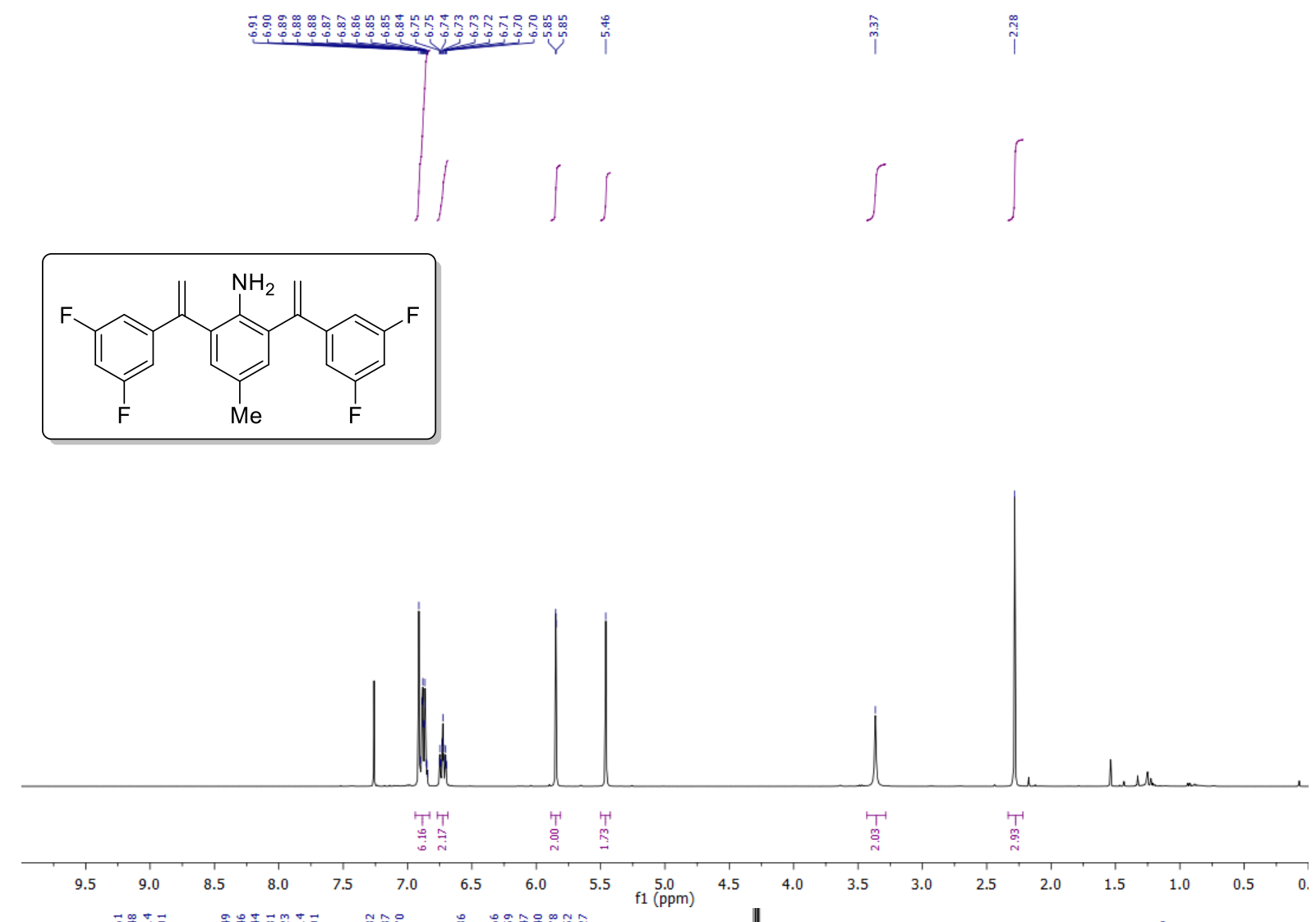

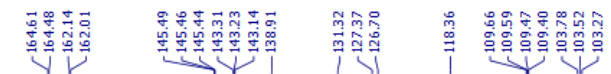

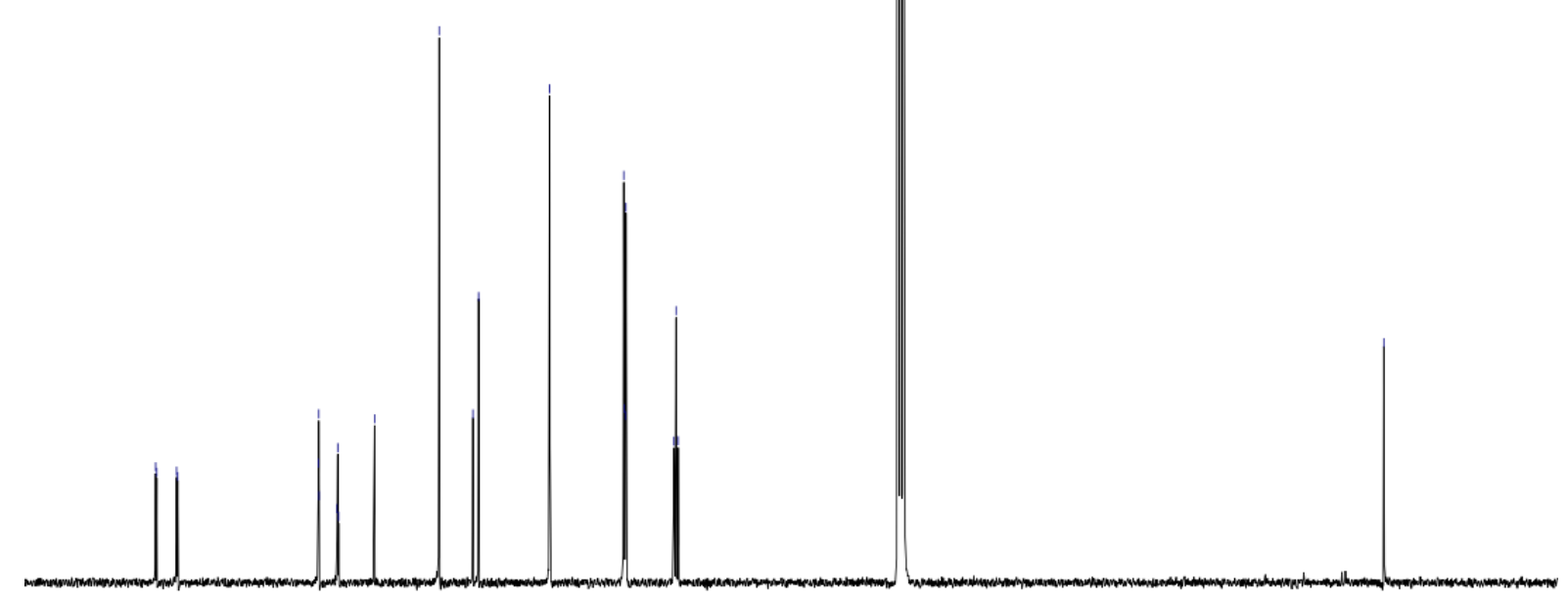


WILEY-VCH

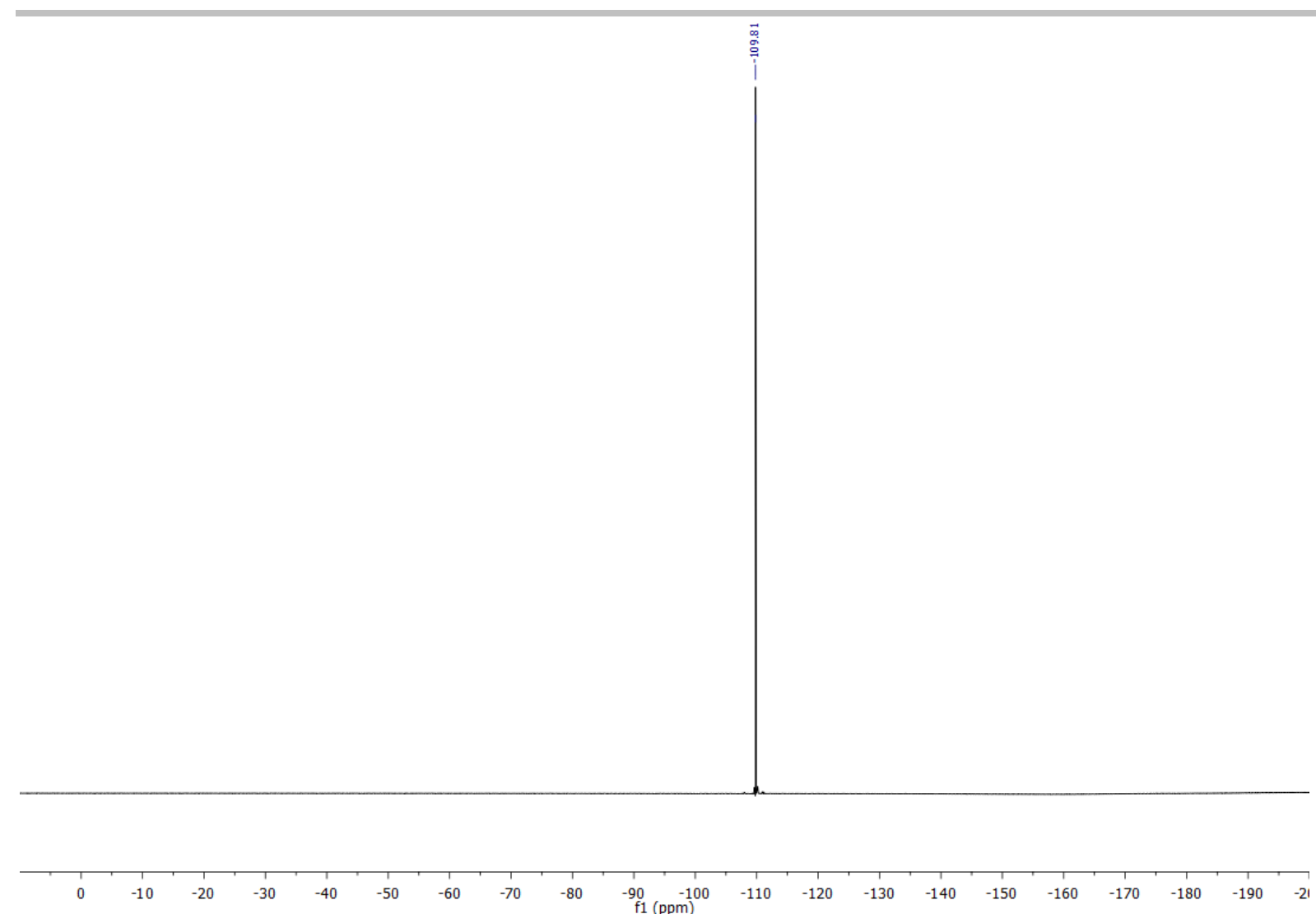




\section{2,6-bis((R)-1-phenylethyl)aniline $1 \mathrm{~b}$}

s.
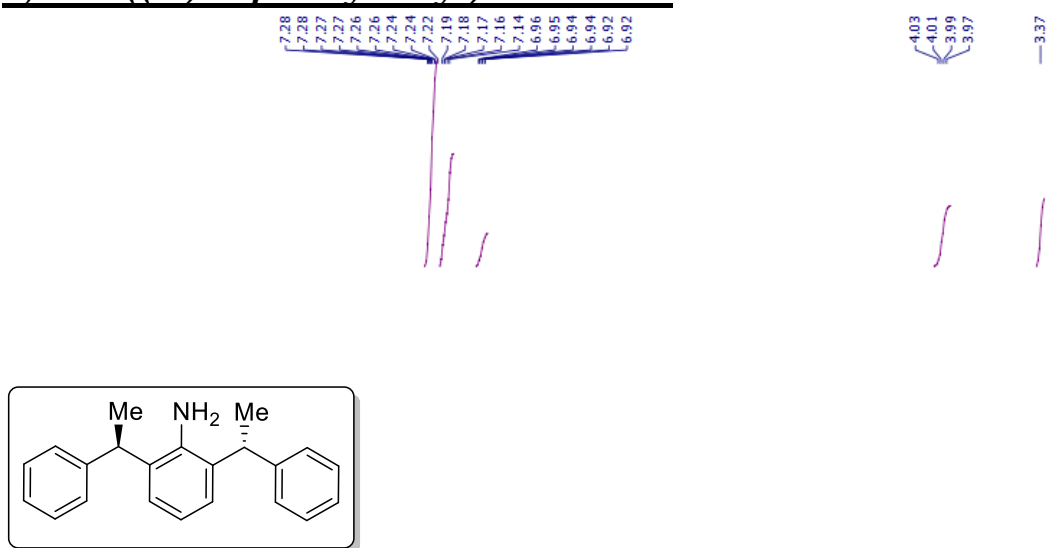

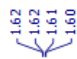
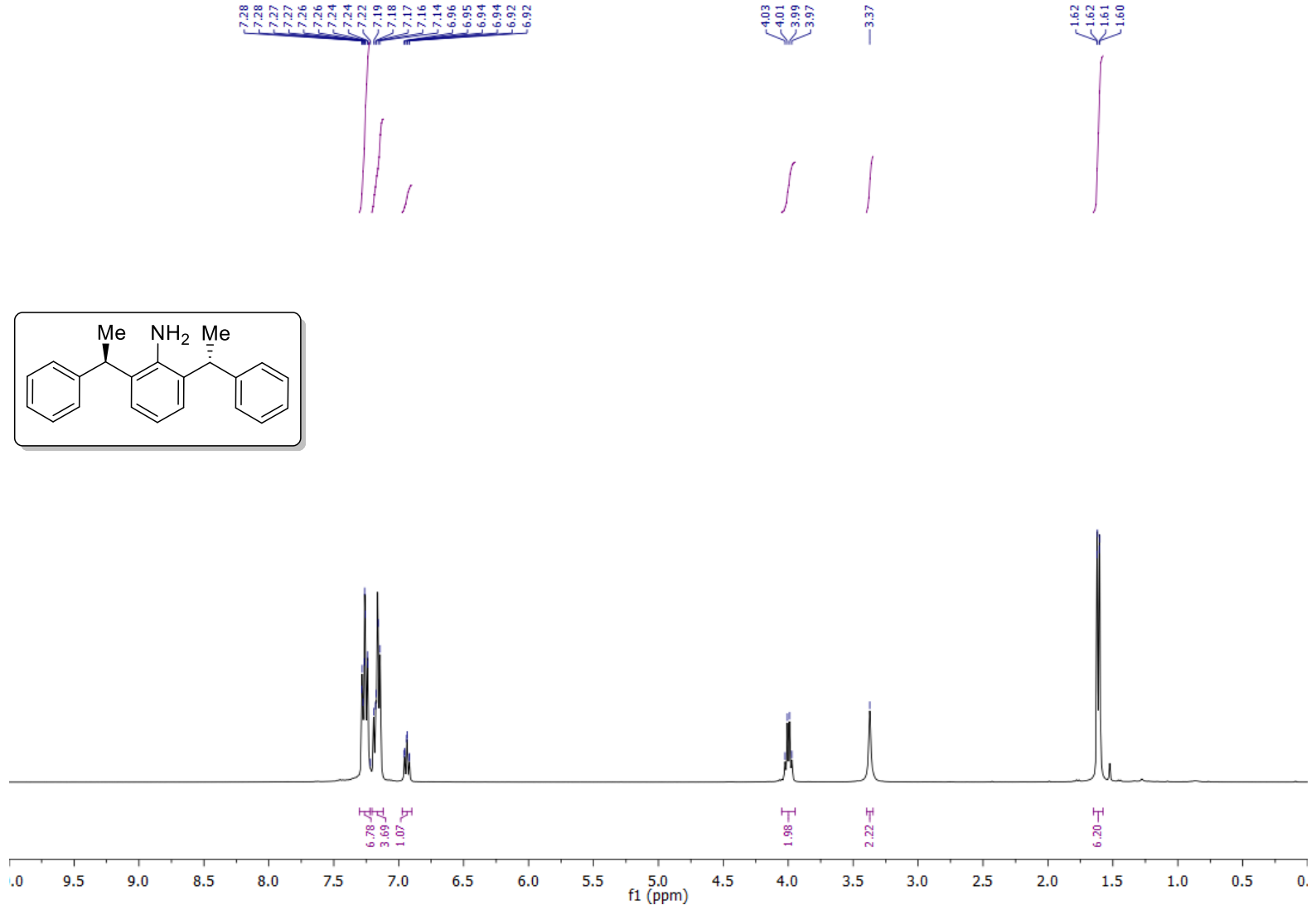

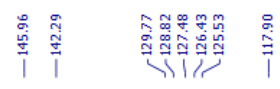
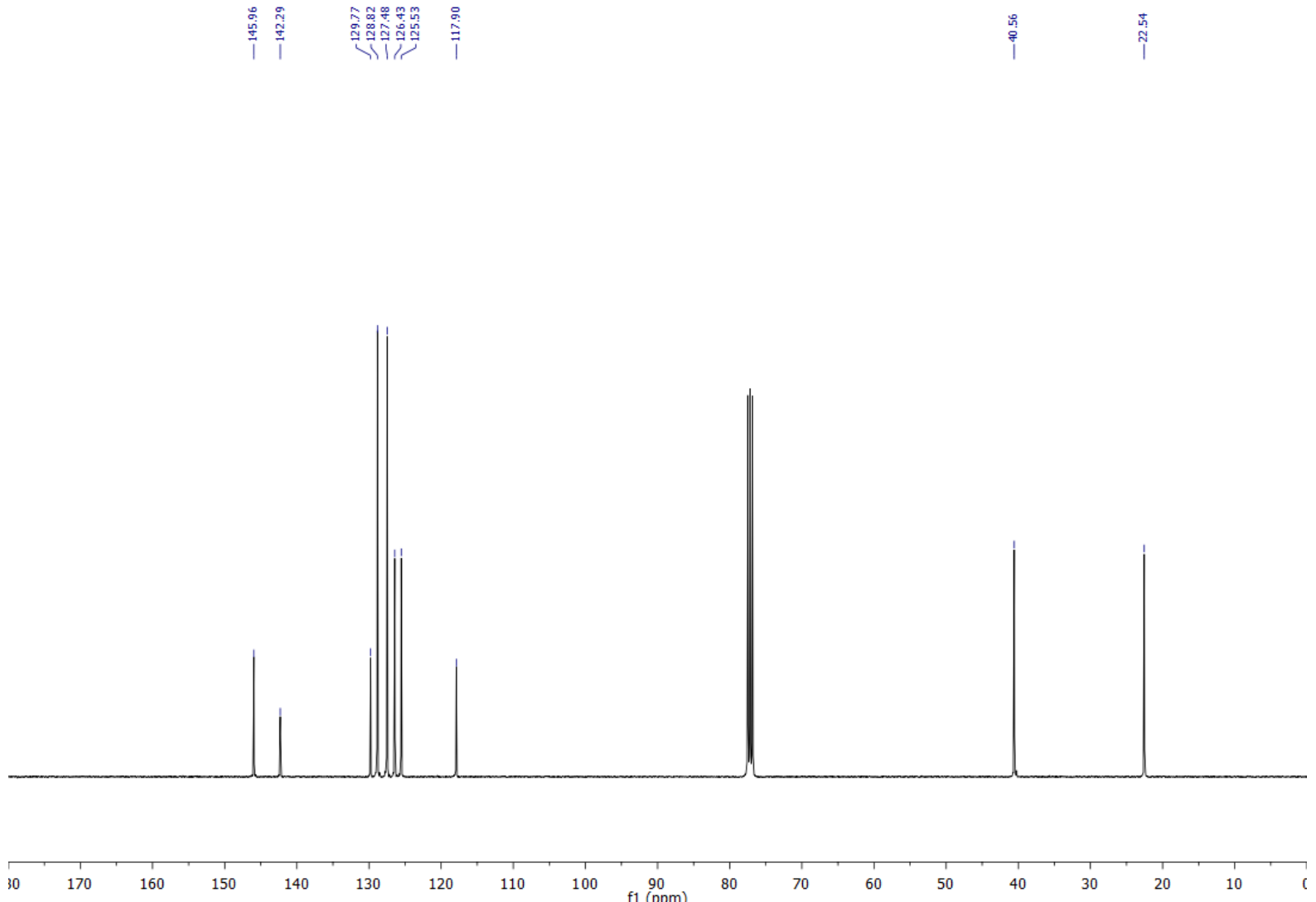
2,6-bis((R)-1-phenylethyl)-4-(trifluoromethyl)aniline 1e
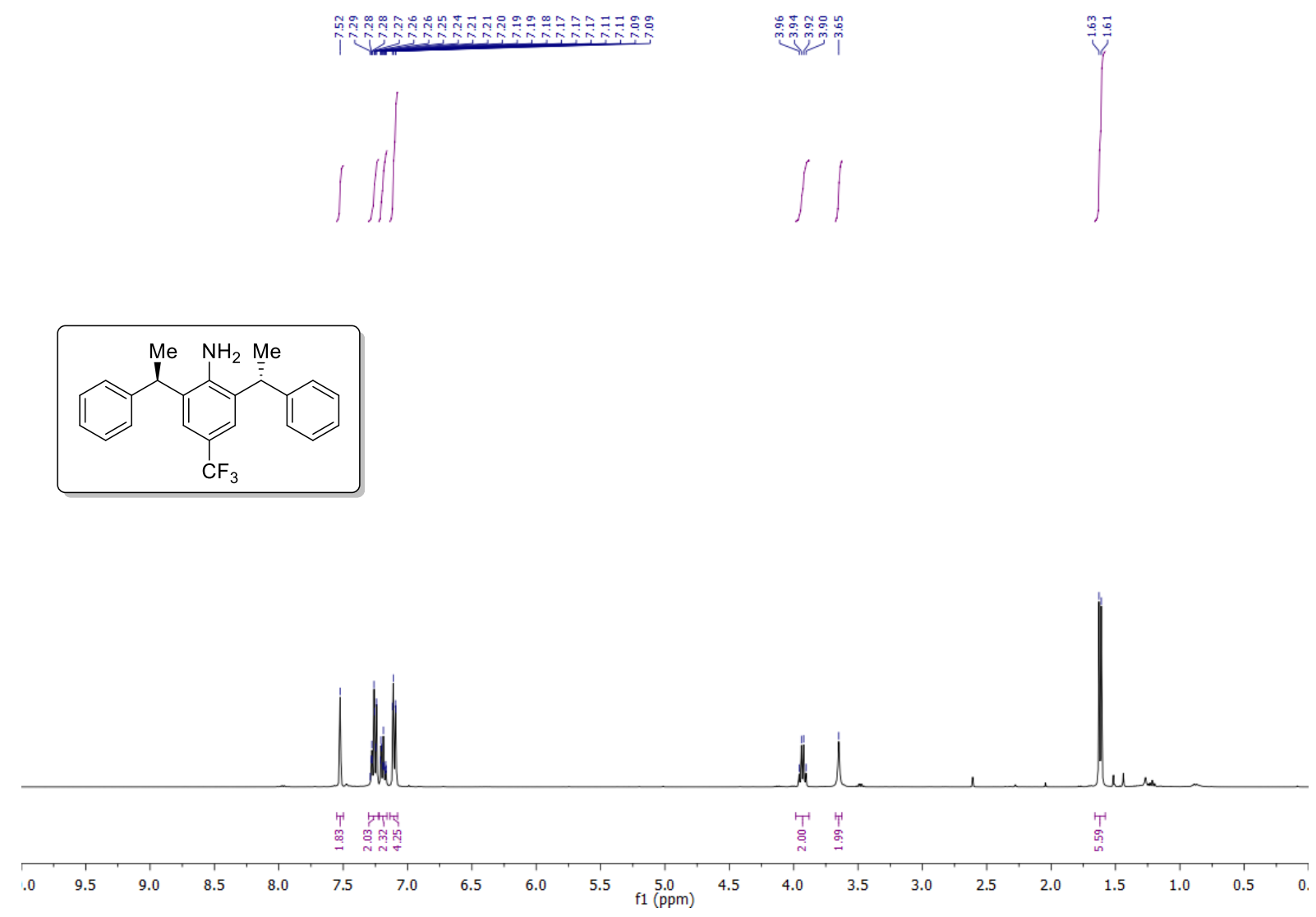

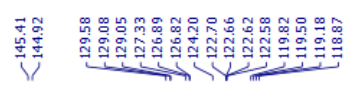

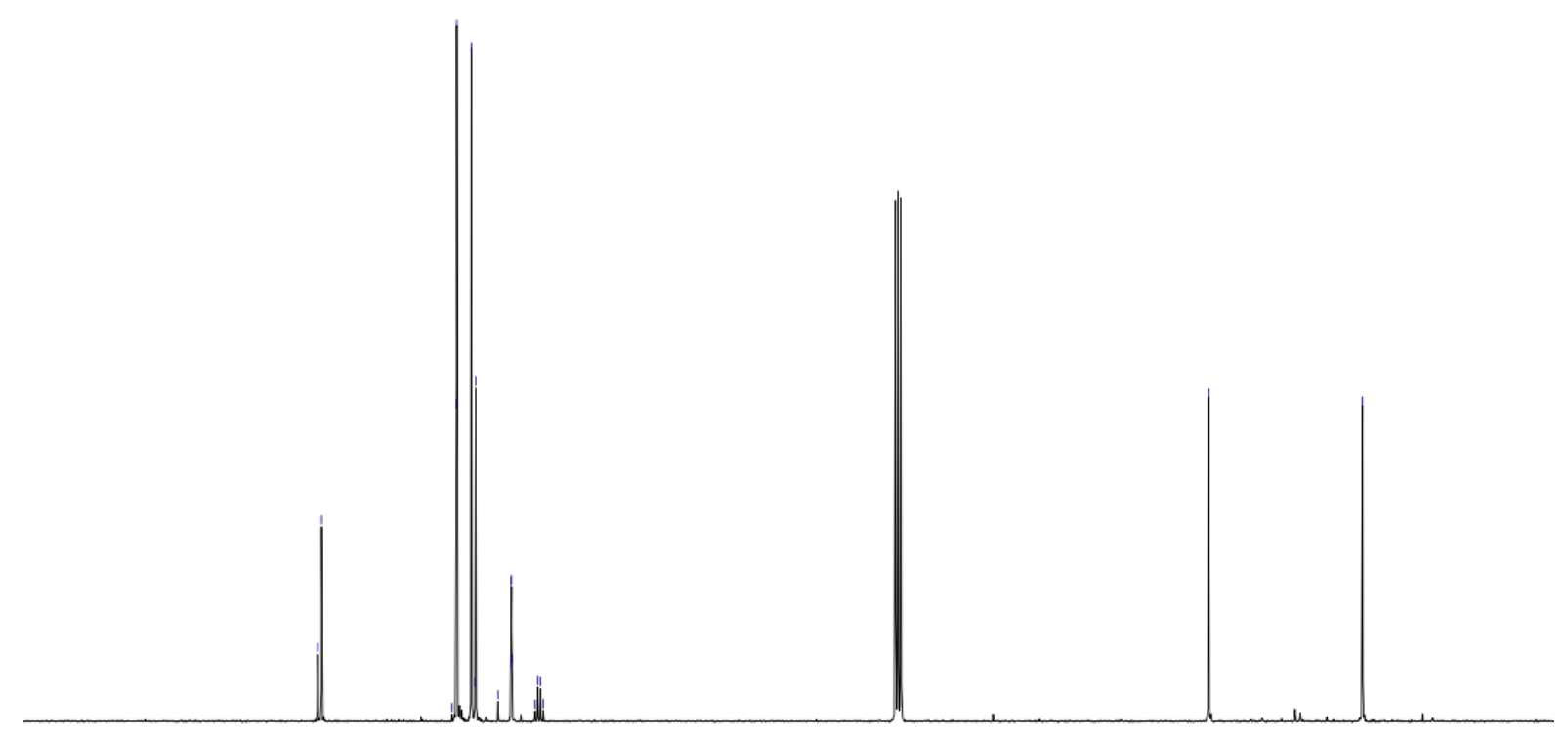

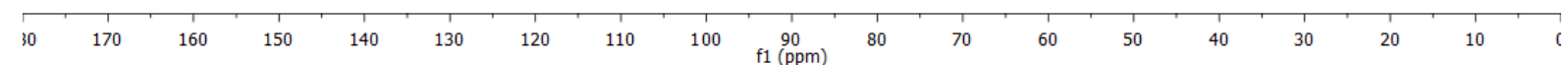




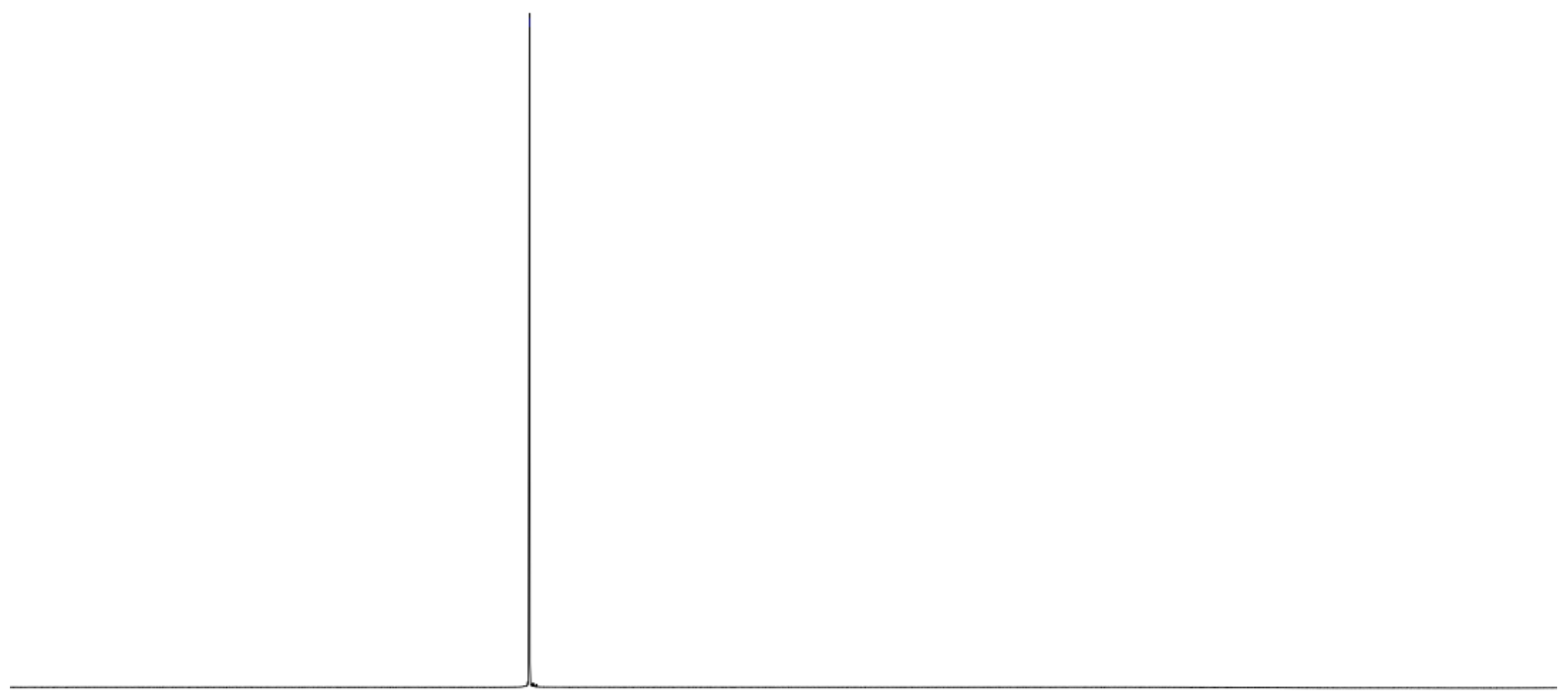

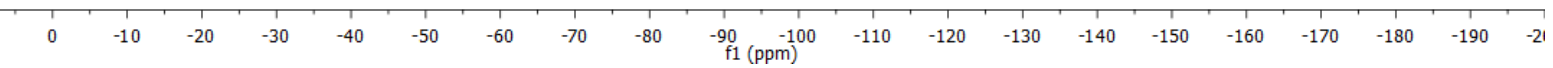




\section{$\underline{\text { 2,6-bis((R)-1-(4-(tert-butyl)phenyl)ethyl)-4-methylaniline If }}$}
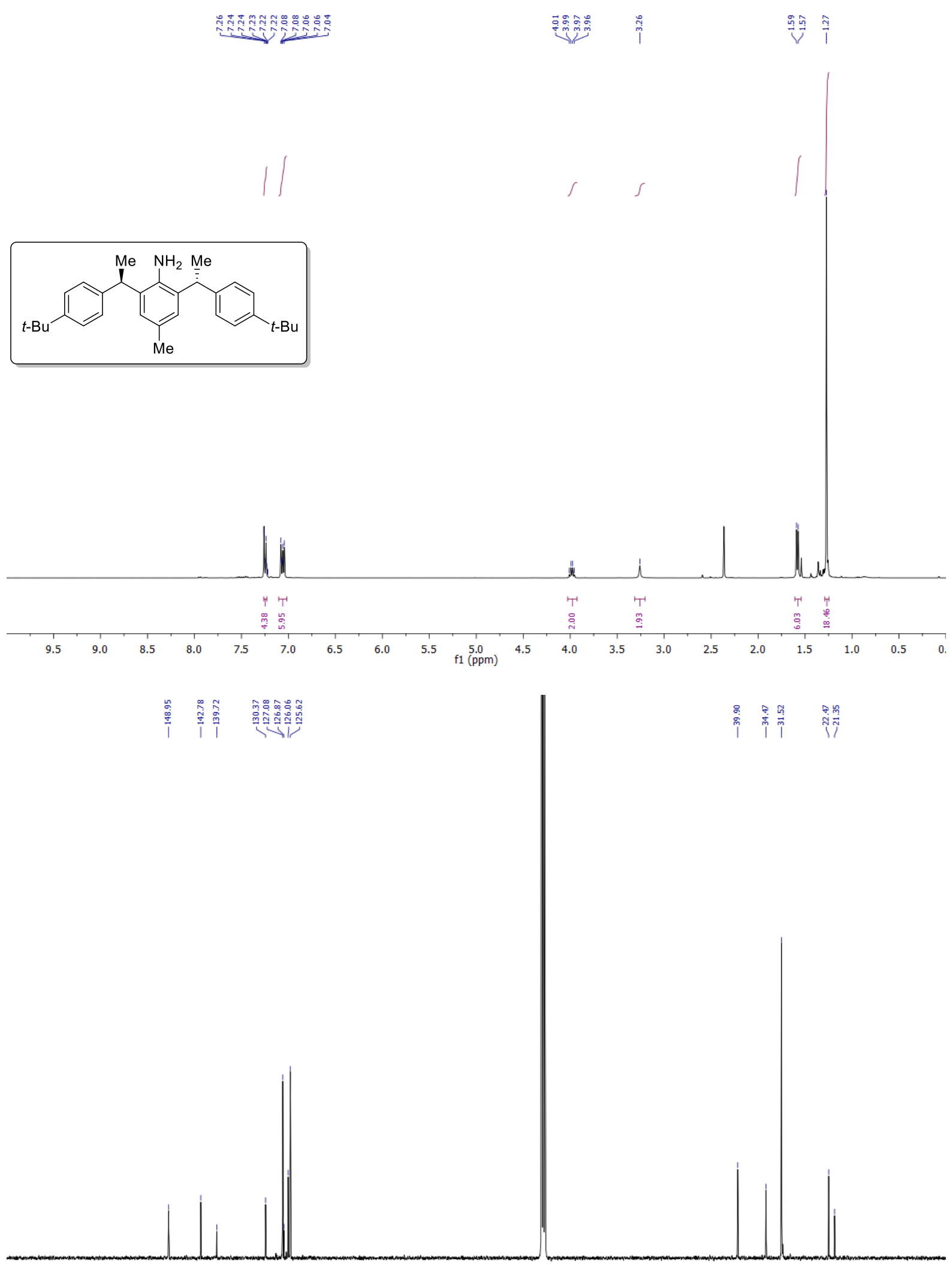

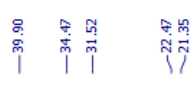




\section{4-methyl-2,6-bis((R)-1-(naphthalen-2-yl)ethyl)aniline 1g}
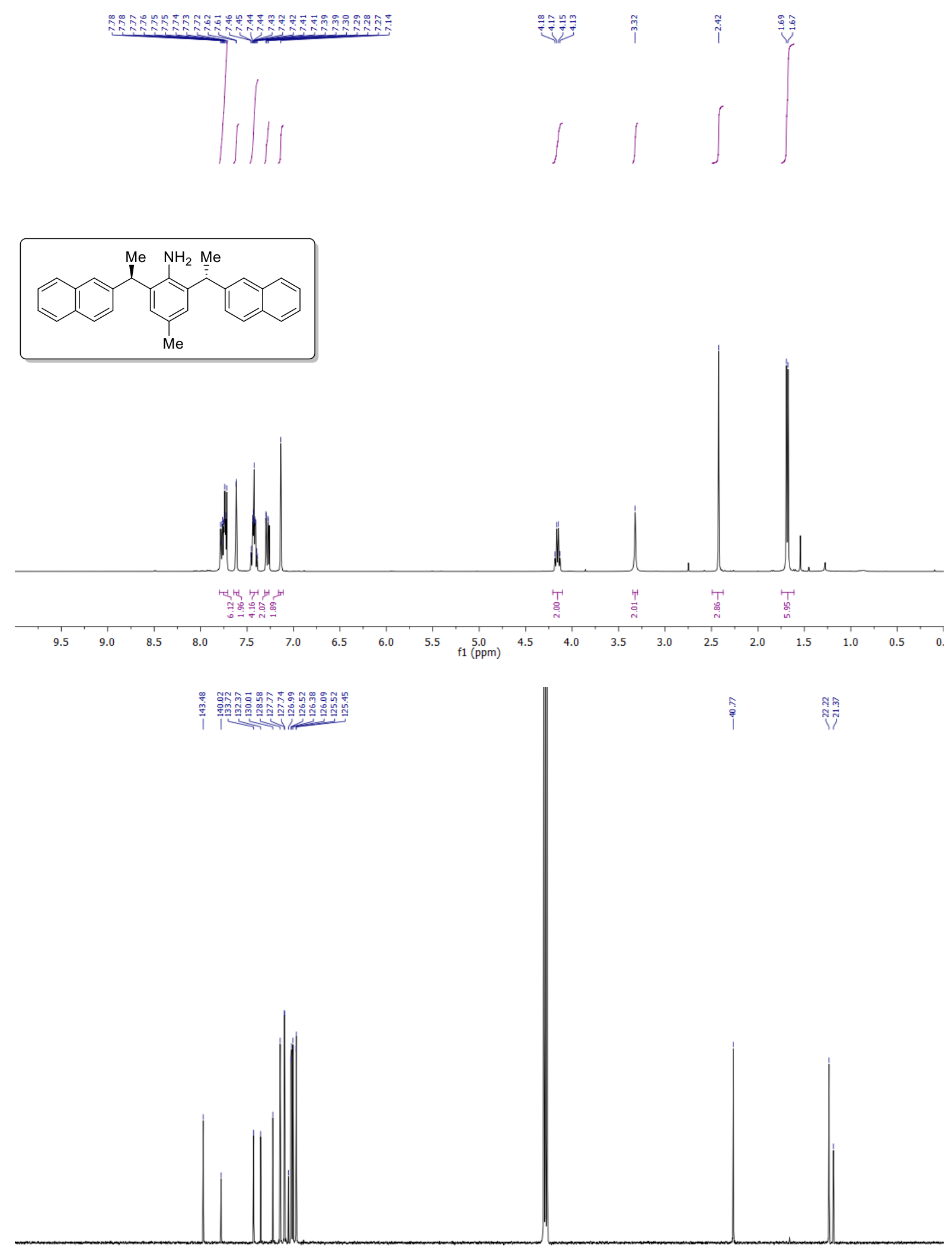


\section{$\underline{\text { 2,6-bis }((R)-1-(3,5-d i f l u o r o p h e n y l) e t h y l)-4-m e t h y l a n i l i n e ~ 1 i}$}
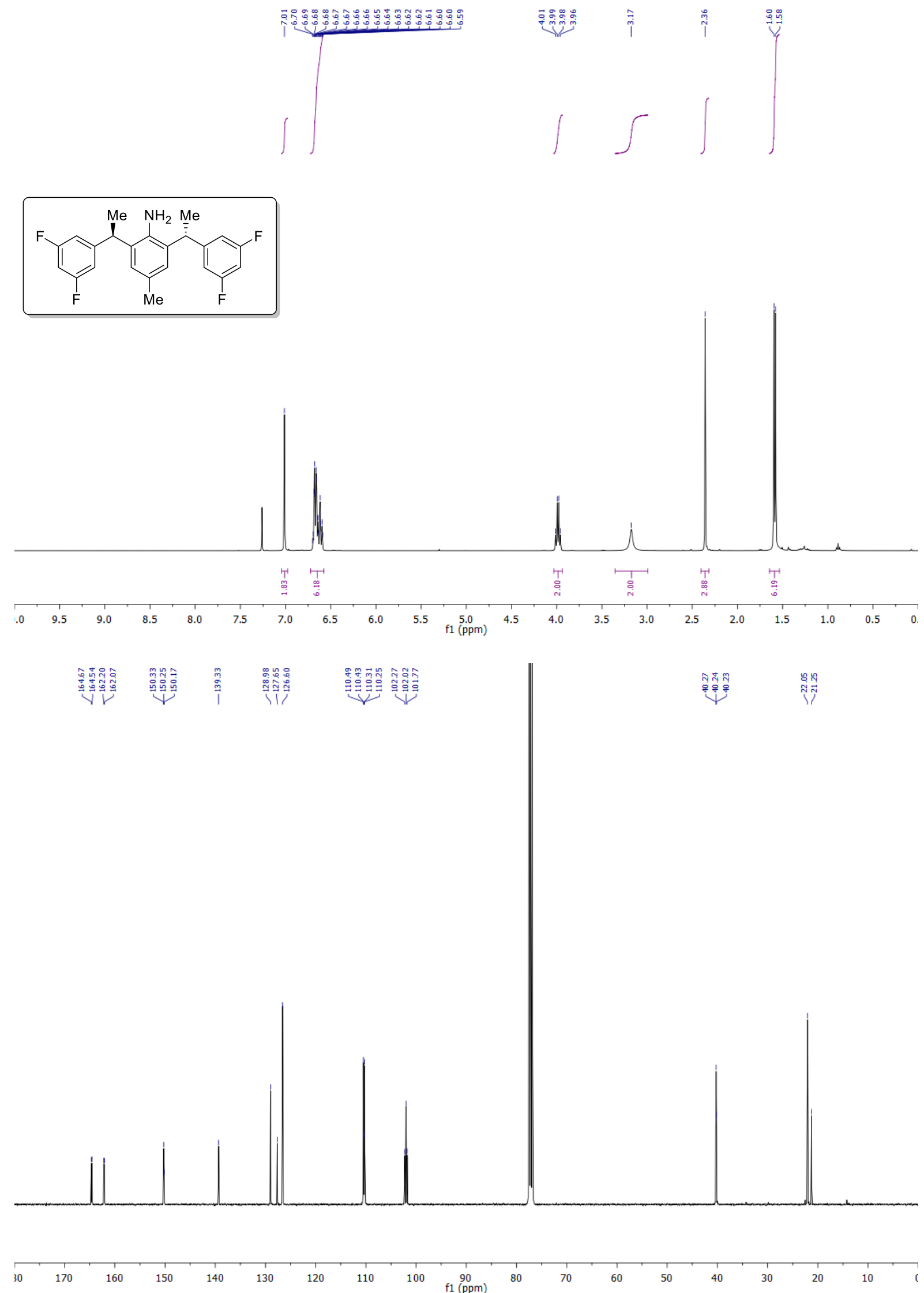
WILEY-VCH

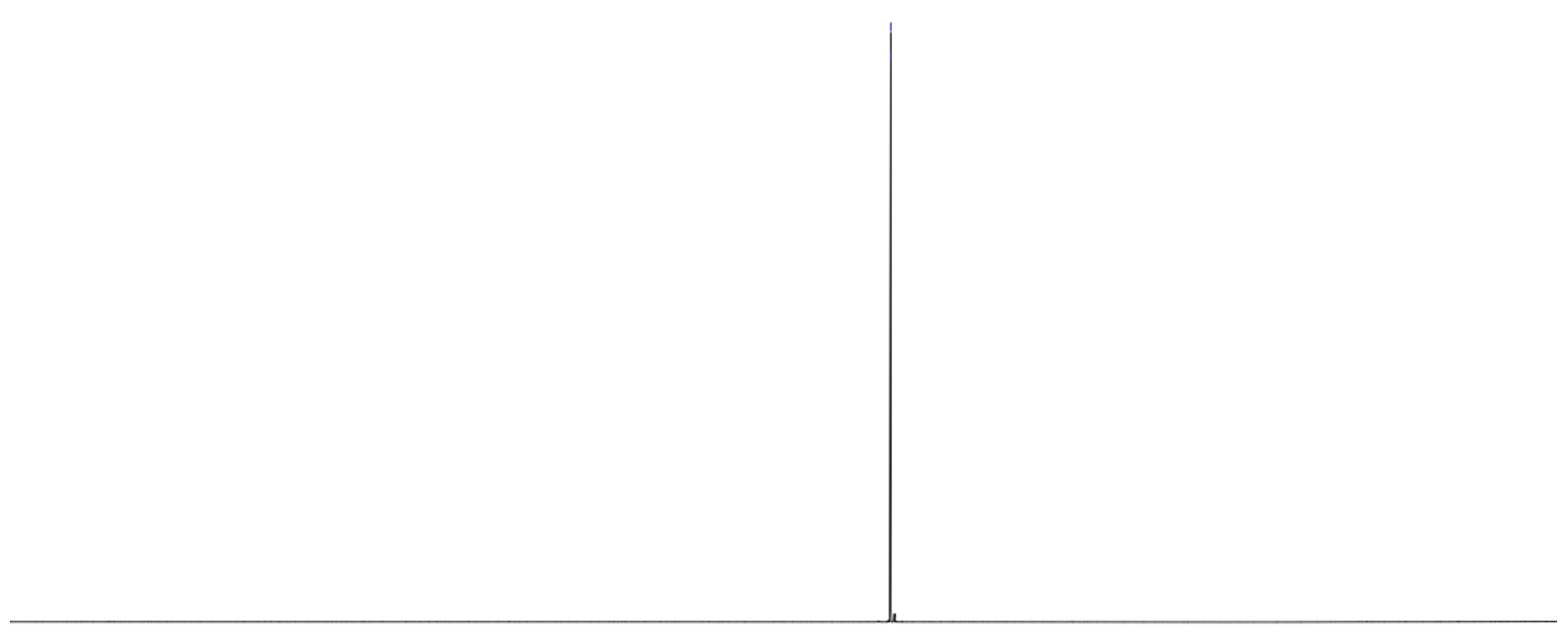

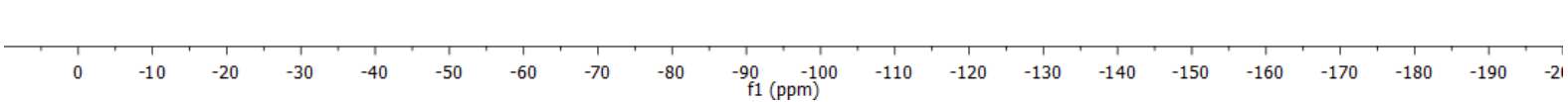




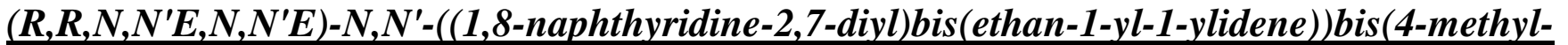
2,6-bis ((R)-1-phenylethyl)aniline) L1

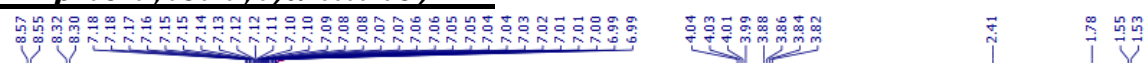

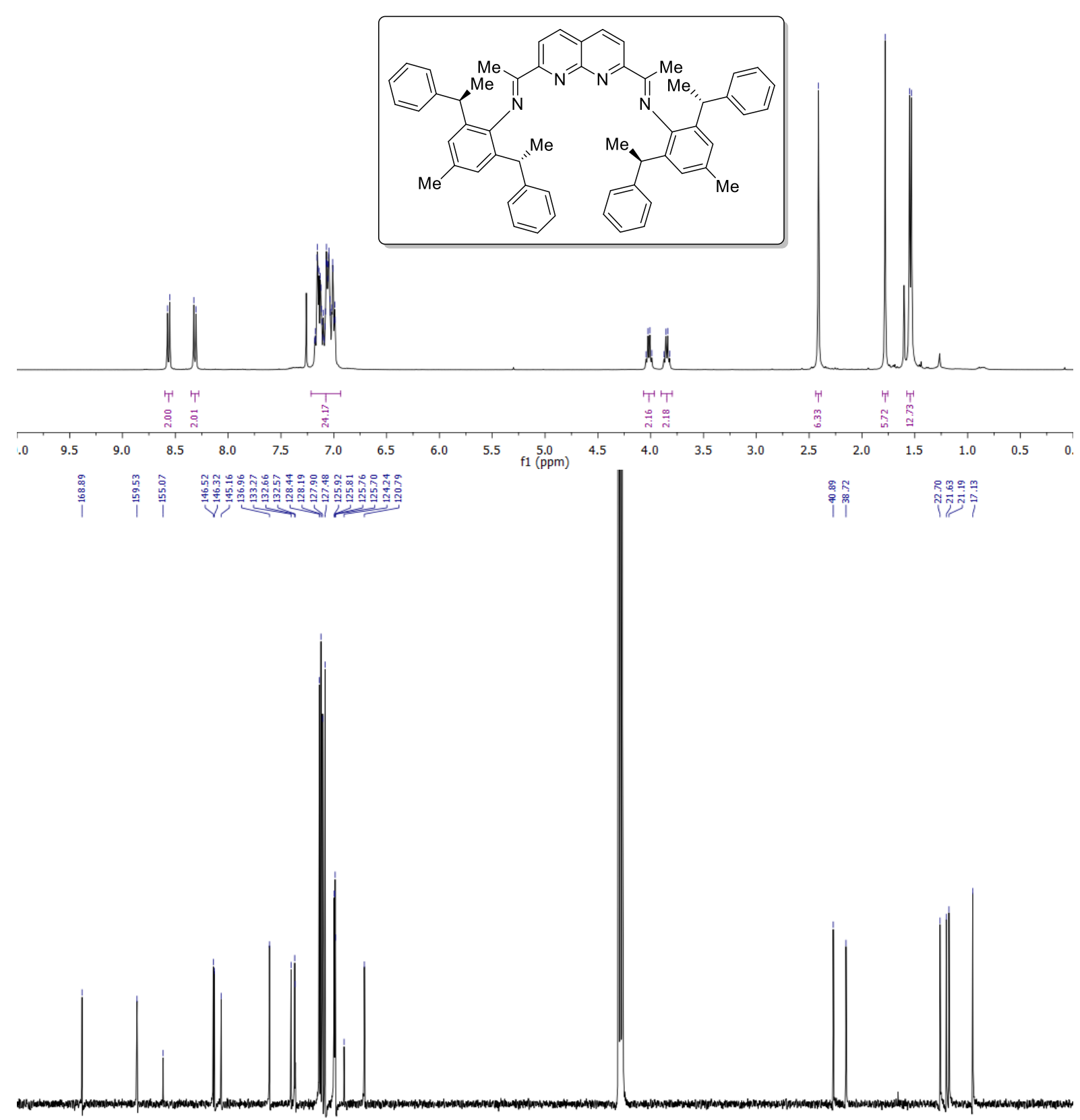

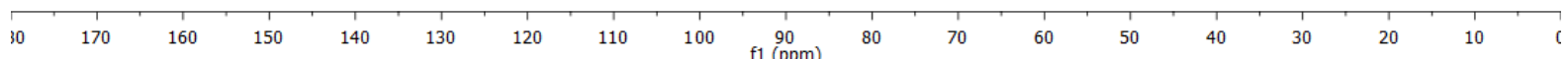


$\underline{\left(R, R, N, N^{\prime} E, N, N^{\prime} E\right)-N, N^{\prime}-((1,8-\text {-naphthyridine-2,7-divl)bis(ethan-1-yl-1-ylidene))bis(2,6- }}$ bis((R)-1-phenylethyl)aniline) L2

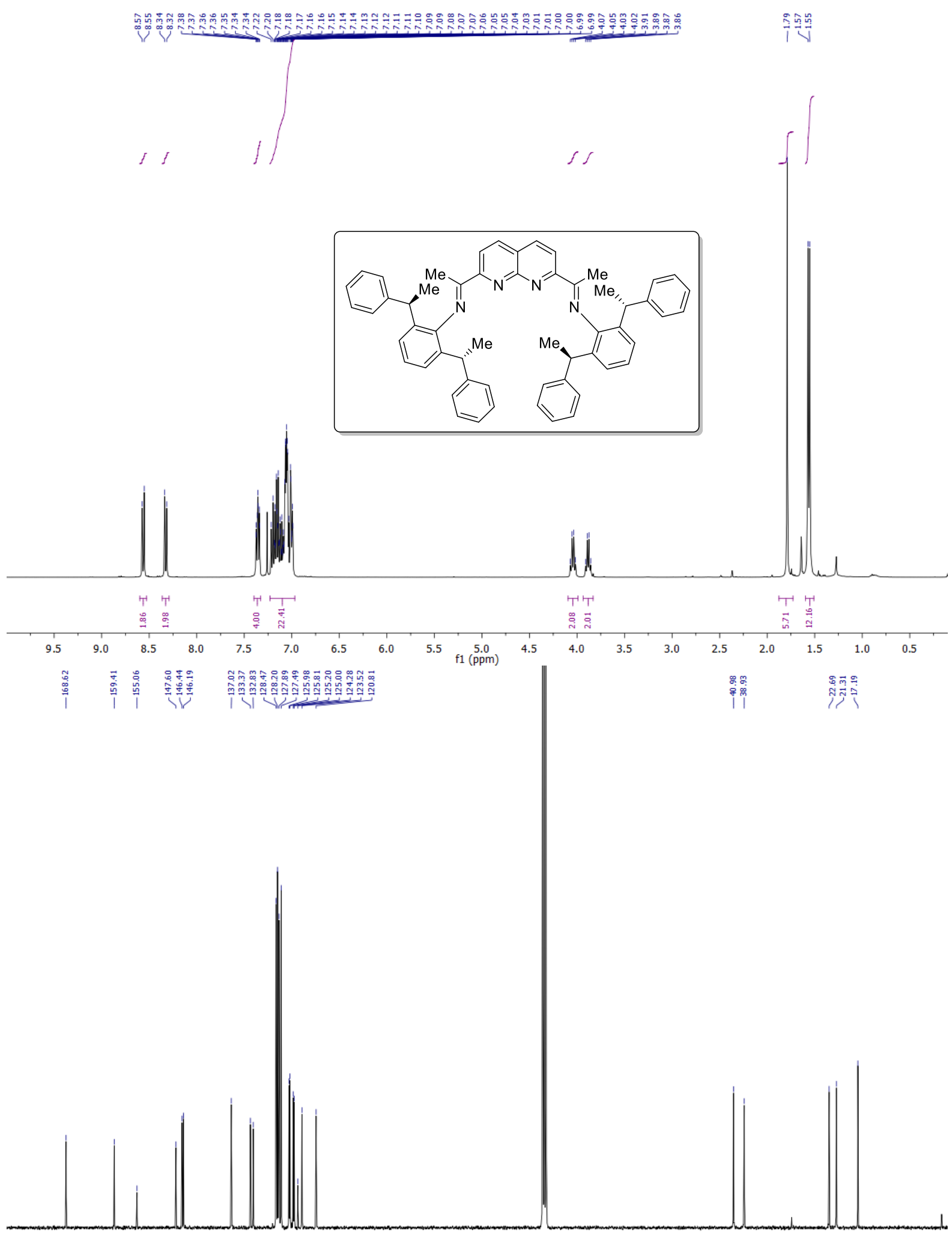

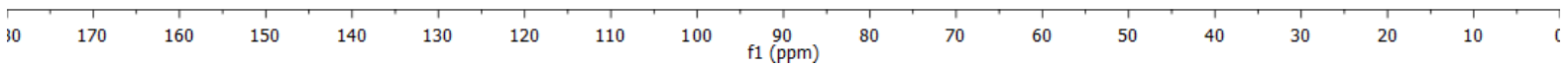


$\underline{\left(R, R, N, N^{\prime} E, N, N^{\prime} E\right)-N, N^{\prime}-((1,8-\text {-naphthyridine-2,7-diyl)bis(ethan-1-yl-1-ylidene))bis(4- }}$ methoxy-2,6-bis((R)-1-phenylethyl)aniline) L3
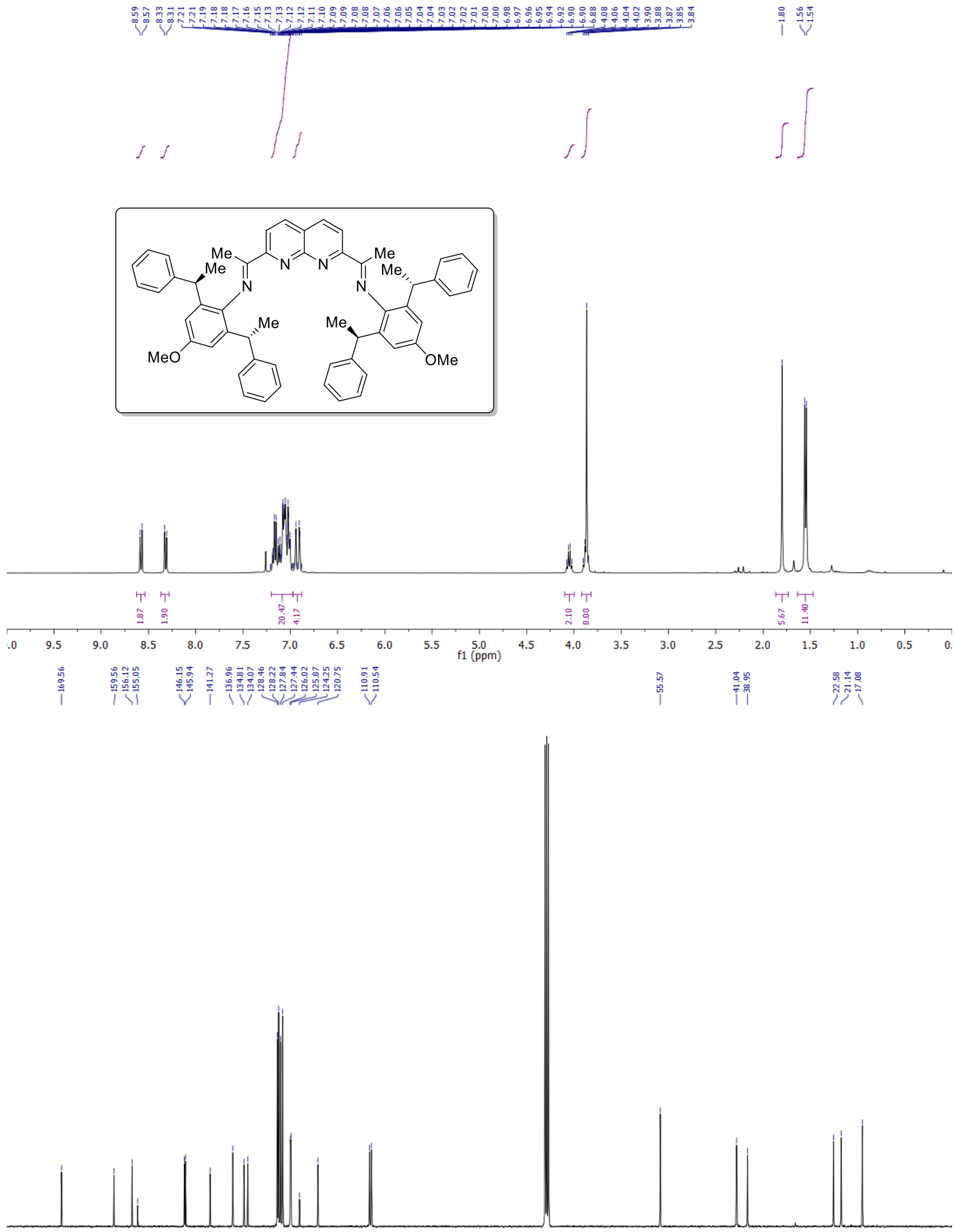

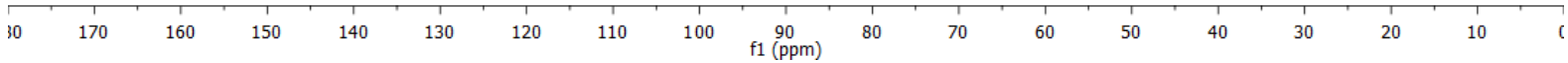




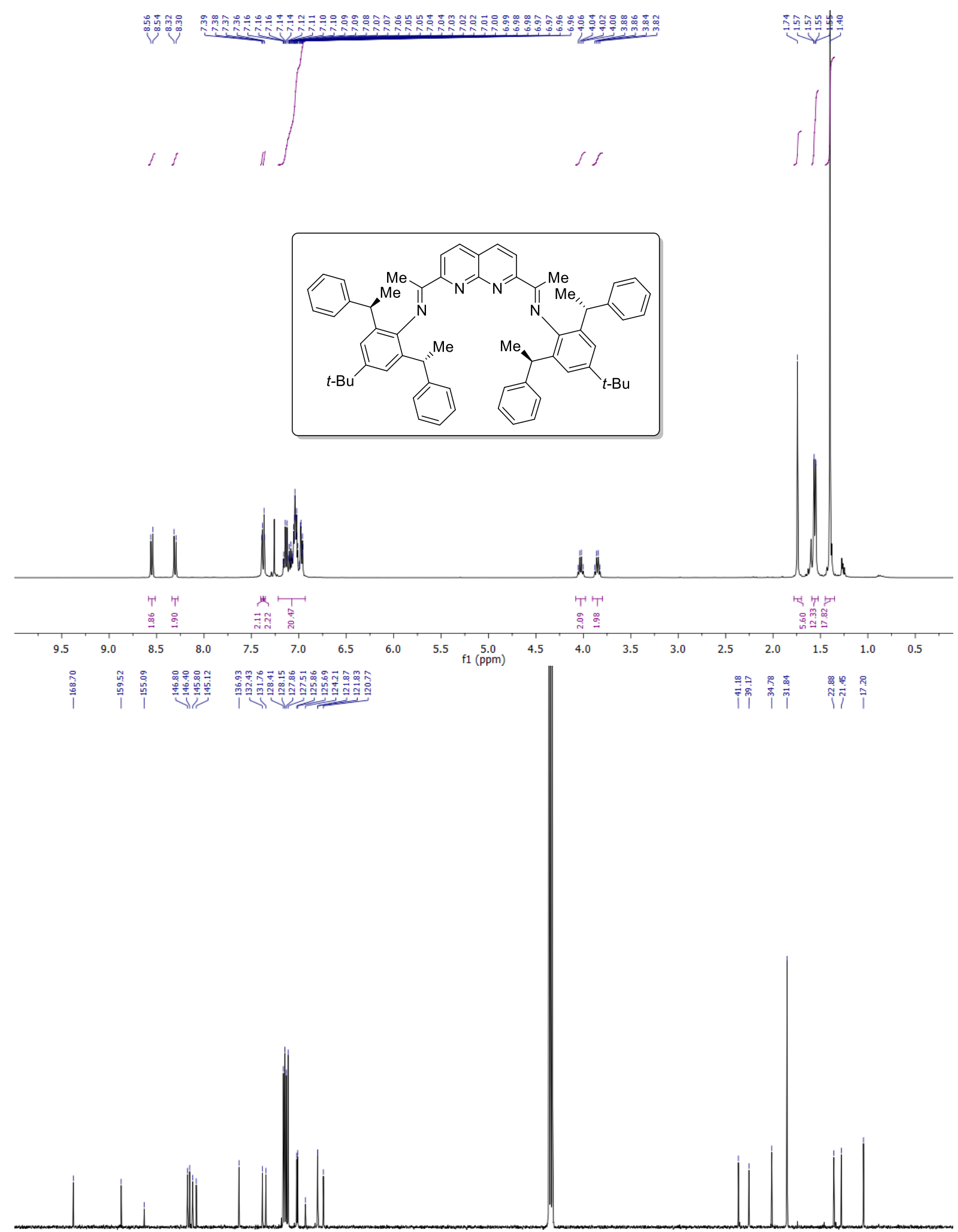

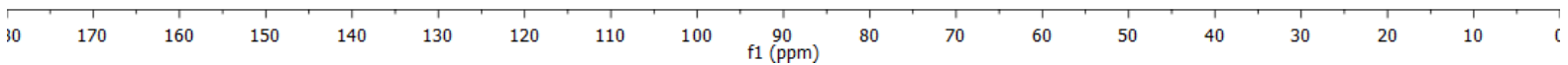



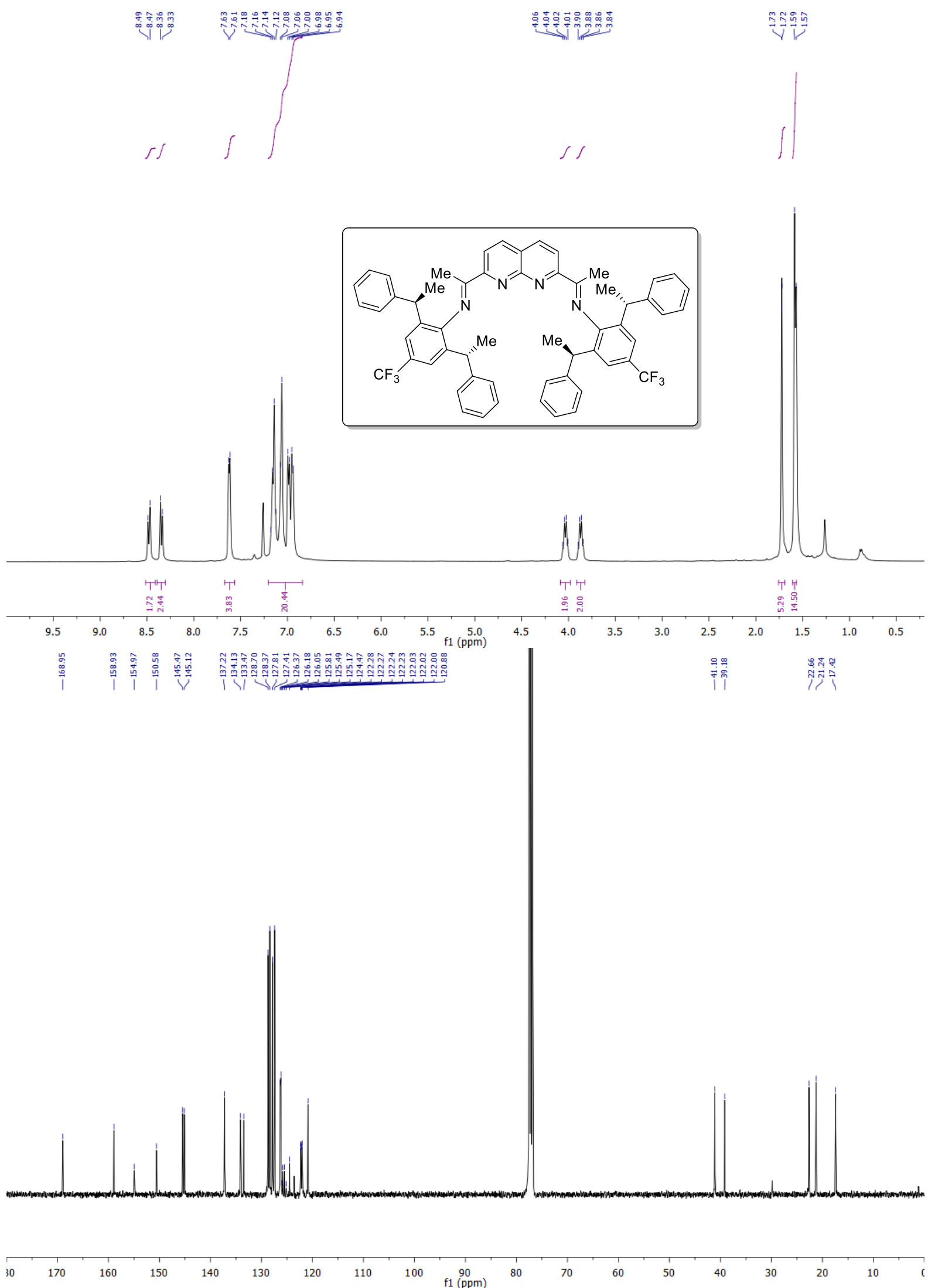
WILEY-VCH

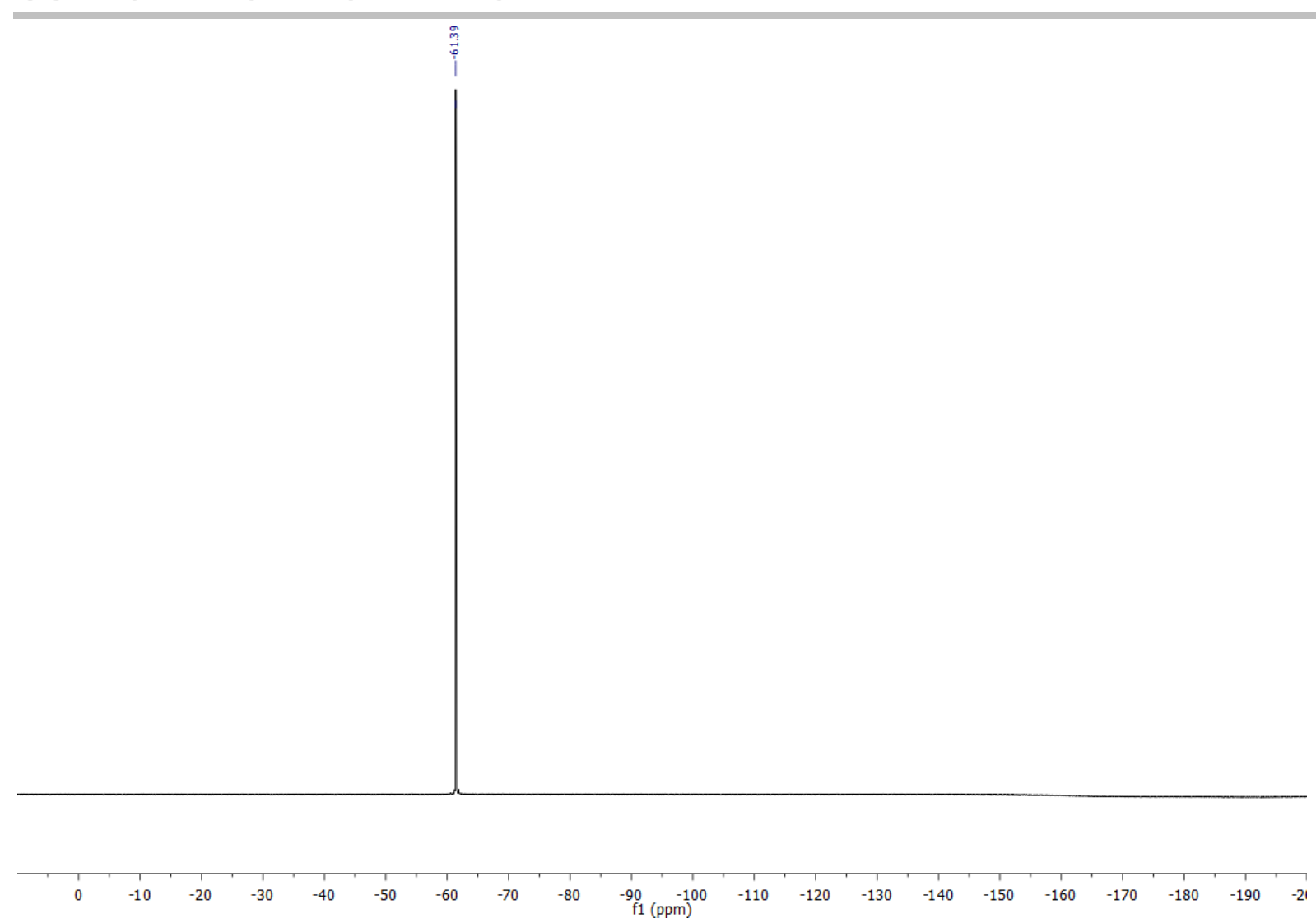


(E)-N-(1-(7-((E)-1-((2,6-bis((R)-1-(4-(tert-butyl)phenyl)ethyl)-4-methylphenyl)imino)ethyl)-1,8naphthyridin-2-yl)ethylidene)-2-((R)-1-(3-(tert-butyl)phenyl)ethyl)-6-((R)-1-(4-(tertbutyl)phenyl)ethyl)-4-methylaniline L6

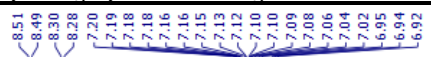

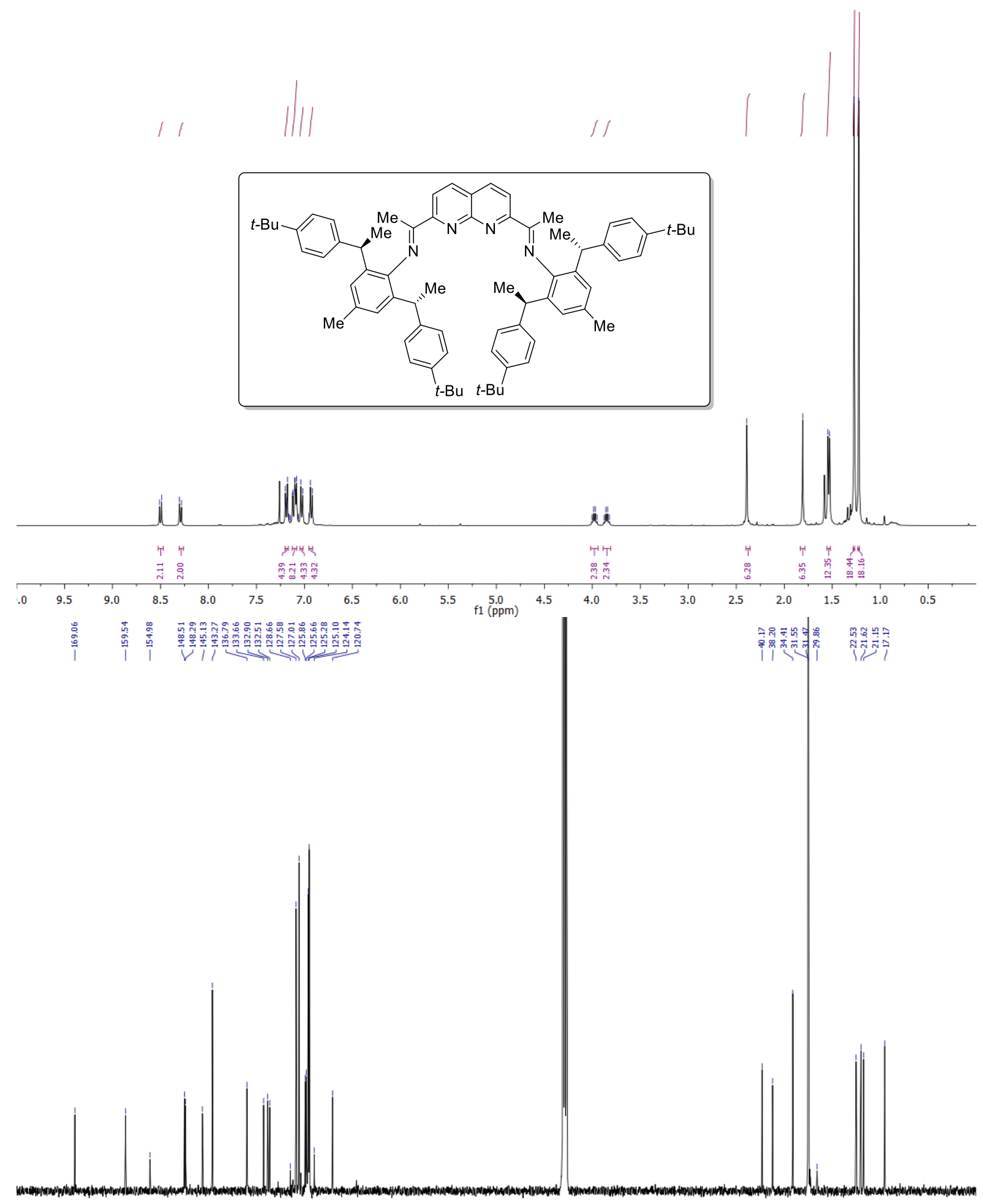




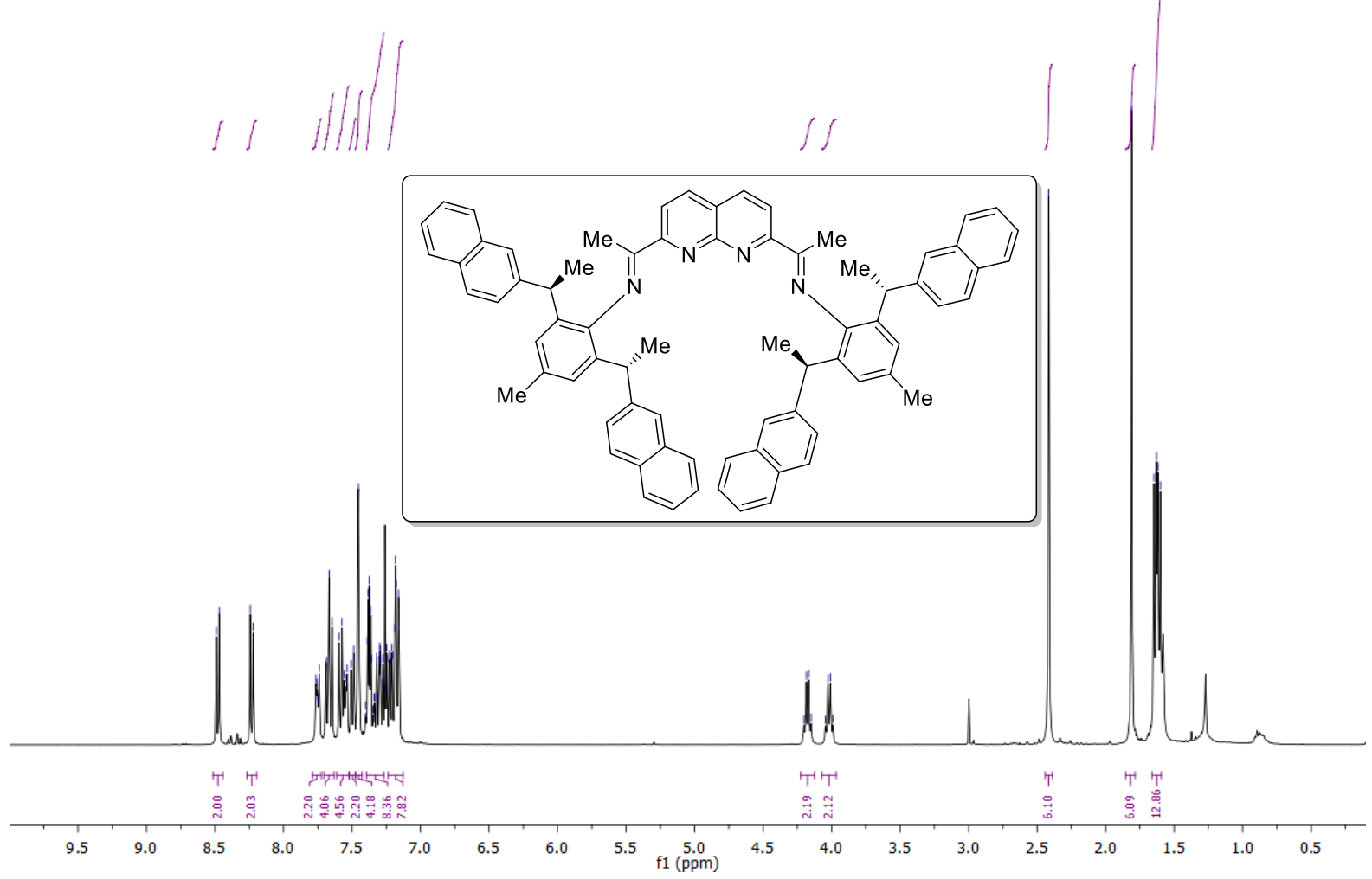

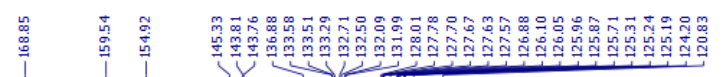

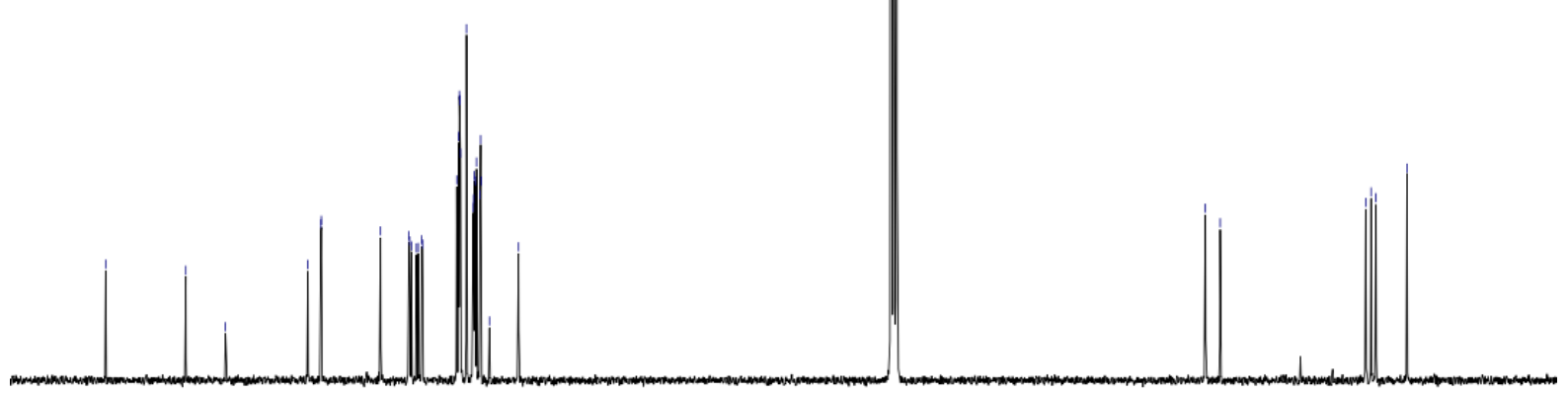


$\underline{\left(R, R, N, N^{\prime} E, N, N^{\prime} E\right)-N, N^{\prime}-((1,8-\text {-naphthyridine-2,7-divl)bis(ethan-1-yl-1-ylidene))bis(2,6- }}$ bis((R)-1-(3,5-dimethylphenyl)ethyl)-4-methylaniline $) L 8$
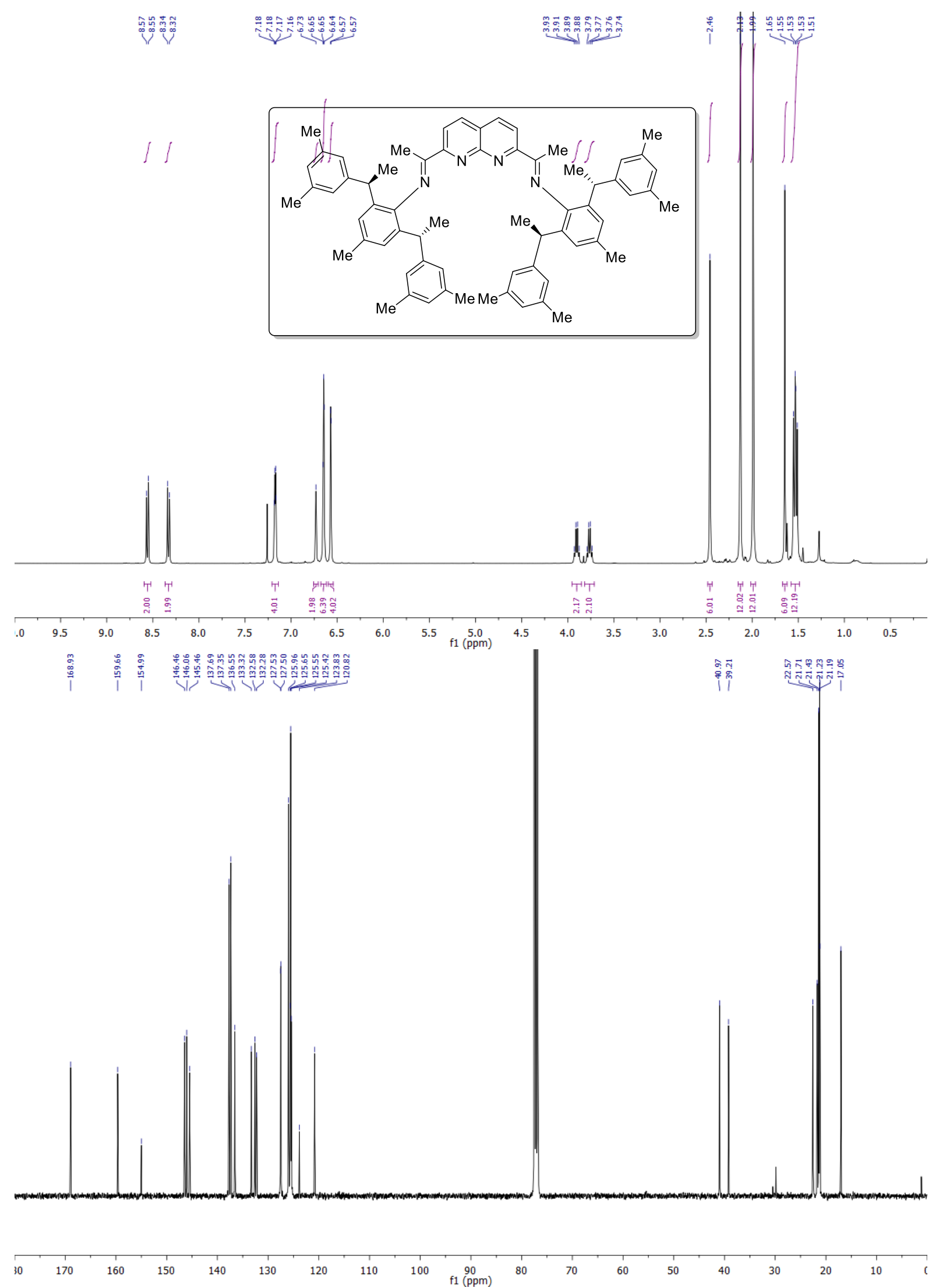


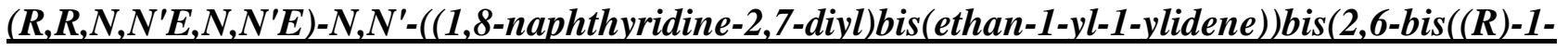

(3,5-difluorophenyl)ethyl)-4-methylaniline) L9

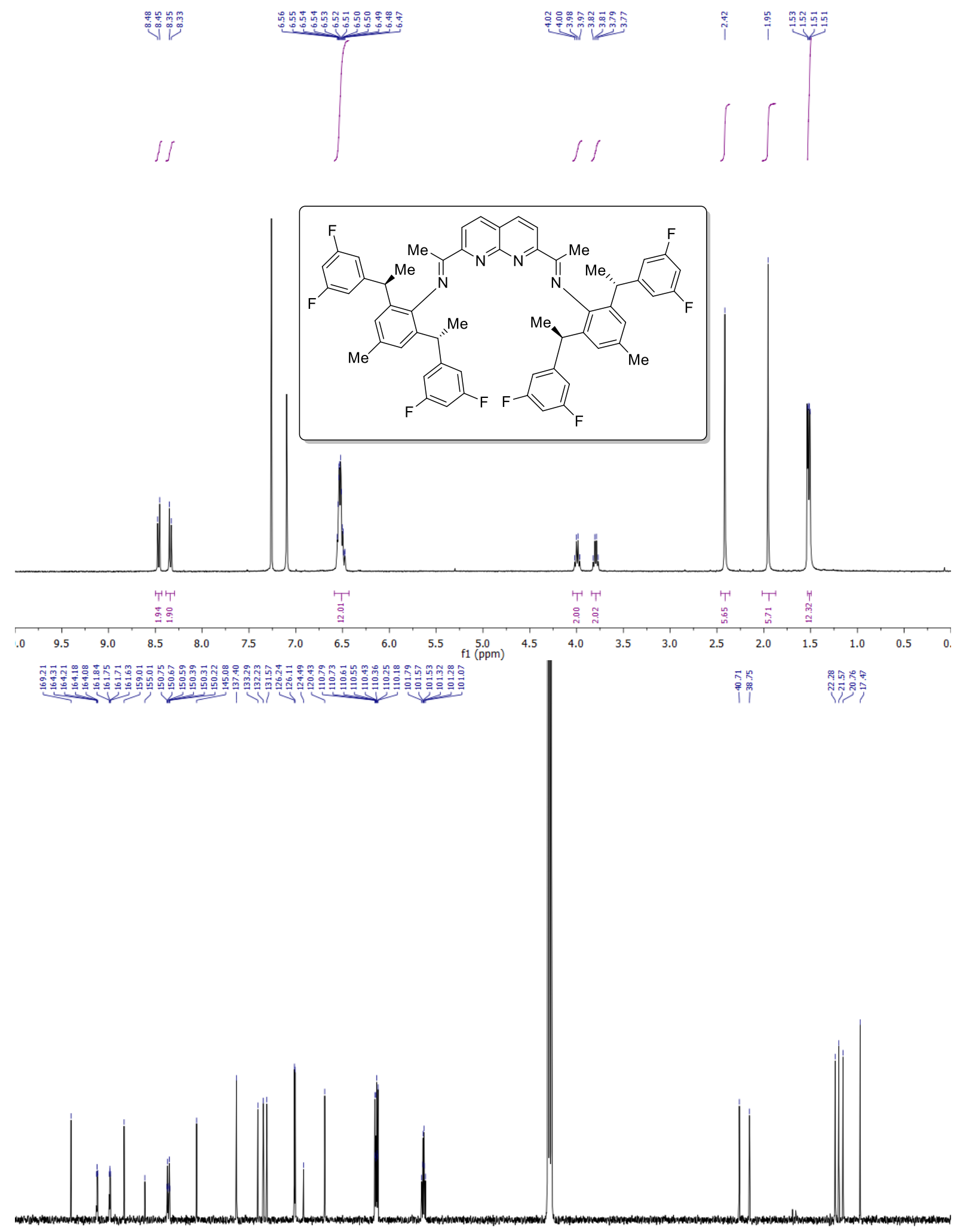

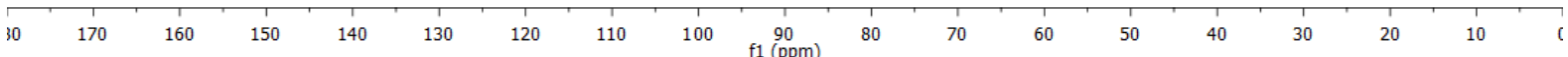


WILEY-VCH

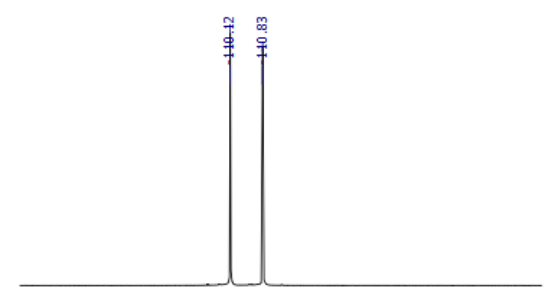

$-106-107-108-109-110-111-112-113-114-115-116$

$1(\mathrm{ppm})$

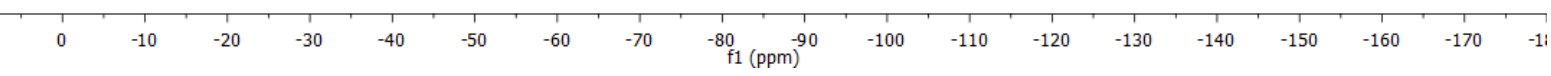




\section{4-(2,2-dichlorovinyl)phenoxy)triisopropylsilane 14}
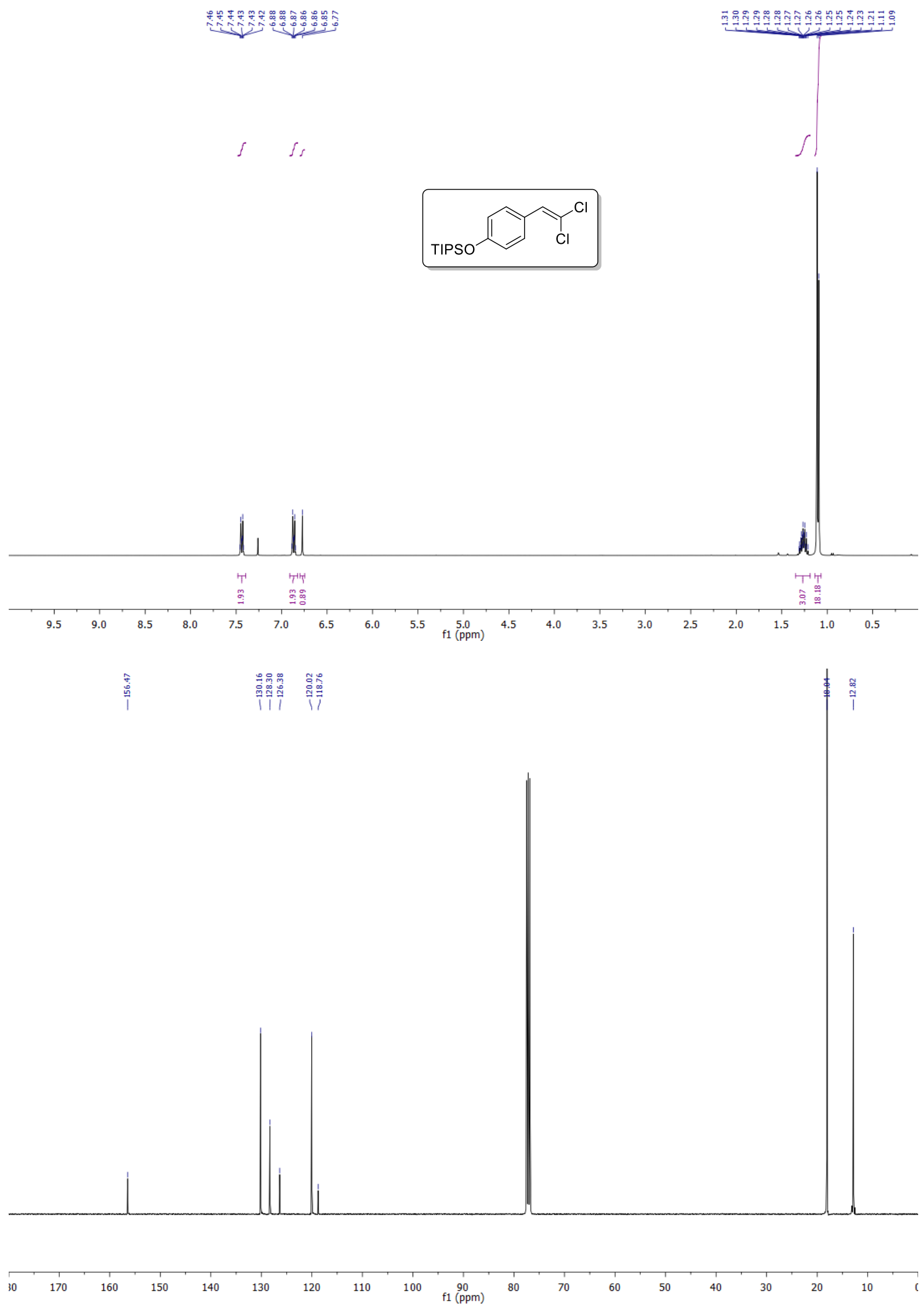


\section{$\underline{(R)-1-m e t h o x y-4-((2-p h e n y l c y c l o p r o p y l i d e n e) m e t h y l) b e n z e n e ~ 6 a a ~}$}
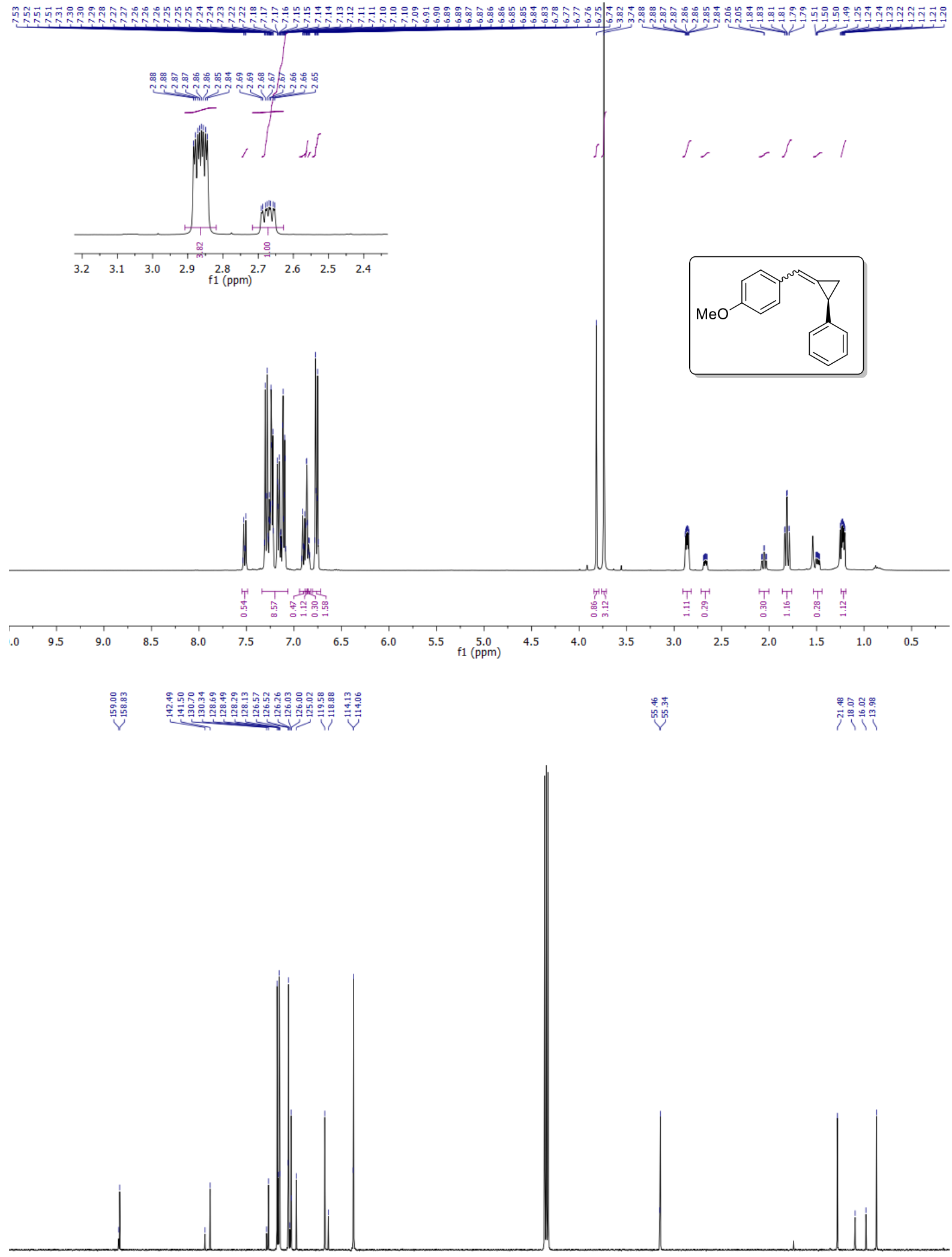

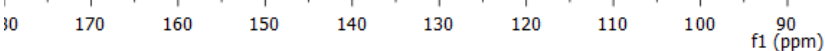




\section{(R)-1-methyl-4-((2-phenylcyclopropylidene)methyl)benzene 6ba}

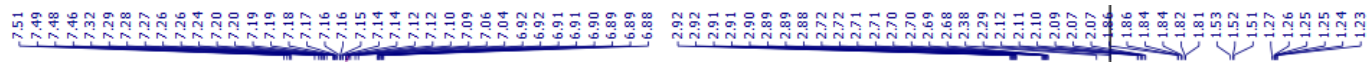
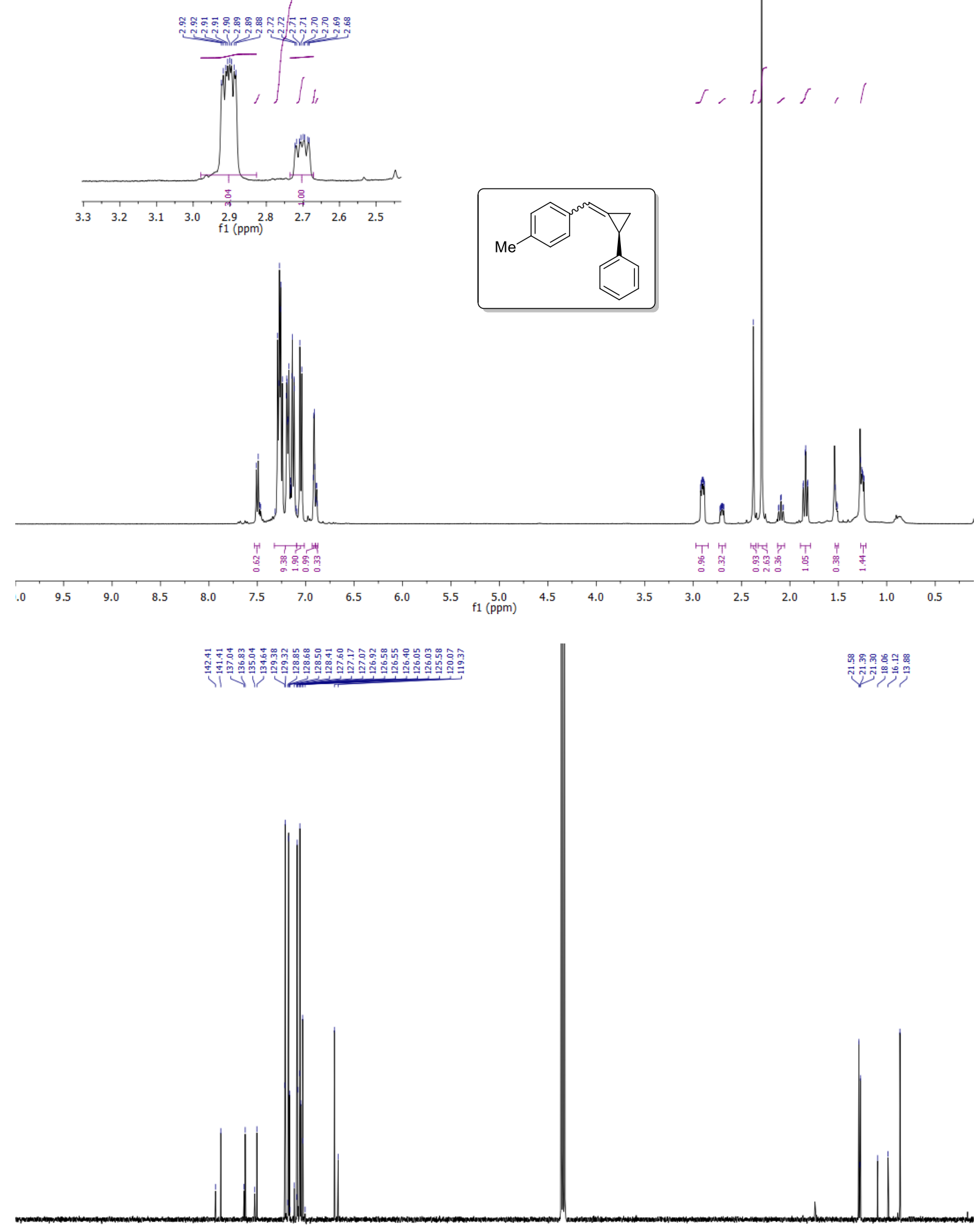

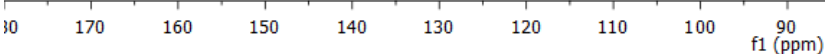




\section{(R)-triisopropyl(4-((2-phenylcyclopropylidene)methyl)phenoxy)silane 6ca}

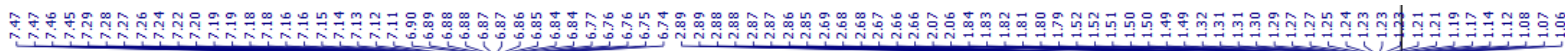
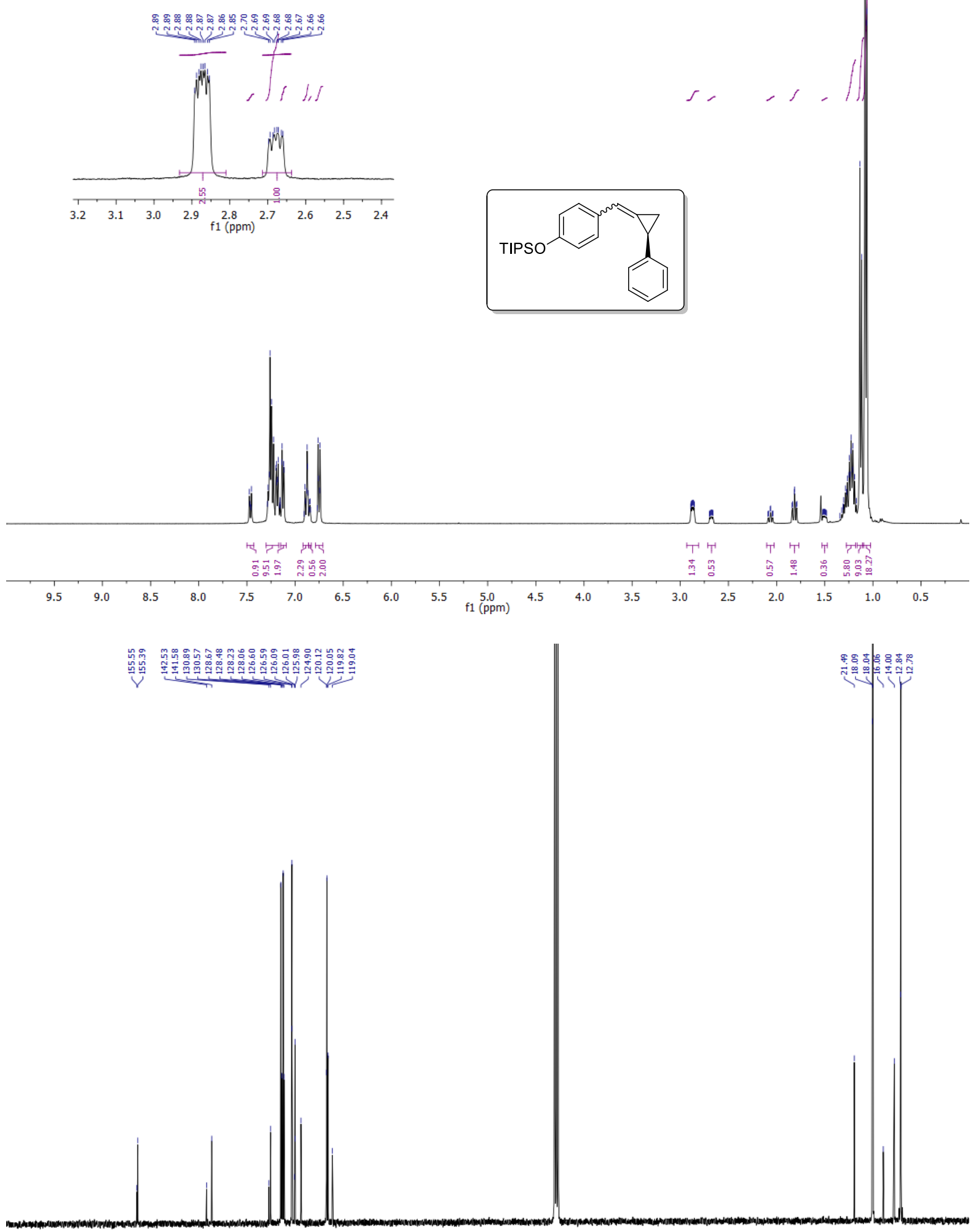


\section{$\underline{(R) \text {-(2-benzylidenecyclopropyl)benzene 6da }}$}

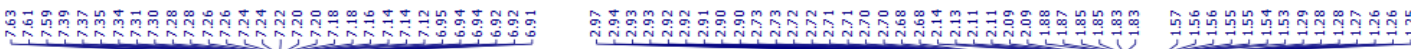
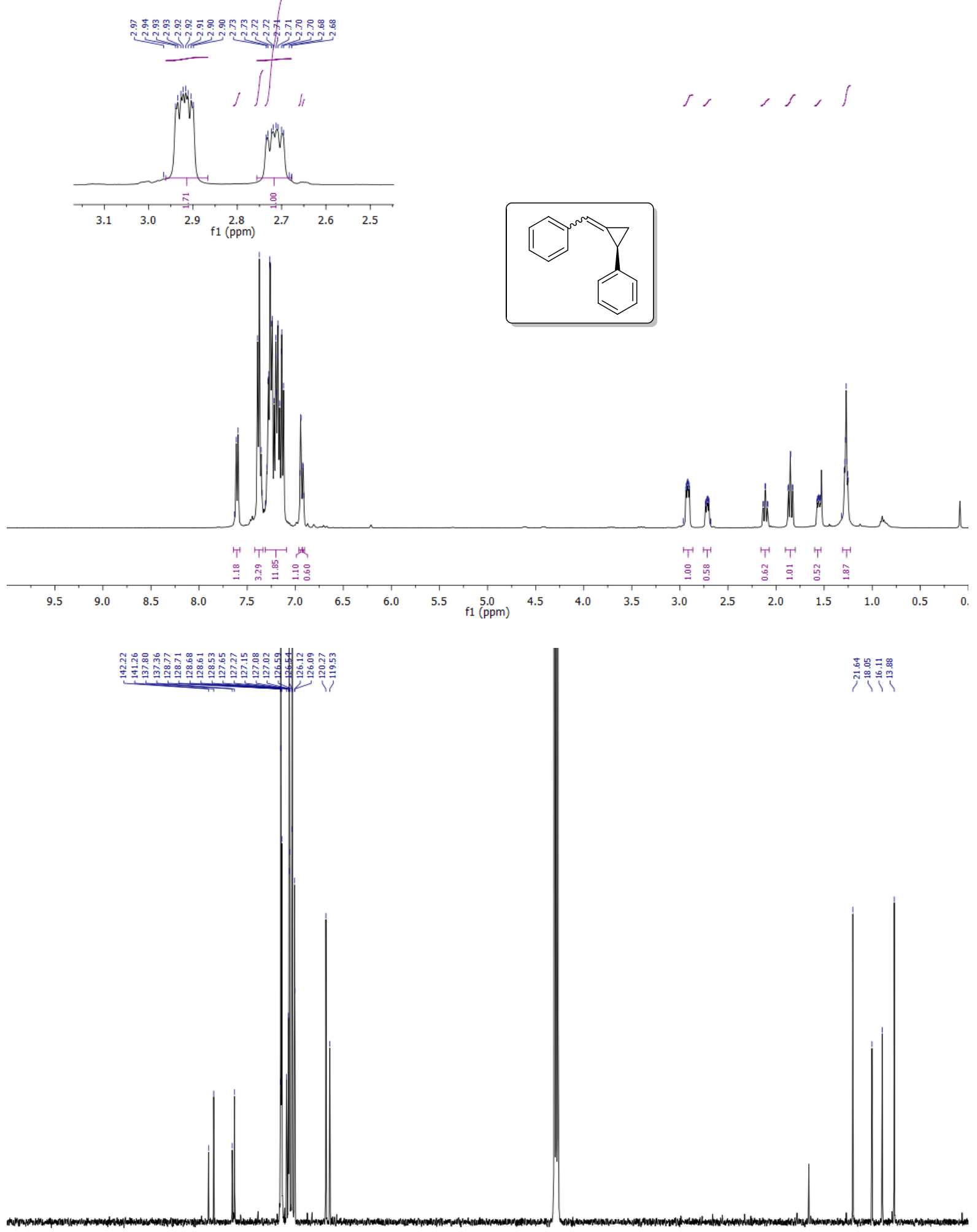

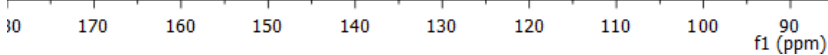

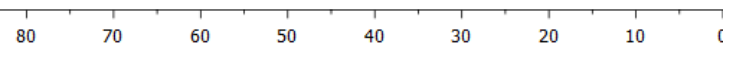



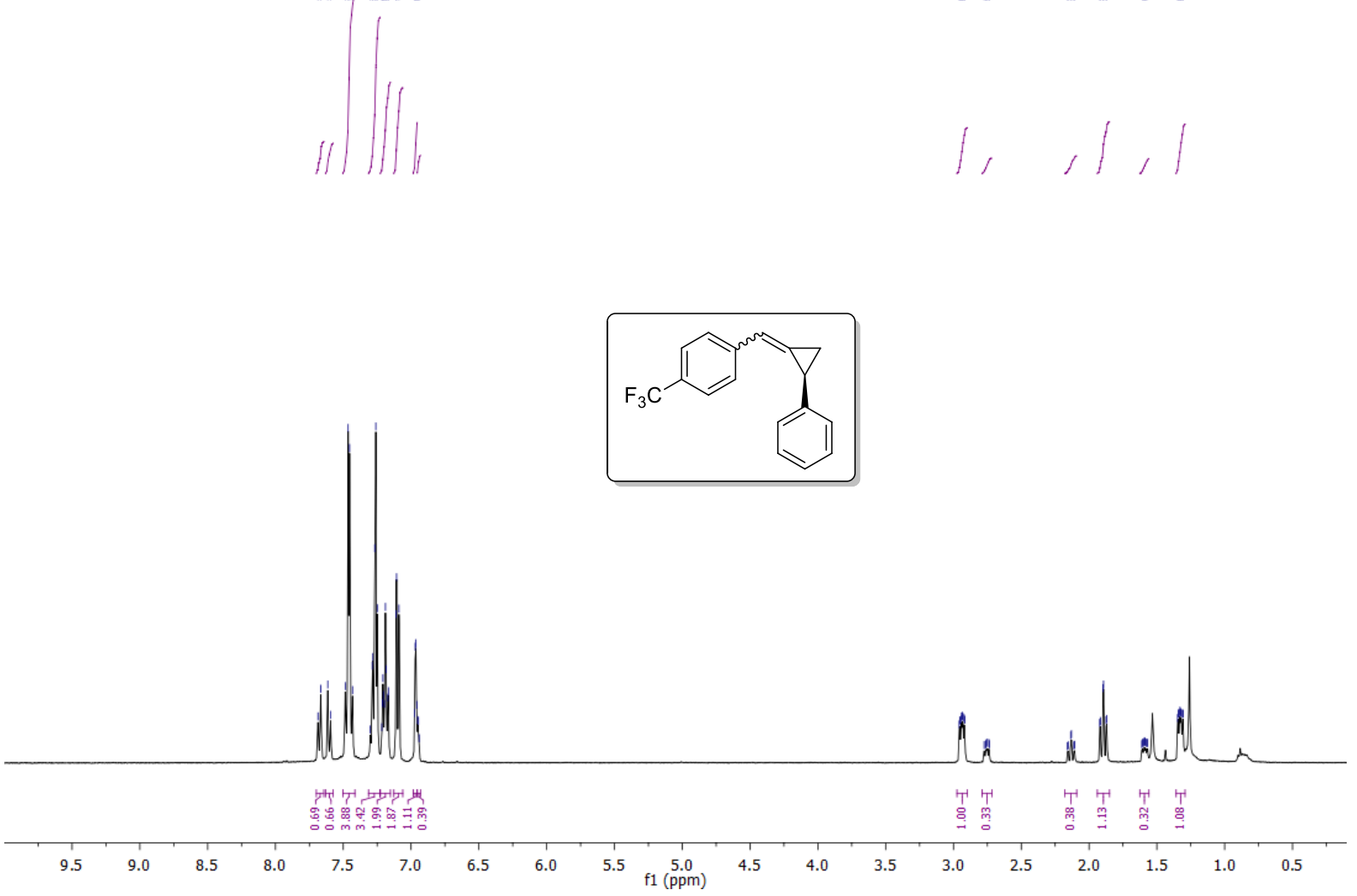

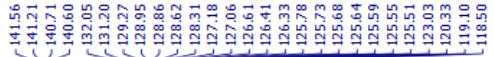
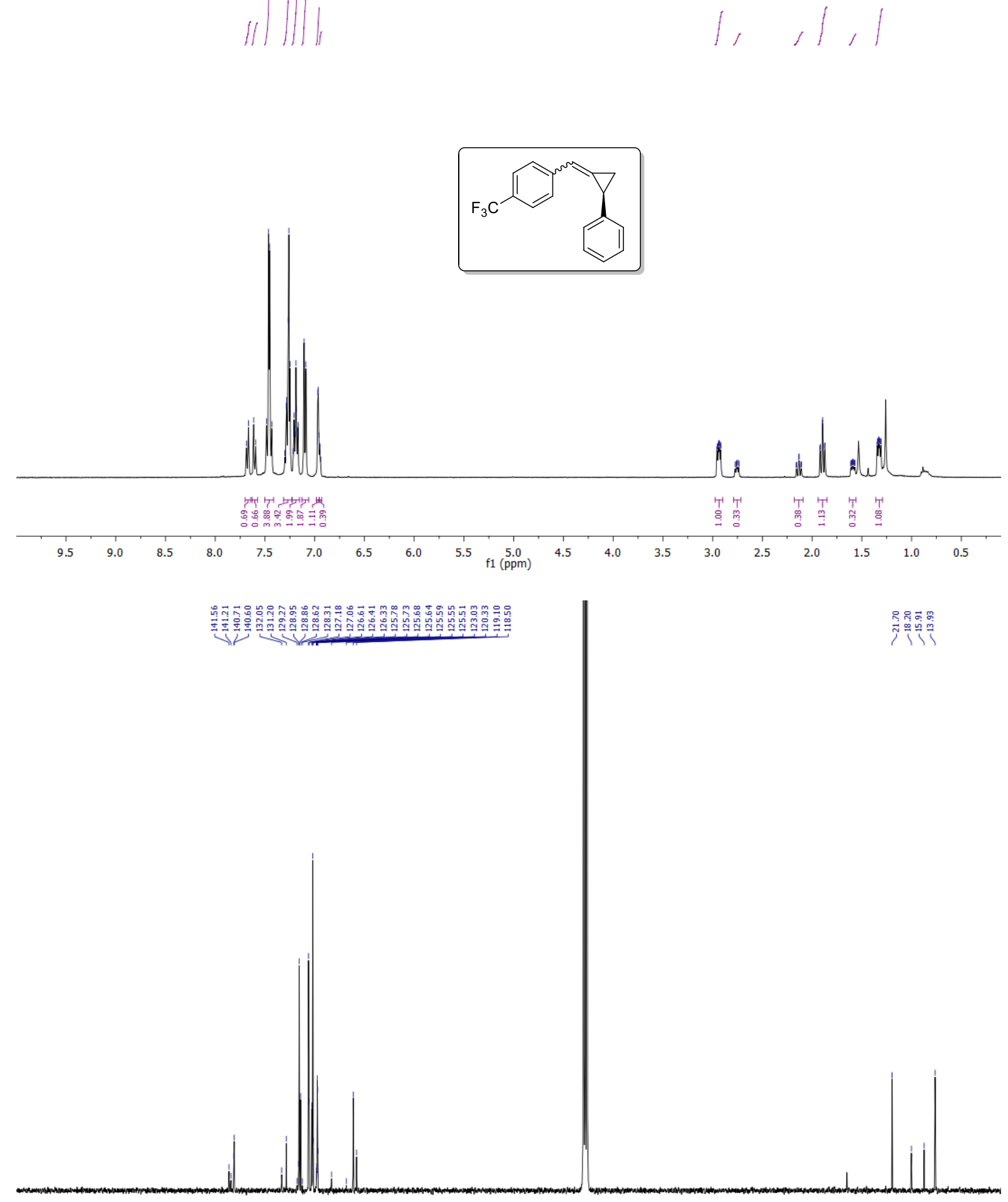$$
\text { . }
$$

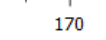

160

150

140

120

$100 \quad \begin{gathered}90 \\ \mathrm{f} 1(\mathrm{ppm})\end{gathered}$

80

70

10 
บั

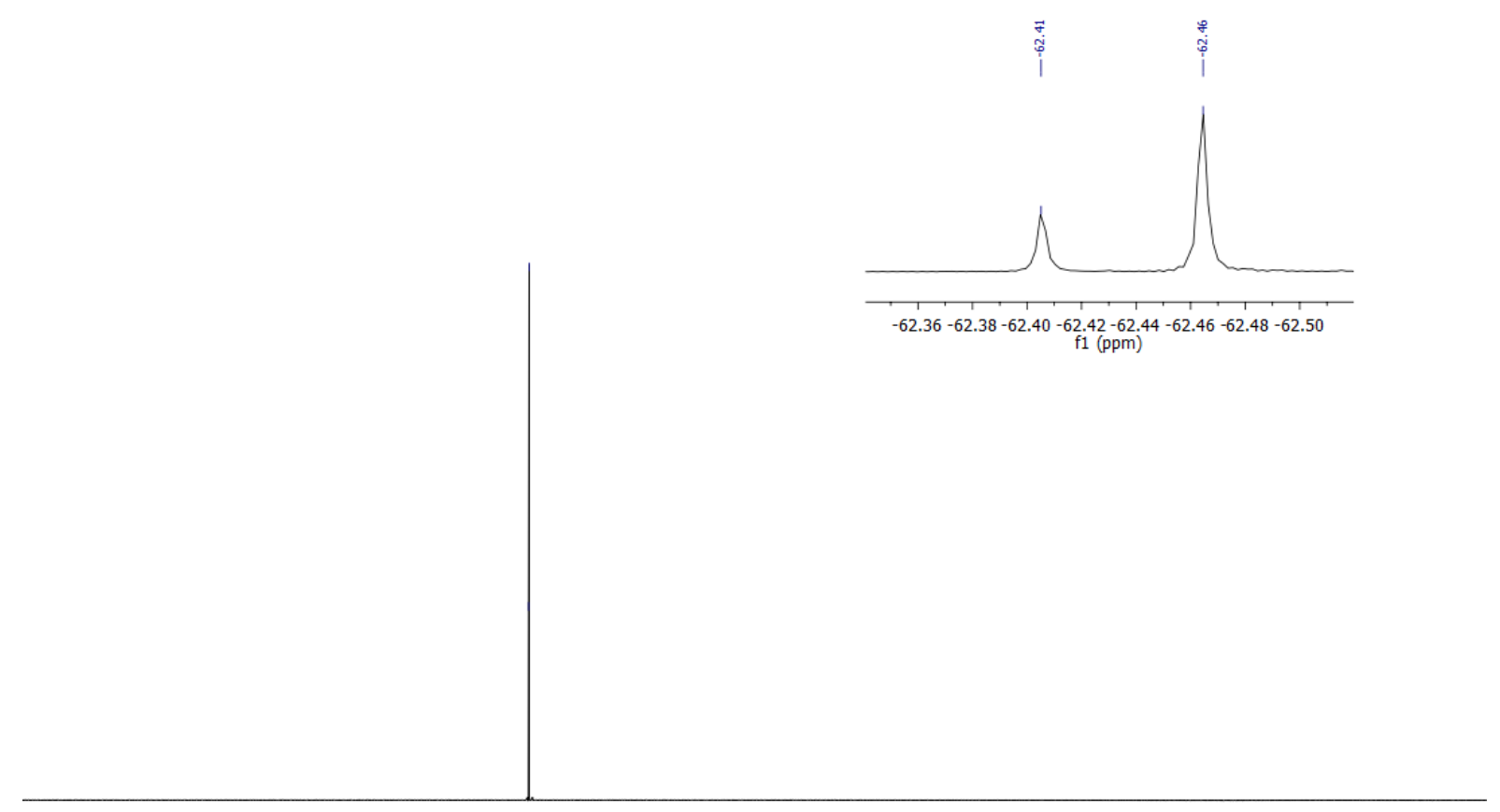$$
0
$$

0
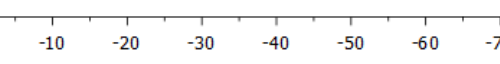

$\stackrel{-90}{\mathrm{f} 1}(\mathrm{ppm})$

$-110$

$\begin{array}{lll}130 & -14\end{array}$ 


\section{(R)-methyl 4-((2-phenylcyclopropylidene)methyl)benzoate 6fa}
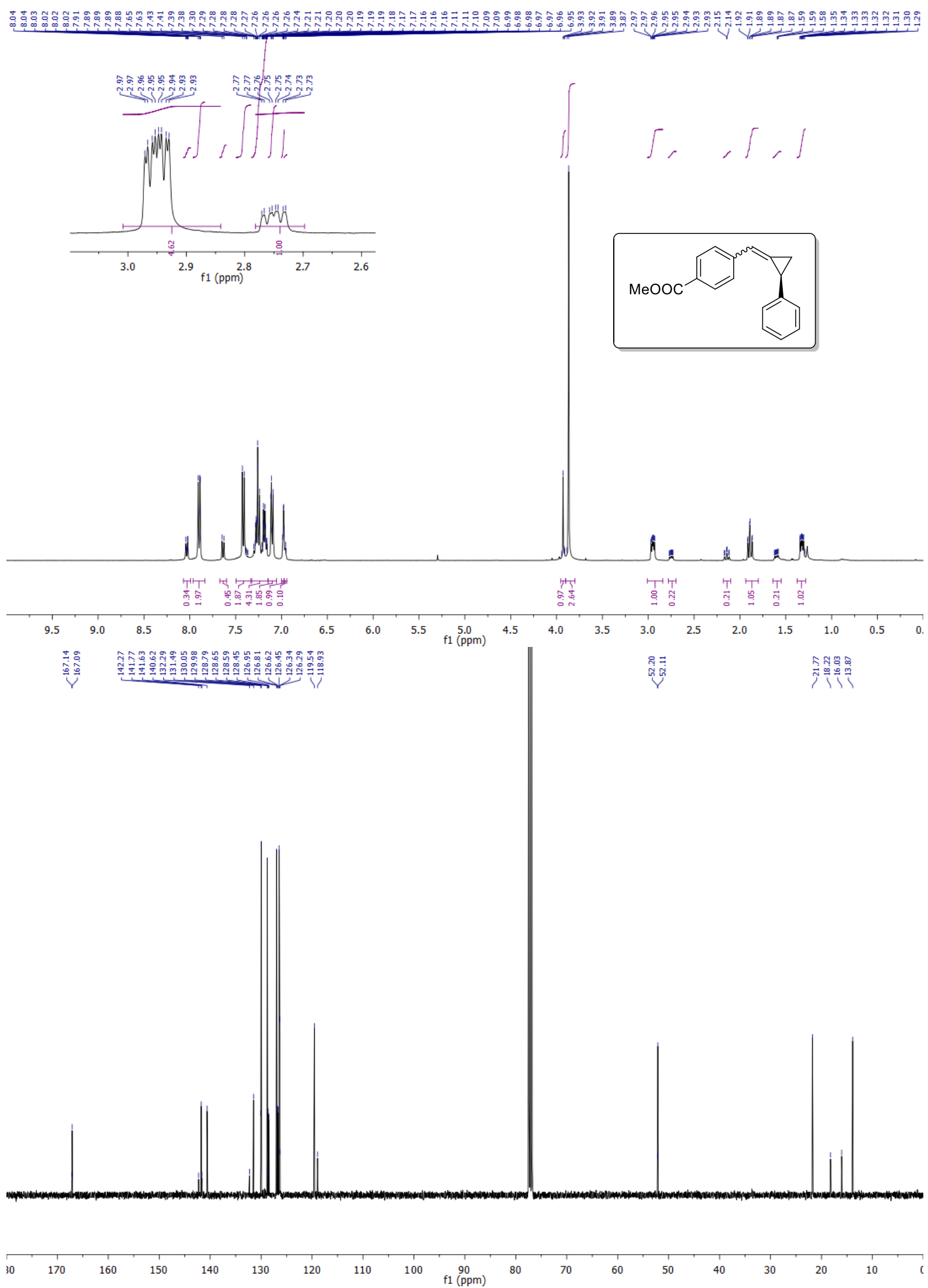
(R,Z)-methyl 4-((2-phenylcyclopropylidene)methyl)benzoate $(R, Z)-6 f a$

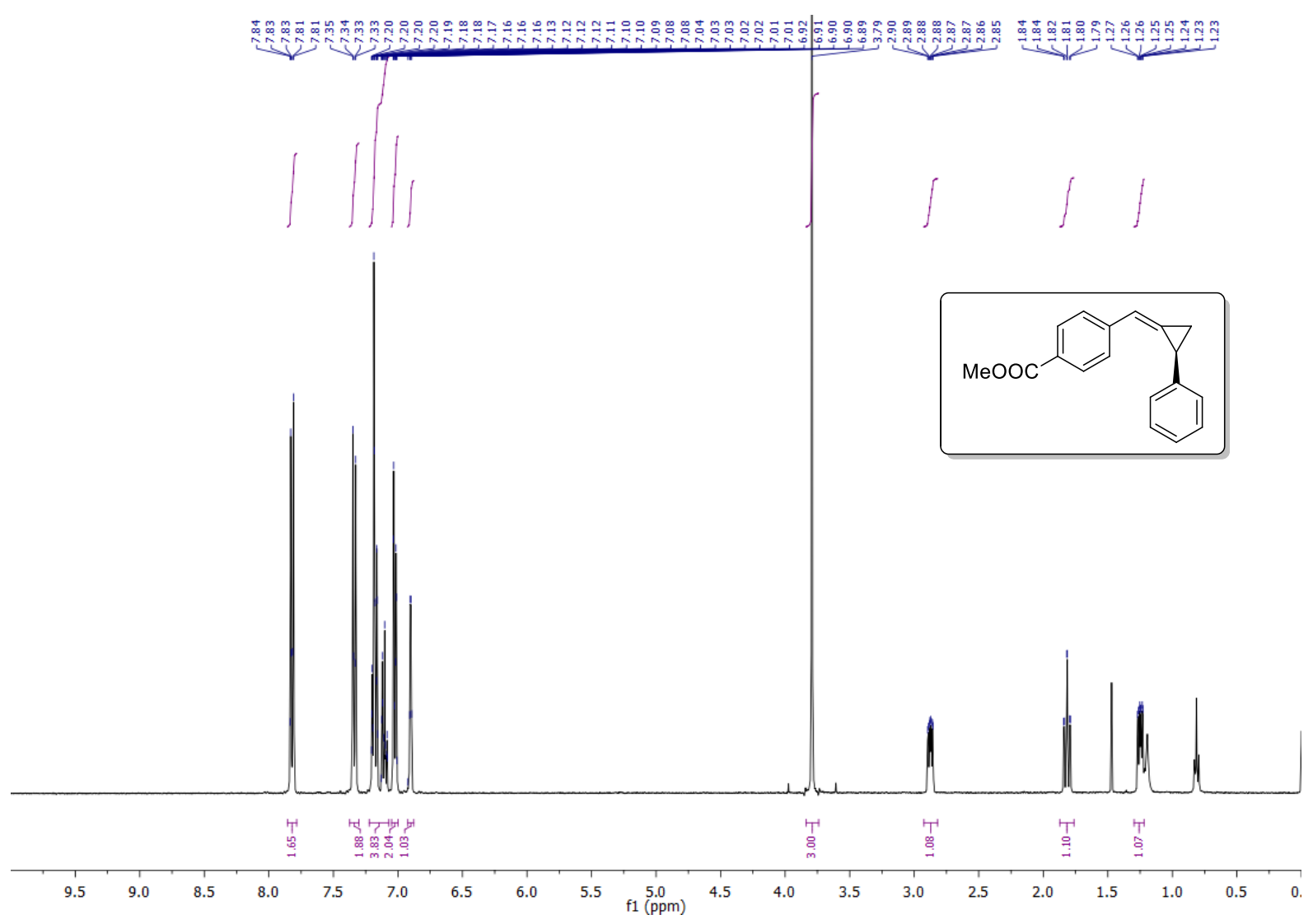

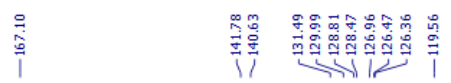

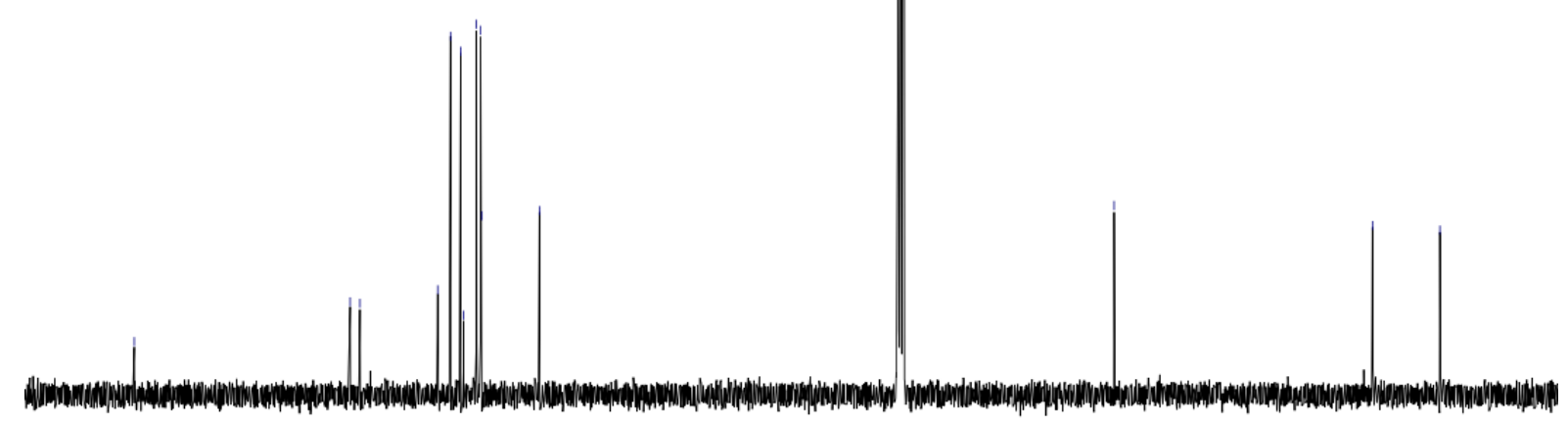

$\begin{array}{lllllllll}170 & 160 & 150 & 140 & 130 & 120 & 110 & 100 & \begin{array}{c}90 \\ \text { f1 }(\mathrm{ppm})\end{array}\end{array}$

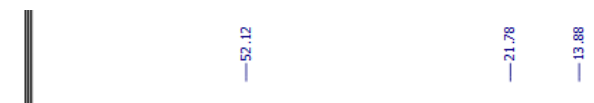




\section{$\underline{(E) \text {-methyl 4-((2-phenylcyclopropylidene)methyl)benzoate }(E) \text {-6fa }}$}
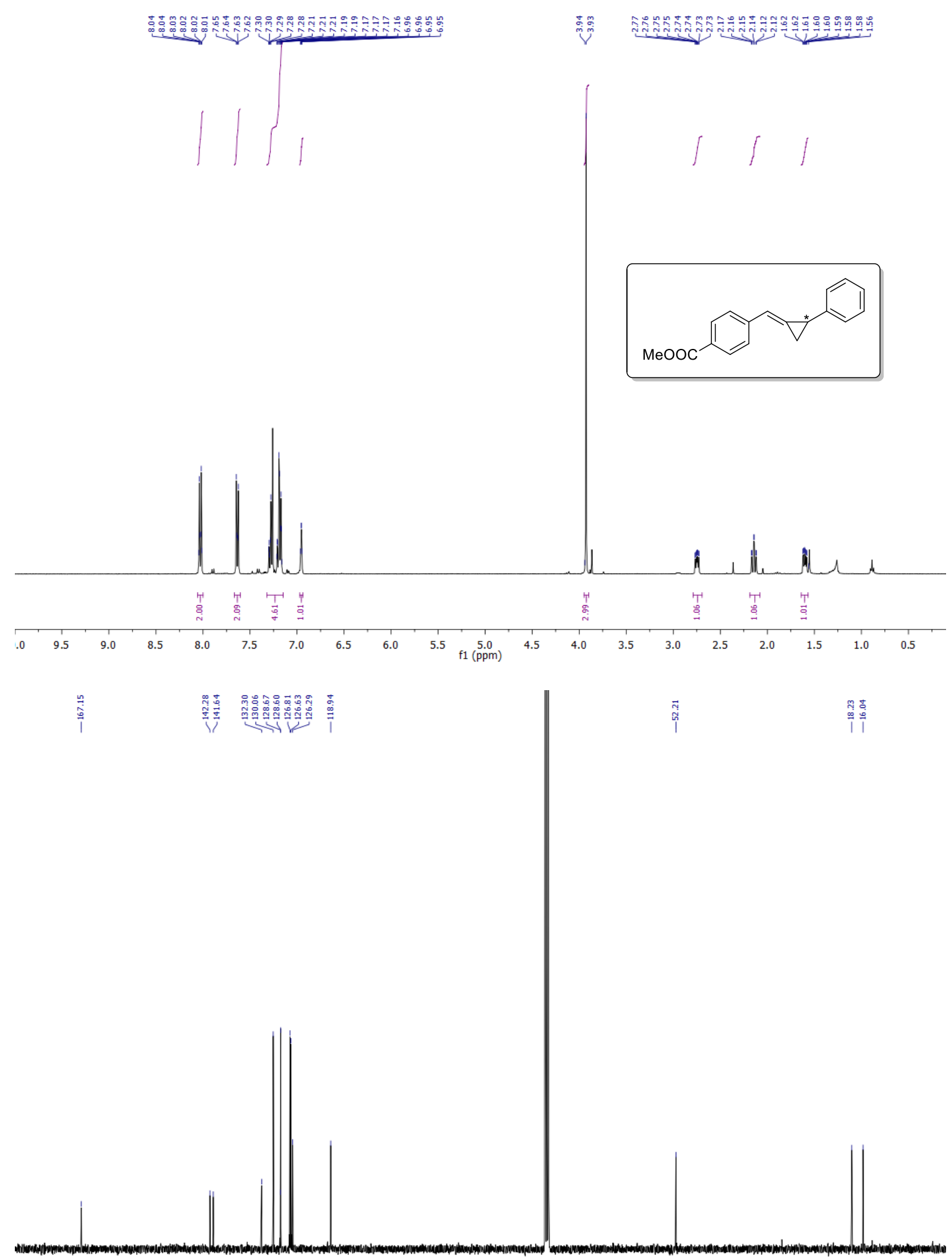

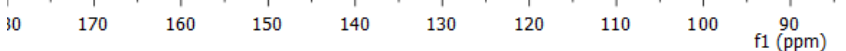

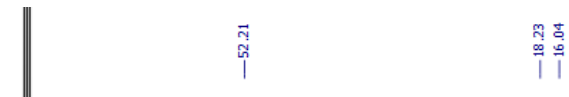




\section{(R)-1-fluoro-4-((2-phenylcyclopropylidene)methyl)benzene 6ga}

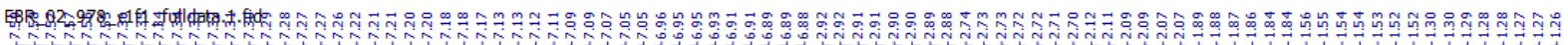
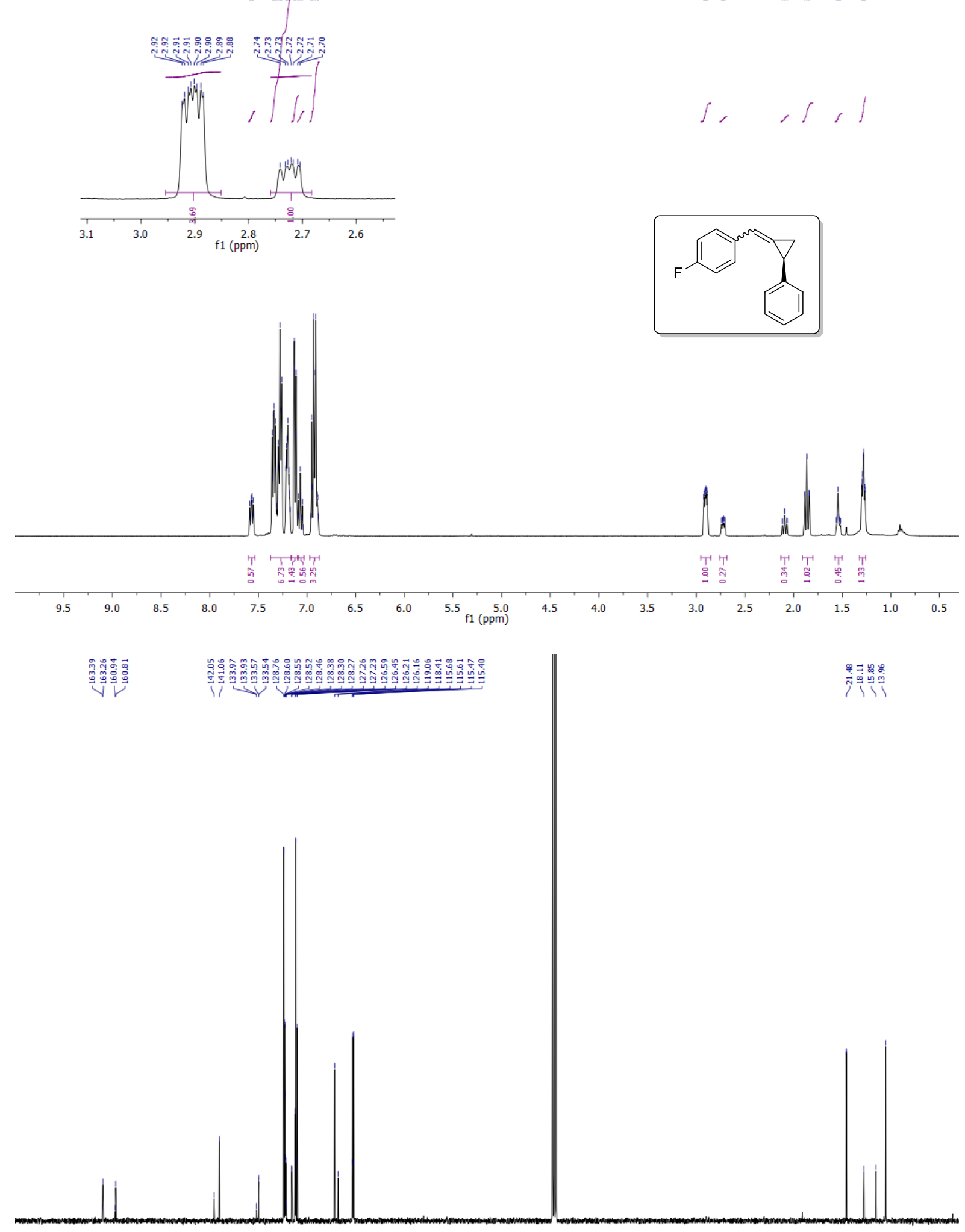

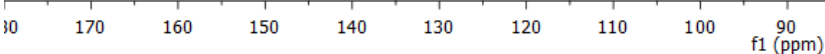

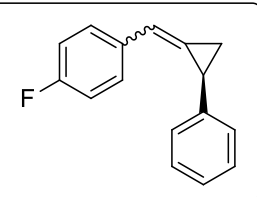




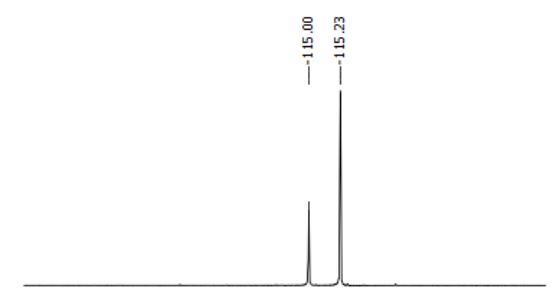

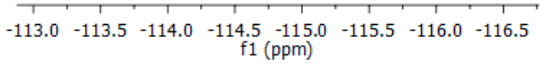

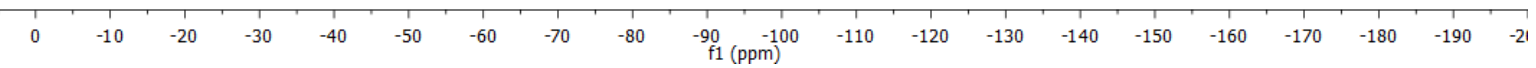




\section{(R)-2-((2-phenylcyclopropylidene)methyl)naphthalene 6ha}

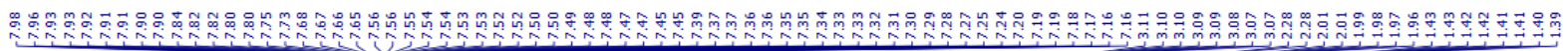
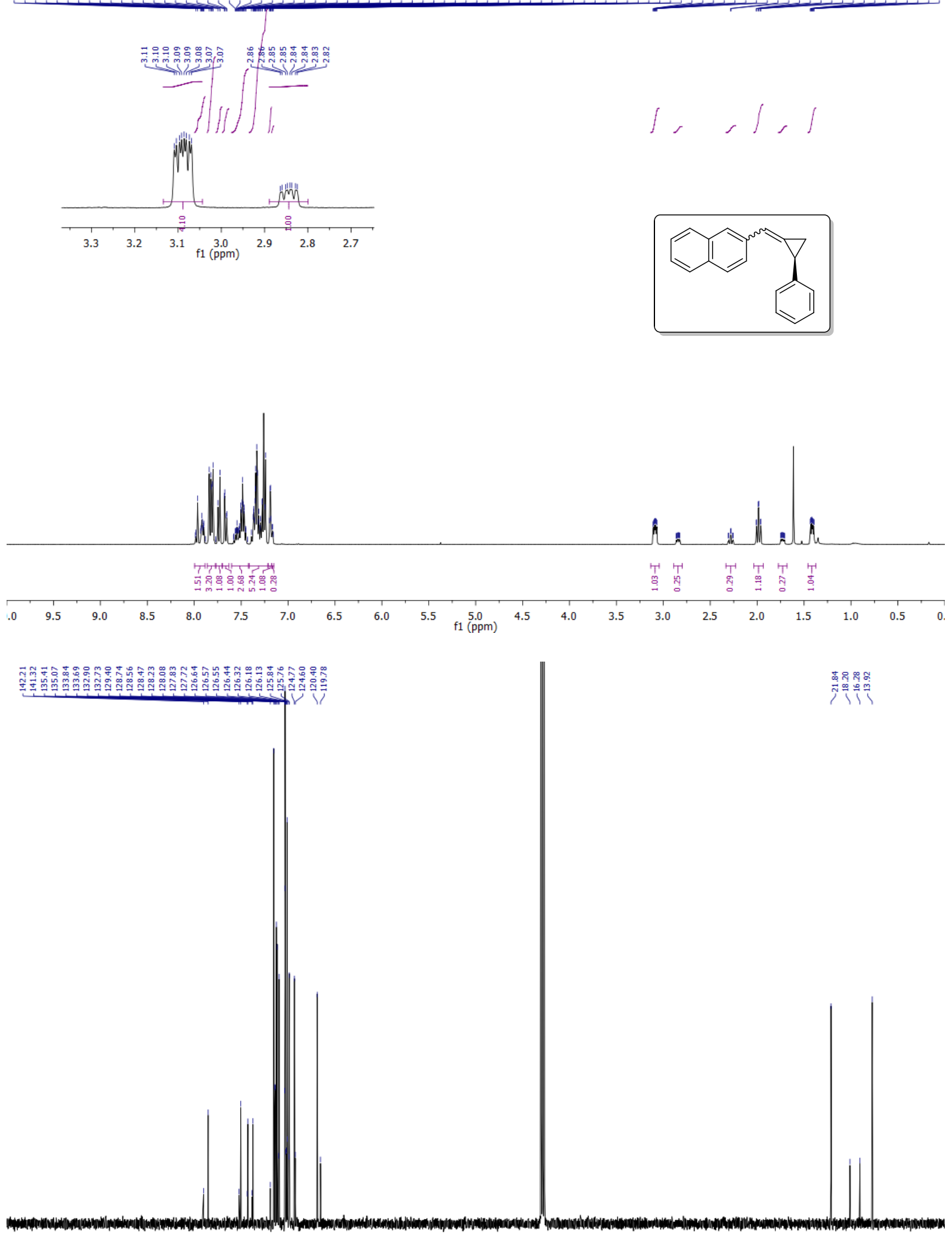

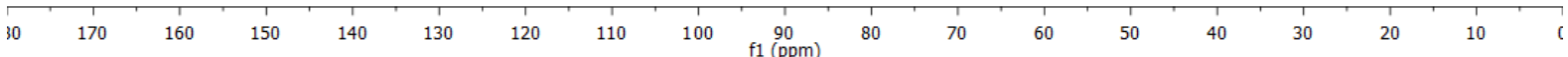




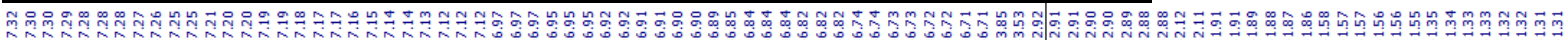

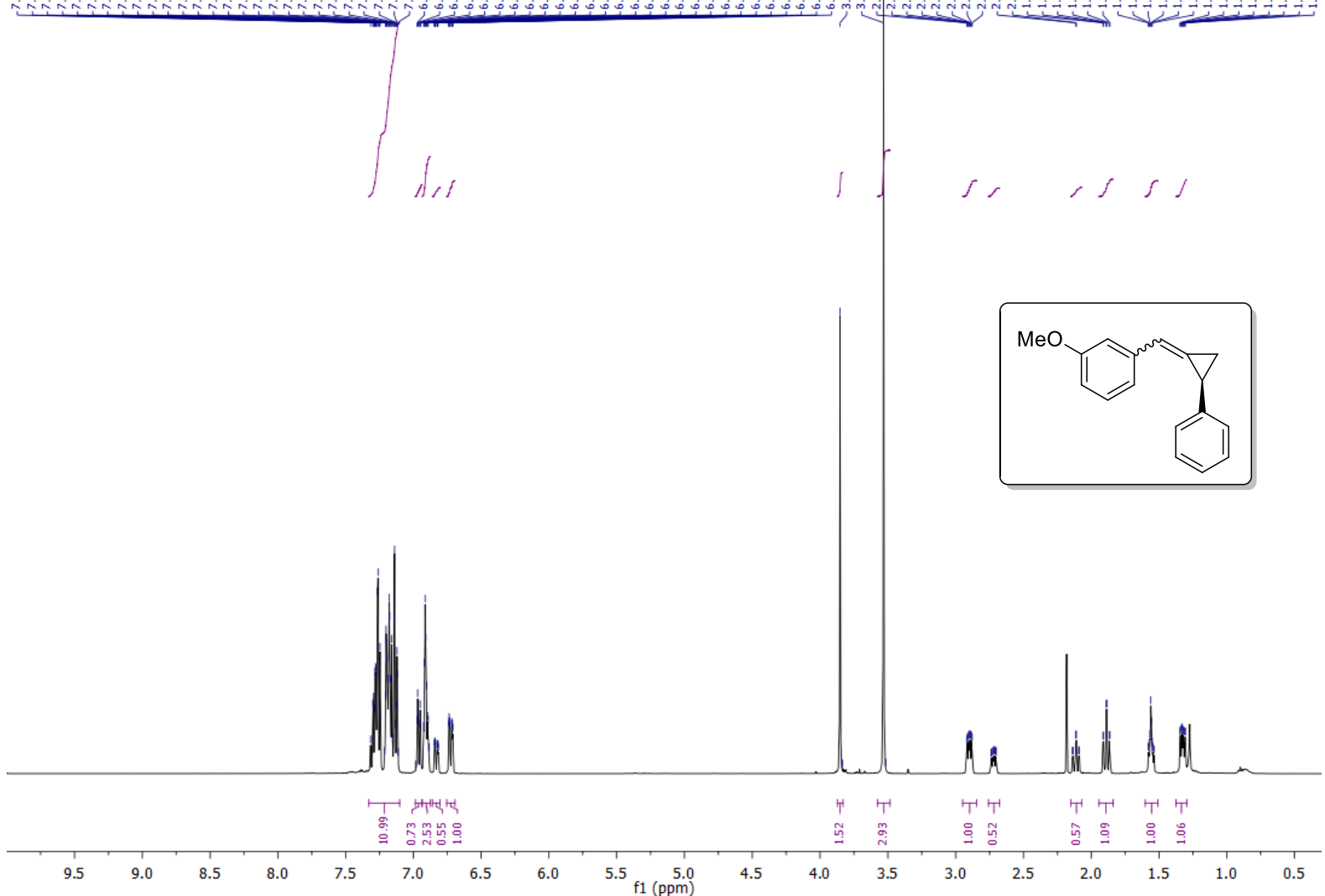

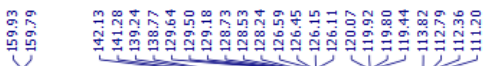
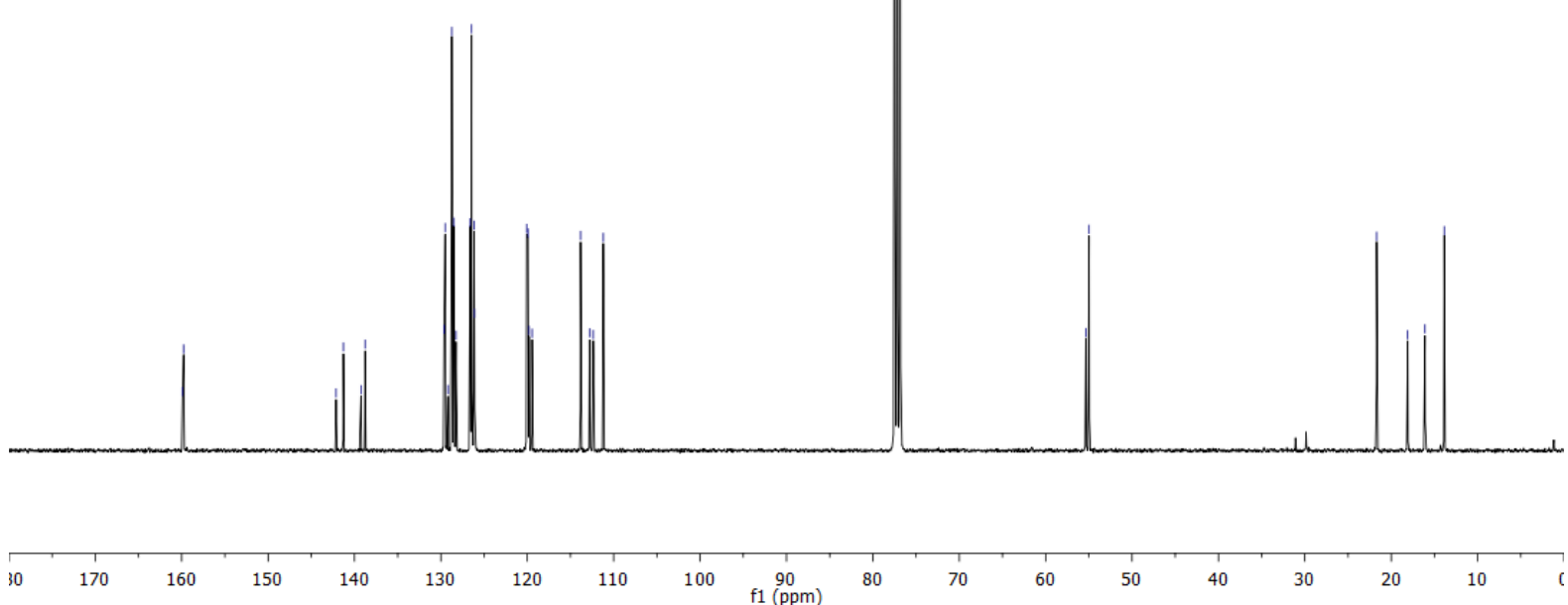


\section{(R)-1-methoxy-2-((2-phenylcyclopropylidene)methyl)benzene 6ja}

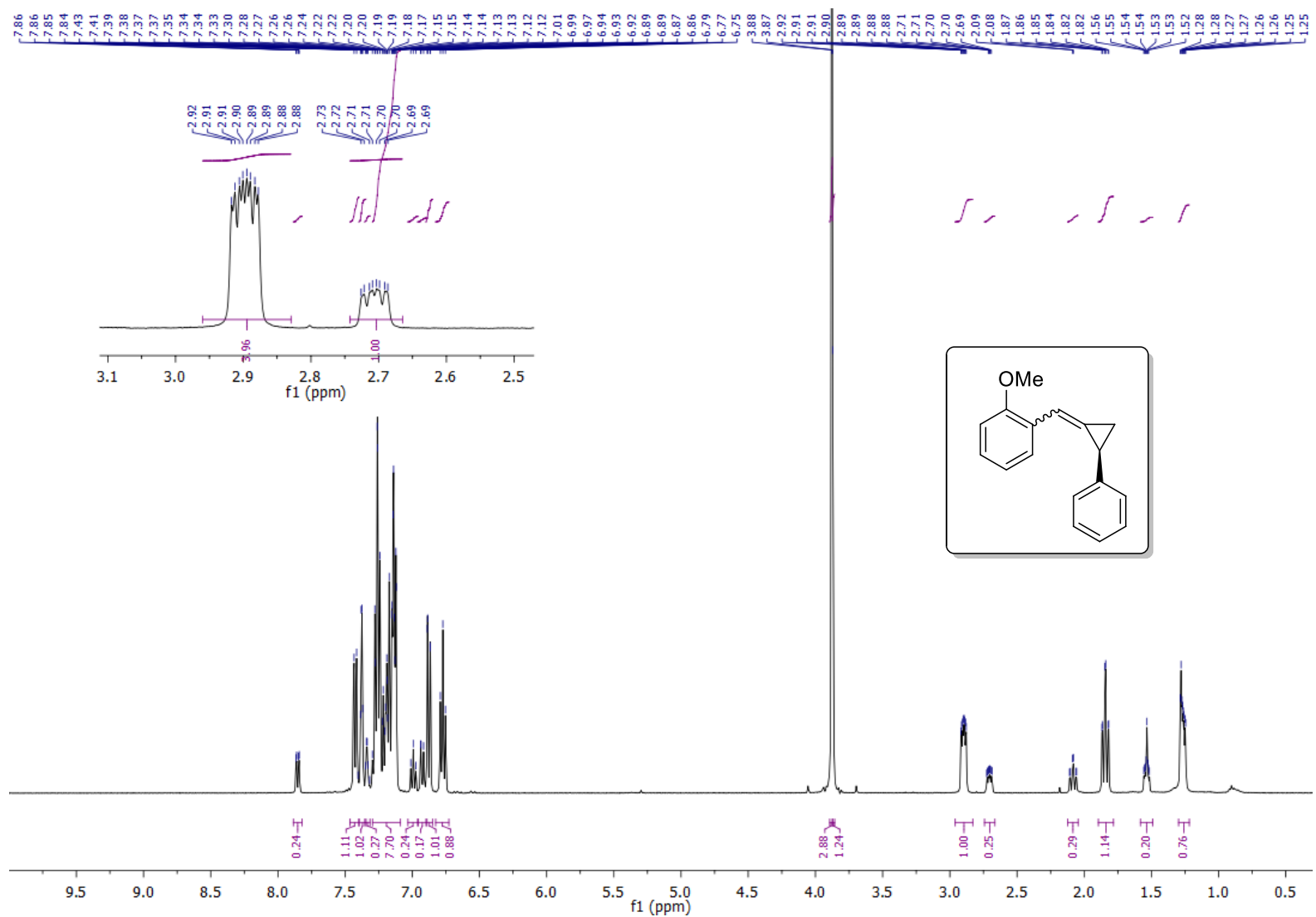

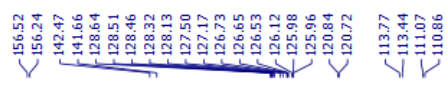

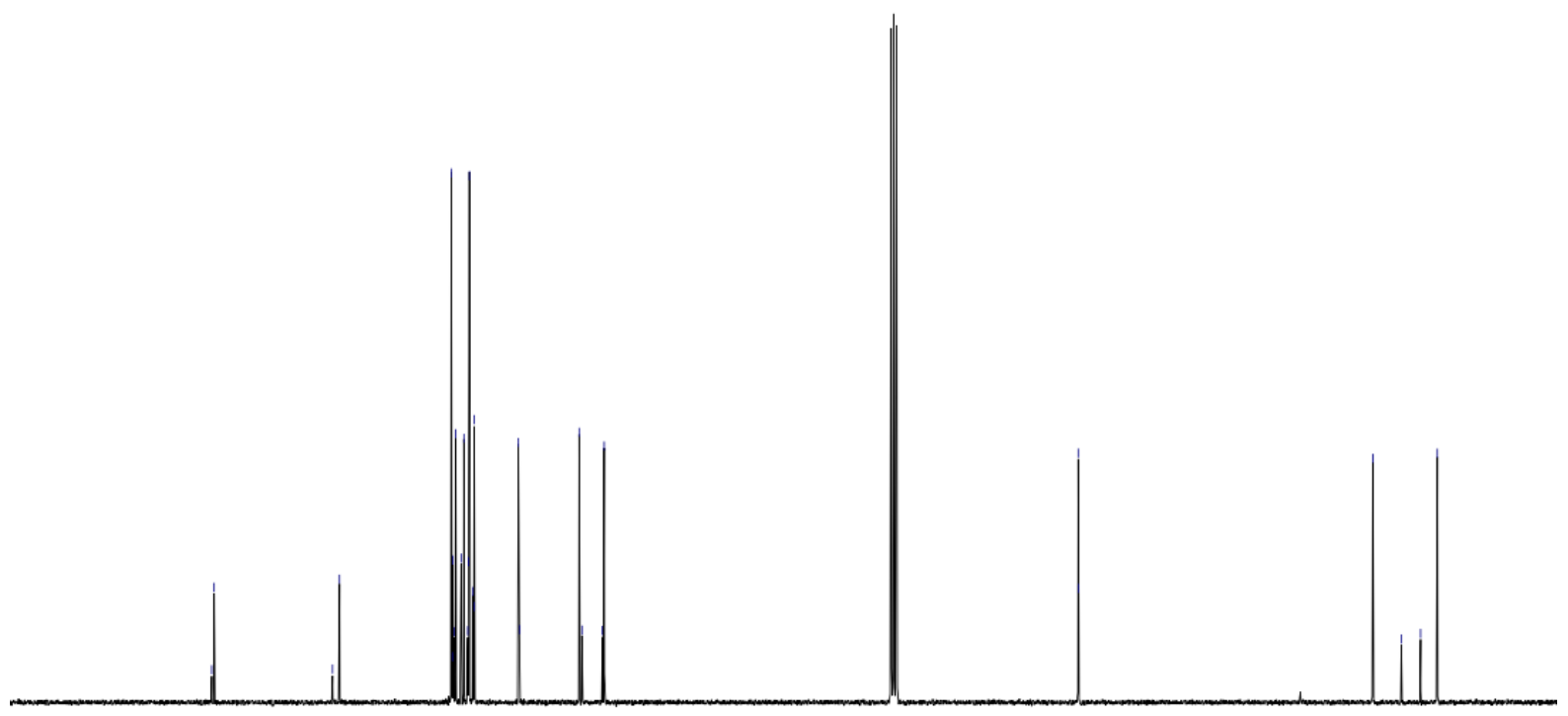

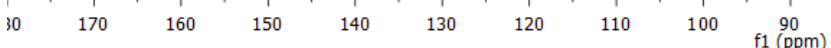
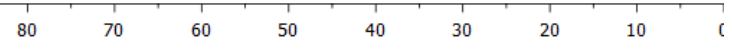


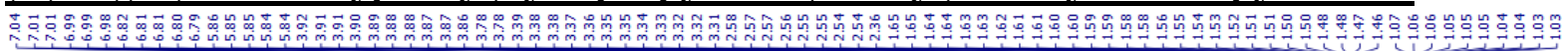
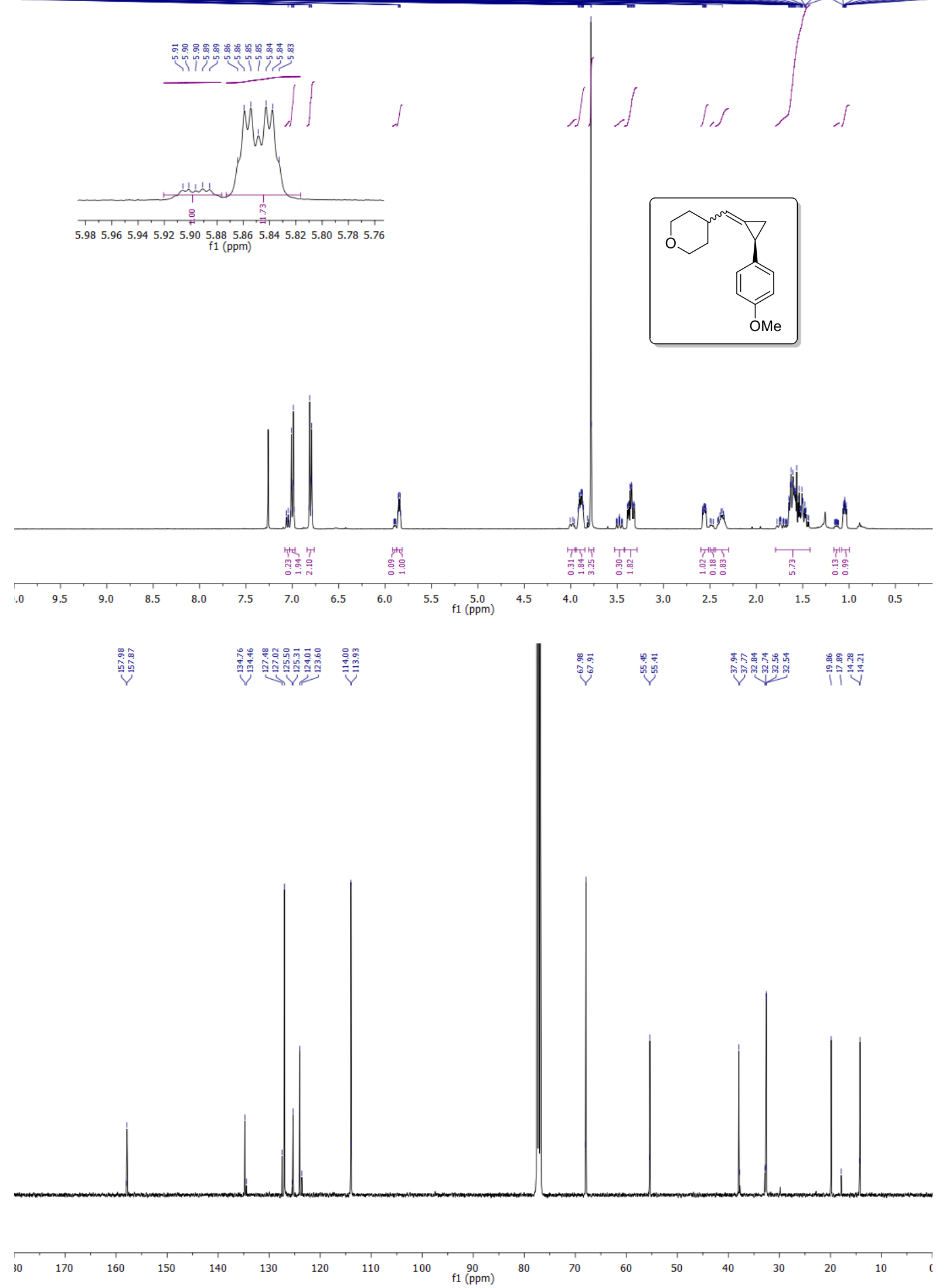

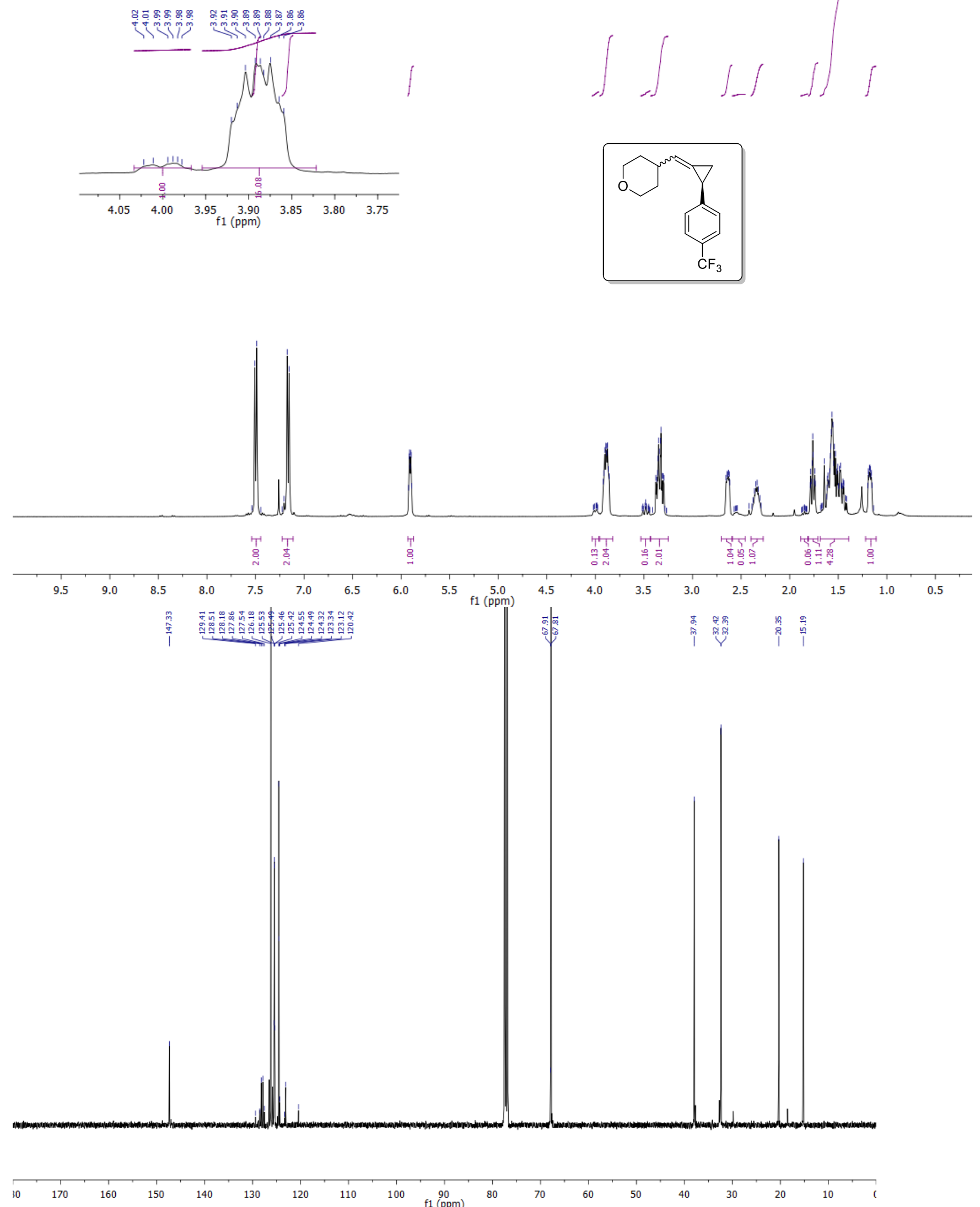

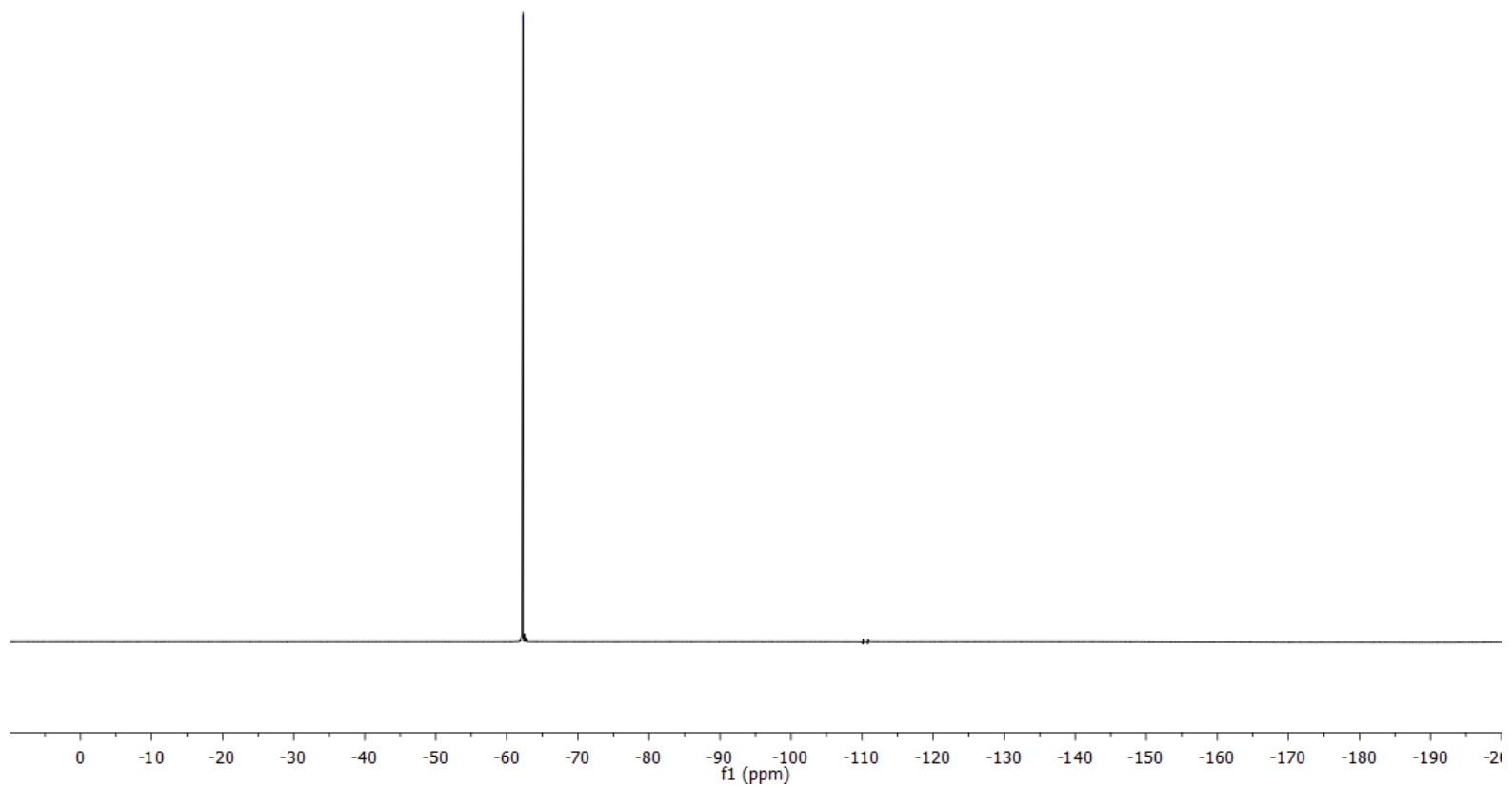


\section{$\underline{(R)-2-(2-(4-m e t h o x y b e n z y l i d e n e) c y c l o p r o p y l) n a p h t h a l e n e ~ 6 a d ~}$}

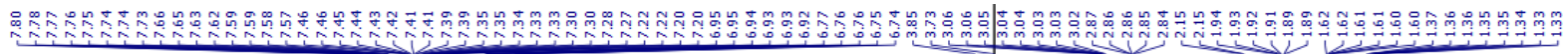
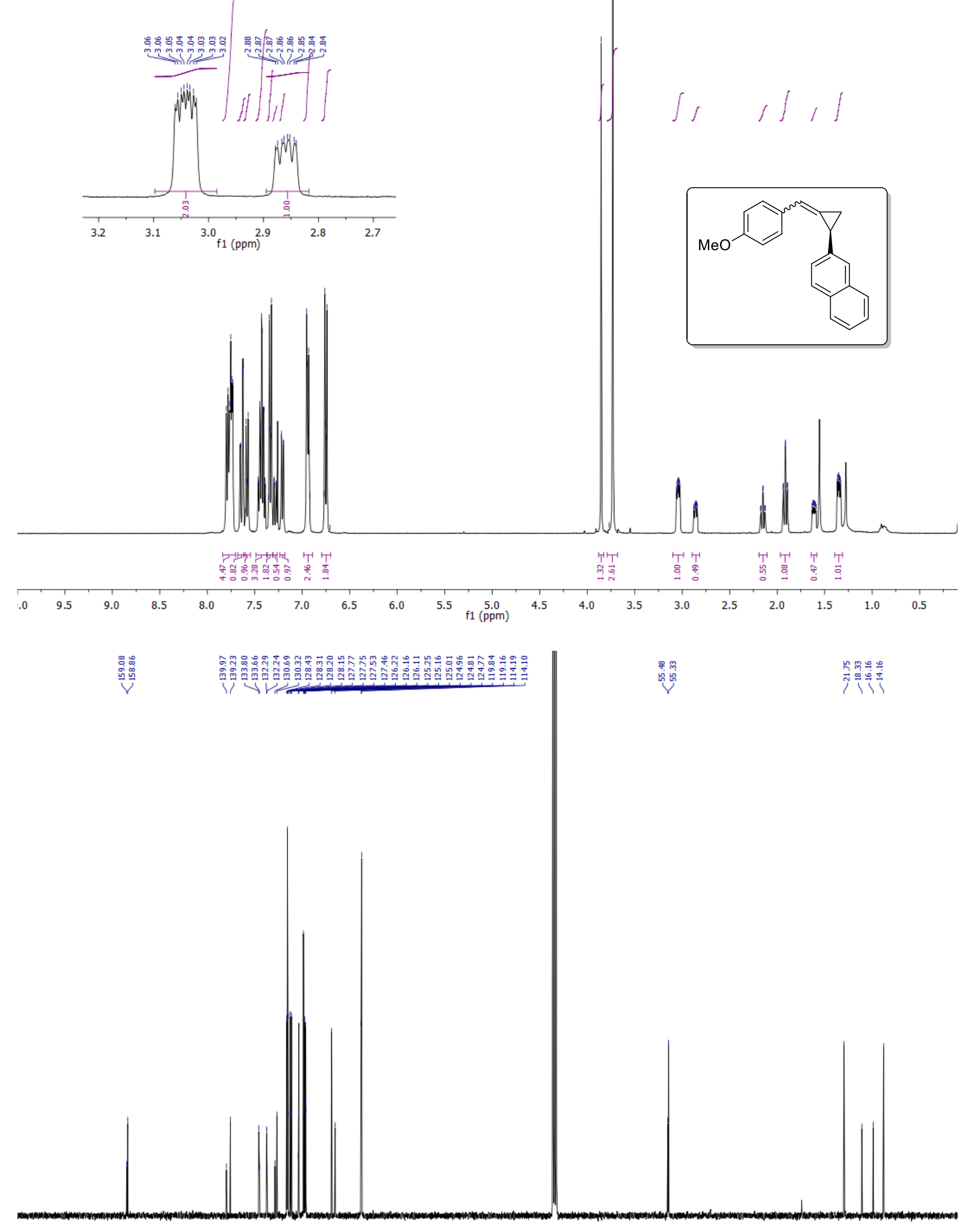


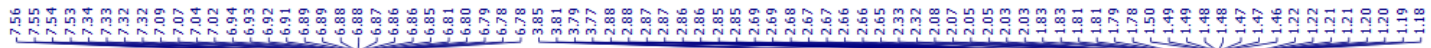
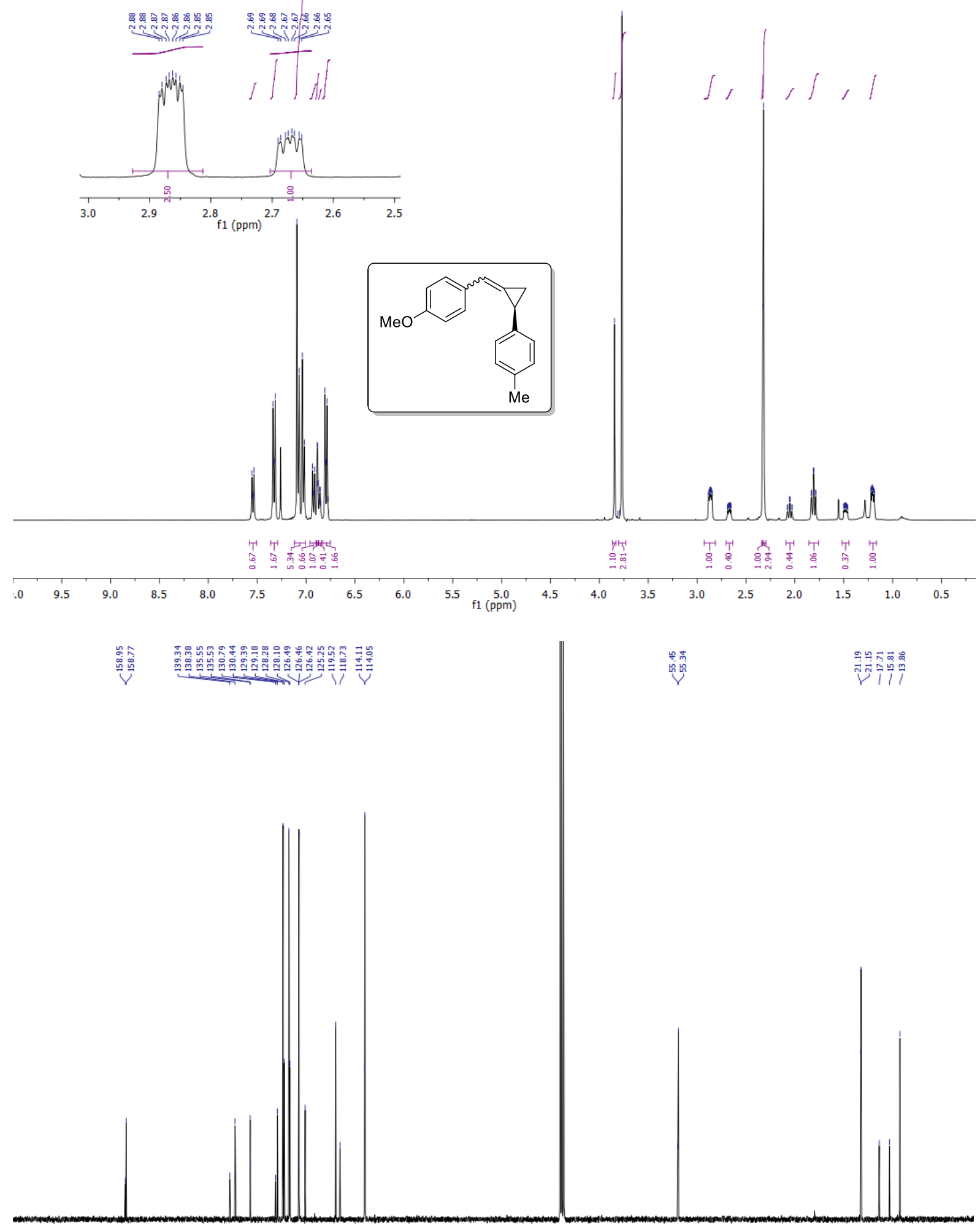

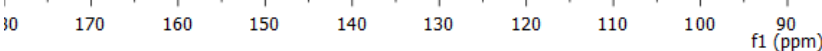




\section{$\underline{(R) \text {-methyl 4-(2-(4-methoxybenzylidene)cyclopropyl)benzoate 6af }}$}

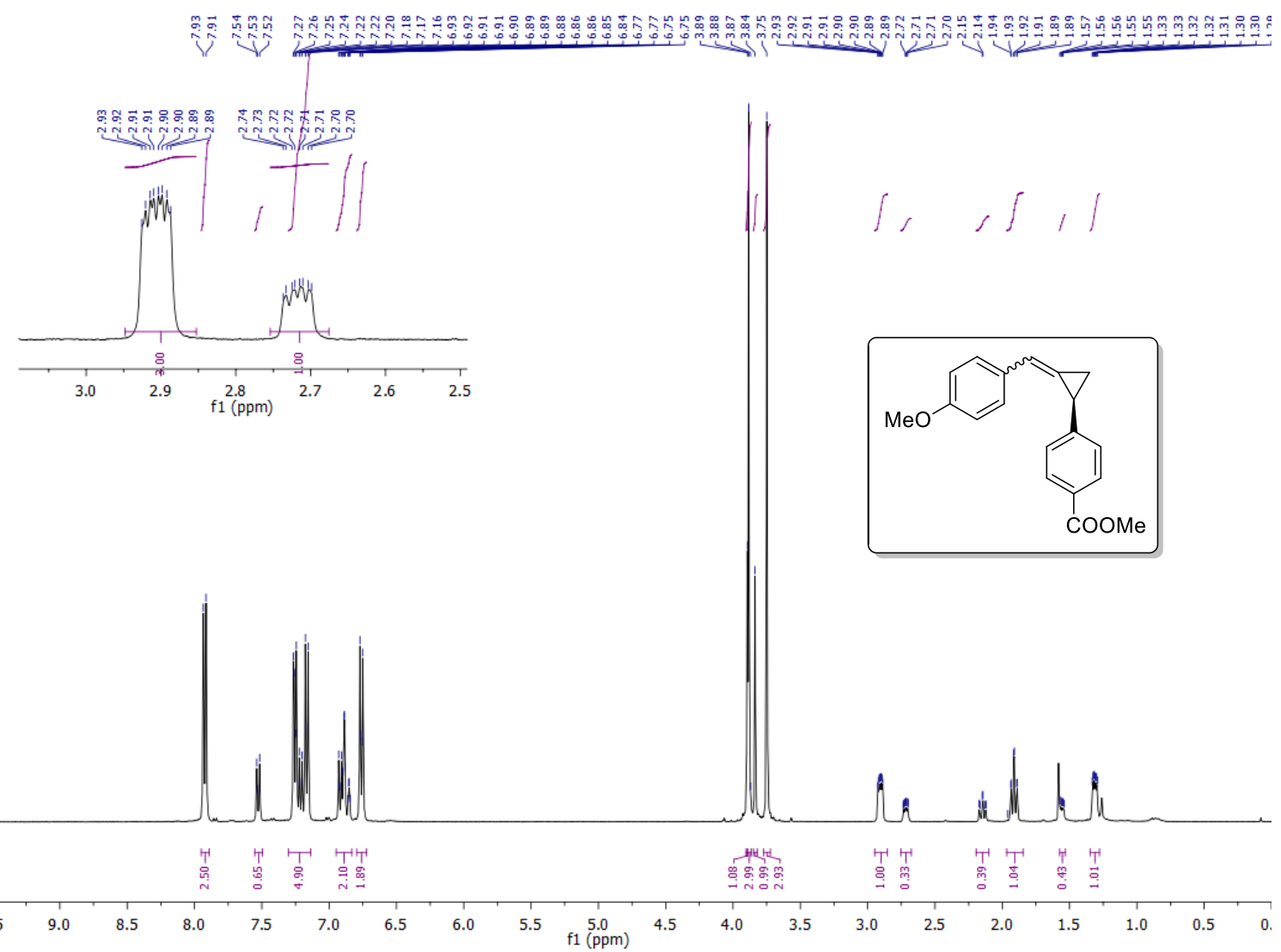

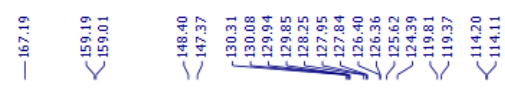

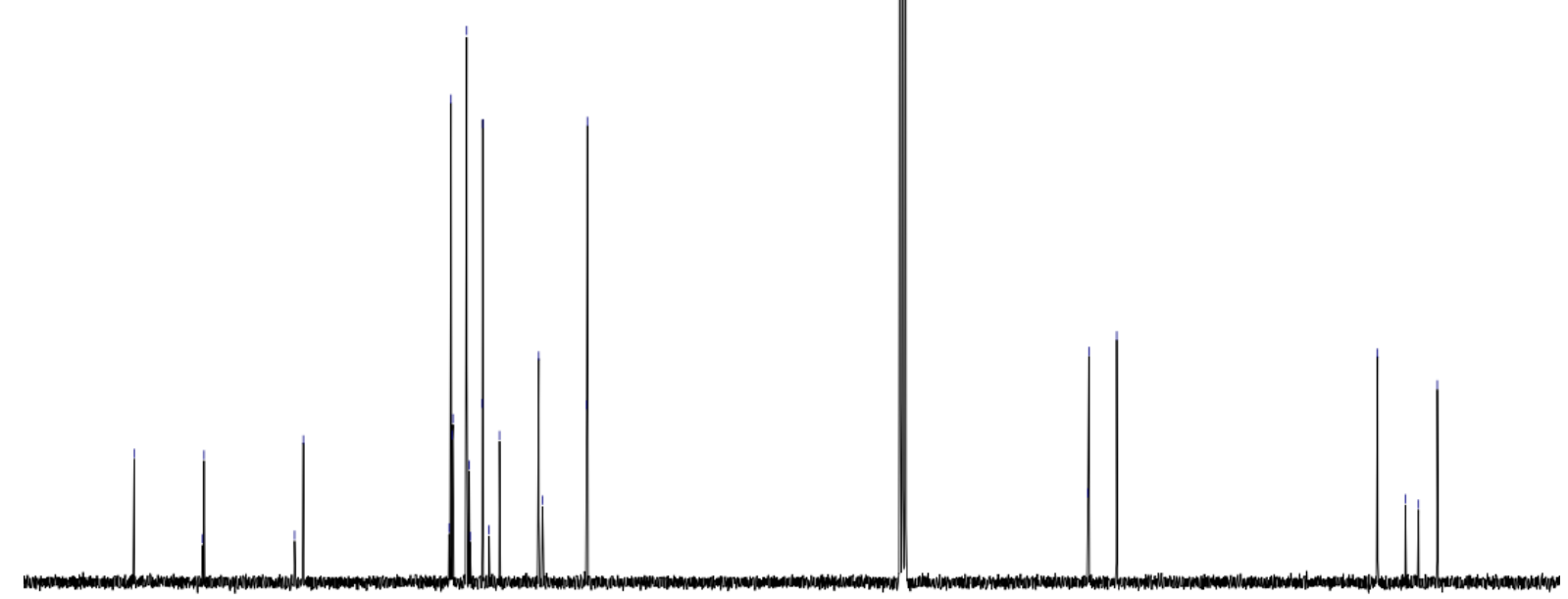




\section{(R)-1-chloro-4-(2-(4-methoxybenzylidene)cyclopropyl)benzene 6ag}

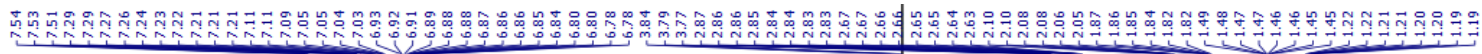
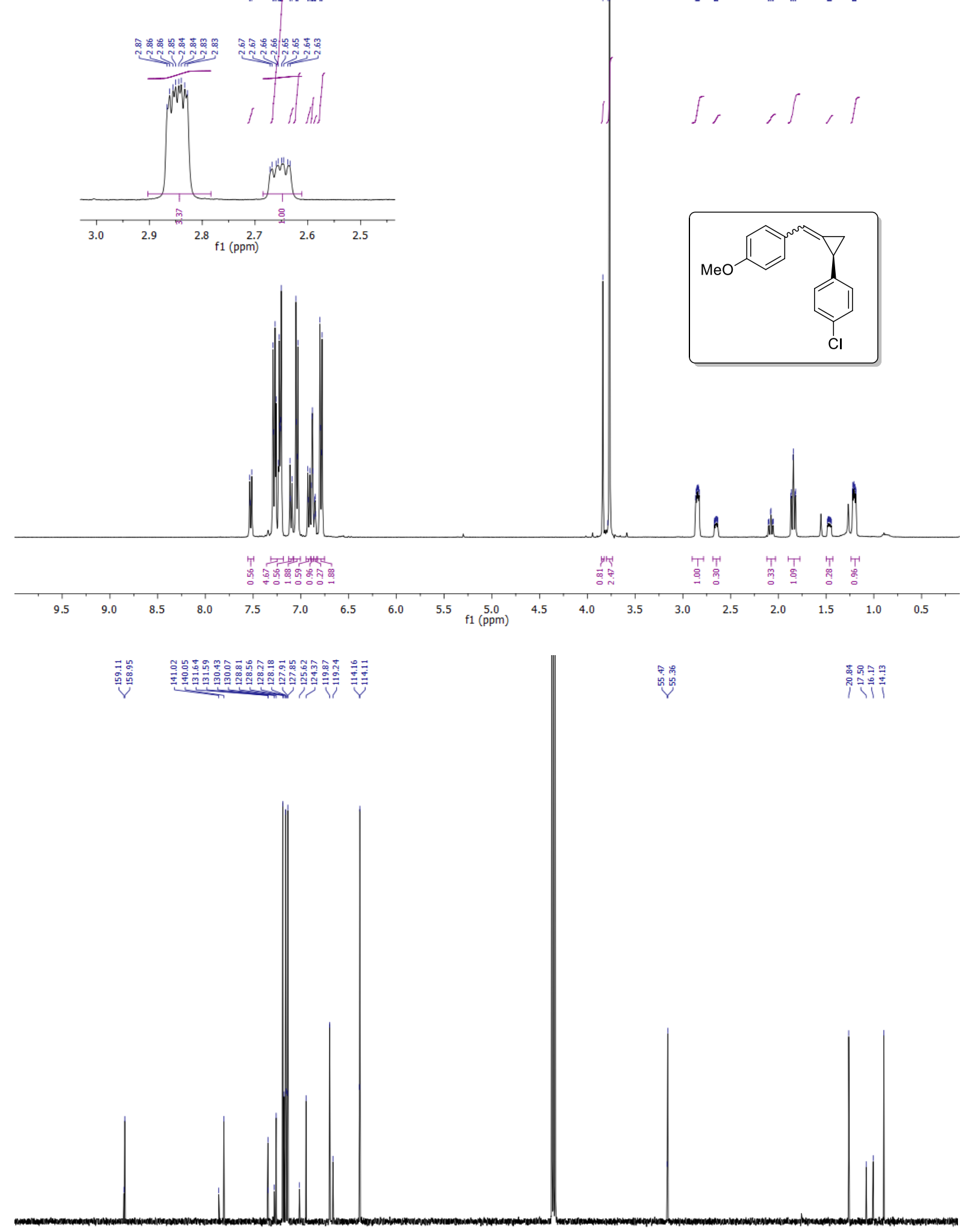

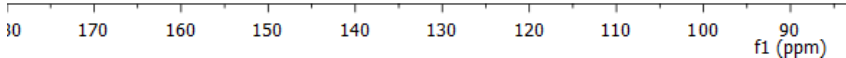

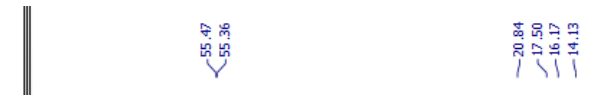




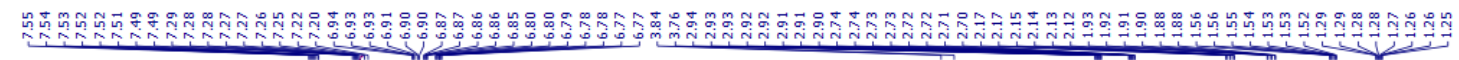
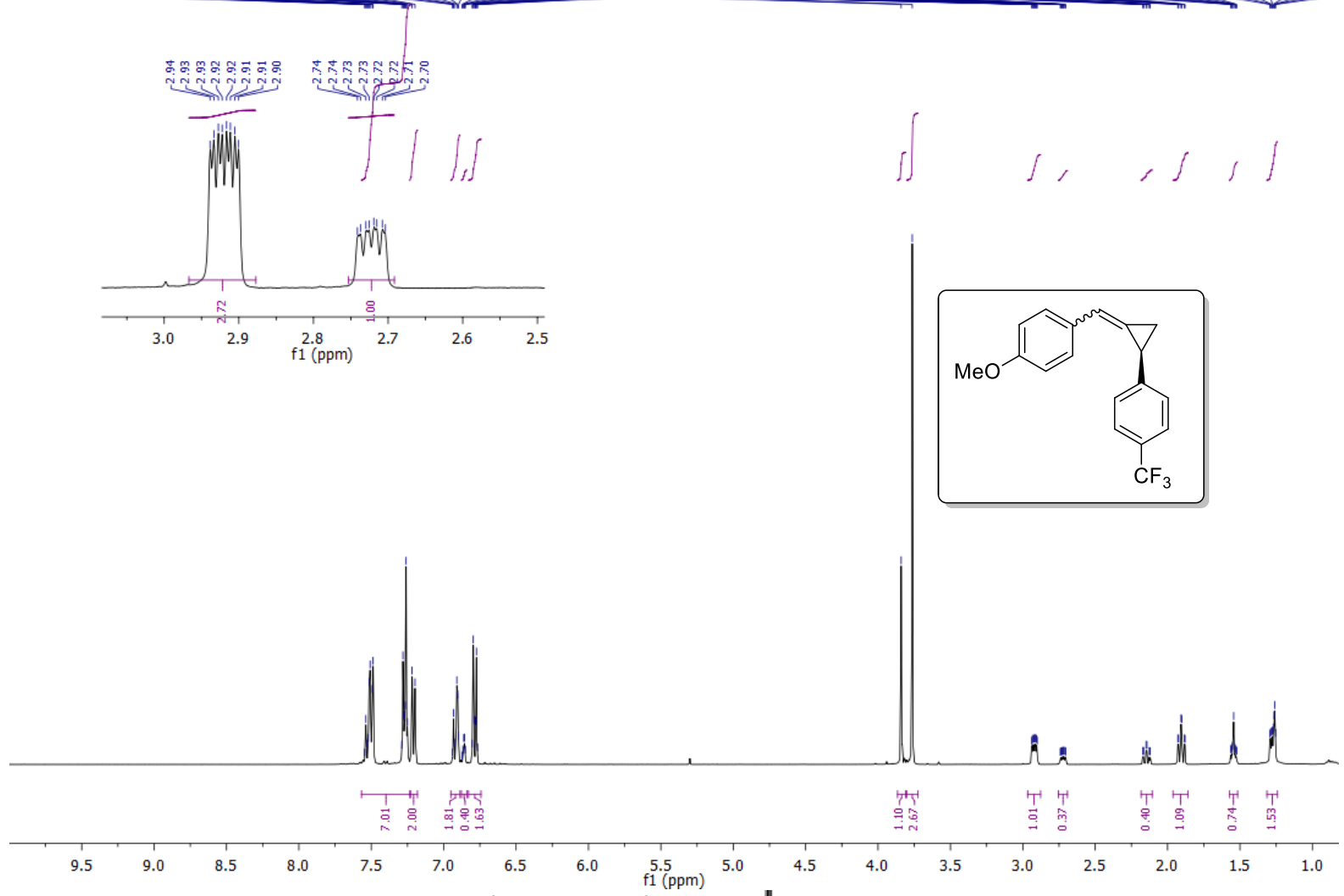

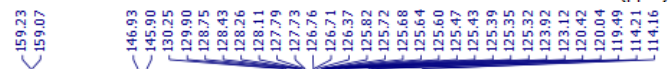

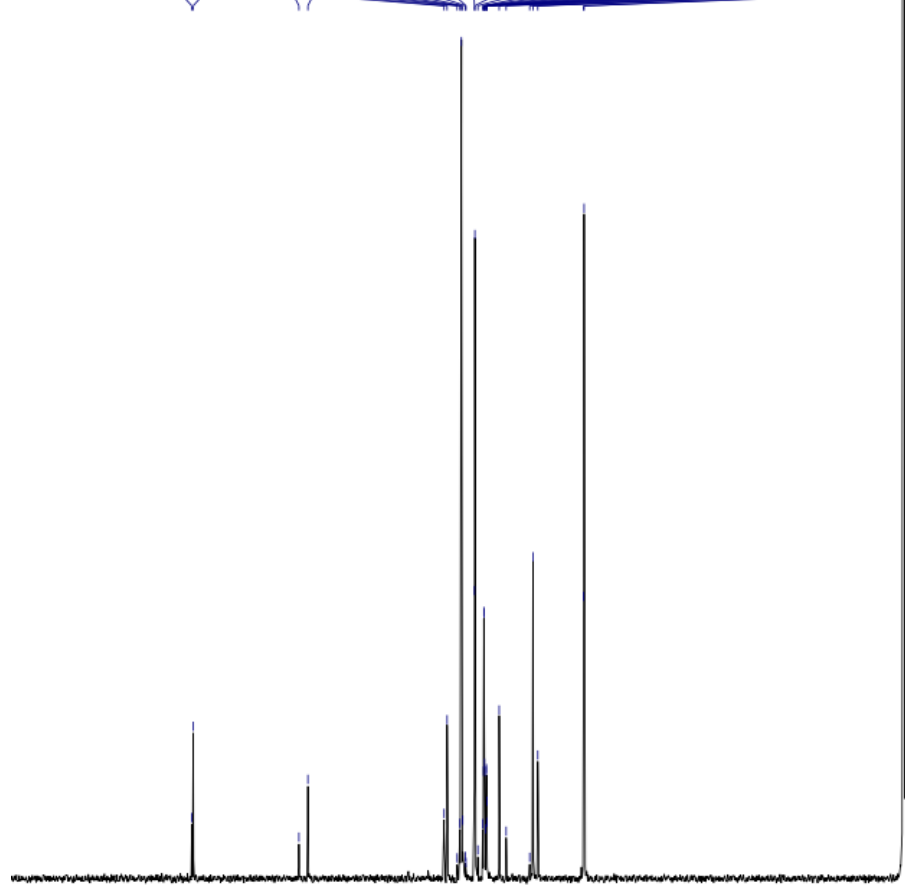

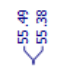
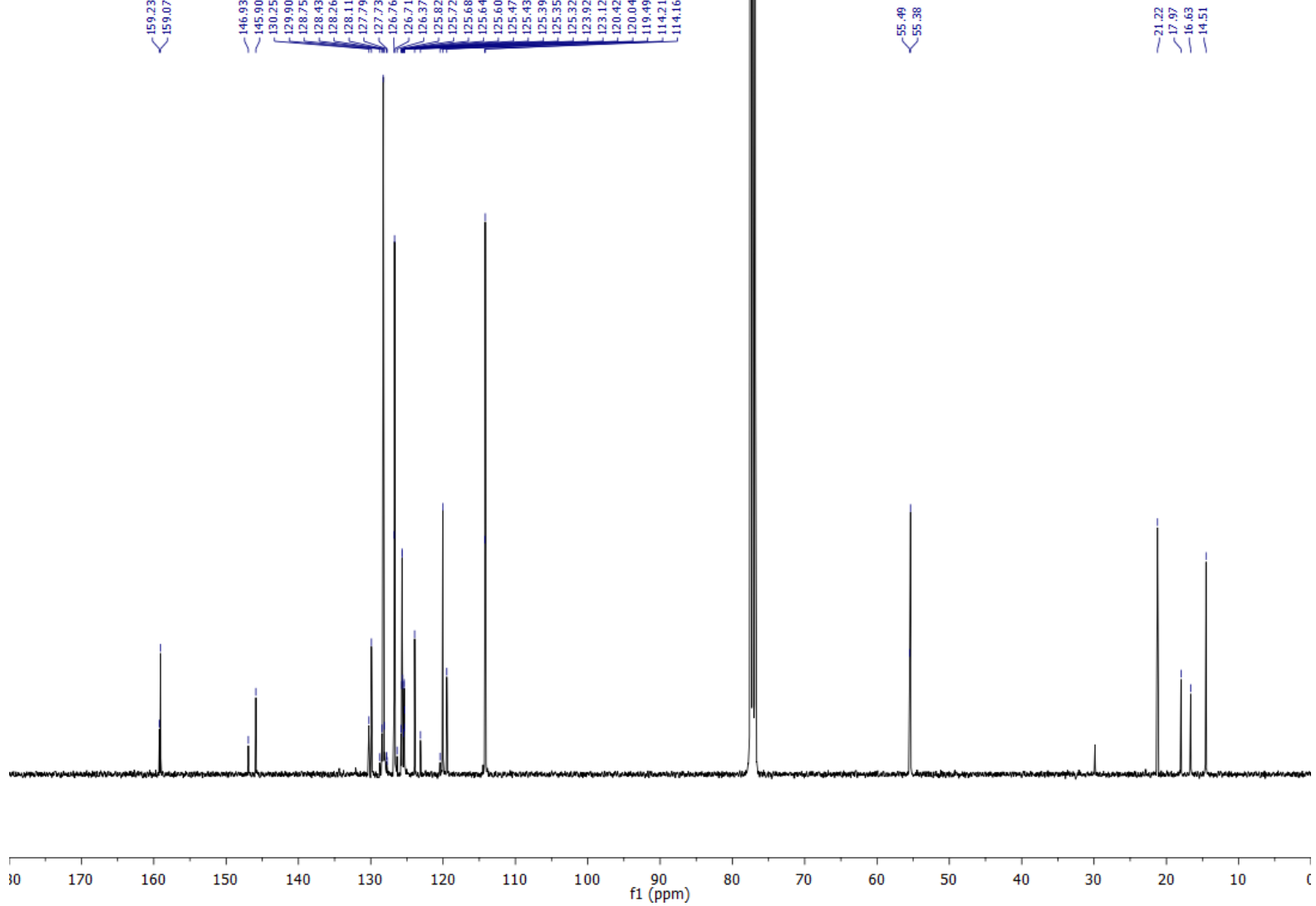
WILEY-VCH

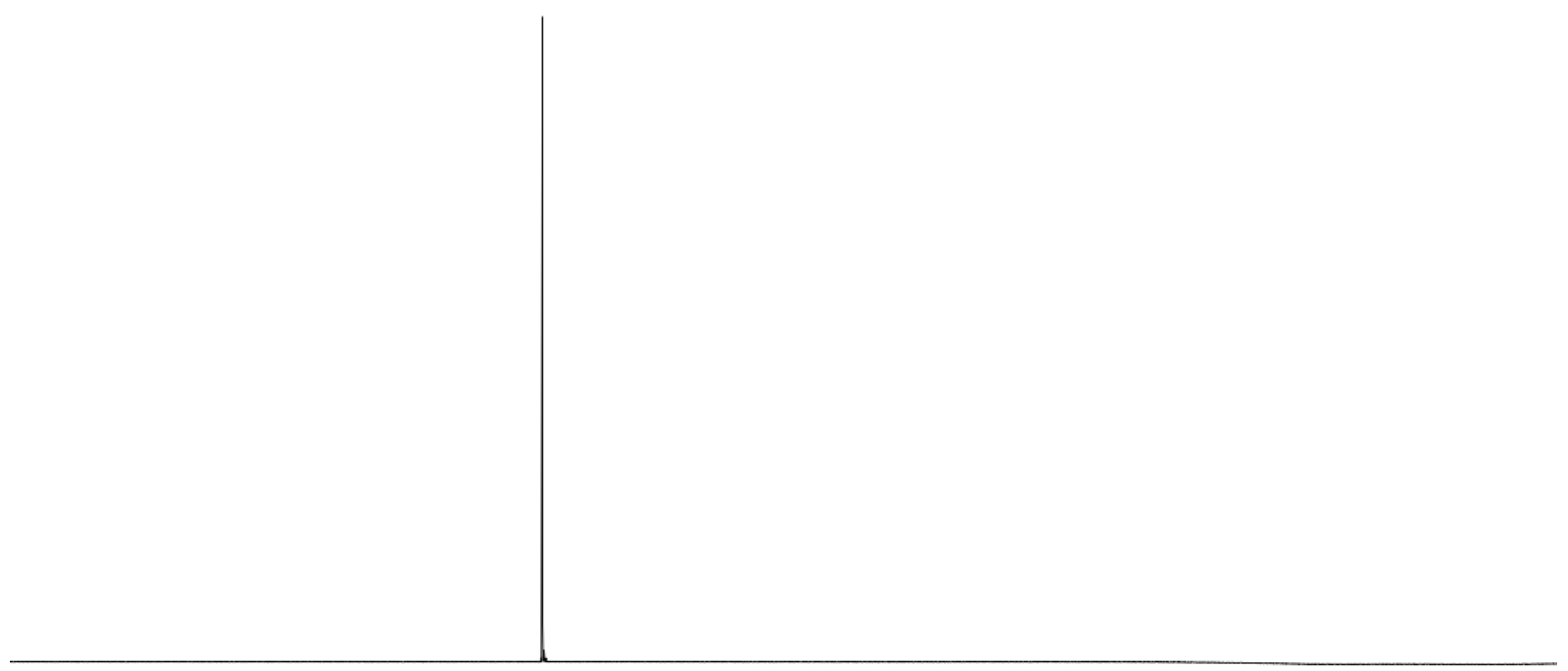

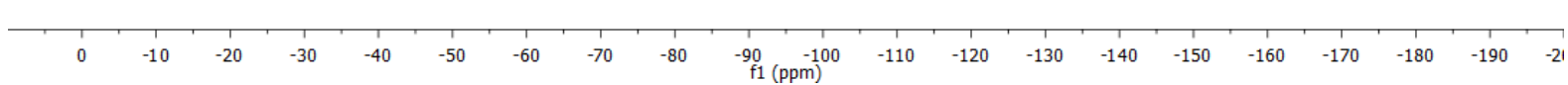




\section{(R)-1-(2-(4-methoxybenzylidene)cyclopropyl)-3-methylbenzene 6ah}

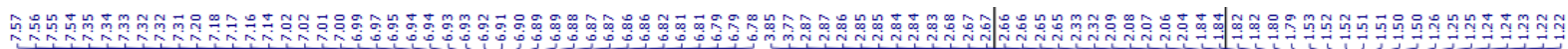
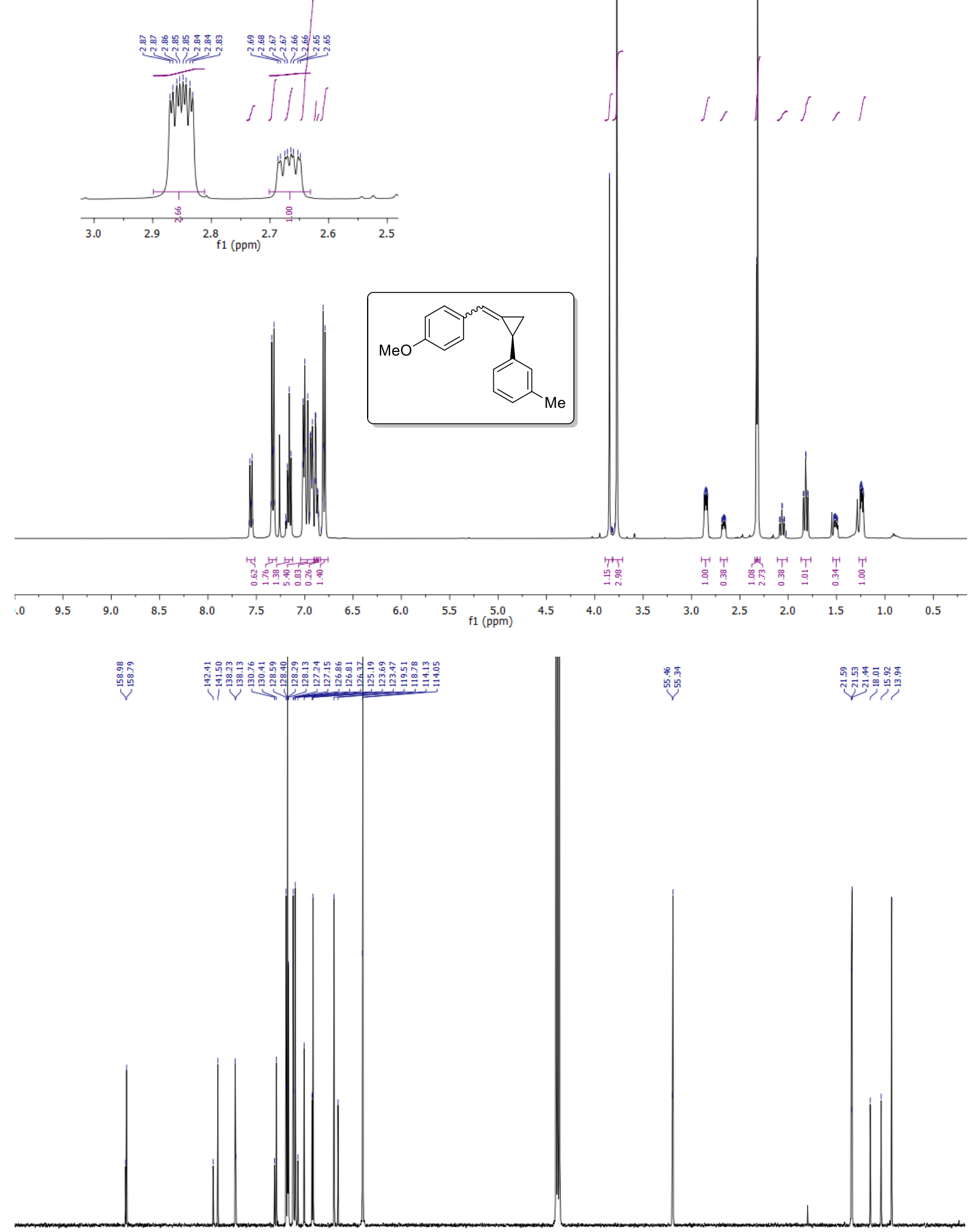

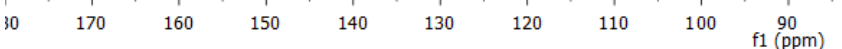

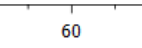

40 

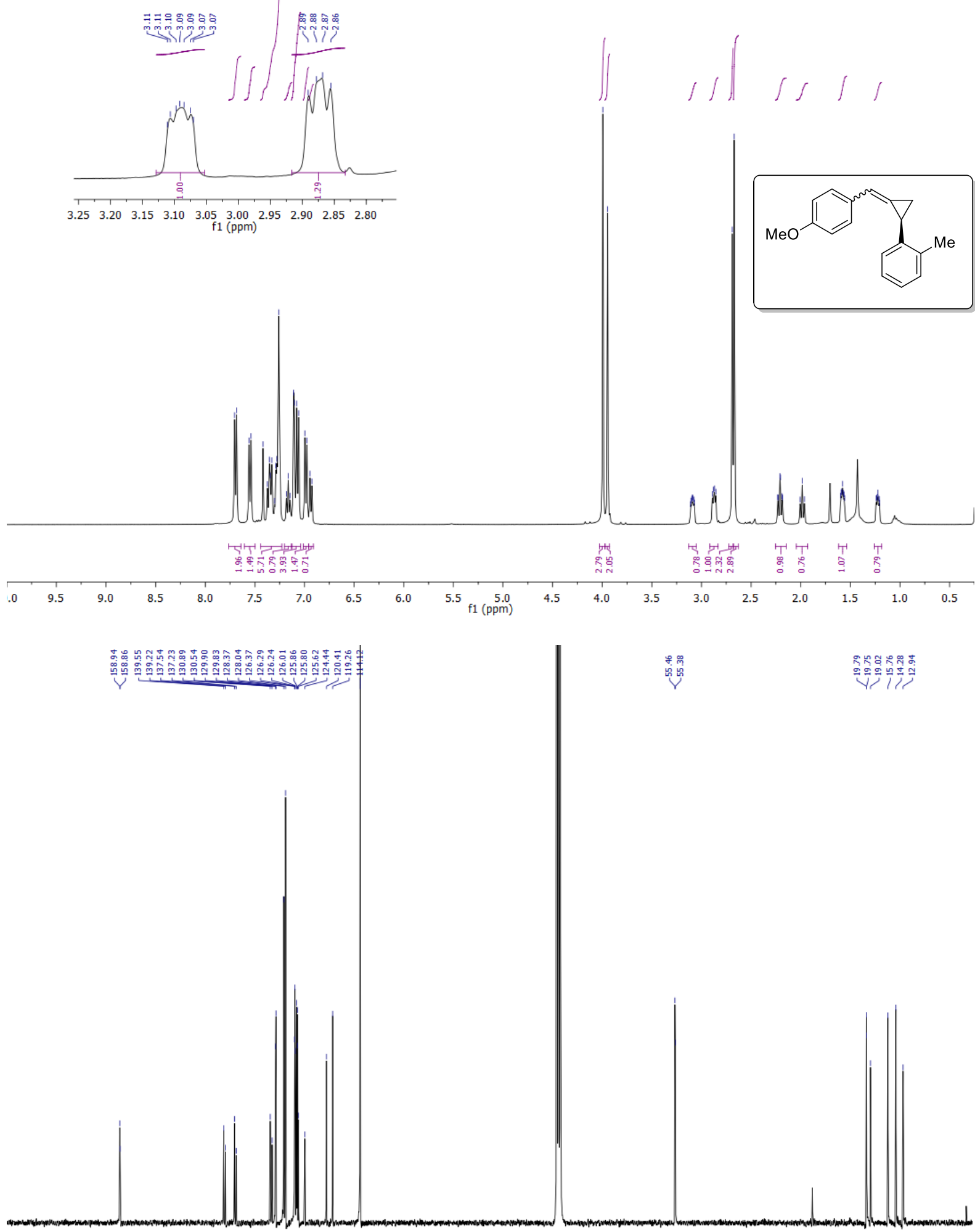

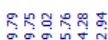




\section{(R)-3-(2-(4-methoxybenzylidene)cyclopropyl)thiophene 6aj}

mon
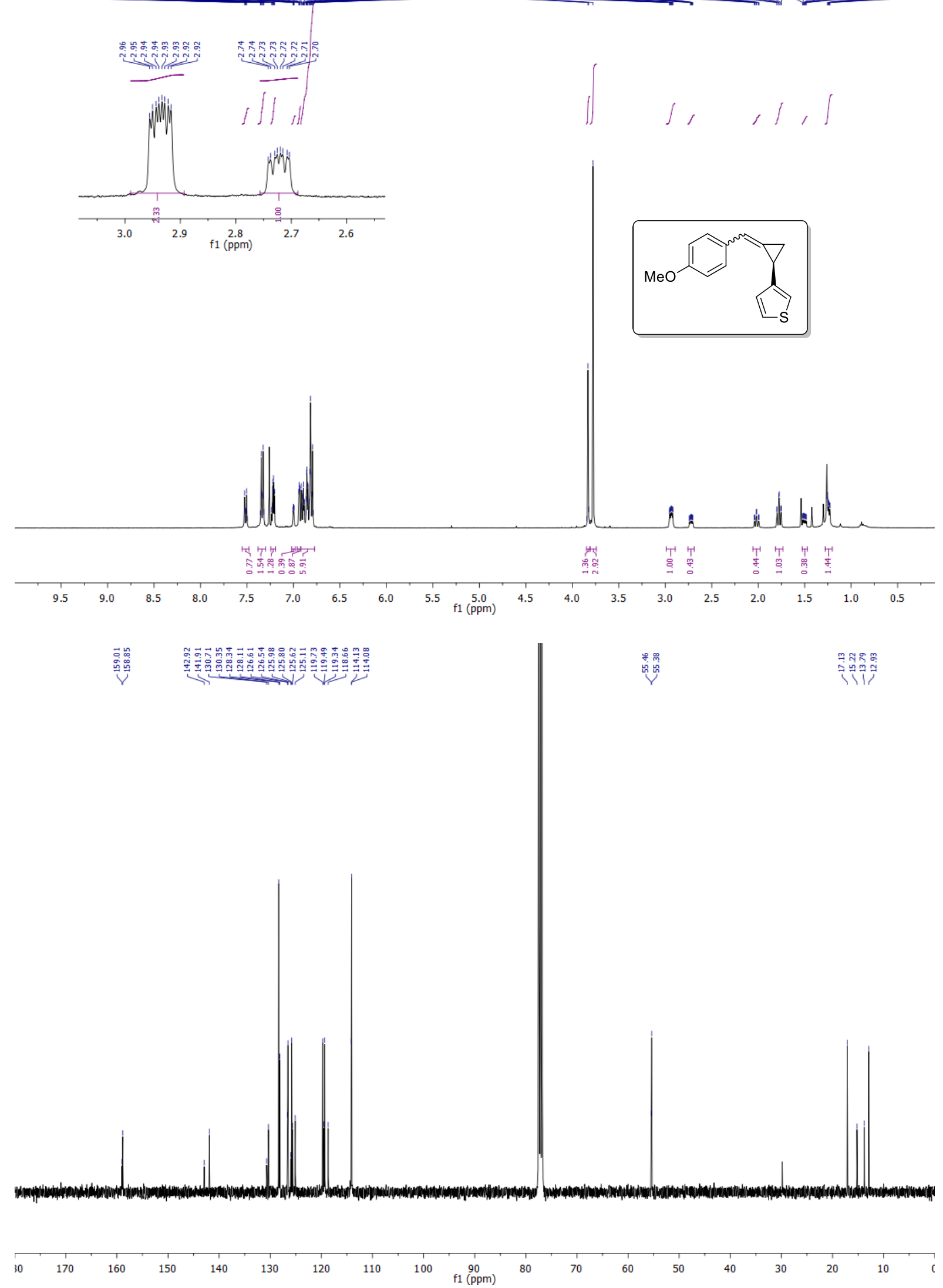


\section{(R)-3-(2-(4-methoxybenzylidene)cyclopropyl)-1-tosyl-1H-indole 6ak}
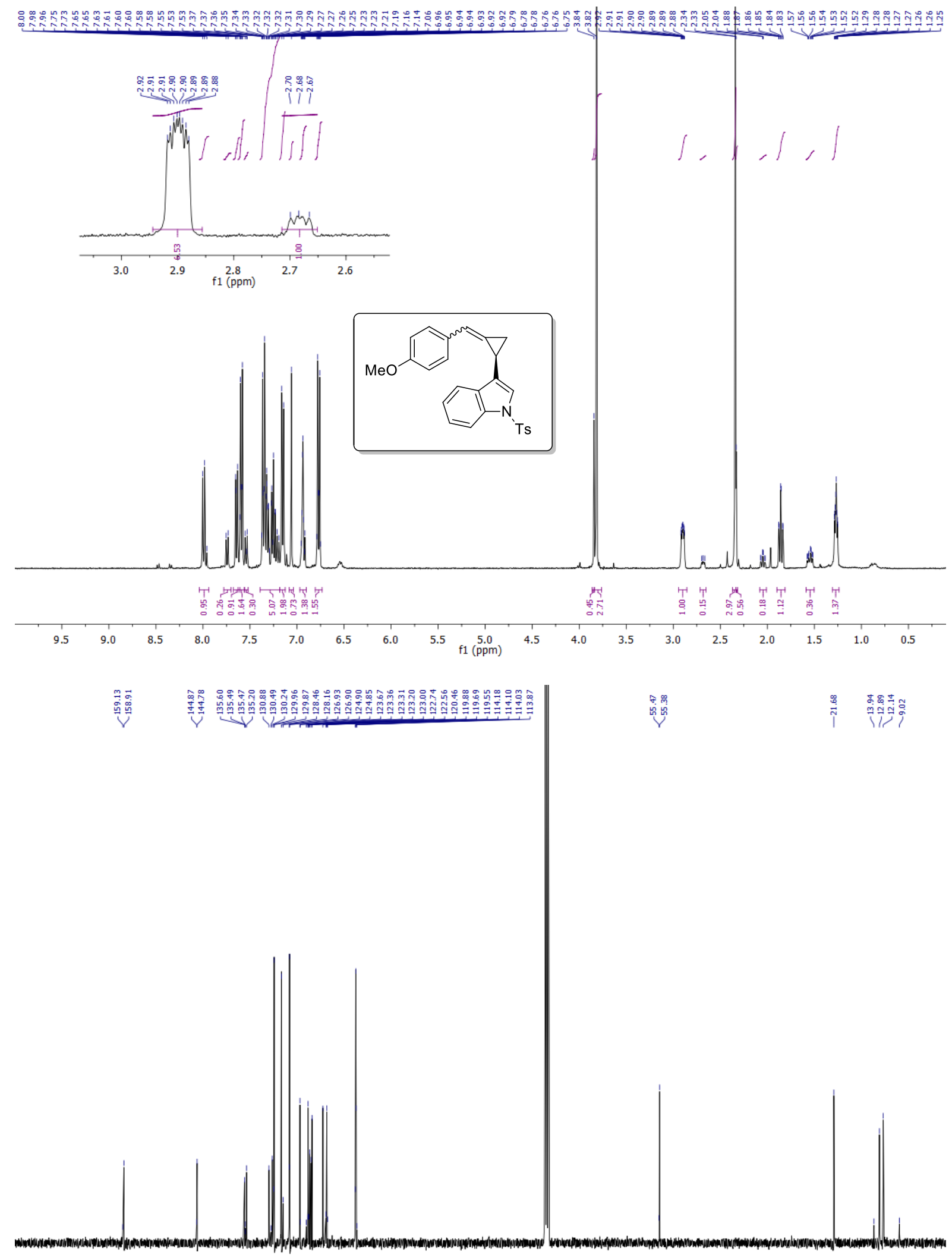

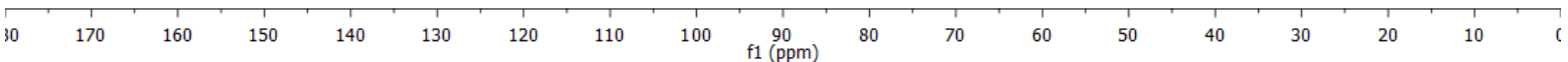




\section{(S)-1-((2-cyclohexylcyclopropylidene)methyl)-4-methoxybenzene 6al}

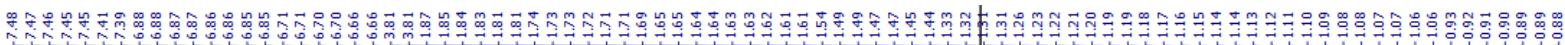
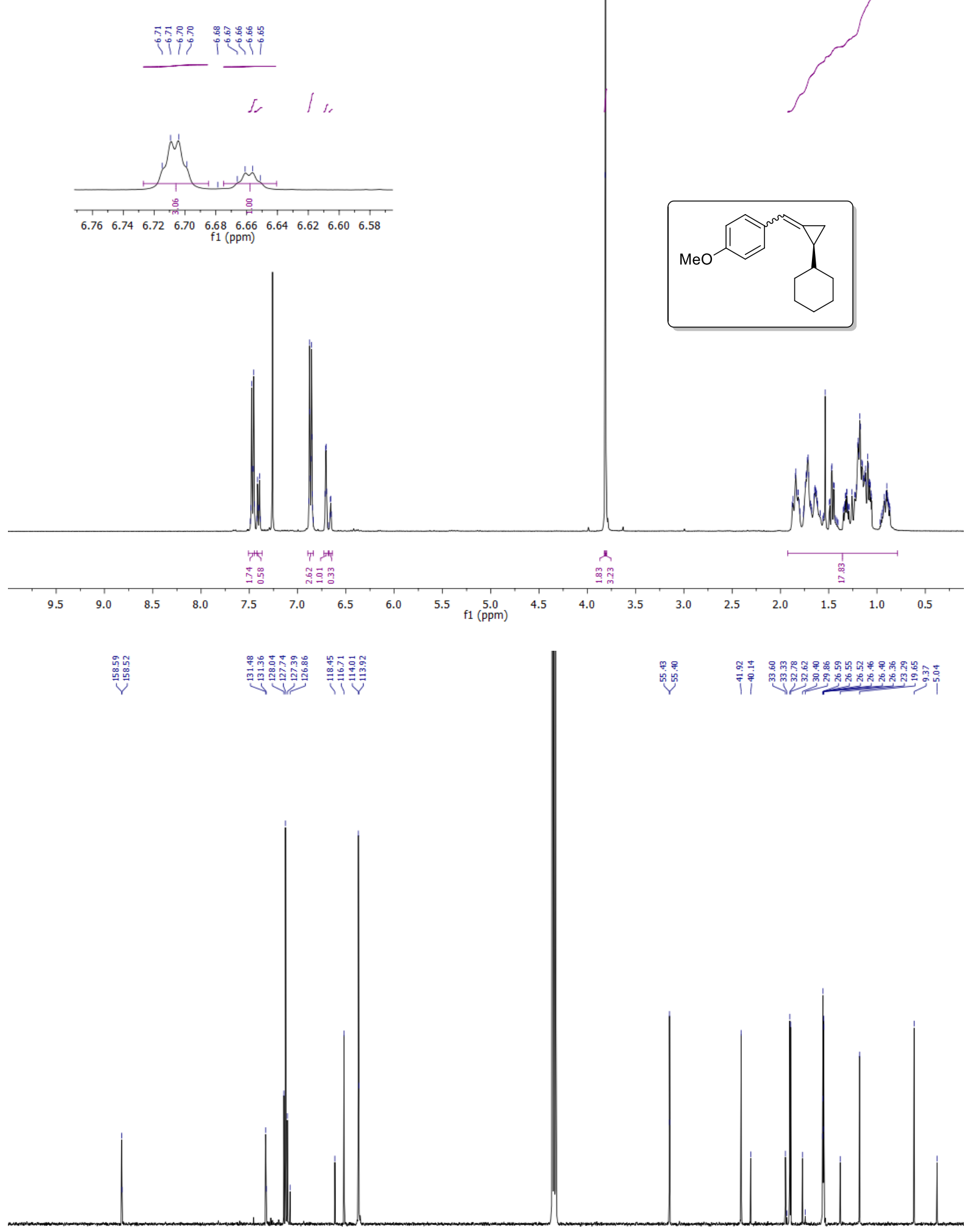

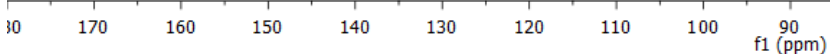

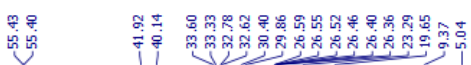

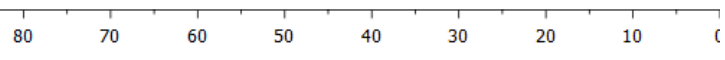




\section{$\underline{(R)-4-((2-p h e n y l c y c l o p r o p y l i d e n e) m e t h y l) p h e n o l ~} 15$}

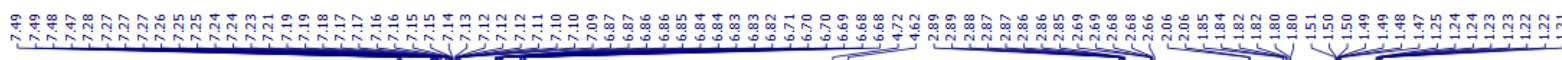
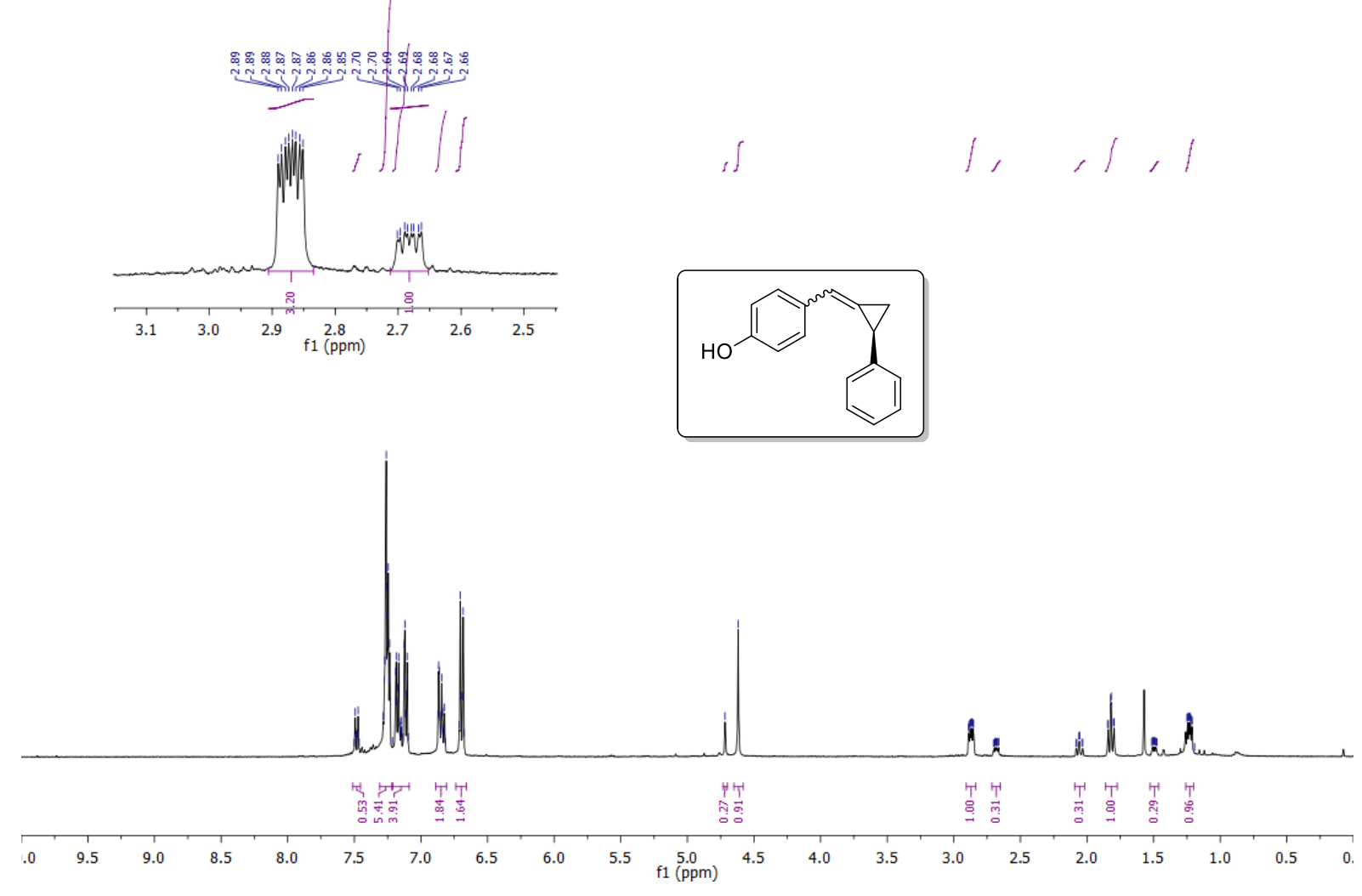

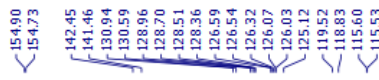

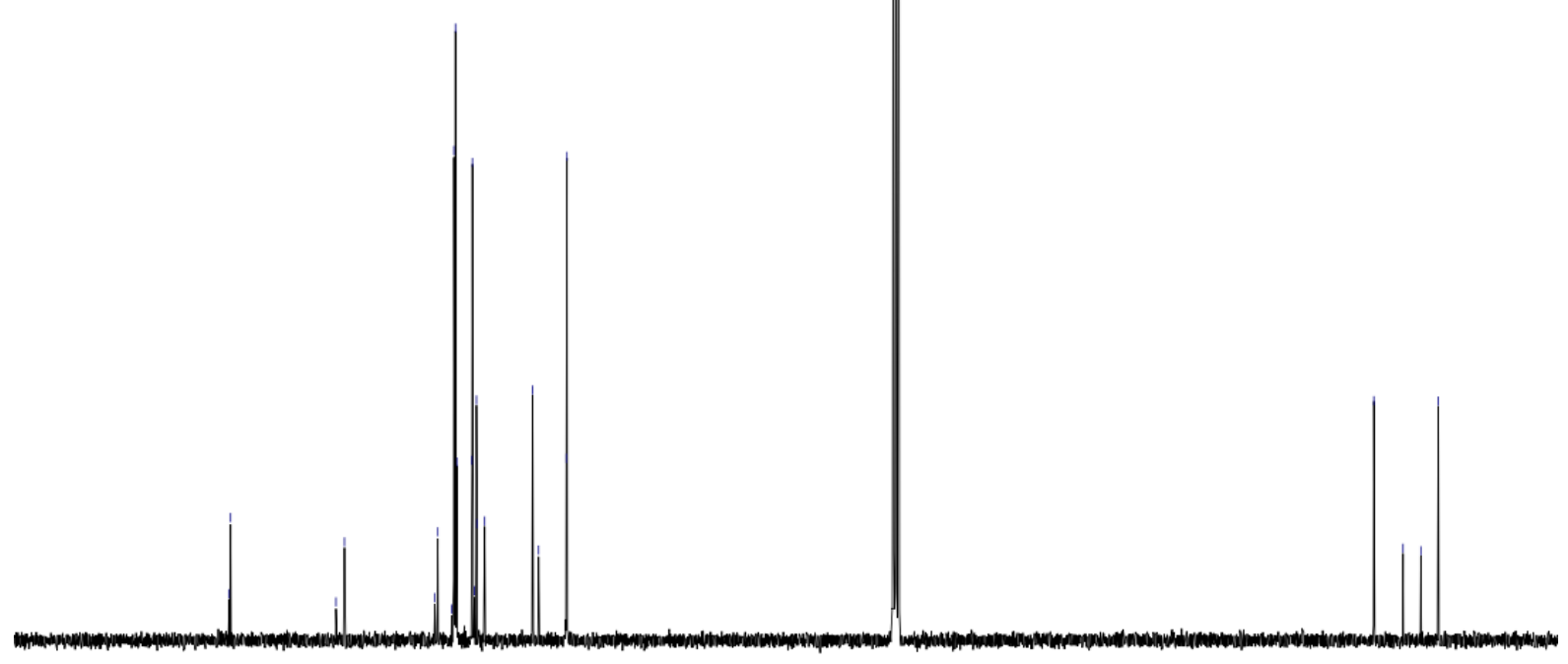

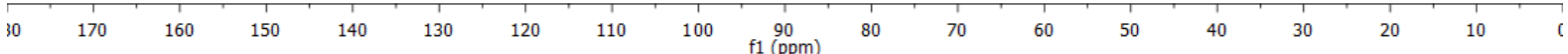



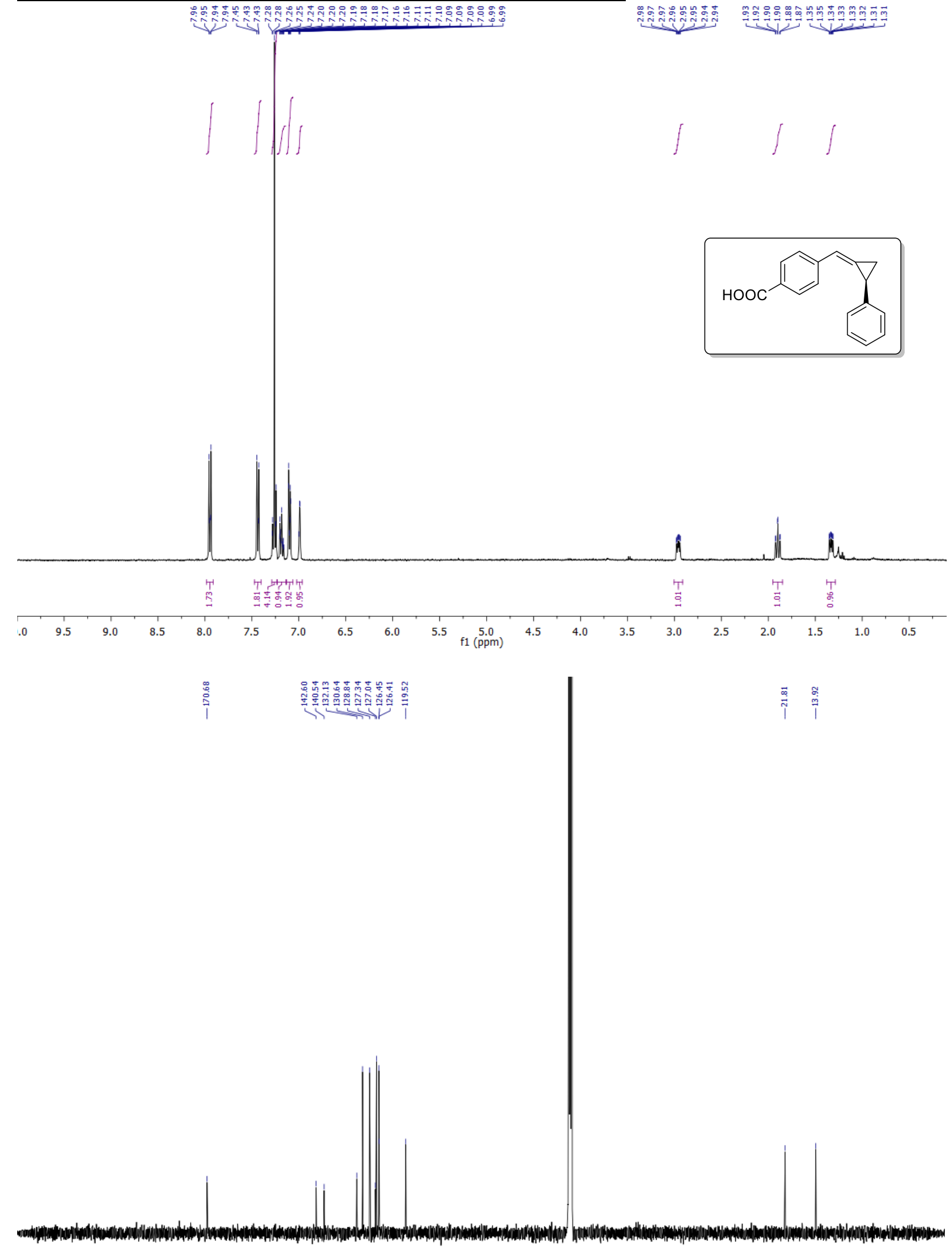

$\begin{array}{lllllllllllllllllllllll}210 & 200 & 190 & 180 & 170 & 160 & 150 & 140 & 130 & 120 & 110 & \underset{\mathrm{f} 1}{100}(\mathrm{ppm}) & 90 & 80 & 70 & 60 & 50 & 40 & 30 & 20 & 10 & 0 & -10\end{array}$ 
Methyl 4-(((1R,2R)-2-phenylcyclopropyl)methyl)benzoate 8

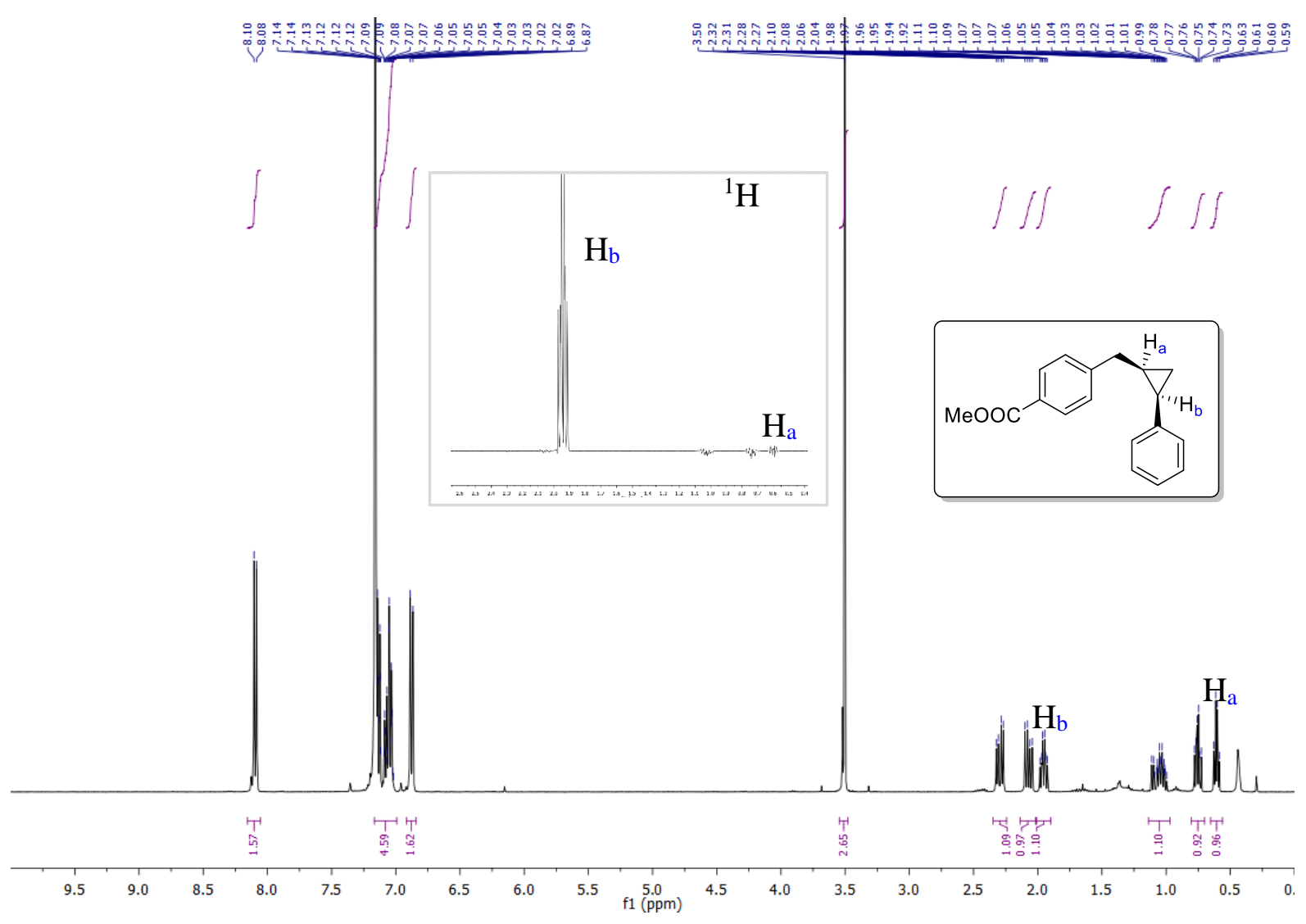

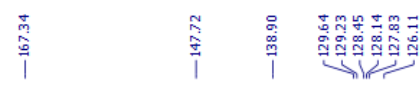

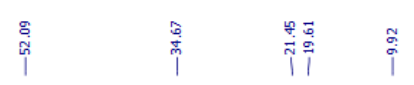




\section{Methyl (R)-4-(3-phenylbutyl)benzoate 10}

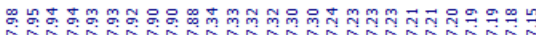

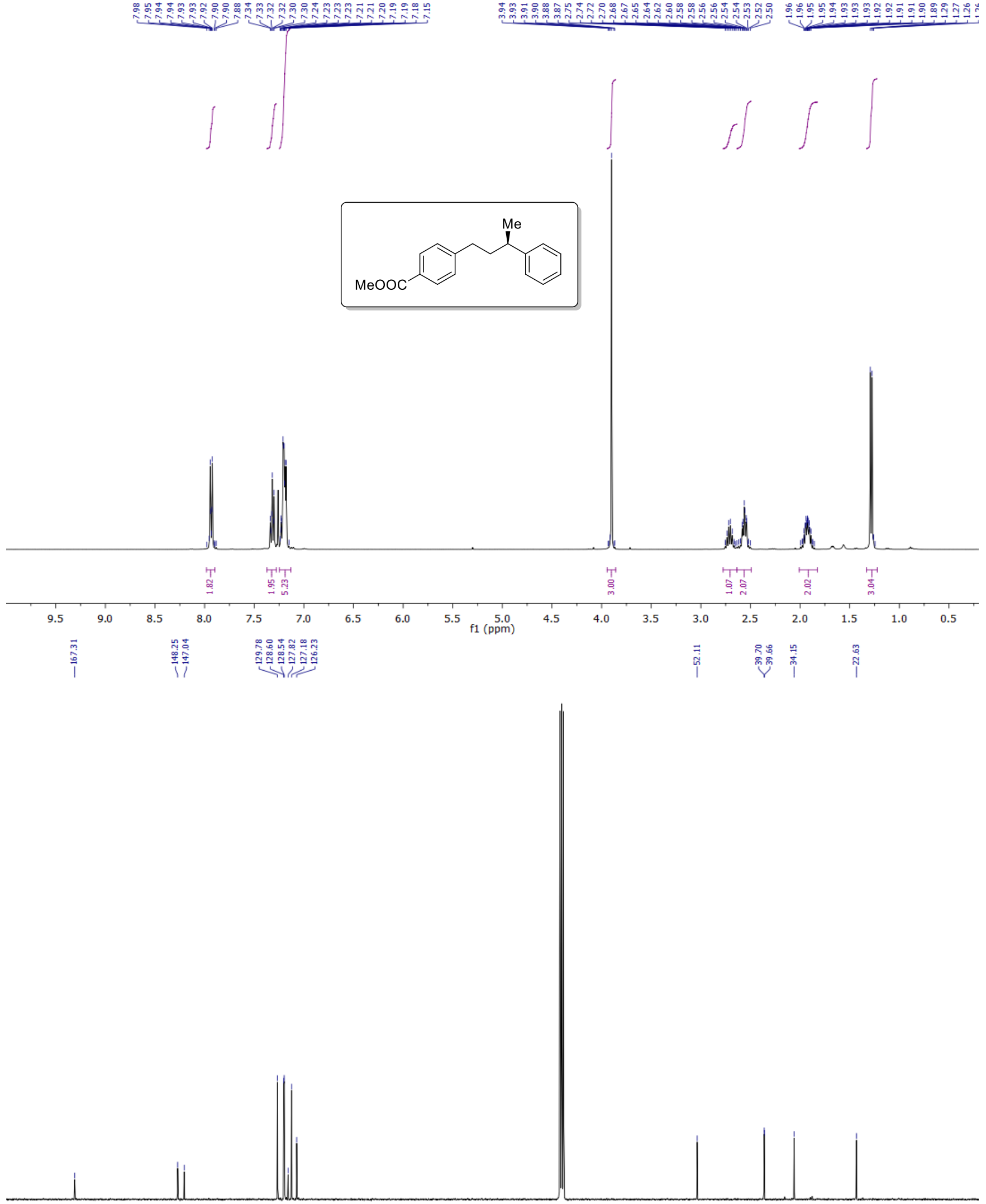

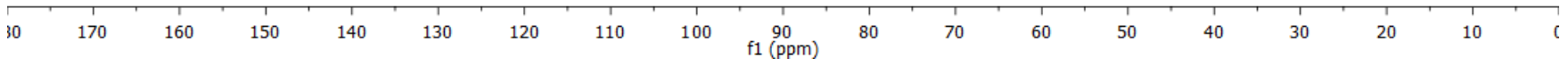




\section{References}

[1] P. G. Dormer, K. K. Eng, R. N. Farr, G. R. Humphrey, J. C. MaWilliams, P. J. Reider, J. W. Sager, R. P. Volante, J. Org. Chem. 2003, 68, 467.

[2] W. Ma, F. Chen, Y. Liu, Y.-M. He, Q.-H. Fan, Org. Lett. 2016, 18, 2730.

[3] J. Tan, T. Zheng, Y. Yu, K. Xu, RSC Adv. 2017, 7, 15176.

[4] R. Zong, D. Wang, R. Hammitt, R. P. Thummel, J. Org. Chem. 2006, 71, 167.

[5] J. Diesel, A. M. Finogenova, N. Cramer, J. Am. Chem. Soc. 2018, 140, 4489.

[6] E. Spahn, A. Albright, M. Shevlin, L. Pauli, A. Pfaltz, R. E. Gawley, J. Org. Chem. 2013, 78, 2731.

[7] Y. Cai, J.-W. Zhang, F. Li, J.-M. Liu, S.-L. Shi, ACS Catal. 2019, 9, 1.

[8] Prepared according to a literature procedure: J. Ren, Y. Liu, L. Song, R. Tong, Org. Lett. 2014, 16, 2986.

[9] S. Pal, Y.-Y. Zhou, C. Uyeda, J. Am. Chem. Soc. 2017, 139, 11686.

[10] Prepared according to a literature procedure: Y.-Y. Zhou, D. R. Hartline, T. J. Steiman, P. E. Fanwick, C. Uyeda, Inorg. Chem. 2014, 53, 11770.

[11]N. Suzuki, J. L. Hofstra, K. E. Poremba, S. E. Reisman, Org. Lett. 2017, 19, 2150. 